\title{
A Measurement of $B$ Meson Oscillations Using Inclusive Leptons in Proton-Antiproton Collisions at $1.8 \mathrm{TeV}$ \\ by \\ Marc David Peters
}

A.B. (Harvard University) 1990

M.A. (University of California, Berkeley) 1992

A dissertation submitted in partial satisfaction of the

requirements for the degree of

Doctor of Philosophy

in

Physics

in the

GRADUATE DIVISION

of the

UNIVERSITY OF CALIFORNIA, BERKELEY

Committee in charge:

Professor Marjorie D. Shapiro, Chair

Professor Kam-Biu Luk

Dean Joseph Cerny

Spring 1997 
The dissertation of Marc David Peters is approved:

\begin{tabular}{cc}
\hline Chair & Date \\
\hline & Date \\
\hline & Date
\end{tabular}

Date

University of California, Berkeley

Spring 1997 


\title{
A Measurement of $B$ Meson Oscillations Using Inclusive Leptons in Proton-Antiproton Collisions at $1.8 \mathrm{TeV}$
}

\author{
Copyright (C) 1997 \\ by \\ Marc David Peters
}

This work was supported by the Director, Office of Energy Research, Office of High Energy and Nuclear Physics, Division of High Energy Physics, of the U.S. Department of Energy under contract DE-AC03-76SF00098.

The United States Department of Energy has the right to use this thesis for any purpose whatsoever including the right to reproduce all or any part thereof. 


\author{
Abstract \\ A Measurement of $B$ Meson Oscillations Using Inclusive \\ Leptons in Proton-Antiproton Collisions at $1.8 \mathrm{TeV}$ \\ by \\ Marc David Peters \\ Doctor of Philosophy in Physics \\ University of California, Berkeley \\ Professor Marjorie D. Shapiro, Chair
}

The mass difference $\Delta m_{d}=M\left(B_{H}^{0}\right)-M\left(B_{L}^{0}\right)$ is measured from the time dependence of $B_{d}^{0}-\bar{B}_{d}^{0}$ oscillations, using data from the CDF experiment's inclusive electron and muon samples, collected during the 1994-1995 Fermilab Tevatron $p \bar{p}$ collider Run. Leptons are associated with a displaced vertex to select events from $b \bar{b}$ production. The proper time is reconstructed from the position, momentum, and invariant mass of the vertex. The final state of the $b$ hadron is determined from the charge of the trigger lepton; the initial state is determined by soft lepton and jet charge flavor-tagging. The combined fit of the electron and muon datasets using both flavor-tagging methods yields $\Delta m_{d}=0.47 \pm 0.06 \pm 0.04 \mathrm{ps}^{-1}$, where the first error is statistical and the second error is systematic. This corresponds to a value of $x_{d} \equiv \frac{\Delta m_{d}}{\Gamma_{B_{d}^{0}}}=0.73 \pm 0.10 \pm 0.07$, where $\Gamma_{B_{d}^{0}}=0.64 \pm 0.02 \mathrm{ps}^{-1}$. 
Dedicated to my wife, Erica Jessie Peters.

So let it be written; so let it be done. 


\section{Acknowledgements}

Over the course of my seven years as a graduate student, I have been helped by so many people it seems scarcely possible to thank them all.

First, thanks to everyone who made me feel welcome when I first arrived at Berkeley. Professors S. Davis and R. Ely made teaching laboratory electronics a lot of fun. Morning Tea at Strada was always the high point of the week, and I always appreciated seeing the regular cast of characters whenever I returned to town. Thanks for keeping the tradition going.

My first major responsibility as a member of CDF was overseeing the construction of the SVX' electronics, under the guiding hand of Carl Haber. (Carl also made the commute to Santa Clara almost fun.) Glenn, Phansu, Kim, Kimmie, and Jennifer at Promex did a fabulous job in ensuring that CDF had a world-class silicon detector. Thanks must also go to Joe I., Eric K., Dave S., and Nicola B. for teaching me about careful testing procedures.

Paul Derwent helped me enormously with the $\mathrm{SVX}^{\prime}$ radiation monitors. He patiently taught me everything about the system, and carried the pager for me far too many times, on far too short notice. Thanks also to the many Sci-Cos with whom I shared many aborts, usually at 3 am.

I'd like to thank everyone in the Lawrence Berkeley (National) Laboratory CDF group. There are many advantages to working for a government lab, not the least of which is having very bright and dedicated physicists for colleagues. Of the graduate students, William was an excellent role model and provided a lot of advice when it came time to produce this document. Bill helped maintain my sanity out on the 
dull, flat prairie. Next time we go to dinner at "the usual place," it's on me.

The CDF Bottom Group shaped this analysis in almost every respect. The rather time-consuming BPAD processing was made much less painful through the assistance of Slawek, Jonathan, Fumi, Wolfgang, Steve P., and Eric W. My work with $d E / d x$ would not have been possible without the help of Hovhannes, Barry W., Pat L., and Dave S. Special thanks go to Hans W., who identified a number of problems before anyone else even thought to look. I have been around long enough to have "served" under a number of conveners. I have learned much from their dedication to physics and to quality work. Fritz and Paris, in particular, were the source of many puzzling questions and illuminating insights.

Thanks also to my friends at Harvard: Dave K. paved the way for my work with soft leptons. The mixing group, Colin, George, Raimund, and Jorge, was the source of many productive and informative discussions. George, we will always miss you.

I would like to thank my dissertation committee, Joseph Cerny, Kam-Biu Luk, and Marjorie Shapiro. Their assistance improved this document an extraordinary amount. Any errors which remain are mine alone, though I am grateful that there are far fewer of them. More thanks must go to Marjorie for having been my advisor all these years. She, more than anyone else, taught me how to see the forest for the trees, and how to base one's self-respect on a job well-done.

This analysis would never have been completed without the hard work and dedication of my close colleagues, Marjorie, Manfred, Owen, and Joe. They graciously endured my idiosyncracies, and always gave me the best advice and assistance. Together, we have accomplished something of which we can be very proud. Thanks, gang - you're the best.

Finally, I can’t thank my family enough. My wife, Erica, has been very patient and understanding ever since I started writing. It won't be long before I can return the favor. And Mom, it may have taken a long time, but it's finally done. Thank you so much for your love and support. 


\section{The CDF Collaboration}

F. Abe, ${ }^{17}$ H. Akimoto, ${ }^{36}$ A. Akopian, ${ }^{31}$ M. G. Albrow, ${ }^{7}$ S. R. Amendolia,,${ }^{27}$ D. Amidei,${ }^{20}$ J. Antos,${ }^{33}$ S. Aota,${ }^{36}$ G. Apollinari,${ }^{31}$ T. Asakawa,${ }^{36}$ W. Ashmanskas,${ }^{18}$ M. Atac ${ }^{7}$ F. Azfar, ${ }^{26}$ P. Azzi-Bacchetta,${ }^{25}$ N. Bacchetta, ${ }^{25}$ W. Badgett,${ }^{20}$ S. Bagdasarov ${ }^{31}$ M. W. Bailey,${ }^{22}$ J. Bao,${ }^{39}$ P. de Barbaro, ${ }^{30}$ A. Barbaro-Galtieri, ${ }^{18}$ V. E. Barnes, ${ }^{29}$ B. A. Barnett,${ }^{15}$ M. Barone,${ }^{9}$ E. Barzi,${ }^{9}$ G. Bauer,${ }^{19}$ T. Baumann,${ }^{11}$ F. Bedeschi, ${ }^{27}$ S. Behrends, ${ }^{3}$ S. Belforte, ${ }^{27}$ G. Bellettini, ${ }^{27}$ J. Bellinger, ${ }^{38}$ D. Benjamin, ${ }^{35}$ J. Benlloch, ${ }^{19}$ J. Bensinger,${ }^{3}$ D. Benton, ${ }^{26}$ A. Beretvas, ${ }^{7}$ J. P. Berge, ${ }^{7}$ J. Berryhill, ${ }^{5}$ S. Bertolucci, ${ }^{9}$ B. Bevensee, ${ }^{26}$ A. Bhatti,${ }^{31}$ K. Biery, ${ }^{7}$ M. Binkley, ${ }^{7}$ D. Bisello, ${ }^{25}$ R. E. Blair, ${ }^{1}$ C. Blocker,${ }^{3}$ A. Bodek,${ }^{30}$ W. Bokhari, ${ }^{19}$ V. Bolognesi, ${ }^{2}$ G. Bolla ${ }^{29}$ D. Bortoletto,${ }^{29}$ J. Boudreau, ${ }^{28}$ L. Breccia, ${ }^{2}$ C. Bromberg, ${ }^{21}$ N. Bruner,${ }^{22}$ E. Buckley-Geer, ${ }^{7}$ H. S. Budd,${ }^{30}$ K. Burkett,${ }^{20}$ G. Busetto, ${ }^{25}$ A. Byon-Wagner, ${ }^{7}$ K. L. Byrum, ${ }^{1}$ J. Cammerata, ${ }^{15}$ C. Campagnari, ${ }^{7}$ M. Campbell, ${ }^{20}$ A. Caner, ${ }^{27}$ W. Carithers, ${ }^{18}$ D. Carlsmith, ${ }^{38}$ A. Castro, ${ }^{25}$ D. Cauz,${ }^{27}$ Y. Cen,${ }^{30}$ F. Cervelli, ${ }^{27}$ P. S. Chang, ${ }^{33}$ P. T. Chang, ${ }^{33}$ H. Y. Chao, ${ }^{33}$ J. Chapman, ${ }^{20}$ M. -T. Cheng, ${ }^{33}$ G. Chiarelli, ${ }^{27}$ T. Chikamatsu, ${ }^{36}$ C. N. Chiou, ${ }^{33}$ L. Christofek, ${ }^{13}$ S. Cihangir, ${ }^{7}$ A. G. Clark, ${ }^{10}$ M. Cobal, ${ }^{27}$ E. Cocca,${ }^{27}$ M. Contreras, ${ }^{5}$ J. Conway, ${ }^{32}$ J. Cooper, ${ }^{7}$ M. Cordelli, ${ }^{9}$ C. Couyoumtzelis, ${ }^{10}$ D. Crane,${ }^{1}$ D. Cronin-Hennessy,${ }^{6}$ R. Culbertson, ${ }^{5}$ T. Daniels, ${ }^{19}$ F. DeJongh ${ }^{7}$ S. Delchamps, ${ }^{7}$ S. Dell'Agnello, ${ }^{27}$ M. Dell'Orso, ${ }^{27}$ R. Demina,${ }^{7}$ L. Demortier, ${ }^{31}$ M. Deninno, ${ }^{2}$ P. F. Derwent,${ }^{7}$ T. Devlin,${ }^{32}$ J. R. Dittmann, ${ }^{6}$ S. Donati ${ }^{27}$ J. Done ${ }^{34}$ T. Dorigo, ${ }^{25}$ A. Dunn, ${ }^{20}$ N. Eddy,${ }^{20}$ K. Einsweiler, ${ }^{18}$ J. E. Elias, ${ }^{7}$ R. Ely,$^{18}$ E. Engels, Jr. ${ }^{28}$ D. Errede, ${ }^{13}$ S. Errede,${ }^{13}$ Q. Fan,${ }^{30}$ G. Feild, ${ }^{39}$ C. Ferretti, ${ }^{27}$ I. Fiori, ${ }^{2}$ B. Flaugher ${ }^{7}$ G. W. Foster,${ }^{7}$ M. Franklin, ${ }^{11}$ M. Frautschi ${ }^{35}$ J. Freeman, ${ }^{7}$ J. Friedman,${ }^{19}$ H. Frisch,${ }^{5}$ Y. Fukui,${ }^{17}$ S. Funaki, ${ }^{36}$ S. Galeotti, ${ }^{27}$ M. Gallinaro, ${ }^{26}$ O. Ganel, ${ }^{35}$ M. Garcia-Sciveres,${ }^{18}$ A. F. Garfinkel, ${ }^{29}$ C. Gay,${ }^{11}$ S. Geer, ${ }^{7}$ D. W. Gerdes,${ }^{15}$ P. Giannetti,${ }^{27}$ N. Giokaris,${ }^{31}$ P. Giromini ${ }^{9}$ G. Giusti ${ }^{27}$ L. Gladney, ${ }^{26}$ D. Glenzinski, ${ }^{15}$ M. Gold, ${ }^{22}$ J. Gonzalez,${ }^{26}$ A. Gordon, ${ }^{11}$ A. T. Goshaw ${ }^{6}$ Y. Gotra,${ }^{25}$ K. Goulianos, ${ }^{31}$ H. Grassmann,${ }^{27}$ L. Groer,${ }^{32}$ C. Grosso-Pilcher,${ }^{5}$ G. Guillian, ${ }^{20}$ R. S. Guo, ${ }^{33}$ C. Haber,${ }^{18}$ E. Hafen,${ }^{19}$ S. R. Hahn,${ }^{7}$ R. Hamilton, ${ }^{11}$ R. Handler,${ }^{38}$ R. M. Hans,${ }^{39}$ F. Happacher, ${ }^{9}$ K. Hara,${ }^{36}$ A. D. Hardman, ${ }^{29}$ B. Harral, ${ }^{26}$ R. M. Harris,${ }^{7}$ S. A. Hauger, ${ }^{6}$ J. Hauser, ${ }^{4}$ C. Hawk ${ }^{32}$ E. Hayashi, ${ }^{36}$ J. Heinrich ${ }^{26}$ B. Hinrichsen, ${ }^{14}$ K. D. Hoffman,${ }^{29}$ M. Hohlmann, ${ }^{5}$ C. Holck, ${ }^{26}$ R. Hollebeek, ${ }^{26}$ L. Holloway, ${ }^{13}$ S. Hong,${ }^{26}$ G. Houk,${ }^{26}$ P. $\mathrm{Hu}^{28}{ }^{28}$ B. T. Huffman, ${ }^{28}$ R. Hughes,${ }^{23}$ J. Huston, ${ }^{21}$ J. Huth, ${ }^{11}$ J. Hylen, ${ }^{7}$ H. Ikeda, ${ }^{36}$ M. Incagli, ${ }^{27} \mathrm{~J}$. Incandela,${ }^{7}$ G. Introzzi, ${ }^{27} \mathrm{~J}$. Iwai,${ }^{36} \mathrm{Y}$. Iwata,${ }^{12}$ H. Jensen,${ }^{7}$ U. Joshi,${ }^{7}$ R. W. Kadel,${ }^{18}$ E. Kajfasz ${ }^{25}$ H. Kambara, ${ }^{10}$ T. Kamon,${ }^{34}$ T. Kaneko, ${ }^{36}$ K. Karr ${ }^{37}$ H. Kasha ${ }^{39}$ Y. Kato, ${ }^{24}$ T. A. Keaffaber, ${ }^{29}$ K. Kelley, ${ }^{19}$ R. D. Kennedy, ${ }^{7}$ R. Kephart,${ }^{7}$ P. Kesten, ${ }^{18}$ D. Kestenbaum, ${ }^{11}$ H. Keutelian, ${ }^{7}$ F. Keyvan, ${ }^{4}$ B. Kharadia, ${ }^{13}$ B. J. Kim,${ }^{30}$ D. H. Kim,${ }^{7 a}$ H. S. Kim,${ }^{14}$ S. B. Kim,${ }^{20}$ S. H. Kim ${ }^{36}$ Y. K. Kim, ${ }^{18}$ L. Kirsch,${ }^{3}$ P. Koehn, ${ }^{23}$ K. Kondo, ${ }^{36}$ J. Konigsberg, ${ }^{8}$ S. Kopp,${ }^{5}$ K. Kordas,${ }^{14}$ A. Korytov,${ }^{8}$ W. Koska, ${ }^{7}$ E. Kovacs, ${ }^{7 a}$ W. Kowald, ${ }^{6}$ 
M. Krasberg, ${ }^{20}$ J. Kroll, ${ }^{7}$ M. Kruse,${ }^{30}$ T. Kuwabara,${ }^{36}$ S. E. Kuhlmann, ${ }^{1}$ E. Kuns,${ }^{32}$ A. T. Laasanen ${ }^{29}$ S. Lami,${ }^{27}$ S. Lammel,${ }^{7}$ J. I. Lamoureux,${ }^{3}$ M. Lancaster, ${ }^{18}$ T. LeCompte, ${ }^{1}$ S. Leone ${ }^{27}$ J. D. Lewis,${ }^{7}$ P. Limon,${ }^{7}$ M. Lindgren,${ }^{4}$ T. M. Liss,${ }^{13}$ J. B. Liu,${ }^{30}$ Y. C. Liu ${ }^{33}$ N. Lockyer, ${ }^{26}$ O. Long, ${ }^{26}$ C. Loomis, ${ }^{32}$ M. Loreti, ${ }^{25}$ J. Lu ${ }^{34}$ D. Lucchesi ${ }^{27}$ P. Lukens, ${ }^{7}$ S. Lusin,${ }^{38}$ J. Lys,${ }^{18}$ K. Maeshima, ${ }^{7}$ A. Maghakian,${ }^{31}$ P. Maksimovic, ${ }^{19}$ M. Mangano, ${ }^{27}$ J. Mansour, ${ }^{21}$ M. Mariotti, ${ }^{25}$ J. P. Marriner, ${ }^{7}$ A. Martin ${ }^{39}$ J. A. J. Matthews, ${ }^{22}$ R. Mattingly, ${ }^{19}$ P. McIntyre, ${ }^{34}$ P. Melese, ${ }^{31}$ A. Menzione ${ }^{27}$ E. Meschi ${ }^{27}$ S. Metzler,${ }^{26}$ C. Miao, ${ }^{20}$ T. Miao,${ }^{7}$ G. Michail,${ }^{11}$ R. Miller, ${ }^{21}$ H. Minato, ${ }^{36}$ S. Miscetti,${ }^{9}$ M. Mishina,${ }^{17}$ H. Mitsushio, ${ }^{36}$ T. Miyamoto,${ }^{36}$ S. Miyashita,${ }^{36}$ N. Moggi, ${ }^{27}$ Y. Morita,${ }^{17}$ A. Mukherjee,${ }^{7}$ T. Muller, ${ }^{16}$ P. Murat,${ }^{27}$ H. Nakada, ${ }^{36}$ I. Nakano, ${ }^{36}$ C. Nelson, ${ }^{7}$ D. Neuberger, ${ }^{16}$ C. Newman-Holmes, ${ }^{7}$ C-Y. P. Ngan,${ }^{19}$ M. Ninomiya, ${ }^{36}$ L. Nodulman, ${ }^{1}$ S. H. Oh, ${ }^{6}$ K. E. Ohl,${ }^{39}$ T. Ohmoto, ${ }^{12}$ T. Ohsugi, ${ }^{12}$ R. Oishi,${ }^{36}$ M. Okabe, ${ }^{36}$ T. Okusawa,${ }^{24}$ R. Oliveira,${ }^{26}$ J. Olsen,${ }^{38}$ C. Pagliarone, ${ }^{27}$ R. Paoletti, ${ }^{27}$ V. Papadimitriou, ${ }^{35}$ S. P. Pappas,${ }^{39}$ N. Parashar, ${ }^{27}$ S. Park ${ }^{7}$ A. Parri ${ }^{9}$ J. Patrick, ${ }^{7}$ G. Pauletta, ${ }^{27}$ M. Paulini,${ }^{18}$ A. Perazzo, ${ }^{27}$ L. Pescara, ${ }^{25}$ M. D. Peters, ${ }^{18}$ T. J. Phillips,${ }^{6}$ G. Piacentino, ${ }^{27}$ M. Pillai,${ }^{30}$ K. T. Pitts ${ }^{7}$ R. Plunkett,${ }^{7}$ L. Pondrom ${ }^{38}$ J. Proudfoot,${ }^{1}$ F. Ptohos,${ }^{11}$ G. Punzi, ${ }^{27}$ K. Ragan,${ }^{14}$ D. Reher, ${ }^{18}$ A. Ribon,${ }^{25}$ F. Rimondi,${ }^{2}$ L. Ristori,${ }^{27}$ W. J. Robertson, ${ }^{6}$ T. Rodrigo,${ }^{27}$ S. Rolli, ${ }^{37}$ J. Romano, ${ }^{5}$ L. Rosenson, ${ }^{19}$ R. Roser,${ }^{13}$ T. Saab, ${ }^{14}$ W. K. Sakumoto, ${ }^{30}$ D. Saltzberg, ${ }^{5}$ A. Sansoni, ${ }^{9}$ L. Santi, ${ }^{27}$ H. Sato,${ }^{36}$ P. Schlabach, ${ }^{7}$ E. E. Schmidt,${ }^{7}$ M. P. Schmidt, ${ }^{39}$ A. Scribano, ${ }^{27}$ S. Segler ${ }^{7}$ S. Seidel, ${ }^{22}$ Y. Seiya,${ }^{36}$ G. Sganos,${ }^{14}$ M. D. Shapiro, ${ }^{18}$ N. M. Shaw,${ }^{29}$ Q. Shen,${ }^{29}$ P. F. Shepard, ${ }^{28}$ M. Shimojima,${ }^{36}$ M. Shochet, ${ }^{5}$ J. Siegrist,${ }^{18}$ A. Sill, ${ }^{35}$ P. Sinervo, ${ }^{14}$ P. Singh, ${ }^{28}$ J. Skarha,,${ }^{15}$ K. Sliwa,${ }^{37}$ F. D. Snider,${ }^{15}$ T. Song, ${ }^{20}$ J. Spalding, ${ }^{7}$ T. Speer, ${ }^{10}$ P. Sphicas,${ }^{19}$ F. Spinella, ${ }^{27}$ M. Spiropulu,${ }^{11}$ L. Spiegel,${ }^{7}$ L. Stanco,${ }^{25}$ J. Steele, ${ }^{38}$ A. Stefanini, ${ }^{27}$ K. Strahl, ${ }^{14}$ J. Strait, ${ }^{7}$ R. Ströhmer,${ }^{7 a}$ D. Stuart, ${ }^{7}$ G. Sullivan,${ }^{5}$ K. Sumorok,${ }^{19}$ J. Suzuki ${ }^{36}$ T. Takada ${ }^{36}$ T. Takahashi, ${ }^{24}$ T. Takano,${ }^{36}$ K. Takikawa ${ }^{36}$ N. Tamura, ${ }^{12}$ B. Tannenbaum, ${ }^{22}$ F. Tartarelli,${ }^{27}$ W. Taylor,${ }^{14}$ P. K. Teng ${ }^{33}$ Y. Teramoto, ${ }^{24}$ S. Tether,${ }^{19}$ D. Theriot,${ }^{7}$ T. L. Thomas,${ }^{22}$ R. Thun,${ }^{20}$ R. Thurman-Keup, ${ }^{1}$ M. Timko, ${ }^{37}$ P. Tipton, ${ }^{30}$ A. Titov,${ }^{31}$ S. Tkaczyk,${ }^{7}$ D. Toback,${ }^{5}$ K. Tollefson,${ }^{30}$ A. Tollestrup ${ }^{7}$ H. Toyoda,${ }^{24}$ W. Trischuk,${ }^{14}$ J. F. de Troconiz,,${ }^{11}$ S. Truitt, ${ }^{20}$ J. Tseng, ${ }^{19}$ N. Turini,${ }^{27}$ T. Uchida,${ }^{36}$ N. Uemura,${ }^{36}$ F. Ukegawa,,${ }^{26}$ G. Unal, ${ }^{26}$ J. Valls ${ }^{7 a}$ S. C. van den Brink,${ }^{28}$ S. Vejcik, III, ${ }^{20}$ G. Velev, ${ }^{27}$ R. Vidal, ${ }^{7}$ R. Vilar, ${ }^{7 a}$ M. Vondracek,${ }^{13}$ D. Vucinic,${ }^{19}$ R. G. Wagner, ${ }^{1}$ R. L. Wagner, ${ }^{7}$ J. Wahl,${ }^{5}$ N. B. Wallace,${ }^{27}$ A. M. Walsh,${ }^{32}$ C. Wang, ${ }^{6}$ C. H. Wang, ${ }^{33}$ J. Wang, ${ }^{5}$ M. J. Wang, ${ }^{33}$ Q. F. Wang, ${ }^{31}$ A. Warburton, ${ }^{14}$ T. Watts,,${ }^{32}$ R. Webb,${ }^{34}$ C. Wei, ${ }^{6}$ H. Wenzel, ${ }^{16}$ W. C. Wester, III, ${ }^{7}$ A. B. Wicklund, ${ }^{1}$ E. Wicklund,${ }^{7}$ R. Wilkinson, ${ }^{26}$ H. H. Williams, ${ }^{26}$ P. Wilson, ${ }^{5}$ B. L. Winer, ${ }^{23}$ D. Winn,${ }^{20}$ D. Wolinski, ${ }^{20}$ J. Wolinski, ${ }^{21}$ S. Worm,${ }^{22} \mathrm{X}$. Wu ${ }^{10} \mathrm{~J}$. Wyss,${ }^{25}$ A. Yagil,${ }^{7} \mathrm{~W}$. Yao, ${ }^{18} \mathrm{~K}$. Yasuoka, ${ }^{36} \mathrm{Y}$. Ye, ${ }^{14}$ G. P. Yeh,${ }^{7}$ P. Yeh, ${ }^{33}$ M. Yin,${ }^{6}$ J. Yoh,${ }^{7}$ C. Yosef,,${ }^{21}$ T. Yoshida,${ }^{24}$ D. Yovanovitch, ${ }^{7}$ I. Yu, ${ }^{7}$ L. Yu ${ }^{22}$ J. C. Yun, ${ }^{7}$ A. Zanetti, ${ }^{27}$ F. Zetti, ${ }^{27}$ L. Zhang, ${ }^{38}$ W. Zhang, ${ }^{26}$ and S. Zucchelli ${ }^{2}$

\section{(CDF Collaboration)}


1 Argonne National Laboratory, Argonne, Illinois 60439

2 Istituto Nazionale di Fisica Nucleare, University of Bologna, I-40127 Bologna, Italy

3 Brandeis University, Waltham, Massachusetts 02264

4 University of California at Los Angeles, Los Angeles, California 90024

5 University of Chicago, Chicago, Illinois 60638

6 Duke University, Durham, North Carolina 28708

7 Fermi National Accelerator Laboratory, Batavia, Illinois 60510

8 University of Florida, Gainesville, FL 33611

9 Laboratori Nazionali di Frascati, Istituto Nazionale di Fisica Nucleare, I-00044 Frascati, Italy

10 University of Geneva, CH-1211 Geneva 4, Switzerland

11 Harvard University, Cambridge, Massachusetts 02138

12

Hiroshima University, Higashi-Hiroshima 724, Japan

13 University of Illinois, Urbana, Illinois 61801

14

Institute of Particle Physics, McGill University, Montreal H3A 2T8, and University of Toronto,

Toronto M5S 1 A7, Canada

15 The Johns Hopkins University, Baltimore, Maryland 21218

16 Institut für Experimetelle Kernphysik, Universität Karlsruhe, 76128 Karlsruhe, Germany

17 National Laboratory for High Energy Physics (KEK), Tsukuba, Ibaraki 315, Japan

18 Ernest Orlando Lawrence Berkeley National Laboratory, Berkeley, California 94720

19

Massachusetts Institute of Technology, Cambridge, Massachusetts 02139

20 University of Michigan, Ann Arbor, Michigan 48109

21 Michigan State University, East Lansing, Michigan 48824

22 University of New Mexico, Albuquerque, New Mexico 87132

23 The Ohio State University, Columbus, OH 43320

24 Osaka City University, Osaka 588, Japan

25 Universita di Padova, Istituto Nazionale di Fisica Nucleare, Sezione di Padova, I-36132 Padova, Italy

26 University of Pennsylvania, Philadelphia, Pennsylvania 19104

Istituto Nazionale di Fisica Nucleare, University and Scuola Normale Superiore of Pisa, I-56100 Pisa, Italy

28 University of Pittsburgh, Pittsburgh, Pennsylvania 15270

29 Purdue University, West Lafayette, Indiana 47907

30 University of Rochester, Rochester, New York 14628

31 Rockefeller University, New York, New York 10021

32 Rutgers University, Piscataway, New Jersey 08854

33

Academia Sinica, Taipei, Taiwan 11530, Republic of China

34 Texas A\&M University, College Station, Texas 77843

35 Texas Tech University, Lubbock, Texas 79409

36

University of Tsukuba, Tsukuba, Ibaraki 315, Japan

37 Tufts University, Medford, Massachusetts 02155

38 University of Wisconsin, Madison, Wisconsin 53806

39 Yale University, New Haven, Connecticut 06511 


\section{Contents}

Acknowledgements $\quad$ iv

1 Introduction and Theoretical Overview 1

1.1 The Standard Model . . . . . . . . . . . . . . . 4

1.1.1 The CKM Matrix ............... 6

1.2 Bottom — The Fifth Quark . . . . . . . . . . . . 8

1.2.1 $b$ Quark Production .................. 10

1.2.2 b Quark Fragmentation ............... 10

$1.2 .3 \quad$ b Hadron Decay . . . . . . . . . . . . . . . 13

1.3 Particle-Antiparticle Mixing . . . . . . . . . . . . . 14

1.3.1 Neutral $B \bar{B}$ Mixing ................ 17

1.3.2 Mixing and $C P$-Violation . . . . . . . . . 20

1.3.3 Current $B_{d}^{0}$ Mixing Measurements ............ 22

1.4 Overview of the Analysis . . . . . . . . . . . . 23

2 The Tevatron and the CDF Detector 26

2.1 Particle Accelerators at FNAL . . . . . . . . . . . 28

2.1.1 The B0 Interaction Region . . . . . . . . . . . . . 29

2.2 The CDF Detector . . . . . . . . . . . . . . 31

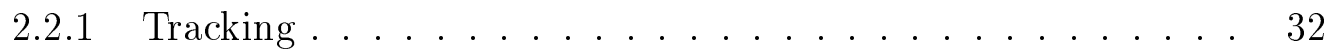


2.2 .2 Calorimetry . . . . . . . . . . . . . . . . . . 40

2.2 .3 Muon Detectors . . . . . . . . . . . . . . . 48

2.2 .4 Trigger System . . . . . . . . . . . . . . . . . 52

3 Primary Datasets $\quad 59$

3.1 The $1994-1995$ Run . . . . . . . . . . . . . . . . . . . . 59

3.2 Event Production . . . . . . . . . . . . . . 60

3.3 BPAD processing $\ldots \ldots \ldots \ldots \ldots \ldots \ldots \ldots \ldots \ldots$

3.3.1 Inclusive electrons (ECLB) $\ldots \ldots \ldots \ldots \ldots$

3.3 .2 Inclusive muons (MULB) $\ldots \ldots \ldots \ldots \ldots$

3.4 Trigger Lepton Selection . . . . . . . . . . . . . . . . 64

3.4.1 Trigger Electron Selection . . . . . . . . . . . . . . . 64

3.4.2 Trigger Muon Selection $\ldots \ldots \ldots \ldots$. . . . . . 67

3.5 Primary Dataset Summary $\ldots \ldots \ldots \ldots$. . . . . . 68

4 Final Data Samples $\quad 69$

$4.1 \quad$ SVX Reprocessing . . . . . . . . . . . . . . . . . . 69

4.2 Primary Vertex Finding $\ldots \ldots \ldots \ldots \ldots \ldots$

4.2 .1 The Seed Vertex . . . . . . . . . . . . . . . 70

4.2 .2 Primary Vertex Fit . . . . . . . . . . . . . . 73

4.3 SECVTX $b$-tagging $\ldots \ldots \ldots \ldots \ldots \ldots \ldots \ldots$

4.3.1 General Algorithm Overview . . . . . . . . 76

4.3 .2 Jet Selection . . . . . . . . . . . . . . . . . 79

4.3 .3 Track Preselection . . . . . . . . . . . . . . . . 79

4.3 .4 PASS 1 Tagging $\ldots \ldots \ldots \ldots \ldots \ldots \ldots$

4.3 .5 PASS 2 Tagging $\ldots \ldots \ldots \ldots \ldots \ldots$

4.3 .6 Candidate Vertexing . . . . . . . . . . . . . 85 
4.4 Event Selection Using SECVTX . . . . . . . . . . . . . 87

4.5 Track-Clustered Jet Definition (TRKSJT) $\ldots \ldots \ldots \ldots$

4.6 Additional Sample Requirements . . . . . . . . . . . . . . . . 89

5 Monte Carlo Samples and Studies $\quad 90$

5.1 Monte Carlo Sample Generation . . . . . . . . . . . . . . 91

5.2 Proper Decay Time $(\mathrm{c} \tau)$ Determination $\ldots \ldots \ldots \ldots$

5.3 Reconstructed $\mathrm{c} \tau$ Efficiency . . . . . . . . . . . . . 96

5.4 Sequential Lepton Fraction $\ldots \ldots \ldots \ldots \ldots$

6 Sample Composition $\quad 103$

6.1 Fake Lepton Samples . . . . . . . . . . . . . . . . . . . . 104

$6.2 p_{\mathrm{t}}^{\mathrm{rel}}$ Method . . . . . . . . . . . . . . . . . . . 109

6.2 .1 Distributions and Fits . . . . . . . . . . . . 109

6.3 Invariant Mass Method . . . . . . . . . . . . . 118

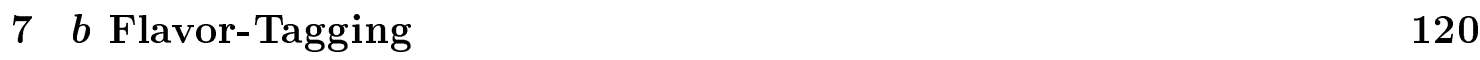

7.1 Flavor-tagging Dilution and Effective Tagging Efficiency $\left(\epsilon D^{2}\right) \ldots 121$

7.2 Soft Lepton Flavor-Tagging . . . . . . . . . . . . . . . . 123

7.2.1 Soft Electron Identification Algorithm . . . . . . . . . 123

7.2.2 Soft Muon Identification Algorithm . . . . . . . . . . . 127

7.2 .3 Soft Lepton Classification . . . . . . . . . . . . . 128

7.2.4 Tag Leptons and Raw Dilution Parametrization . . . . . . . 135

7.2 .5 SLT Systematic Checks _ . . . . . . . . . . . . 151

7.3 Jet Charge Flavor-Tagging . . . . . . . . . . . . . . . . . . 161

7.3.1 Jet Selection and Definition of $Q_{j e t} \ldots \ldots \ldots \ldots$

7.3 .2 Jet Charge Dilutions . . . . . . . . . . . . . . 162

7.3.3 Jet Charge Corrections . . . . . . . . . . . 163 
7.4 Flavor-tagged Subsample Compositions $\ldots \ldots \ldots \ldots \ldots$

7.5 Dilution in $c \bar{c}$ and Fake Events $\ldots \ldots \ldots \ldots \ldots \ldots$

$7.5 .1 \quad c \bar{c}$ Dilution . . . . . . . . . . . . . . . 173

$7.5 .2 \quad$ Fake Dilution . . . . . . . . . . . . . 176

8 Measuring $\Delta m_{d} \quad 179$

8.1 The Maximum-Likelihood Fit . . . . . . . . . . . . . . . 179

8.1.1 The Probability Density . . . . . . . . . . . . . . 181

8.1.2 Convolution with the $\mathrm{c} \tau$ Resolution . . . . . . . 186

8.1 .3 The Total Likelihood . . . . . . . . . . . . . . 188

8.1.4 Fit Parameters . . . . . . . . . . . . . . . . . . . . 189

$8.1 .5 \quad$ Fit Results . . . . . . . . . . . . . . . . . 192

8.1 .6 Fit Algorithm Check . . . . . . . . . . . . . . . . . 194

8.2 Systematic Uncertainties . . . . . . . . . . . . . . . . . . . 199

9 Conclusions 206

$\begin{array}{ll}\text { Bibliography } & 208\end{array}$

A Dilution and "Effective Tagging Efficiency" 213

$\begin{array}{ll}\text { B Determination of } f_{S S}(b \bar{b}) & 220\end{array}$

C $d E / d x$ Corrections $\quad 222$

C.1 A CDF $d E / d x$ Primer . . . . . . . . . . . . . . . . 223

C.2 First Correction Studies With Conversions . . . . . . . . . . . 224

C.2.1 Path-Length Dependence . . . . . . . . . . . 226

C.2.2 Luminosity Dependence . . . . . . . . . . . . . . . 227

C.2.3 NCTC Dependence . . . . . . . . . . . . . . . 228 
C.2.4 First Resolution Estimates . . . . . . . . . . 229

C.3 Aspect Angle Corrections . . . . . . . . . . . . . . . 230

C.3.1 Final Resolution Determination . . . . . . . . . . 231

C.4 The Universal Curve . . . . . . . . . . . . . . . . . 231

C.4.1 Fitting the Curve . . . . . . . . . . . . . . 232

C.5 Polynomial Expansions from the Final Fit . . . . . . . . . . . . 234 


\section{List of Tables}

1.1 Fundamental particles of the Standard Model . . . . . . . . . . . 4

2.1 CTC mechanical and operational parameters . . . . . . . . . 37

$2.2 \mathrm{SVX} / \mathrm{SVX}^{\prime}$ mechanical and operational parameters . . . . . . . . 41

3.1 Trigger electron quality requirements ............. 65

3.2 Conversion partner selection requirements ........... 66

3.3 Trigger muon quality requirements .............. 68

4.1 VXPRIM SVX track requirements . . . . . . . . . . . 74

4.2 SECVTX SVX track preselection requirements . . . . . . . 80

4.3 SECVTX PASS 1 SVX track requirements . . . . . . . . . 82

4.4 SECVTX PASS 2 SVX track requirements . . . . . . . . . . 84

4.5 SECVTX secondary vertex requirements .......... 86

5.1 Sources of trigger leptons in $b \bar{b}$ Monte Carlo . . . . . . . . . . . . . 101

$6.1 f_{b \bar{b}}, f_{c \bar{c}}$, and $f_{f a k e}$ from the $p_{\mathrm{t}}^{\mathrm{rel}}$ fits . . . . . . . . . 114

$6.2 p_{\mathrm{t}}^{\mathrm{rel}}$ fits for various minimum $N_{\mathrm{trk}}^{\mathrm{jet}} \ldots \ldots \ldots \ldots \ldots$

$6.3 f_{b \bar{b}}, f_{c \bar{c}}$, and $f_{f a k e}$ from the $M_{c l}$ fits $\ldots \ldots \ldots 118$

7.1 Soft electron identification criteria . . . . . . . . . 126

7.2 Soft lepton classification . . . . . . . . . . . . . 130 
7.3 Soft electrons in trigger lepton jets . . . . . . . . . . . . . 131

7.4 Projective muons in trigger electron jets . . . . . . . . . . 131

7.5 Projective muons in trigger muon jets . . . . . . . . . . . 132

7.6 Tag electrons in trigger electron data . . . . . . . . . 137

7.7 Tag electrons in trigger muon data $\ldots \ldots \ldots \ldots \ldots$

7.8 Tag muons in trigger electron data (vs. $p_{\mathrm{t}}$ ) $\ldots \ldots \ldots \ldots \ldots$

7.9 Tag muons in trigger muon data $\left(\right.$ vs. $\left.p_{\mathrm{t}}\right) \ldots \ldots \ldots \ldots \ldots$

7.10 Tag muons in trigger electron data (vs. $p_{\mathrm{t}}^{\text {rel }} \& p_{\mathrm{t}}^{\text {comb }}$ ) . . . . . 141

7.11 Tag muons in trigger muon data (vs. $\left.p_{\mathrm{t}}^{\text {rel }} \& p_{\mathrm{t}}^{\text {comb }}\right) \ldots \ldots \ldots 142$

7.12 Tag electron charge asymmetries $\ldots \ldots \ldots \ldots \ldots$

7.13 Tag muon charge asymmetries . . . . . . . . . . . . . . 153

7.14 Tag electrons in Level 2-triggered electron data . . . . . . . . 157

7.15 Tag electrons in Level 2-triggered muon data . . . . . . . . 158

7.16 Tag muons in Level 2-triggered electron data . . . . . . . . . 159

7.17 Tag muons in Level 2-triggered muon data . . . . . . . . . 160

7.18 Sample composition of SLT flavor-tagged events . . . . . . . . . 171

7.19 Sample composition of jet charge flavor-tagged events $\ldots \ldots$. . . 172

8.1 Maximum-likelihood fit parameters . . . . . . . . . . . . . 190

$8.2 \Delta m_{d}$ and $N_{D}$ fit results $\ldots \ldots \ldots \ldots \ldots \ldots \ldots \ldots$

8.3 Systematic uncertainties: SLT flavor-tag (trigger $e$ data) . . . . . 201

8.4 Systematic uncertainties: SLT flavor-tag (trigger $\mu$ data) . . . . . 202

8.5 Systematic uncertainties: jet charge flavor-tag (trigger $e$ data) . . . 203

8.6 Systematic uncertainties: jet charge flavor-tag (trigger $\mu$ data) $\ldots 204$

8.7 Systematic uncertainties for the combined fit $\ldots \ldots \ldots \ldots$ 


\section{List of Figures}

1.1 Feynman diagrams of $b \bar{b}$ production $\ldots \ldots \ldots \ldots$

$1.2 b \bar{b}$ production cross-section . . . . . . . . . . . . . . . 12

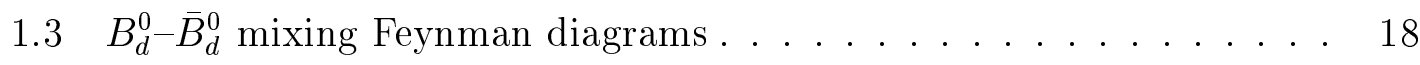

1.4 The Unitarity Triangle $\ldots \ldots \ldots \ldots . \ldots \ldots$

2.1 The FNAL collider complex $\ldots \ldots \ldots \ldots \ldots \ldots$

2.2 Side view of the CDF detector $\ldots \ldots \ldots \ldots \ldots \ldots$

2.3 Perspective view of the CDF detector $\ldots \ldots \ldots \ldots \ldots$

2.4 CTC endplate . . . . . . . . . . . . . . . 37

2.5 Schematic representation of an SVX' barrel . . . . . . . . . 42

2.6 Schematic representation of an SVX' ladder . . . . . . . . . 42

2.7 A central calorimeter wedge $\ldots \ldots \ldots \ldots \ldots \ldots$

2.8 Schematic representation of the CES $\ldots \ldots \ldots \ldots \ldots$

2.9 Muon detector $\eta-\phi$ coverage $\ldots \ldots \ldots \ldots \ldots \ldots$

2.10 Central muon chamber tower $\ldots \ldots \ldots \ldots \ldots \ldots$

$4.1 \mathrm{SVX}^{\prime}$ internal alignment improvement $\ldots \ldots \ldots \ldots \ldots . \ldots 71$

4.2 Illustration of the beampsot size and shape $\ldots \ldots \ldots \ldots$

4.3 SECVTX algorithm flowchart $\ldots \ldots \ldots \ldots \ldots \ldots$

4.4 SECVTX SVX track ordering flowchart . . . . . . . . . 83 
5.1 The $\operatorname{SECVTX} L_{x y}$ bias $\ldots \ldots \ldots \ldots \ldots \ldots \ldots$

$5.2 p_{\mathrm{t}}^{c l} / p_{\mathrm{t}}^{B}$ distributions $\ldots \ldots \ldots \ldots \ldots \ldots \ldots \ldots \ldots \ldots$

$5.3 p_{\mathrm{t}}^{c l}$, true $p_{\mathrm{t}}^{B}$, and corrected $p_{\mathrm{t}}^{\prime}$ distributions $\ldots \ldots \ldots \ldots$

$5.4 \quad$ SECVTX $\mathrm{c} \tau$ efficiency (trigger $e$ data) $\ldots \ldots \ldots \ldots . \ldots 99$

$5.5 \quad$ SECVTX $\mathrm{c} \tau$ efficiency (trigger $\mu$ data) $\ldots \ldots \ldots \ldots$

5.6 Sequential fractions vs. $c \tau \ldots \ldots \ldots \ldots$. . . . . . . . 102

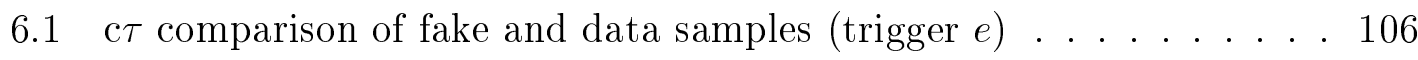

$6.2 \mathrm{c} \tau$ comparison of fake and data samples $($ trigger $\mu$ ) . . . . 107

$6.3 L_{x y}$ comparison of fake and data samples . . . . . . . . 108

6.4 Comparative $N_{\mathrm{trk}}^{\mathrm{jet}}$ distributions $\ldots \ldots \ldots \ldots \ldots \ldots$

6.5 Trigger electron $p_{\mathrm{t}}^{\mathrm{rel}}$ distributions . . . . . . . . . . . . 112

6.6 Trigger muon $p_{\mathrm{t}}^{\text {rel }}$ distributions $\ldots \ldots \ldots \ldots \ldots \ldots \ldots$

$6.7 f_{b \bar{b}}, f_{c \bar{c}}$, and $f_{\text {fake }}$ from the $p_{\mathrm{t}}^{\mathrm{rel}}$ fits $\ldots \ldots \ldots \ldots \ldots \ldots \ldots$

$6.8 p_{\mathrm{t}}^{\mathrm{rel}}$ fits for $N_{\mathrm{trk}}^{\mathrm{jet}} \geq 2$ and $\Delta R=0.8 \ldots \ldots \ldots \ldots \ldots \ldots \ldots \ldots$

6.9 Fake lepton $p_{\mathrm{t}}^{\text {rel }}$ for SECVTX-tagged and -untagged samples $\ldots . .117$

$6.10 M_{c l}$ distributions and fit result for the electron samples . . . . . . . 119

$6.11 M_{c l}$ distributions and fit result for the muon samples $\ldots \ldots \ldots . .119$

7.1 Monte Carlo tag lepton $\Delta R$ and invariant mass $\ldots \ldots \ldots \ldots$

7.2 Soft electron invariant mass distribution . . . . . . . . . 133

7.3 Soft muon invariant mass distribution $\ldots \ldots \ldots \ldots \ldots . \ldots . \ldots 134$

$7.4 \epsilon, D, \epsilon D^{2}$ vs. $p_{\mathrm{t}}$ for soft $e$ and CMU tags (trigger $e$ data) $\ldots \ldots 137$

$7.5 \epsilon, D, \epsilon D^{2}$ vs. $p_{\mathrm{t}}$ for CMP+CMUP and CMX tags (trigger $e$ data) . 138

$7.6 \epsilon, D, \epsilon D^{2}$ vs. $p_{\mathrm{t}}$ for soft $e$ and CMU tags (trigger $\mu$ data) $\ldots \ldots 139$

$7.7 \epsilon, D, \epsilon D^{2}$ vs. $p_{\mathrm{t}}$ for CMP+CMUP and CMX tags (trigger $\mu$ data) $\quad 140$

$7.8 \epsilon, D, \epsilon D^{2}$ vs. $p_{\mathrm{t}}^{\text {rel }}$ for soft $e$ and CMU tags (trigger $e$ data) $\ldots \ldots 143$ 
$7.9 \epsilon, D, \epsilon D^{2}$ vs. $p_{\mathrm{t}}^{\text {rel }}$ for CMP+CMUP and CMX tags (trigger $e$ data) . 143

$7.10 \epsilon, D, \epsilon D^{2}$ vs. $p_{\mathrm{t}}^{\text {rel }}$ for soft $e$ and CMU tags (trigger $\mu$ data) . . . . 144

$7.11 \epsilon, D, \epsilon D^{2}$ vs. $p_{\mathrm{t}}^{\text {rel }}$ for CMP+CMUP and CMX tags (trigger $\mu$ data) . 144

$7.12 \epsilon, D, \epsilon D^{2}$ vs. $p_{\mathrm{t}}^{\text {comb }}$ for soft $e$ and CMU tags (trigger $e$ data) . . . 145

$7.13 \epsilon, D, \epsilon D^{2}$ vs. $p_{\mathrm{t}}^{\text {comb }}$ for CMP+CMUP and CMX tags (trigger $e$ data) 145

$7.14 \epsilon, D, \epsilon D^{2}$ vs. $p_{\mathrm{t}}^{\text {comb }}$ for soft $e$ and CMU tags (trigger $\mu$ data) $\ldots 146$

$7.15 \epsilon, D, \epsilon D^{2}$ vs. $p_{\mathrm{t}}^{\text {comb }}$ for CMP+CMUP and CMX tags (trigger $\mu$ data) 146

7.16 Tag electron raw $D$ vs. $p_{\mathrm{t}}^{\mathrm{rel}} \ldots \ldots \ldots \ldots . \ldots . \ldots 148$

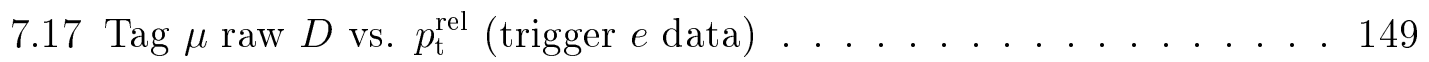

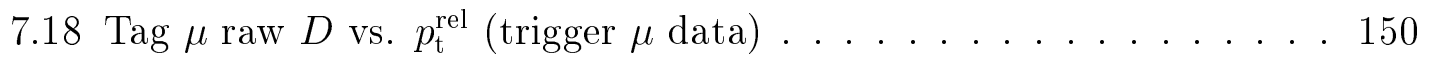

7.19 Tag electron $\epsilon$ and raw $D$ vs. run number . . . . . . . . . 154

7.20 Tag muon $\epsilon$ and raw $D$ vs. run number . . . . . . . . . 154

7.21 Tag electron $\epsilon$ and raw $D$ vs. instantaneous luminosity . . . . . . 155

7.22 Tag muon $\epsilon$ and raw $D$ vs. instantaneous luminosity . . . . . . . 155

$7.23 Q_{\text {jet }}$ distributions for data and $b \bar{b}$ Monte Carlo . . . . . . . . . 164

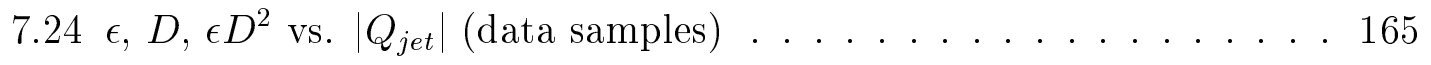

$7.25 \epsilon, D, \epsilon D^{2}$ vs. $\left|Q_{\text {jet }}\right|(b \bar{b}$ Monte Carlo samples $) \ldots \ldots \ldots$

7.26 Charge asymmetry of tracks in opposite side jets . . . . . . . . 168

$7.27 Q_{\text {jet }}$ charge asymmetry correction . . . . . . . . . 169

$7.28 p_{\mathrm{t}}^{\mathrm{rel}}$ fit results for SLT flavor-tagged events . . . . . . . . . . . 171

$7.29 p_{\mathrm{t}}^{\mathrm{rel}}$ fit results for jet charge flavor-tagged events . . . . . . . . . . 172

7.30 Comparative dilutions in $b \bar{b}$ and $c \bar{c}$ Monte Carlo samples . . . . . . . 175

7.31 Comparative dilutions in trigger and fake lepton samples . . . . . . 178

8.1 A generic $p_{\mathrm{t}}^{c l} / p_{\mathrm{t}}^{B}$ distribution $\ldots \ldots \ldots \ldots . \ldots . \ldots . \ldots 187$

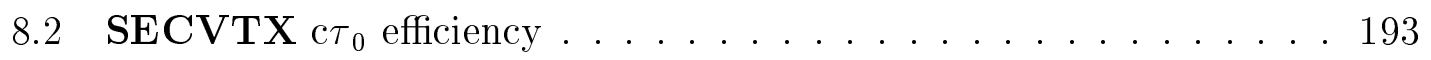

8.3 Individual $\Delta m_{d}$ fit results . . . . . . . . . . . . 195

xviii 
8.4 Combined $\Delta m_{d}$ fit result $\ldots \ldots \ldots \ldots \ldots \ldots \ldots \ldots \ldots$

8.5 Check of $\Delta m_{d}$ and $N_{D}$ fit values and error scales $\ldots \ldots \ldots 197$

8.6 Check of $\log$ likelihood scale . . . . . . . . . . . . . . . 198

C.1 UNQCTC vs. path-length for $p_{\mathrm{t}}>3.0 \mathrm{GeV} / \mathrm{c}$ conversions $\ldots . .235$

C.2 UNQCTC vs. $d \ell d r$, and mean residuals - LO runs . . . . . 235

C.3 Mean residuals vs. instantaneous luminosity . . . . . . . . . 236

C.4 Mean residuals vs. NCTC . . . . . . . . . . . . . . . . 237

C.5 Mean residuals for the full $p_{\mathrm{t}}>3.0 \mathrm{GeV} / \mathrm{c}$ fit $-\mathrm{HI}$ runs $\ldots \ldots 238$

C.6 Fitted width vs. NCTC - MED runs . . . . . . . . . . . . 239

C.7 Full aspc-dependent fit - LO runs . . . . . . . . . . . . 240

C.8 Full aspc-dependent fit - MED runs . . . . . . . . . . . . . 241

C.9 Full aspc-dependent fit - HI runs . . . . . . . . . . . . . 242

C.10 Full aspc-dependent fit $-N_{p h i}-$ LO, MED, and HI runs . . . . 243

C.11 Fitted width vs. NCTC - full fit - LO runs . . . . . . . . . 244

C.12 Fitted width vs. NCTC - full fit - MED runs . . . . . . . . . . 244

C.13 Fitted width vs. NCTC - full fit - HI runs $\ldots \ldots \ldots . . . .244$

C.14 $K_{s}$ candidate mass distribution $\ldots \ldots \ldots \ldots \ldots \ldots$

C.15 $\Lambda^{0}$ candidate mass distribution $\ldots \ldots \ldots \ldots \ldots \ldots$

C.16 $\mathrm{J} / \psi$ candidate mass distribution $\ldots \ldots \ldots \ldots \ldots \ldots$

C.17 QCTC of tracks with $\left|D / \sigma_{D}\right|>100$ - MED runs $\ldots \ldots . . .245$

C.18 Mean corrected QCTC vs. $\beta \gamma-$ LO runs . . . . . . . . 246

C.19 Mean corrected QCTC vs. $\beta \gamma-$ MED runs . . . . . . . . . 246

C.20 Mean corrected QCTC vs. $\beta \gamma-$ HI runs $\ldots \ldots \ldots \ldots . \ldots 246$ 


\section{Chapter 1}

\section{Introduction and Theoretical Overview}

High-energy physics is the study of fundamental particles, and the forces which produce interactions between them. Quite simply, this is effected experimentally by colliding particles together, and observing the results. Particle collisions have a long and rich history; the beginning is usually dated at 1911, by Rutherford's use of $\alpha$-particles from radioactive decay to discover the atomic nucleus [1]. Today, physicists have at their disposal a number of dedicated colliders which accelerate beams of a few types of particles to very high energies, and bring them into collision with static targets or each other. This analysis uses data collected at the Tevatron, a superconducting proton-antiproton collider, and the world's highest-energy particle accelerator.

Theoretical development can be seen as a study of the fundamental symmetries of nature. A system (whether physical or theoretical) has a symmetry if there is a transformation which, when applied to the system, leaves it otherwise identical. Two examples of such symmetries are translations in space and in time. The 
same experiments performed in different locations and on different days produce the same results. In this regard, physical laws are said to be invariant under these translations. Symmetry rules can be very powerful. Einstein's theory of special relativity is based only upon two postulates of invariance - all else follows from them. Gell-Mann and Zweig developed the quark hypothesis to explain near-symmetric patterns in the large number of particles and resonances discovered in the " 50 s and early '60s, bringing theoretical order to experimental chaos.

What can be more exciting than discovering new symmetries is learning that some established symmetries are, in fact, broken. For many years, it was thought that physical laws were invariant under parity $(P)$ transformations - the exchange of left and right, as in a mirror-image. Parity was seen as a fundamental symmetry of space, just as translational and rotational invariance are. In 1956, T.D. Lee and C.N. Yang reviewed the experimental record [2] and concluded that there was no evidence to support parity conservation in weak interactions (those responsible for radioactive decay). C.S. Wu et. al., and others, quickly determined in the following year that the weak force indeed violated parity [3]; Frauenfelder et. al., found [4] that parity was maximally violated. This was confirmed by M. Goldhaber, L. Grodzins, and A.W. Sunyar, who observed [5] that neutrinos produced in beta-decay have only left-handed helicity. Until 1964, it was thought that charge-conjugation $(C$, the exchange of particle and antiparticle) combined with parity $(C P)$ was a good symmetry. In that year, Christenson, Cronin, Fitch, and Turlay observed a rare, $C P$-violating decay of the neutral kaon [6].

The Standard Model was developed to describe these and other discoveries in the context of a relativistic field theory. It is stunningly successful, the capstone of a century of physics. Nevertheless, many parameters of the Standard Model are not very well known, or not known at all. For example, $C P$-violation has not yet 
been studied enough to determine if the Standard Model is a correct account of the symmetry-breaking.

The analysis presented in this dissertation is part of an effort by the CDF collaboration (and many others) to measure the parameters of the Standard Model. The neutral $B$ mesons can transform into their own antiparticles through a secondorder weak interaction. The rate at which the particle and antiparticle states mix depends critically on the weak coupling between quarks. This process is discussed in much more detail in the following sections. Mixing is of great interest, as it is fundamentally related to the mechanics of $C P$-violation, and also serves as a test for physics beyond the Standard Model that cannot be measured directly, owing to technological limitations.

Many of the experimental techniques used by this analysis are also used in $C P$-violation measurements. The ability of CDF to measure $B_{d}^{0}-\bar{B}_{d}^{0}$ oscillations is not only a direct test of the Standard Model, but also a demonstration of CDF's potential to measure $C P$-violation in the future. On this, the 100th anniversary of the discovery of the electron, it is an exciting time to participate in a research program which is (with a touch of immodesty) a small part of one of mankind's greatest intellectual achievements. 


\begin{tabular}{|ccc|ccc|c|}
\hline \multicolumn{9}{|c|}{ Fermions } & Bosons \\
\hline Quarks & $\mathrm{Q}$ & \multicolumn{2}{|c|}{ Leptons } & $\mathrm{Q}$ & $W^{ \pm}, Z^{0}, \gamma$, \\
gluons $(\mathrm{g})$, \\
down strange bottom & $-1 / 3$ & $e$ & $\mu$ & $\tau$ & -1 & Higgs $(\mathrm{H})$ \\
\hline All fermions have antiparticle counterparts \\
Leptons are colorless \\
Quarks come in 3 colors (red, green, blue) \\
Gluons come in 8 color-anticolor combinations \\
Neutrinos, photons, and gluons are massless \\
\hline
\end{tabular}

Table 1.1: Fundamental particles of the Standard Model. The electric charge Q is given in units of the absolute electron charge $(|e|)$.

\subsection{The Standard Model}

The Standard Model describes the fundamental particles and their interactions. Table 1.1 highlights several aspects of the theory ${ }^{1}$. The material world is constructed of fermions, having odd half-integer spin. The fermions can be cataloged by which of the fundamental forces affect them. The bosons, having integer spin, mediate the interactive forces between the fermions, and can bind them together to form composite particles.

There are six leptons, arranged in 3 generations (also called families). Each generation has a charged and a neutral lepton. The lightest charged lepton is the electron. The neutral leptons (neutrinos) can only interact via the weak force, and are therefore only left-handed. Electroweak theory [7] describes the unification of electromagnetism and the weak force through the Higgs mechanism [8]. The electroweak gauge group is $\mathrm{SU}(2)_{\mathrm{L}} \otimes \mathrm{U}(1)_{\mathrm{Y}}$. The electroweak force conserves lepton

\footnotetext{
${ }^{1}$ Unless specifically noted or obvious from context, references to a specific charge state should be understood to also refer to the charge conjugate state.
} 
number $^{2}$ - it cannot transform leptons from one doublet to those of another. Neutral current interactions via the $Z^{0}$ do not change the type of lepton; charged $W$ exchange can only transform leptons within doublets. Thus the decay $\mu^{-} \rightarrow e^{-} \bar{\nu}_{e} \nu_{\mu}$ is allowed, while $\pi^{0} \rightarrow \mu^{ \pm} e^{\mp}$ is not. Lepton number conservation is a consequence of the three neutrinos having the same mass (zero, in the case of the Standard Model). Nonzero neutrino masses, however, have not been ruled out experimentally ${ }^{3}$, so it is possible that lepton number is not conserved. The Higgs boson, which breaks the electroweak symmetry by coupling to the mass of particles, has not been experimentally observed and is much sought-after: it is the only particle predicted by the Standard Model which has not been observed.

There are six quarks, arranged in three generations of doublets like the leptons. They carry color charge and interact predominantly via the strong force. The six types of quarks are commonly referred to as their flavors. The strong interaction, mediated by gluons, is described by the SU(3) group symmetry of Quantum Chromodynamics (QCD) [9]. Each quark doublet is composed of a quark with $+2 / 3$ electrical charge ( $u$-type) and one with $-1 / 3$ electrical charge ( $d$-type). Like the leptons, this grouping reflects how the members of each doublet can be transformed into one another by $W$ exchange. Unlike the leptons, however, the weak force does not completely conserve a quark "family number". In the electroweak basis, there is a slight "rotation" of the quark generations relative to the QCD/flavor basis. The convention is that the rotation occurs among $d$-type quarks, allowing them to couple with any of the $u$-type quarks via $W$ exchange. The rotation is allowed because the $d$-type quarks have different masses. The matrix which describes the basis

\footnotetext{
${ }^{2}$ More correctly, there are three kinds of lepton number (one for each family), each of which is independently conserved.

${ }^{3}$ In fact, measurements [10] of the flux of solar neutrinos suggest that electron neutrinos may have a nonzero, though very small, mass.
} 
transformation is known as the Cabbibo-Kobayashi-Maskawa (CKM) mixing matrix $[11,12]$. It is through this flavor rotation that a second-order weak interaction can transform a $B_{d}^{0}$ meson into a $\bar{B}_{d}^{0}$ meson. Consequently, mixing measurements constitute determinations of the CKM matrix parameters.

Quarks, unlike leptons, have not been seen as free particles. They exist only in bound states, with other quarks, called hadrons. QCD's principles of asymptotic freedom and color confinement predict that particles with color charge (like quarks) must combine with others to form "colorless" color-singlet composite particles. There are two main classes of hadrons: mesons are bound states of a quark and an antiquark (color + anticolor $=$ colorless $)$; baryons are bound states of three quarks $($ red + blue + green $=$ colorless $)$.

\subsubsection{The CKM Matrix}

Until the discovery of the charm quark in 1974 [13], the known hadrons fit into the Gell-Mann and Zweig theoretical model which contained only 3 quarks $(u, d$, and $s)$. The weak decays involved a left-handed doublet $\left(u_{L}, d_{L}^{\prime}=d \cos \theta_{C}+s \sin \theta_{C}\right)$ and a singlet $\left(s_{L}^{\prime}=s \cos \theta_{C}-d \sin \theta_{C}\right)$, where $\theta_{C}$ is the Cabibbo angle [11] — the measure of the $d-s$ mixing. In this model, one would expect to observe neutral strangeness-changing decays like $K_{L}^{0} \rightarrow \mu^{+} \mu^{-}$with an amplitude, proportional to $+\cos \theta_{C} \sin \theta_{C}$, which is at least competitive with the $K_{\mu 3}^{0} \operatorname{mode}\left(K_{L}^{0} \rightarrow \pi^{ \pm} \mu^{\mp} \nu\right)$. The decay was not observed, and by 1970 the experimental limit was orders of magnitude smaller than the expected rate $^{4}$.

In that year, Glashow, Iliopoulos, and Maiani proposed an explanation for the nonobservation [14]. The "GIM mechanism" requires the existence of a fourth quark,

\footnotetext{
${ }^{4}$ The branching ratio $\mathrm{BR}\left(K_{L}^{0} \rightarrow \mu^{+} \mu^{-}\right)$is currently measured [15] to be $(7.2 \pm 0.5) \times 10^{-9}$, as compared to $\operatorname{BR}\left(K_{L}^{0} \rightarrow \pi^{ \pm} \mu^{\mp} \nu\right)=0.270 \pm 0.004$.
} 
charm, to form a doublet with the strange quark. This creates another contribution to $K_{L}^{0} \rightarrow \mu^{+} \mu^{-}$, with an amplitude proportional to $-\cos \theta_{C} \sin \theta_{C}$, which almost completely cancels out the contribution above ${ }^{5}$. The Cabbibo angle $\theta_{C}$ can then be seen as the rotation of the $d$ and $s$ quark between the mass eigenstates and the weak eigenstates. The 1974 discovery of the $J / \psi$ provided evidence for the existence of the charm quark, and the discovery of the $D$ mesons in 1976 gave proof [16].

Even before these observations, theorists explored the extension of the number of weak doublets to three [12] or more. In a theory with three generations, the quark mixing is given by the Cabibbo-Kobayashi-Maskawa (CKM) matrix:

$$
\left(\begin{array}{c}
d^{\prime} \\
s^{\prime} \\
b^{\prime}
\end{array}\right)=\left(\begin{array}{ccc}
V_{u d} & V_{u s} & V_{u b} \\
V_{c d} & V_{c s} & V_{c b} \\
V_{t d} & V_{t s} & V_{t b}
\end{array}\right)\left(\begin{array}{c}
d \\
s \\
b
\end{array}\right)
$$

The elements $V_{i j}$ of the matrix represent the coupling between a $u$-type quark and a $d$-type quark. This generalized matrix must be unitary. The elements $V_{u d}$ and $V_{u s}$ represent $\cos \theta_{C}$ and $\sin \theta_{C}$, respectively. A convenient parametrization [17] of the CKM matrix, due to Wolfenstein, is:

$$
V \sim\left(\begin{array}{ccc}
1-\lambda^{2} / 2 & \lambda & A \lambda^{3}(\rho-i \eta) \\
-\lambda & 1-\lambda^{2} / 2 & A \lambda^{2} \\
A \lambda^{3}(1-\rho-i \eta) & -A \lambda^{2} & 1
\end{array}\right)
$$

where the parameters are $\lambda \approx 0.22, A \approx 0.90 \pm 0.12$, and $\sqrt{\rho^{2}+\eta^{2}} \approx 0.39 \pm 0.07[17$, 18]. The experimentally allowed values [19] for the matrix elements, allowing for

\footnotetext{
${ }^{5}$ The cancellation would be perfect if the $u$ and $c$ quarks had the same mass.
} 
the possibility of more than three generations, are

$$
\left(\begin{array}{cccc}
0.9720-0.9752 & 0.217-0.223 & 0.002-0.005 & \ldots \\
0.199-0.234 & 0.818-0.975 & 0.036-0.046 & \ldots \\
0-0.11 & 0-0.52 & 0-0.9993 & \ldots \\
\vdots & \vdots & \vdots & \ddots
\end{array}\right)
$$

The most interesting feature of the CKM matrix is that it contains a complex phase $\eta$. The matrix entries in general are complex, but unitarity places bounds on the number of real angles and imaginary phases. For $n$ generations, there are $n(n-1) / 2$ angles and $(n-1)(n-2) / 2$ phases. The three-generation CKM matrix therefore has 3 angles and one phase, as shown in Equation 1.2. A non-zero value for the phase allows the electroweak force to break $C P$-invariance.

\subsection{Bottom - The Fifth Quark}

In a theory with only two generations, the CKM matrix elements would consist of one real angle, the Cabbibo angle, and $C P$ would be an unbroken symmetry. The development of the three-generation CKM matrix was well-received, as $C P$ was known to be broken by $K^{0}$ decays. The first direct experimental evidence for the third generation was in 1975, with the discovery of the tau lepton [20], an achievement which led to a Nobel Prize.

With a third lepton must come a third pair of quarks, bottom and top ${ }^{6}$. As the strange quark is less massive than the charm quark, it was expected that bottom, with $-1 / 3$ charge, would be found first. Evidence of bottom production was first obtained in 1977 , by the discovery [21] of a $\sim 9.5 \mathrm{GeV} / \mathrm{c}^{2}$ resonance in $\mu^{+} \mu^{-}$pairs

\footnotetext{
${ }^{6}$ The alternate names "beauty" and "truth" can be found in the literature, for the more poetically inclined.
} 
produced by $400 \mathrm{GeV}$ protons incident on a fixed target. Shortly thereafter, a more detailed analysis of the data found that the "resonance" was actually two narrow resonances, lying close together at $9.44 \mathrm{GeV} / \mathrm{c}^{2}$ and $10.17 \mathrm{GeV} / \mathrm{c}^{2}$. These resonances, which are named the $\Upsilon$ and $\Upsilon^{\prime}$, became understood to be bound states of bottom quark-antiquark pairs, much as the $J / \psi$ and $\psi^{\prime}$ are the bound states of charm quark-antiquark pairs. The measurement of the partial width for $\Upsilon \rightarrow$ $e^{+} e^{-}$, coupled with a potential model derived from the $J / \psi$, strongly supported the hypothesis that the new quark had a charge of $-1 / 3$ [22].

In 1980, experiments at the Cornell Electron Storage Ring (CESR) observed the $\Upsilon$ and $\Upsilon^{\prime}$, and two new resonances, the $\Upsilon^{\prime \prime}$ at $10.36 \mathrm{GeV} / \mathrm{c}^{2}$ and the $\Upsilon^{\prime \prime \prime}$ at $10.58 \mathrm{GeV} / \mathrm{c}^{2}$ [23]. The $\Upsilon^{\prime \prime \prime}$, also known as the $\Upsilon(4 \mathrm{~S})$, is unlike the three lighter resonances, which are narrow. The $\Upsilon(4 \mathrm{~S})$ is fairly broad. Following experience with the $\psi^{\prime \prime}$, the obvious interpretation was that the mass of the $\Upsilon(4 \mathrm{~S})$ is above threshold for $B \bar{B}$ production, where the $B$ is a meson containing a $b$ antiquark and a lighter quark. Since the discovery of the $\Upsilon(4 \mathrm{~S}), B$ mesons have been studied at dedicated $e^{+} e^{-}$colliders with center-of-mass energies tuned to the peak of the $\Upsilon(4 \mathrm{~S})$ resonance.

$b$ hadrons can also be studied in other environments. $e^{+} e^{-}$colliders tuned to the $Z^{0}$ mass produce $b \bar{b}$ pairs in large quantites (the $Z^{0}$ decays into bottom quarks approximately $15 \%$ of the time [19]). So do $p \bar{p}$ colliders and proton fixed-target experiments, which produce them through strong interactions between the quark and gluon constituents of the nucleons. Unlike the $e^{+} e^{-}$colliders tuned to the $\Upsilon(4 \mathrm{~S})$, however, the $b$ hadrons produced by these experiments can be any species, including the $B_{s}^{0}$ meson and $b$ baryons $\left(\Lambda_{b}\right)$, as the the center-of-mass energies are typically much larger than the $b$ mass. The higher energy also assists in measuring the $b$ hadron lifetimes, which are approximately 1.5 ps. $B$ mesons from $\Upsilon(4 \mathrm{~S}) \rightarrow B \bar{B}$ are 
produced nearly at rest. They do not travel far in the laboratory before they decay, making a measurement of their lifetime difficult. The more energetic $b$ hadrons produced by $Z^{0}$ decay or in hadronic collisions travel a measurable distance before decaying. This fact can also be used to preferentially select collisions that contain $b$ hadrons, providing the necessary background rejection for a host of different measurements of $b$ hadron production and decay.

\subsection{1 $b$ Quark Production}

In $p \bar{p}$ collisions, the constituent $u$ and $d$ quarks and gluons from the proton and antiproton interact, producing $b \bar{b}$ quark-antiquark pairs. In Figure 1.1, several Feynman diagrams contributing to this process are shown. A next-to-leading order QCD calculation of $b \bar{b}$ production has been performed [24]. The theoretical prediction for the $b \bar{b}$ production cross-section in $p \bar{p}$ collisions, as a function of the quark $p_{\mathrm{t}}$, can be derived from this calculation and a set of parton distribution functions [25] describing the kinematics of the quark and gluon constituents of protons. Figure 1.2 shows the predicted cross-section along with a number of measurements from the CDF data.

\subsection{2 $b$ Quark Fragmentation}

Quarks have not been detected as free particles. QCD's principle of color confinement predicts that quarks must bind together with other quarks and gluons to form color-singlet hadrons. As the $b$ and $\bar{b}$ quarks separate, they radiate gluons, producing quark-antiquark pairs that bind to the $b$ quarks, and each other, to yield a jet of hadrons. This process is called fragmentation. As it is a low momentumtransfer process, it is not well-decribed by perturbative QCD. An empirical model of fragmentation, based on kinematic arguments, has been developed by Peterson 
Figure 1.1: Feynman diagrams contributing to $b \bar{b}$ production in a $p \bar{p}$ collider environment. 


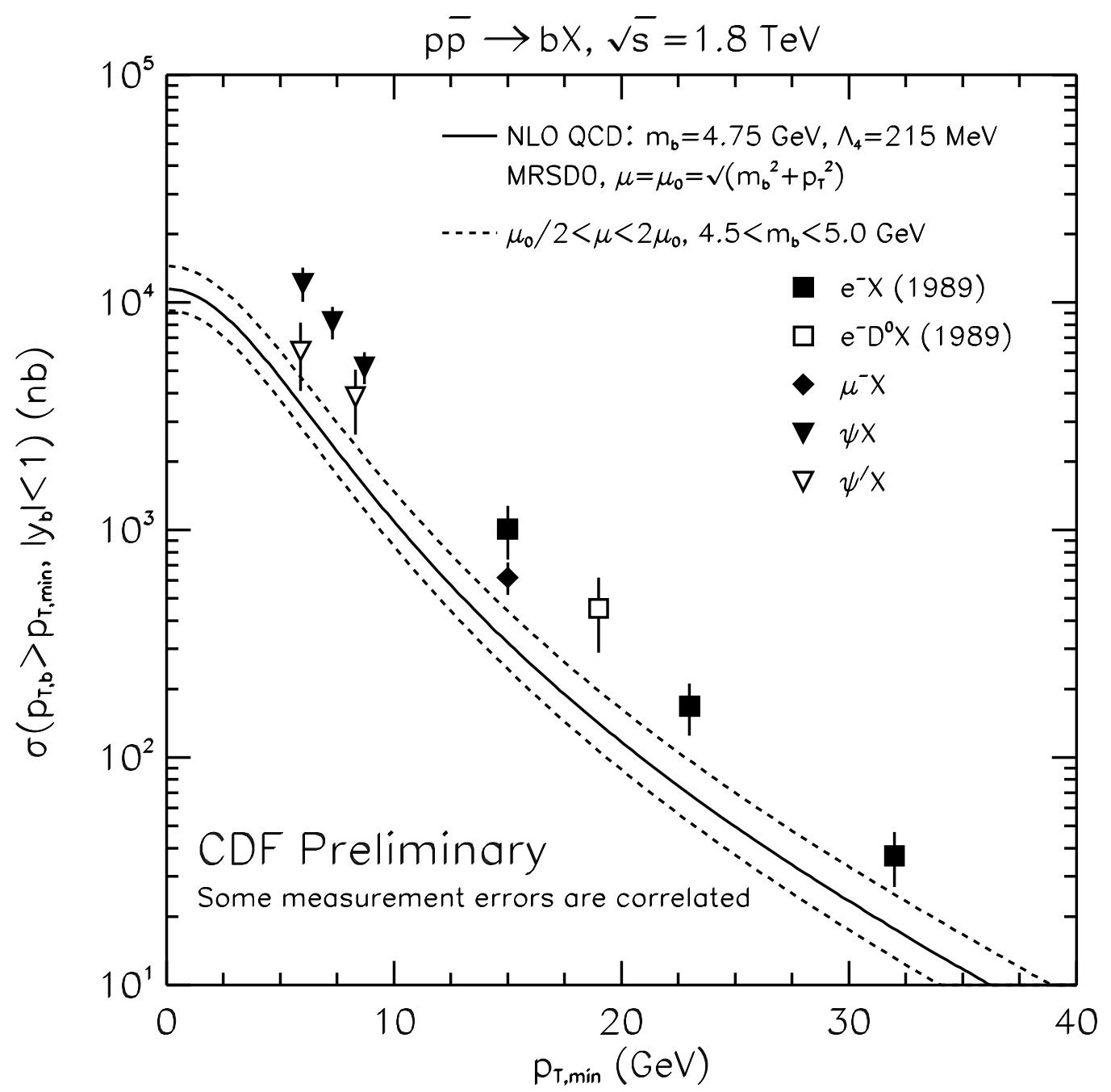

Figure 1.2: The $b \bar{b}$ production cross-section, comparing CDF experimental results to a range of theoretical predictions. 
and others [26]. The model parametrizes the fraction of the $b$ quark momentum carried by the hadrons in the jet to the form

$$
D_{q}^{H}(z)=K \frac{1}{z}\left(1-\frac{1}{z}-\frac{\epsilon_{q}}{1-z}\right)^{-2}
$$

where $D_{q}^{H}$ is the fragmentation function (the probability for a quark of type $q$ to produce a hadron of type $H$ ), $z$ is the fraction of the $b$ quark momentum carried by the hadron, $K$ is a constant, and $\epsilon_{q}$ is the Peterson parameter. $\epsilon_{b}$ has been determined to be $0.006 \pm 0.002$, and $\epsilon_{c}$ to be $0.06_{-0.02}^{+0.03}$ [19]. On average, a $b$ hadron carries approximatexly $70 \%$ of the momentum of its parent $b$ quark. Charmed hadrons carry a lower fraction of their parent's momentum, approximately 50\%, suggesting that the number of fragmentation particles in a typical jet will be lower for $b$ jets, as less energy is available to create them.

The fragmentation process also determines the particular species of the hadrons. As $u$ and $d$ are the lightest quarks, the $B_{u}^{-}$and $B_{d}^{0}$ are produced in the largest numbers. $B_{s}^{0}$ production is suppressed owing to the larger mass of the $s$ quark; $b$ baryon production $\left(\Lambda_{b}\right)$, which requires two quarks to be added to the $b$, is also suppressed. $B_{c}^{-}$production is negligible compared to all these. A measurement of the species production ratios can be made from studies of the relative abundances of pions, kaons, and protons in $b$ jets. The current values [19] are $B_{u}^{-}: B_{d}^{0}: B_{s}^{0}: \Lambda_{b}=37.8 \%: 37.8 \%: 11.2 \%: 13.2 \%$.

\subsection{3 $b$ Hadron Decay}

Unlike protons, $b$ hadrons are not stable particles. They quickly decay into other particles. $b$ hadron decay is well-described by the spectator model, in which the primary decay process is the emission of a virtual $W$ from the $b$ quark, transforming 
it into either a $c$ or a $u$ quark. The lighter quark(s) in the $b$ hadron apppear in both initial and final states, and play little role in the decay except for some moderation via soft gluon exchange. Decay of charged $B$ mesons by annihilation to a virtual $W$ has not been observed, and is only considered competitive with spectator model decay modes for $B_{c}^{-}$decays. The decay width for $b \rightarrow W q$ is given by

$$
\Gamma_{b \rightarrow W q}=\frac{G_{F}^{2} m_{b}^{5}}{192 \pi^{3}} \cdot\left|V_{q b}\right|^{2} \cdot F\left(\frac{m_{q}}{m_{b}}\right)
$$

where $G_{F}=1.17 \times 10^{-5} \mathrm{GeV}^{-2}$ is the weak Fermi constant, $V_{q b}$ is the CKM matrix element (where $q$ can be $c$ or $u$ ), and $m_{b}, m_{q}$ are the quark masses. $F$ is the product of a phase space correction and a QCD correction [27]; it is smaller for higher $m_{q}$, as less momentum is available for the decay products.

\subsection{Particle-Antiparticle Mixing}

A description of the neutral kaon system will serve as an introduction to particleantiparticle mixing. The $K^{0}$ meson, composed of $d \bar{s}$, is an interesting particle from a physical and historical perspective. It was the first neutral boson thought not to be its own antiparticle, an idea which met with some resistance when first proposed by Gell-Mann in 1953. Subsequent measurements of hadronic production demonstrated that it was true, and a number of similar neutral mesons have been discovered to date.

Most particles have some characteristic which readily distinguishes them from their antiparticles. Electrically-charged particles are obviously distinct. For the neutrals, there can be helicity (for neutrinos) or baryon number (for antineutrons). Some neutral particles are their own antiparticle, leaving no distinction to be made. 
But in the neutral kaon, the world had found something subtle: the $K^{0}$ is distinguished from the $\bar{K}^{0}$ only by its flavor. As kaons only decay by the weak force, which does not conserve flavor, this distinction does not amount to a very large difference.

The $K^{0}$ and $\bar{K}^{0}$ can decay to the same two- and three-pion final states. Thus the two particles can transform into one another via virtual pion intermediate states. This process is called mixing. It is best understood in the context of exact $C P$ invariance.

Because $K$ s are strongly produced, they are created in flavor eigenstates. The flavor symmetry is broken when they decay weakly. The physical states (those with definite masses and lifetimes) must be eigenstates of $C P$. Under $C P$, the neutral kaons are transformed into their antiparticles:

$$
C P\left|K^{0}\right\rangle=-\left|\bar{K}^{0}\right\rangle ; \quad C P\left|\bar{K}^{0}\right\rangle=-\left|K^{0}\right\rangle
$$

where the minus signs establish the (arbitrary) phase convention. The $C P$ eigenstates are clearly not the flavor eigenstates; instead, they are

$$
\begin{aligned}
& \left|K_{1}^{0}\right\rangle=\frac{1}{\sqrt{2}}\left[\left|K^{0}\right\rangle-\left|\bar{K}^{0}\right\rangle\right] \\
& \left|K_{2}^{0}\right\rangle=\frac{1}{\sqrt{2}}\left[\left|K^{0}\right\rangle+\left|\bar{K}^{0}\right\rangle\right]
\end{aligned}
$$

where $K_{1}^{0}$ has eigenvalue +1 and $K_{2}^{0},-1$.

Of the hadronic states to which the neutral kaons decay, $\pi^{+} \pi^{-}$and $\pi^{0} \pi^{0}$ have $C P=+1 ; \pi^{+} \pi^{-} \pi^{0}$ and $\pi^{0} \pi^{0} \pi^{0}$ are dominated by $C P=-1$ (a portion of $C P=+1$ is allowed via highly-supressed states of relative angular momentum between the three pions). Insofar as $C P$ is assumed to be a perfect symmetry, it is impossible for the decay $K_{2}^{0} \rightarrow \pi \pi$ to proceed. Since three pion masses are very close to the 
$K^{0}$ mass, the lifetime of the $K_{2}^{0}$ will be much longer than the $K_{1}^{0}$. For obvious reasons, $K_{1}^{0}$ is called $K^{0}$-short $\left(K_{S}^{0}\right)$ and $K_{2}^{0}$ is called $K^{0}$-long $\left(K_{L}^{0}\right)$. The $K_{L}^{0}$ was first observed in 1956 by Lande et. al. [28].

The time development of a particle which is a $K^{0}$ at time $t=0$ can be described by the plane-wave solutions to the Schrödinger equation:

$$
\begin{aligned}
|K(t)\rangle & =a(t)\left|K^{0}\right\rangle+\bar{a}(t)\left|\bar{K}^{0}\right\rangle \\
a(t) & =\frac{1}{2}\left[e^{-i m_{1} t-\frac{1}{2} \Gamma_{1} t}+e^{-i m_{2} t-\frac{1}{2} \Gamma_{2} t}\right] \\
\bar{a}(t) & =\frac{1}{2}\left[-e^{-i m_{1} t-\frac{1}{2} \Gamma_{1} t}+e^{-i m_{2} t-\frac{1}{2} \Gamma_{2} t}\right]
\end{aligned}
$$

where $m_{1}, \Gamma_{1}$ are the mass and width of the $K_{1}^{0}$, and likewise $m_{2}, \Gamma_{2}$ for the $K_{2}^{0}$. A convenient reformulation is $\Gamma \equiv \frac{1}{2}\left(\Gamma_{1}+\Gamma_{2}\right), \Delta \Gamma \equiv \Gamma_{1}-\Gamma_{2}$, and similarly for the masses. Then the amplitudes of Equation 1.9 can be expressed with the Hamiltonian matrix

$$
i \frac{d}{d t}\left(\begin{array}{c}
a \\
\bar{a}
\end{array}\right)=\left(\begin{array}{cc}
m-\frac{1}{2} i \Gamma & \Delta m-\frac{1}{2} i \Delta \Gamma \\
\Delta m^{*}-\frac{1}{2} i \Delta \Gamma^{*} & m-\frac{1}{2} i \Gamma
\end{array}\right)\left(\begin{array}{c}
a \\
\bar{a}
\end{array}\right)
$$

The equality of the diagonal elements and that the off-diagonal elements are complex conjugates of each other follow from the Hermitian properties of the Hamiltonian and general $C P T$ invariance. The elements of the matrix are best understood in this manner: the mass $m$ is given by the consituent quark masses and the QCD meson binding energy; the width $\Gamma$ is from $K^{0} \rightarrow X, \bar{K}^{0} \rightarrow \bar{X}$ decays where $X \neq \bar{X}$; those decays where $X=\bar{X}$ contribute to $\Delta \Gamma$; and $\Delta m$ is generated by direct quark weak box diagrams of a type that will be discussed in the context of $B \bar{B}$ mixing (though see Figure 1.3). In the case of kaon mixing, $\Delta m$ is much smaller than $\Delta \Gamma$, as the kinematics of the two- and three-pion decay modes create such a large difference between the $K_{S}^{0}$ and $K_{L}^{0}$ lifetimes. 
The mixing oscillation frequency "competes" with the decay time: if the neutral kaons mix faster than they decay, then any $K^{0}$ becomes a 50:50 mixture of $K^{0}: \bar{K}^{0}$; if the $K^{0} \mathrm{~s}$ decay faster than they can mix, then mixing will not measurably contribute to their time-development ${ }^{7}$. This is easily shown via the time-integrated mixing probabilities derived from Equations 1.9 or 1.12 :

$$
\begin{aligned}
P\left(K^{0} \rightarrow K^{0}\right) & \equiv \int|a(t)|^{2} d t=\frac{\frac{1}{2}\left[(\Delta m)^{2}+\frac{1}{4}(\Delta \Gamma)^{2}\right]}{(\Delta m)^{2}+\Gamma^{2}} \\
P\left(K^{0} \rightarrow \bar{K}^{0}\right) & \equiv \int|\bar{a}(t)|^{2} d t=\frac{\frac{1}{2}\left[(\Delta m)^{2}+2 \Gamma-\frac{1}{4}(\Delta \Gamma)^{2}\right]}{(\Delta m)^{2}+\Gamma^{2}}
\end{aligned}
$$

\subsubsection{Neutral $B \bar{B}$ Mixing}

The equations governing $B \bar{B}$ mixing are identical to those for $K \bar{K}$ mixing, but they arise from different processes. Whereas $K^{0}-\bar{K}^{0}$ transitions are mediated by virtual pion decay states that both particle and antiparticle have in common, neutral $B$ mesons decay primarily to charmed mesons, leaving the flavor of $B_{d}^{0}$ and $\bar{B}_{d}^{0}$ decay products distinguishable. The largest contribution to the common decay modes is $B_{d}^{0}, \bar{B}_{d}^{0} \rightarrow J / \psi \pi^{0}$, suppressed by phase space and a factor of $\left|V_{c d}\right|$, with a partial width less than $7 \times 10^{-3}$ of the total decay width [19]. Thus $\Delta \Gamma$ between the $C P$ eigenstates is very small, and can be assumed to be zero for this treatment. The mixing is dominated by $\Delta m$. Recall that $\Delta m$ is created by virtual weak interactions between the quark constituents. The Feynman diagrams for these processes are shown in Figure 1.3.

\footnotetext{
${ }^{7}$ This is the case with the neutral charmed mesons. The $D^{0}$ lifetime is much shorter than the predicted mixing oscillation time. Not surprisingly, $D^{0}-\bar{D}^{0}$ mixing has not been observed.
} 

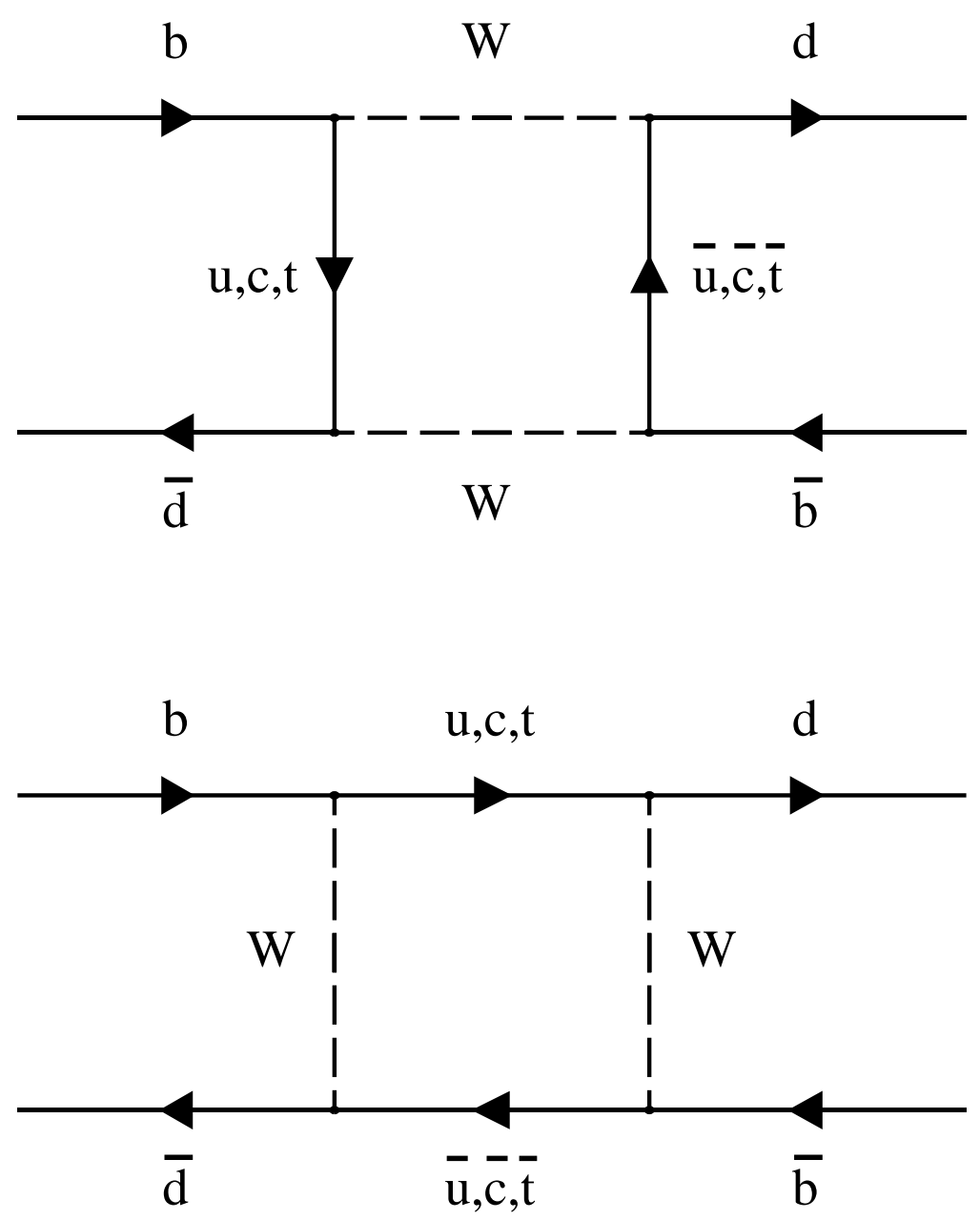

Figure 1.3: Feynman diagrams for $B_{d}^{0}$ mixing. The quark exchanges are dominated by the $t$ quark, owing to its large mass. The same diagrams are applicable to the $B_{s}^{0}$, with $s$ quarks in place of the $d$ quarks, and the $K^{0}$, with $s$ quarks in place of the $b$ quarks. 
As $\Delta \Gamma \approx 0$, the solutions to Equation 1.12 can be written as

$$
\begin{aligned}
|a(t)|^{2} & =\frac{1}{2} e^{-\Gamma t}[1+\cos \Delta m t] \\
|\bar{a}(t)|^{2} & =\frac{1}{2} e^{-\Gamma t}[1-\cos \Delta m t]
\end{aligned}
$$

leading to time-integrated mixing probabilities of

$$
r \equiv \frac{P(B \rightarrow \bar{B})}{P(B \rightarrow B)}=\frac{2(\Delta m)^{2}}{\Gamma^{2}+2(\Delta m)^{2}}
$$

A convenient variable is $x \equiv \frac{\Delta m}{\Gamma}$.

For $B_{d}^{0}$ mixing, $\Delta m_{d}$ can be calculated from the box diagrams of Figure 1.3. In the Standard Model, the dominant diagram is when both virtual quarks are top quarks: the amplitude increases with the mass, and top quarks are heaviest of all. In this way, mixing measurements can probe much higher mass scales for new physics than can be searched directly by accelerators. Should there be an undiscovered, very heavy charged particle which can take the place of the three known $u$-type quarks in Figure 1.3, it can come to dominate the mixing amplitude, if its coupling to $b$ quarks is sufficiently large. A charged Higgs boson, from theories with two Higgs multiplets, could play this role. In the Standard Model, the prediction [29] for $\Delta m_{d}$ is

$$
\Delta m_{d}=\frac{G_{F}^{2} m_{B_{d}^{0}}}{6 \pi^{2}} f_{B_{d}^{0}}^{2} B_{B_{d}^{0}}\left|V_{t d} V_{t b}^{*}\right|^{2}\left[m_{W}^{2} S\left(\frac{m_{t}}{m_{W}}\right)\right] \eta_{Q C D}
$$

$G_{F}$ is the weak Fermi constant and $V_{i j}$ are the CKM matrix elements. $m_{B_{d}^{0}}, m_{t}$, and $m_{W}$ are the measured $B_{d}^{0}$ meson, top quark, and $W$ boson masses, respectively. $f_{B_{d}^{0}} B_{B_{d}^{0}}^{1 / 2}=200 \pm 40 \mathrm{MeV}$ is the product of the leptonic decay constant and the "bag" factor, calculated for the $B_{d}^{0}$ meson using lattice QCD and QCD sum rules [30]. 
The Inami-Lim function [31] $S\left(m_{t} / m_{W}\right) \approx 0.784\left(m_{t} / m_{W}\right)^{1.52} \approx 2.46 \pm 0.13$ is the amplitude of the box integral. The QCD correction factor $\eta_{Q C D} \approx 0.55 \pm 0.01$ is estimated by [32]

$$
\eta_{Q C D}=X^{-\frac{6}{23}} \cdot\left[\frac{3}{2} Y^{-\frac{4}{7}}-Y^{-\frac{2}{7}}+\frac{1}{2} Y^{\frac{8}{7}}\right] ; X=\frac{\alpha_{s}\left(m_{b}\right)}{\alpha_{s}\left(m_{t}\right)} ; Y=\frac{\alpha_{s}\left(m_{t}\right)}{\alpha_{s}\left(M_{W}\right)}
$$

The same calculation holds true for $\Delta m_{s}$, with $B_{s}^{0}$ in place of $B_{d}^{0}$.

\subsubsection{Mixing and $C P$-Violation}

The observation of the decay $K_{L}^{0} \rightarrow \pi \pi$ clearly demonstrates that $C P$ is not an exact symmetry. This is to be expected from the irreducible phase in the CKM matrix. The condition that the matrix be unitary imposes the following relationship ${ }^{8}$ :

$$
V_{u d} V_{u b}^{*}+V_{c d} V_{c b}^{*}+V_{t d} V_{t b}^{*}=0
$$

The relation can be visualized as a triangle in the complex plane, shown in Figure 1.4. The sides are commonly rescaled by $V_{c d} V_{c b}^{*}$. It should be apparent that if the CKM phase $\eta$ were zero, the point $(\rho, \eta)$ would lie on the $x$-axis, and the figure would not be a triangle at all.

The goal of $C P$-violation experiments is to make enough independent measurements of the sides and angles to overdetermine the unitarity triangle, which will then confirm or deny the Standard Model's description. There is wide interest in doing so: for example, cosmological studies [33] of the universe's matter-antimatter asymmetry suggest that additional sources of $C P$-violation are required to explain the observed baryon-to-photon ratio.

\footnotetext{
${ }^{8}$ In fact, there are three independent relationships, but the other two have one term supressed by order $\lambda^{2}$ relative to the other terms, making the verification of the relations difficult in practice.
} 


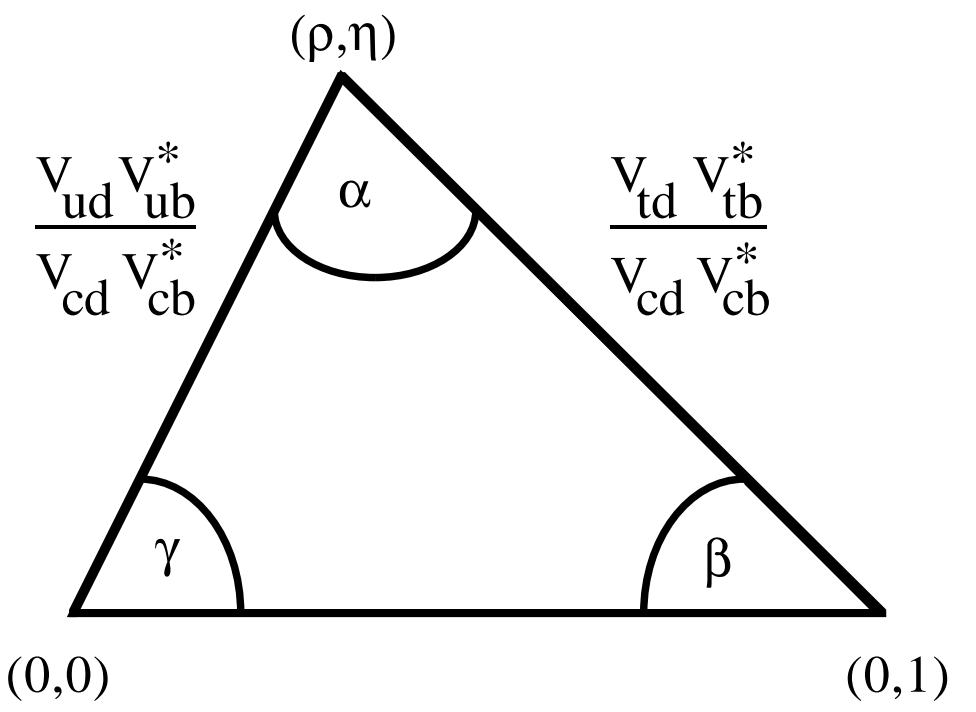

Figure 1.4: A representation of the relation $V_{u d} V_{u b}^{*}+V_{c d} V_{c b}^{*}+V_{t d} V_{t b}^{*}=0$, required by the unitarity of the CKM matrix. The elements form a triangle in the complex plane. Here, the sides have been rescaled by $V_{c d} V_{c b}^{*}$.

A measurement of $B_{d}^{0}$ mixing constitutes part of a measurement of the length of the side $\frac{V_{t d} V_{t b}^{*}}{V_{c d} V_{c b}^{*}}$. In the Wolfenstein parametrization, only $V_{t d}$ is complex. It should be clear from Equation 1.18 that a measurement of $\Delta m_{d}$ can be used to extract $\left|V_{t d}\right|$, as $V_{t b}=1$.

As for the other CKM parameters, $\left|V_{c d}\right|$, the sine of the Cabbibo angle, is quite well-known. $\left|V_{c b}\right|$ can be measured in two ways: first, from the $b$ semileptonic partial width, given by Equation 1.5. This method is less than satisfactory owing to theoretical uncertainties introduced by the imperfectly known $b$ mass and the quark model used to relate the width to the measured $B$ meson semileptonic width. The second method is to measure the rate of the decay $\bar{B} \rightarrow D^{*} \ell \bar{\nu}_{\ell}$ at zero recoil, that can be calculated with little model-dependence. 


\subsubsection{Current $B_{d}^{0}$ Mixing Measurements}

The first convincing observation of $B_{d}^{0}-\bar{B}_{d}^{0}$ mixing was by the ARGUS collaboration in 1987 [34]. In data collected from $e^{+} e^{-}$collisions at the $\Upsilon(4 \mathrm{~S})$, they recorded an event clearly showing the semileptonic decays of two $B_{d}^{0}$ mesons. Additional events, some with a fully-reconstructed hadronic $B_{d}^{0}$ decay opposite a semileptonic decay, and others with two semileptonic decays not reconstructed, also demonstrated that mixing was taking place.

Since that date, $B_{d}^{0}$ mixing has been observed in a number of different collider environments. Measurements at the LEP experiments [35, 36, 37, 38], with data collected from $e^{+} e^{-}$collisions at the $Z^{0}$ resonance, have come to overshadow measurements at the $\Upsilon(4 \mathrm{~S})$, owing to their much larger statistics. $B_{d}^{0}$ mixing has also been observed in $p \bar{p}$ collisions, at the CERN Spp̄S collider [39] and the FNAL Tevatron; this analysis is one such observation. A recent presentation [40] of a preliminary average of all the time-dependent $B_{d}^{0}$ mixing measurements (including this one) gave a value for $\Delta m_{d}$ of $0.460 \pm 0.018 \mathrm{ps}^{-1}$.

One may ask, why measure something which has already been measured? There are three answers to this question: one practical, two philosophical. First, the experimental techniques employed by this analysis can also be employed to study $C P$-violation. The demonstration of a successful $B_{d}^{0}$ mixing measurement serves as a study of CDF's possible sensitivity to $C P$-violation, and hopefully suggests ways in which future detector upgrades can be directed towards improving the sensitivity. Second, a cornerstone of science is that experiments must be repeatable. It is important to perform the same measurements over again to ensure that false results were not obtained. Physics is a human enterprise, and like any such it is prone to human failings - the many false steps along the path of the development of the Standard Model is a testament to this fact. The third answer is related to the 
second. Of the time-dependent $B_{d}^{0}$ mixing measurements, except for those at CDF, all others rely on data collected from $e^{+} e^{-}$collisions. $\Delta m_{d}$ must be measured in as many different ways as possible, to assure that all yield the same value. As this analysis relies on data from $p \bar{p}$ collisions, good agreement between this measurement of $\Delta m_{d}$ and the others will support the validity of the Standard Model.

Every measurement either builds confidence or reveals discrepancies in our understanding of the physical world. It is never a mistake to add new data from old or new environments to the body of knowledge which we possess. This is our duty, as scientists, to science.

\subsection{Overview of the Analysis}

Every mixing measurement is, at its heart, an asymmetry measurement. This is clear from Equations 1.15 and 1.16, as $\Delta m_{d}$ is obtained from the number of mixed and unmixed $B_{d}^{0} \mathrm{~s}$ :

$$
A(t)=\frac{N_{\text {unmixed }}(t)-N_{\text {mixed }}(t)}{N_{\text {unmixed }}(t)+N_{\text {mixed }}(t)}=\cos \left(\Delta m_{d} t\right)
$$

A fit to $A$ verus time will yield $\Delta m_{d}$, the frequency of the cosine. Consequently, one needs four things to perform the measurement:

1. A sample of $B_{d}^{0}$ mesons.

2. The proper time $(\mathrm{c} \tau)$ at which each $B_{d}^{0}$ decayed.

3. Knowledge of whether each decayed as a $B_{d}^{0}$ or a $\bar{B}_{d}^{0}$.

4. Knowledge of whether each was produced as a $B_{d}^{0}$ or a $\bar{B}_{d}^{0}$. 
Regarding the $B_{d}^{0}$ sample, if it is not pure, the proportion of other particles and their effect on items $2-4$ must be known. The time $t$ in Equation 1.21 is the proper time: time as measured in the rest frame of the $B_{d}^{0}$. The data used by this analysis will be the low- $p_{\mathrm{t}}$ inclusive lepton datasets recorded by CDF. These samples are rich in $b \bar{b}$ production by virtue of the direct semileptonic $b$ decay $b \rightarrow c \ell \nu$. The flavor at decay is easily determined, as the lepton $\ell$ must necessarily have the same charge as its parent $b$. The determination of the flavor at production, called flavor-tagging ${ }^{9}$, is more challenging. This analysis exploits the fact that, as $b$ quarks are produced in $b \bar{b}$ pairs, the flavor of one $b$ quark is anticorrelated ${ }^{10}$ with the flavor of the second $b$ quark. Two methods are used to find the second $b$ and tag its flavor: soft lepton tagging, again using semileptonic $b$ decay, and jet charge tagging, which relies on the empirical fact that the average charge of the tracks in a $b$ jet is correlated with the charge, and therefore the flavor, of that $b$.

This dissertation, documenting a time-dependent measurement of $\Delta m_{d}$, is organized as follows: Chapter 2 describes the Tevatron $p \bar{p}$ collider, the CDF detector, and the manner in which the data were recorded. Chapter 3 is concerned with the preparation of samples of high-quality electrons and muons; Chapter 4 with the preparation of final samples by selecting events with displaced vertices associated with the leptons, a signature for $b \bar{b}$ production. Chapter 5 describes the generation of simulated Monte Carlo samples. These allow a number of studies providing insight on the data samples, the most important of which is the calculation of the proper decay time from displaced vertex information. Chapter 6 describes the preparation of samples of fake leptons and the use of them and the Monte Carlo

\footnotetext{
9 "Flavor-tagging" will be used to refer exclusively to the determination of the $b$ production flavor, though of course some would say it more properly means a determination of either the production or the decay flavors.

${ }^{10}$ The anticorrelation is not perfect - it is reduced by backgrounds and the occasions when the second $b$ hadron is a neutral $B$ meson, and has mixed.
} 
samples to determine the proportion of $b \bar{b}, c \bar{c}$, and fake leptons present in the data. The flavor-tagging methods are discussed in Chapter 7, and the determination of $\Delta m_{d}$ is presented in Chapter 8 . 


\section{Chapter 2}

\section{The Tevatron and the CDF Detector}

The Fermi National Accelerator Laboratory (FNAL, or Fermilab) is the nation's premier accelerator facility. More than 2,200 scientists from 36 states and 20 countries use Fermilab's facilities to conduct particle physics research. The jewel of Fermilab's accelerator complex, the Tevatron, is the world's highest-energy particle accelerator.

CDF (the collaboration) came together in 1982 to propose the construction of a general-purpose detector to study the physics of $p \bar{p}$ collisions at Fermilab. CDF (the detector) recorded its first data in 1987 and recently concluded a data-taking Run in 1996. The detector was designed to be sensitive to a wide range of high- $p_{\mathrm{t}}$ physics, studying the $W$ and $Z^{0}$ bosons, $b$ hadrons, and searches for the top quark, Higgs boson(s), and numerous exotic particles (supersymmetric partners and leptoquarks, to name two). In 1995 this promise was kept by the joint discovery of the top quark with D0, CDF's companion collider experiment.

The CDF detector [41] consists of tracking, calorimetry, and muon subsystems arranged in a forward-backward and azimuthally-symmmetric geometry. The tracking chambers detect the passage of charged particles and lie inside an axial magnetic 
field, which curves the trajectories of charged particles in helices; the radius of curvature constitutes a measurement of their transverse momenta. Outside the tracking chambers lie the calorimeters, which provide a direct energy measurement of both hadronic jets and "electromagnetic" photons and electrons. Furthest out are the muon chambers, located behind steel shielding which only minimum-ionizing muons are likely to reach. There are currently over 150,000 electronic readout channels total from all the subdetectors.

CDF has had a long and rich history and, over the years, many of the detectors have been improved or replaced altogether. Time at CDF is measured in "Runs", those periods ${ }^{1}$ when the accelerators are operated in colliding-beam mode to provide data to CDF, D0, and other smaller experiments. The most recent as of this writing, Run 1, was divided into three portions: 1A, 1B, and 1C. Run 1A and Run 1B are usually referenced in publications by their dates, as the 1993-1994 Run and 19941995 Run (respectively), though the former usage will be used here. A number of detectors which will be described were installed for the commencement of Run 1A, and one, SVX', was first used in Run 1B. Run 2 is scheduled to begin in 1999; many major detector upgrades [42] are planned to meet the challenge presented by improvements to the accelerators. And yet it is a testament to the efforts of many physicists and engineers that many original detector components first used in 1987 are not only a critical component of this analysis, a decade later, but will continue to be an integral part of $p \bar{p}$ collider research in the next century.

\footnotetext{
${ }^{1}$ Another unit of time is the "run", a period of contiguous data-taking. A Run may be measured in several months or years; a run in several hours. This capitalization will be used consistently to avoid any confusion.
} 


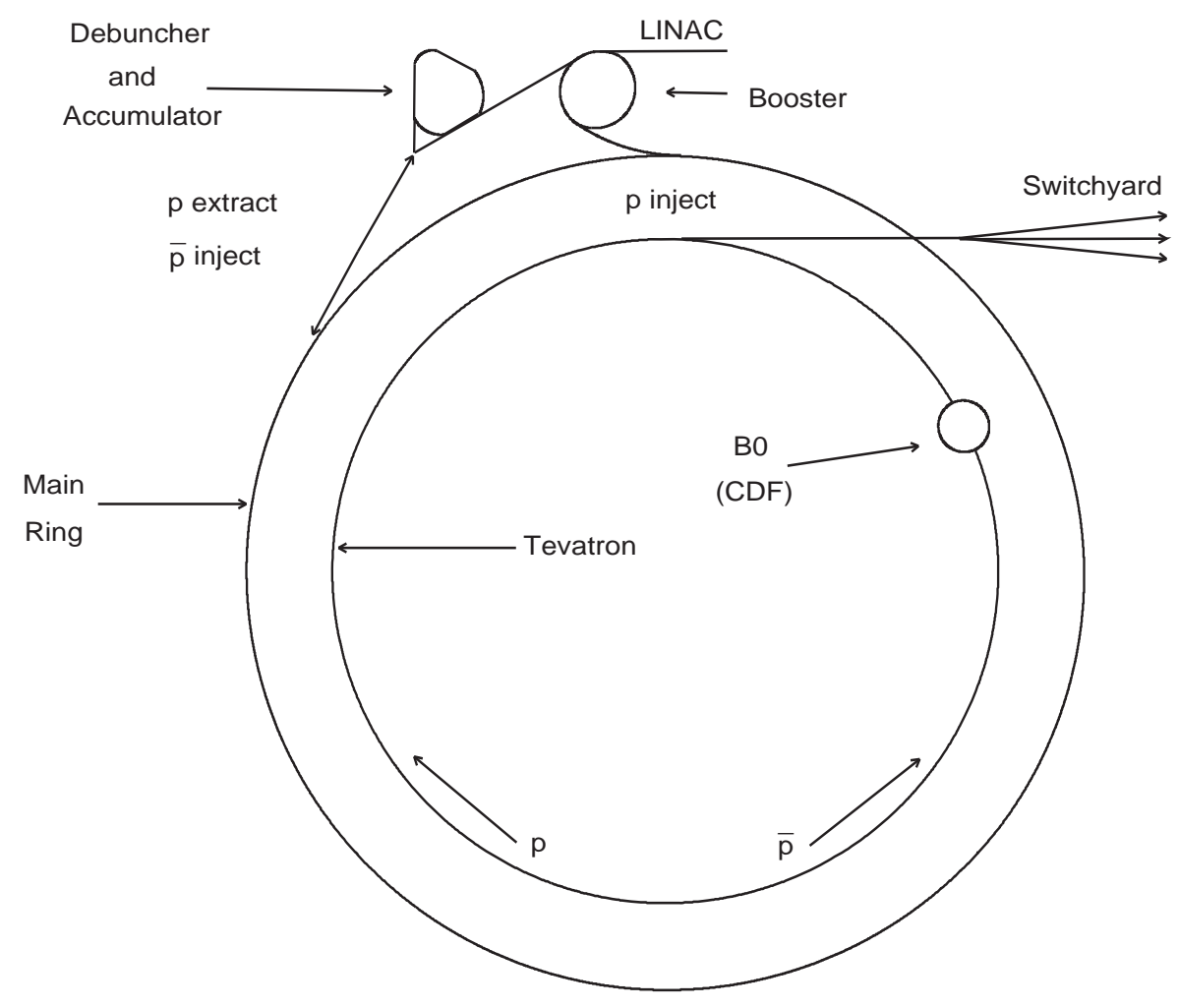

Figure 2.1: Schematic representation of the Tevatron and supporting particle accelerators. The $\mathrm{B} 0$ interaction region (the location of $\mathrm{CDF}$ ) is also indicated.

\subsection{Particle Accelerators at FNAL}

There are a number of different particle accelerators and beamlines at Fermilab, each serving the research needs of one or more experiments. Only the elements of the $p \bar{p}$ collider system will be described here. Figure 2.1 shows a schematic representation of the collider layout.

The process of producing $p \bar{p}$ collisions begins by ionizing hydrogen gas and accelerating the $\mathrm{H}^{-}$ions to $750 \mathrm{KeV}$ with a Cockcroft-Walton electrostatic accelerator. The ions are transferred to a linear accelerator (Linac), where their energy is increased to $200 \mathrm{MeV}$. The electrons are then stripped from the $\mathrm{H}^{-}$ions to leave 
only protons, which are transferred to the Booster Ring, a small (by today's stan-

dards) synchrotron, where they are accelerated to $8 \mathrm{GeV}$, collected into bunches, and stored. The proton bunches are injected into the Main Ring, a second synchrotron, which can accelerate them to a per-proton energy of $150 \mathrm{GeV}$.

During $p \bar{p}$ collider operations, protons are extracted from the Main Ring and directed onto a beryllium target, producing a collection of high-energy interaction products. Antiprotons are selected from the mix by a mass spectrometer, cooled, then stored in the Accumulator Ring. When sufficient numbers of antiprotons have been collected, they are injected into the Main Ring. As antiprotons have the opposite charge of protons, they can be accelerated in the same synchrotrons as protons, travelling in the opposite direction, using electrostatic separators to prevent them from interacting except where desired.

Bunches of protons and antiprotons are transferred from the Main Ring to the Tevatron, a synchrotron constructed of superconducting magnets, which lies just below the Main Ring in the same tunnel. The Tevatron can hold up to six bunches of each at a time. After it is filled, the Tevatron ramps the bunches to its full energy of $900 \mathrm{GeV}$ and proceeds to collide them at a number of interaction regions around the ring. When the number of antiprotons falls too low, the beam is dumped, and new bunches are injected.

\subsubsection{The B0 Interaction Region}

$\mathrm{B} 0$ is the name given to the $p \bar{p}$ interaction region which the CDF detector surrounds (see Figure 2.1). The aptly named D0 detector is at the D0 interaction region; A0 houses the abort kicker, specialized magnets which can quickly (between bunch crossings) eject the beam from the Tevatron. The proton and antiproton beams are brought into collision at B0 by strong-focussing quadrupole magnets. The intensity 
of the colliding beams is measured in instantaneous luminosity, which has units of inverse-area-squared per second. The likelihood of particle interactions (the crosssection) is measured in area-squared; intuitively, the probability that two objects will collide is proportional to the area that they overlap. A convenient unit for cross-sections is the barn: $1 \mathrm{~b}=10^{-24} \mathrm{~cm}^{2}$.

The number of interactions per second at CDF is the total $p \bar{p}$ interaction crosssection times the instantaneous luminosity provided by the Tevatron. The latter can be calculated explicitly:

$$
\mathcal{L}=\frac{N_{p} N_{\bar{p}} f}{4 \pi \sigma_{x} \sigma_{y}}
$$

where $N_{p}$ and $N_{\bar{p}}$ are the number of protons and antiprotons per bunch, $f$ is the collision frequency, and $\sigma_{x}$ and $\sigma_{y}$ are the major and minor axis of the elliptical cross section of the beam profile at the interaction point.

In a typical store, six bunches of $18 \times 10^{10}$ protons are directed onto six bunches of $6 \times 10^{10}$ antiprotons with a bunch-crossing time of $3.5 \mu \mathrm{s}$. The magnets which bring the beams into collision compress the transverse extent of the bunches into a circular shape, with an approximate radius of $\sigma=35 \mu \mathrm{m}$. According to Equation 2.1, then, the typical instantaneous luminosity supplied to CDF is approximately $15 \times 10^{30} \mathrm{~cm}^{-2} \mathrm{~s}^{-1}$. Of course, as a store progresses, the number of bunch particles falls from interactions or beam losses, and the instantaneous luminosity decays. Data during Run 1B were taken mostly over a range of $5-20 \times 10^{30} \mathrm{~cm}^{-2} \mathrm{~s}^{-1}$.

High luminosity presents many experimental challenges. Detectors and/or the data acquisition system may be overloaded by the high frequency of interactions; multiple $p \bar{p}$ collisions in a single bunch crossing may yield particles in the detector which come from very different physics processes. The former was addressed during Run $1 \mathrm{~B}$ by the adoption of dynamic trigger prescaling, limiting the rate at which data is recorded, emphasizing certain physics processes over others, thus enabling 
the experiment to meet its research goals. The latter is a concern of this analysis; a number of studies will be presented showing that there is no detectable adverse effect from high instantaneous luminosity.

\subsection{The CDF Detector}

As previously mentioned, the CDF detector [41] is a multipurpose detector, consisting of tracking, calorimetry, and muon subsystems. The readout of the detectors is controlled by the trigger, hardware and software which examines the data for particular physics processes and acts as a "gatekeeper", recording on magnetic tapes only those collisions (called events) which are considered "interesting". Further data processing is later performed on recorded data, which will be discussed in the next chapter.

Tracking is restricted to the central part of the detector. The CDF tracking system achieves excellent position and momentum resolution due to a high axial magnetic field of 1.41 Tesla and large measurement volume. The calorimetry systems consist of projective towers of alternating absorber and scintillator which point back to the nominal interaction vertex for energy measurements of both electromagnetic and hadronic showers. The muon system consists of drift chambers to record position information for charged particles which are not absorbed in the calorimeters.

The CDF coordinate system has the $z$-axis along the proton beam, the $x$-axis pointing away from the center of the Tevatron, and the $y$-axis pointing up. Figure 2.2 shows a representation of the CDF detector and its coordinate system. As CDF is roughly cylindrically-symmetric, cylindrical coordinates are often more use-

ful. Thus, in this coordinate system, $\phi$ is the azimuthal angle, $\theta$ is the polar angle 
from the $z$ axis, and $r$ is the radius to the $z$ axis. A useful geometric parameter is the pseudorapidity, defined as $\eta=-\ln [\tan (\theta / 2)]$, as particle production is roughly uniform in $\eta$.

Figure 2.3 is another representation of the CDF detector giving a threedimensional perspective view.

\subsubsection{Tracking}

The Run 1B tracking system consists of four elements: the solenoid, the vertex time projection chamber (VTX), the central tracking chamber (CTC), and the silicon vertex detector $\left(\mathrm{SVX}^{\prime}\right)$. Each of the detectors measures the position of charged particles by collecting the charge produced by their ionization in the detectors' material. The tracking chambers work in concert: "hits" left by the passage of particles are grouped by pattern recognition software into track segments; segments in the CTC and VTX are matched to each other, and in turn, matched to hits in SVX'. The solenoid completely surrounds the three tracking chambers, providing an axial magnetic field in which the trajectories of charged particles are curved into helicies.

A helix is described by five parameters: $\cot \boldsymbol{\theta}$, the tilt angle of the helix; $C$ (the curvature), the inverse of the diameter of the inscribed circle defined by the projection of the helical path into the $x-y$ plane; $\boldsymbol{z}_{\mathbf{0}}$, the $z$ position; $\boldsymbol{D}_{\mathbf{0}}$ (the impact parameter), the perpendicular distance to the origin; and $\phi_{0}$, the azimuthal direction. All quantities are measured at the position of closest approach to the origin. The impact parameter is a signed quantity: it is positive for positivelycharged tracks whose inscribed circle encloses the origin and for negatively-charged tracks whose circle excludes the origin. The curvature is also signed, but by the charge of the particle. Coupled with knowledge of the magnetic field strength, it 


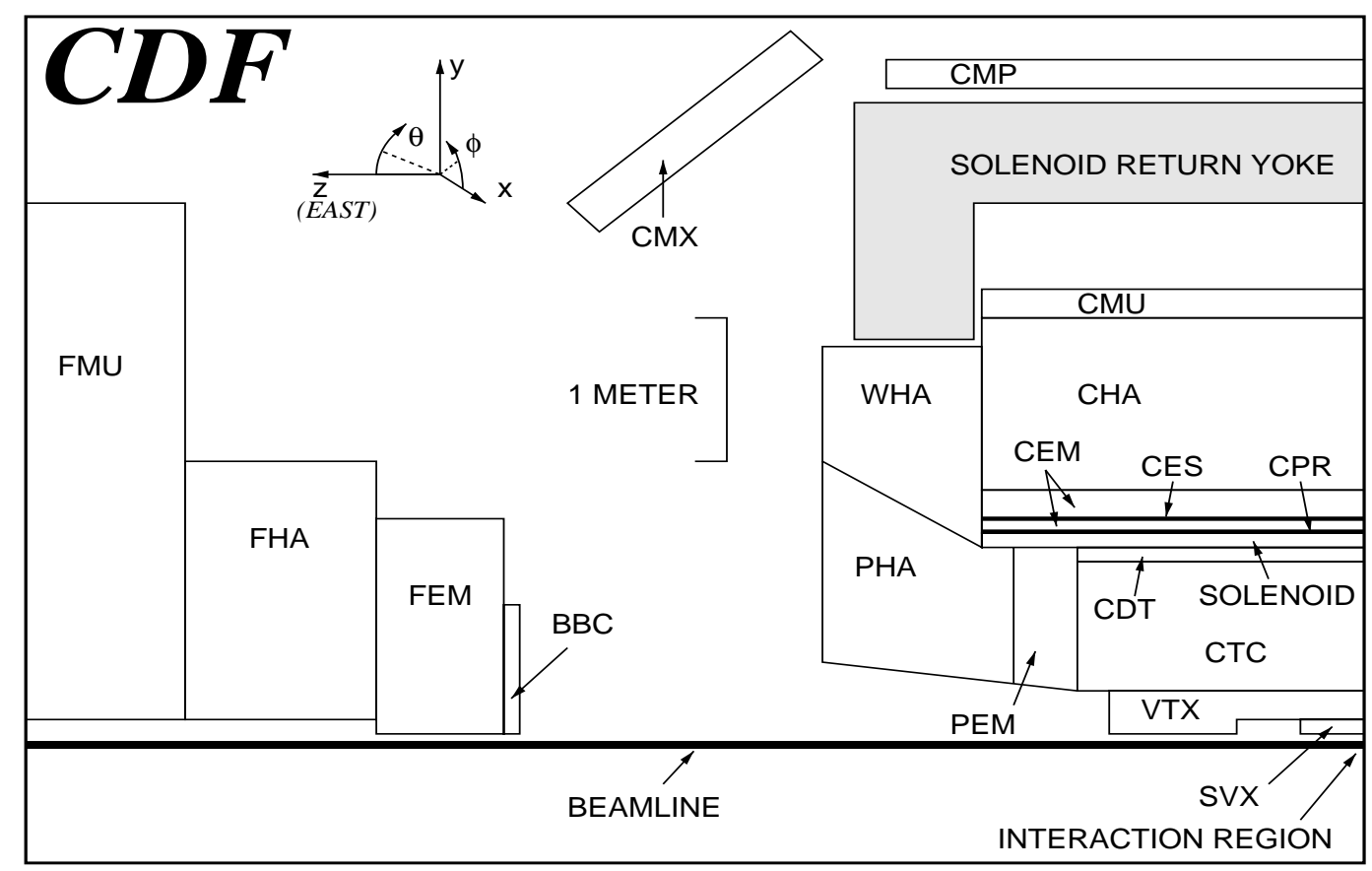

Figure 2.2: The Run 1 CDF detector shown as one-quarter of an elevation view. The other quarters look the same, as the detector is forward-backward and azimuthally symmetric.

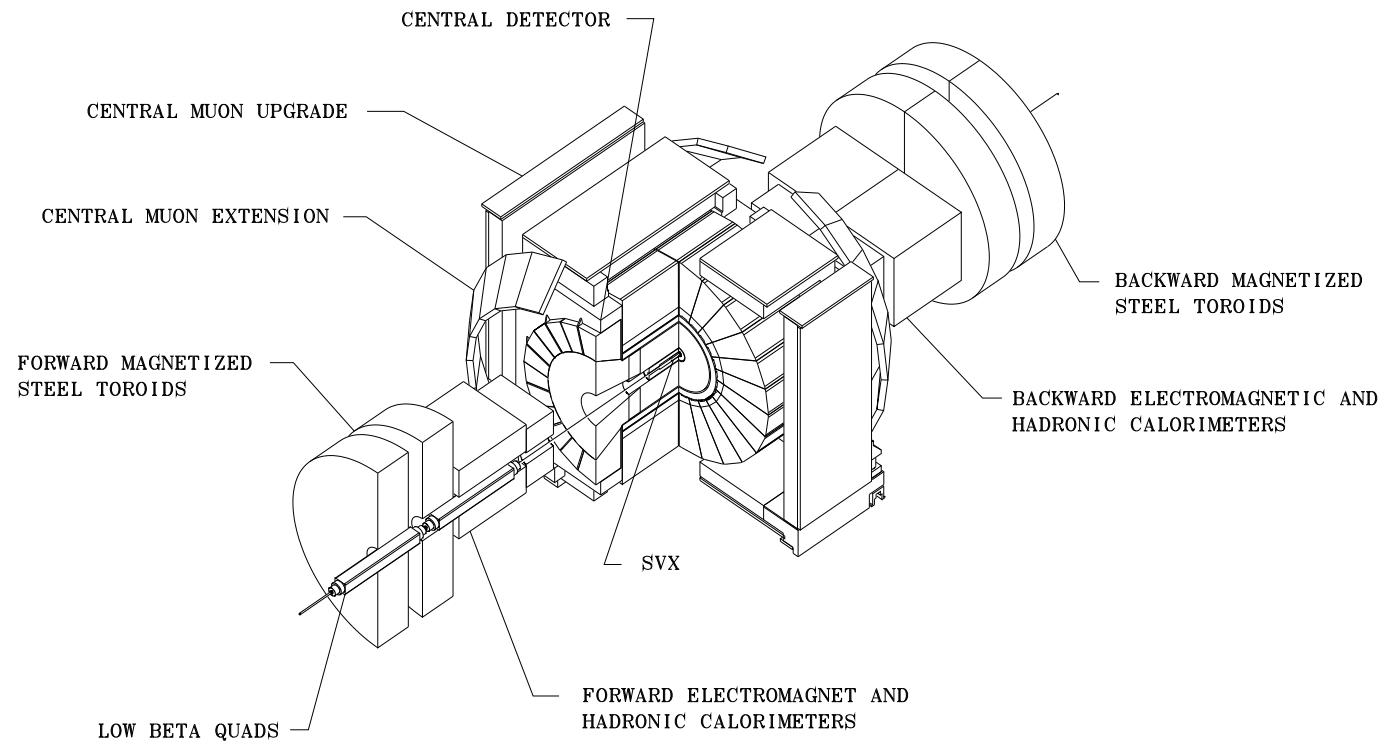

Figure 2.3: Perspective view schematic of the Run 1 CDF detector. 
gives a determination of the transverse momentum. The absence of a track can also be a powerful measurement; neutral particles, such as photons or $\pi^{0} \mathrm{~s}$, do not leave an ionization trail in the tracking chambers, but do deposit their energy in the calorimeters.

\section{Solenoid}

The CDF solenoid coil [43] is a superconducting magnet, 3 meters in diameter and 4.5 meters long, designed to enfold the tracking chambers in a uniform 1.41 Tesla magnetic field. The magnetic field flux outside the coil is returned through a steel yoke, to avoid having the fields interfere with the proper operation of the calorimeters; the yoke also serves as the calorimeters' mechanical support. The small nonuniformities of the magnetic field have been mapped [44], allowing for appropriate momentum corrections for tracks found by the pattern recognition.

\section{CTC}

The CTC [45] is a cylindrical wire tracking chamber which covers $|\eta|<1.1$. It is the largest of the tracking chambers, occupying most of the interior of the solenoid. Table 2.1 lists some of the CTC's mechanical and operational parameters.

There are 84 sense wire layers arranged in five axial (12 wires per) and four stereo (6 wires per) superlayers. The axial superlayers have their wires strung parallel to the $p \bar{p}$ beam axis; the stereo superlayers' wires are strung with an alternating $\pm 3^{\circ}$ tilt relative to that axis. This construction allows a measurement of the $z$ position of traversing particles. Within each superlayer, the cells of sense wires and fieldshaping wires have a $45^{\circ}$ tilt with respect to the radial direction, matching the Lorentz angle $^{2}$. The large tilt also ensures there is a large overlap between adjacent

\footnotetext{
${ }^{2}$ The ionization left by a particle's passage is collected on the sense wires. As this charge moves
} 
cells, such that a high- $p_{\mathrm{t}}$ track must pass close to at least one sense wire in every superlayer. A diagram of the CTC endplate, the support structure for the CTC wires, is shown in Figure 2.4, illustrating the geometry of the superlayers and the tilt of the cells.

The electronics that read out the CTC record the amount of charge collected on each wire by measuring the width of the current pulse. A sufficiently long pulse width indicates the passage of a particle. The pulse width can also be used to measure the ionization energy loss $(d E / d x)$ of the particle in the CTC gas $^{3}$. Determining both momentum and $d E / d x$ corresponds to a mass measurement, which allows discrimination between different particle species. The CTC $d E / d x$ system is used in this analysis to either select or reject electrons. It is described in greater detail in Appendix C.

Tracks are reconstructed in the CTC by first assembling track segments in the cells of each superlayer. Each segment has a left-right symmetry, that is, a particle's ionization trail, collected on the sense wires, may lie to the left or right of the wire plane. The "confusion" is removed by connecting segments in adjoining superlayers into a single trajectory; this is possible with only one choice of left or right for each segment. A reconstructed track can be assured of being well-measured by requiring a minimum number of hits in a minimum number of superlayers. The phrase "standard track quality cuts" should be taken to refer to the requirement of at least four or more hits in two or more axial superlayers and two or more hits in two or more stereo superlayers. The separate requirements for two of each type of superlayer assures that the track is well-measured in $z$ as well as the $x-y$

in both electric and magnetic fields, the drift direction is at an angle to the electric field. Given that the strength of the magnetic field is selected to ensure good momentum resolution and that of the electric field to saturate the drift velocity, a large Lorentz angle is convenient. The $45^{\circ}$ tilt of the cells matches the Lorentz angle such that the drift direction is approximately azimuthal.

${ }^{3}$ The CTC gas is a mixture of argon, ethane, and ethanol, in the ratio $49.6 \%: 49.6 \%: 0.8 \%$. 
plane; this can be confirmed by a requirement that the segments make a successful three-dimensional helical fit.

\section{VTX}

The VTX, just inside the CTC and surrounding SVX', consists of 56 octagonal modules with sense wires strung in the $r$ - $\phi$ plane. The VTX wires are perpendicular to the CTC wires, with the drift direction in the $z$ plane, allowing $r$ - $z$ measurements of tracks' positions with a Lorentz angle of zero. The 36 modules around SVX' contain 16 sense wires in each octant; the 20 modules at larger $z$ have 24 wires, allowing measurements closer to the beamline for those tracks not in the fiducial volume of SVX'.

The VTX has the best $z$ resolution of any of CDF's tracking chambers, though its resolution is comparatively worse in the other coordinates. Adjacent VTX modules are rotated by $11.3^{\circ}$ in $\phi$ so CTC track segments (with their lesser $z$ resolution) can be matched to VTX track segments without confusion. The main use of the VTX is to determine the $z$ position of the $p \bar{p}$ collisions, which, when coupled to the $x-y$ position of the $p \bar{p}$ beamline determined by the CTC and SVX', gives the best measurement of the three-dimensional position of the primary interaction vertex. This is discussed in greater detail in Section 4.2.

\section{$\mathrm{SVX}^{\prime}$}

SVX' [46], a silicon microstrip detector, is the innermost tracking detector, closest to the beampipe. Its cylindrical configuration and fine segmentation allows precision measurements in the $r-\phi$ plane to best determine the impact parameter of charged particles. The knowledge of the curvature and $\phi$ of tracks is also greatly improved by its use, though it offers essentially no information about $z$ or $\cot \theta$. 


\begin{tabular}{|ll|}
\hline Number of layers & 84 \\
Number of superlayers & 9 \\
Stereo angle for each superlayer & $0^{\circ},+3^{\circ}, 0^{\circ},-3^{\circ}, 0^{\circ},+3^{\circ}, 0^{\circ},-3^{\circ}, 0^{\circ}$ \\
Number of cells per superlayer & $30,42,48,60,72,84,96,108,120$ \\
Number of sense wires per cell & $12,6,12,6,12,6,12,6,12$ \\
Sense wire spacing & $0.1 \mathrm{~cm}$ \\
Tilt angle & $45^{\circ}$ \\
Radius at innermost sense wire & $30.9 \mathrm{~cm}$ \\
Radius at outermost sense wire & $132.0 \mathrm{~cm}$ \\
Wire length & $321.4 \mathrm{~cm}$ \\
Electric Field & $1350 \mathrm{~V} / \mathrm{cm}$ \\
Magnetic Field & $1.41 \mathrm{~T}$ \\
\hline Nominal $r-\phi \mathrm{spatial}$ resolution & $200 \mu \mathrm{m}$ \\
Nominal $r-z$ spatial resolution & $0.6 \mathrm{~cm}$ \\
Nominal $\Delta p_{\mathrm{t}} / p_{\mathrm{t}}$ & $0.002 \times p_{\mathrm{t}}(\mathrm{GeV} / \mathrm{c})$ \\
Nominal $\Delta p_{\mathrm{t}} / p_{\mathrm{t}}$ (beam constrained $)$ & $0.0011 \times p_{\mathrm{t}}(\mathrm{GeV} / \mathrm{c})$ \\
\hline
\end{tabular}

Table 2.1: CTC mechanical and operational parameters.

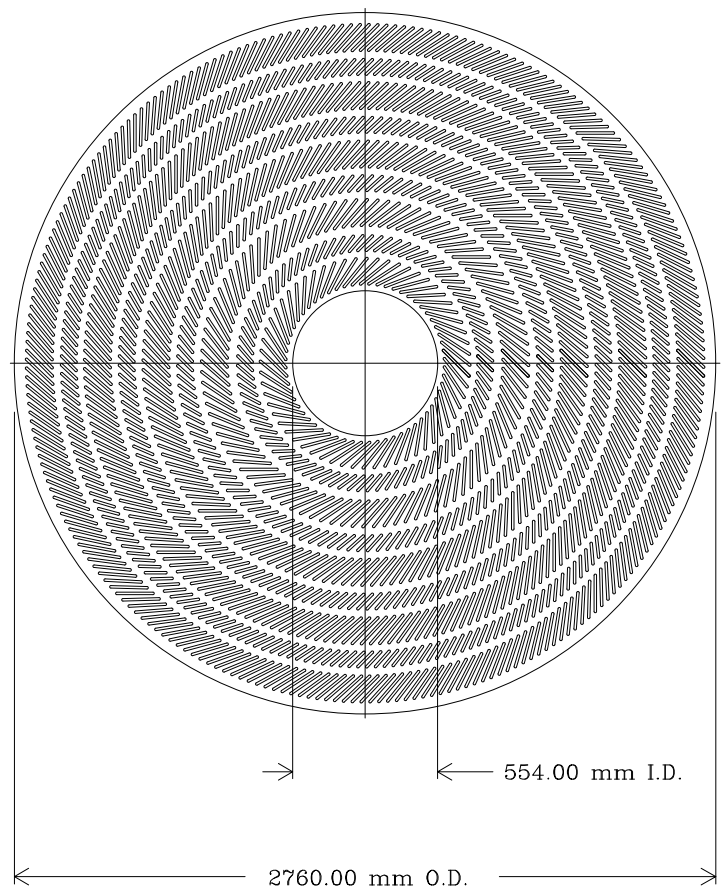

Figure 2.4: Schematic of the CTC endplate showing the superlayer structure and $45^{\circ}$ tilt of the wire cells. 
CDF's first silicon vertex detector, the SVX [47], was installed before the commencement of Run 1A. The SVX served the experiment well, but sustained steady radiation damage (total dose: $\sim 15 \mathrm{kRad}$ ) over the course of the Run. It was known well in advance that a replacement would be needed to withstand the projected $60 \mathrm{kRad}$ dose from the longer running time of Run 1B. The new detector, $\mathrm{SVX}^{\prime}$, is an improved version of the SVX. The readout chips were fabricated with a radiation-hardened process and designed to provide a higher signal-to-noise for improved tracking performance. The silicon strips were AC-coupled to the chips to immunize them from increased leakage current due to bulk radiation damage. The position of the innermost silicon detectors was changed to remove the $\phi$ gaps that were part of the original SVX mechanical design. Otherwise, the two detectors are remarkably similar, and much of the information here applies equally to both. A comparison of the two detectors is made in Table 2.2. In chapters subsequent to this one, any reference to "SVX" should be understood as referring to the SVX' detector; only Run 1B data is used in this analysis.

SVX' consists of 46,080 silicon microstrip detectors, arranged in a cylindrical configuration around the beampipe. It is divided into two barrels, with the division at $z=0$. Each barrel is composed of twelve wedges, each subtending $30^{\circ}$; each wedge has four ladders, placed at various radii. The ladders are held by a bulkhead at the ends of each barrel; the bulkhead is made of beryllium to minimize the probability of an interaction with particles from $p \bar{p}$ collisions. An $\mathrm{SVX}^{\prime}$ barrel is shown schematically in Figure 2.5, showing the wedge-ladder configuration. Ladders at a given radius are referred to both individually and collectively as a layer. The ladders in Layer 0 were given an extra tilt of $1^{\circ}$ and moved closer to the beampipe compared to the SVX detector. The tilt allows adjacent ladders to overlap, and the smaller radius a better measurement of the impact parameter. 
A ladder is the basic detector unit, shown schematically in Figure 2.6. It consists of three $8.5 \mathrm{~cm}$ long silicon crystals with the readout electronics on one end, all mounted on a carbon-fiber-reinforced foam support. The crystals have been implanted with microstrips at a $60 \mu \mathrm{m}$ pitch (55 $\mu \mathrm{m}$ for Layer 3 crystals). The strips on one crystal are joined by microbonds to the strips of the next, making a total active length of $25.5 \mathrm{~cm}$. The strips are $\mathrm{AC}$ coupled to the readout chips, and the bulk silicon of the detectors is biased by a single-sided FOXFET. "Bad" strips, those with excessive noise or a damaged FOXFET connection, are either disconnected from the chips or shorted to ground as appropriate. $1.73 \%$ of the strips in $\mathrm{SVX}^{\prime}$ are bad.

Particles traversing the voltage-biased silicon leave an ionization trail; the charge is collected on the strips and integrated by the readout chips. The AC coupling of SVX $^{\prime}$ allows the operation of the readout in double sample-and-hold mode, with one charge integration, whereas SVX required quadruple sampling, with two integrations, one subtracted from the other. This difference alone is responsible for a noise reduction by a factor of $\sqrt{2}$. The readout chips are also capable of sparse readout - only those strips with a collected charge above a specified threshold are read, resulting in a significant reduction in readout time. "Neighbor logic" is also available, allowing the readout of the strips immediately next to a strip with charge above threshold, even if they themselves are not. SVX' is typically operated in sparse mode, with the neighbor logic activated.

Unlike the other tracking chambers, where charge collected on a single wire serves as a measurement of a particle's trajectory, $\mathrm{SVX}^{\prime}$ defines hits not by individual strips, but rather as clusters of strips. Clusters, constructed by offline software, are defined as contiguous strips whose charge pulseheights are each greater than a factor $M$ times their noise, as some strips are noisier than others. Clusters with more 
strips are allowed to have a lower threshold on each strip: $M=4.0,2.5$, and 2.0 for one-strip, two-strip, and larger clusters, respectively. The pulseheight-weighted position average, coupled with the known layer radii, constitutes the measurement of a traversing particle's $r-\phi$ location. Tracks in the CTC are matched to clusters in $\mathrm{SVX}^{\prime}$ through road-search pattern recognition, where the track is projected backwards from the CTC to the interaction vertex, and a region (commensurate with the size of the error on the projected location at a given layer) searched for acceptable clusters. Layer 3, the outermost, is the first layer searched. If a cluster is matched to a track, it is included in the fit of the track's path; understandably, the road narrows as successive clusters are included.

The correct alignment of SVX', both internally (ladder-to-ladder) and externally (to the CTC), is of critical importance to this procedure. An initial estimate of the external alignment was obtained during installation; the internal alignment was measured to $\sim 19 \mu \mathrm{m}$ during construction. Both were improved iteratively using collected data, resulting in a position resolution of $9.7 \mu \mathrm{m}$. Including the contribution from multiple scattering in the beampipe yields an impact parameter resolution of $11.3 \mu \mathrm{m}$ in the high- $p_{\mathrm{t}}$ limit. This analysis makes good use of this excellent resolution.

\subsubsection{Calorimetry}

Calorimeters (as the name indicates) provide a direct energy measurement of incident particles. This is accomplished by alternating layers of dense material with detector elements; particles interact with the material and shower, that is, produce numerous secondary particles of lower energy. The total number of particles in the shower is a measurement of the energy of the incident particle (given a thick enough "sandwich" of absorber/detector to contain the entire shower). To take 


\begin{tabular}{|c|c|c|}
\hline Feature & SVX & $\mathrm{SVX}^{\prime}$ \\
\hline channels & \multicolumn{2}{|c|}{46080} \\
\hline$z$ coverage & \multicolumn{2}{|c|}{$51.1 \mathrm{~cm}$} \\
\hline gap at $z=0$ & \multicolumn{2}{|c|}{$2.15 \mathrm{~cm}$} \\
\hline radius L0 & $3.0049 \mathrm{~cm}$ & $2.8612 \mathrm{~cm}$ \\
\hline radius L1 & \multicolumn{2}{|c|}{$4.2560 \mathrm{~cm}$} \\
\hline radius L2 & \multicolumn{2}{|c|}{$5.6872 \mathrm{~cm}$} \\
\hline radius L3 & \multicolumn{2}{|c|}{$7.8658 \mathrm{~cm}$} \\
\hline overlap L0 & \multicolumn{2}{|c|}{$-1.26 \mathrm{deg}$ (gap) $0.17 \mathrm{deg}$ (.24 strip) } \\
\hline overlap L1 & \multicolumn{2}{|c|}{$0.32 \mathrm{deg}(4 \mathrm{strip})$} \\
\hline overlap L2 & \multicolumn{2}{|c|}{$0.20 \mathrm{deg}$ ( 4 strip) } \\
\hline overlap L3 & \multicolumn{2}{|c|}{$0.04 \mathrm{deg}$ (0 strip) } \\
\hline silicon & \multicolumn{2}{|c|}{ one-sided } \\
\hline & $\mathrm{DC}$ & AC FOXFET bias \\
\hline passivation & none & silox \\
\hline atmosphere & $\mathrm{Ar} /$ Ethane $+\mathrm{H}_{2} \mathrm{O}$ & dry nitrogen \\
\hline $\begin{array}{l}\text { readout chip } \\
\text { sampling }\end{array}$ & $\begin{array}{l}\text { SVX IC Rev.D } \\
\text { quadruple }\end{array}$ & $\begin{array}{l}\text { SVX IC Rev.H3 } \\
\text { double }\end{array}$ \\
\hline noise & 2200 electrons & 1300 electrons \\
\hline gain & $15 \mathrm{mV} / \mathrm{fC}$ & $21 \mathrm{mV} / \mathrm{fC}$ \\
\hline reset/integrate & \multicolumn{2}{|c|}{$2.5 \mu \mathrm{s}$} \\
\hline readout time & $2.7 \mu \mathrm{s}$ & $2.1 \mu \mathrm{s}$ \\
\hline rad. dose limit & $\sim 20 \mathrm{kRad}$ & $>1 \mathrm{MRad}$ \\
\hline bad channels & $1.59 \%$ & $1.73 \%$ \\
\hline occupancy (typical) & $7 \%-10 \%$ & $5 \%$ \\
\hline occupancy (max) & $12 \%-20 \%$ & $25 \%$ \\
\hline
\end{tabular}

Table 2.2: Comparison of SVX and SVX' mechanical and operational parameters. 


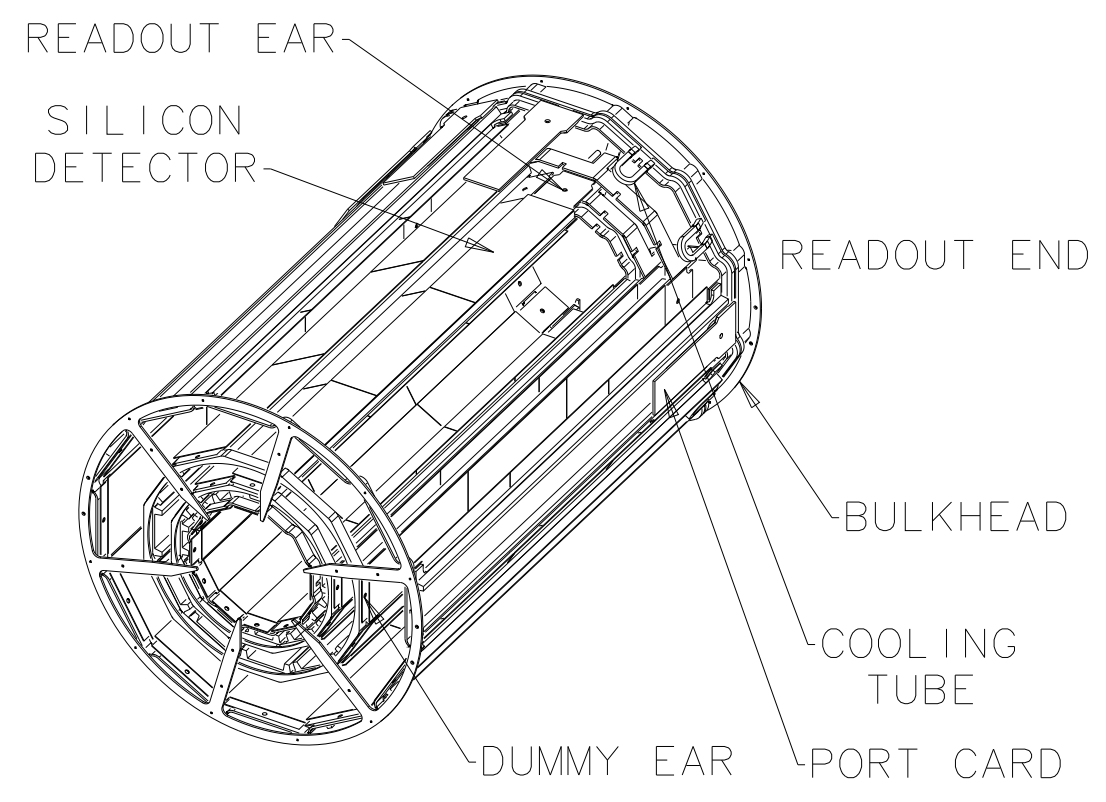

Figure 2.5: Schematic representation of an $\mathrm{SVX}^{\prime}$ barrel.

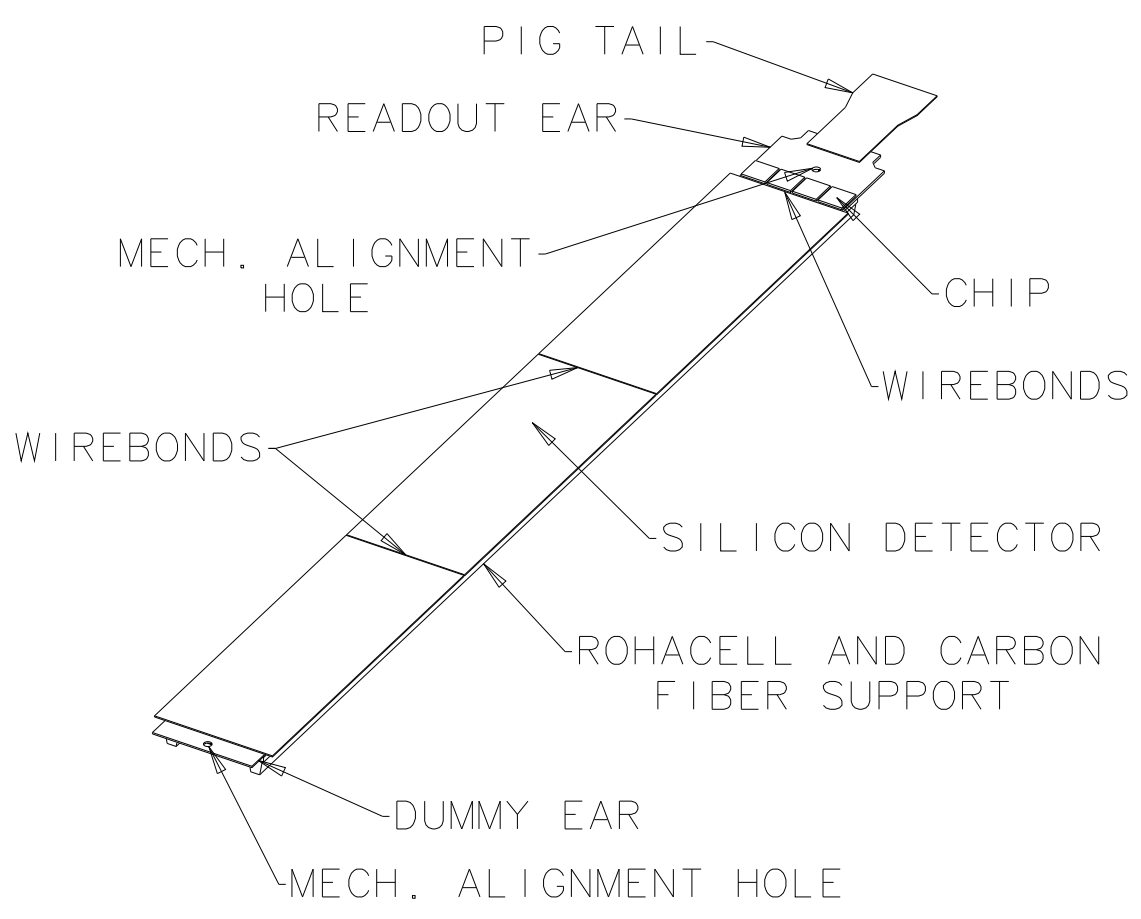

Figure 2.6: Schematic representation of an $\mathrm{SVX}^{\prime}$ ladder. 
advantage of the ways in which different types of particles interact with matter, the CDF calorimetery system consists of many paired subsystems, each with an electromagnetic calorimeter, located closest to the interaction point, and a hadronic calorimeter, much thicker, with many more absorber/detector layers. The absorber is lead in all the electromagnetic calorimeters and steel in all the hadronic calorimeters.

Charged particles traversing the material of the detector will lose energy through electromagnetic interactions; they may ionize atoms in the material or radiate photons when accelerated in the electric field of atomic nuclei (bremsstrahlung). Radiated photons will convert to electron-positron pairs, which in turn lose energy in the detector. For particles with sufficient energy (only $6.9 \mathrm{MeV}$ for electrons in lead), radiation losses dominate. The range of incident particles through matter owing to bremsstrahlung is proportional to the square of the mass of the particle; thus electrons and photons will have a much shorter range in dense material than will more massive particles. CDF's electromagnetic calorimeters are designed to contain the showers from electrons and photons, though heavier particles typically pass through.

Hadrons may also lose energy though strong inelastic collisions with atomic nuclei. As the nuclear absorption length is much shorter for hadrons than the radiation length, energy loss is dominated by secondary hadron production. The hadron calorimeters provide many layers of steel (4.7 absorption lengths), complementing the lead in the electromagnetic calorimeters (1 absorption length), in order to contain hadronic showers. Muons, which do not interact strongly, will penetrate both calorimeters if sufficiently energetic.

Different regions of pseudorapidity are covered by different calorimeters; each 
of the subsystems are essentially rotationally symmetric (in $\phi)$. The central electromagnetic and hadronic calorimeters (CEM and CHA) cover $|\eta|$ up to 0.8, while the endwall hadronic calorimeter (WHA) covers $|\eta|$ of 0.6 to 1.3. The CEM, CHA, and WHA alternate layers of scintillator with layers of absorber. The plug (PEM and PHA) and forward calorimeters (FEM and FHA) are gas calorimeters which provide coverage in $|\eta|$ between 1.3 and 2.4 and 2.4 to approximately 4 , respectively. Figures 2.2 and 2.3 show graphically the locations of the various calorimeters. Each subsystem is segmented into wedges, subtending some angle in $\phi$; each wedge is segmented into projective towers. Each tower therefore subtends some portion of $\eta$ - $\phi$ space, angled to point back to the CDF origin. A schematic of a central calorimeter wedge is shown in Figure 2.7, showing the light-pipe configuration used to read out the scintillators.

Calorimeter jets are defined by searching for clusters of energy deposited in nearby calorimeter towers [48]. The procedure begins by identifying single towers above a standard energy threshold, then adding the energy of its neighbors to the total. Three standard clustering sizes are used at CDF, specified by their maximum extent in $\Delta R$, where $(\Delta R)^{2}=(\Delta \eta)^{2}+(\Delta \phi)^{2} . \Delta R$ defines a cone in $\eta$ - $\phi$ coordinates, which is projected to the face of the calorimeters to trace a region of includable towers. The three standard cone sizes are $\Delta R=0.4,0.7$, and 1.0. Only those calorimeter jets found with an 0.7 cone size play a role in this analysis.

The calorimeter information is used by this analysis in a number of ways. The tower traversed by muon candidates is checked to ensure that it has sufficient deposited energy to be consistent with the passage of a minimum-ionizing particle. Jets serve to collect and organize SVX tracks for a $b$ jet-tagger (see Section 4.3). But the most important use of the calorimeter is the identification of electron candidates. They are selected by the trigger based upon clusters of electromagnetic energy in 
the CEM; calorimeter measurements are then subsequently used to ensure their quality.

The success of the energy clustering and triggering depends on the physical separation of the electron from other particles. The signature of an electron is a cluster of mostly electromagnetic energy with a single track pointing to the cluster; hadrons sufficiently close to the electron will deposit their energy in the same towers, but in the hadronic calorimeters, masking the electron signature. This implicit isolation requirement of the trigger is actually beneficial to this analysis: electrons from semileptonic $b$ decay are much more likely to be isolated than those from other processes, owing to the large $b$ mass.

Electron identification is assisted by two additional subdetectors located within the central calorimeter systems: the central electron strip chambers (CES) and the central pre-radiator $(\mathrm{CPR})$.

\section{CES}

The Central Electron Strip chamber is a gas proportional strip and wire chamber inserted between the eighth lead layer and ninth scintillator layer of the CEM, a position corresponding to the average depth at which the transverse development of an electromagnetic shower is at its maximum ${ }^{4}$. The CES is much more finely segmented than the towers of the CEM, allowing close matching of tracks to electromagnetic showers.

Charge from shower ionization is collected on orthogonal wires and strips, providing a local $x$ and $z$ position within each wedge, along with a measurement of the

\footnotetext{
${ }^{4}$ This depth is calculated to include the contribution of the solenoid to the total electromagnetic interaction lengths.
} 


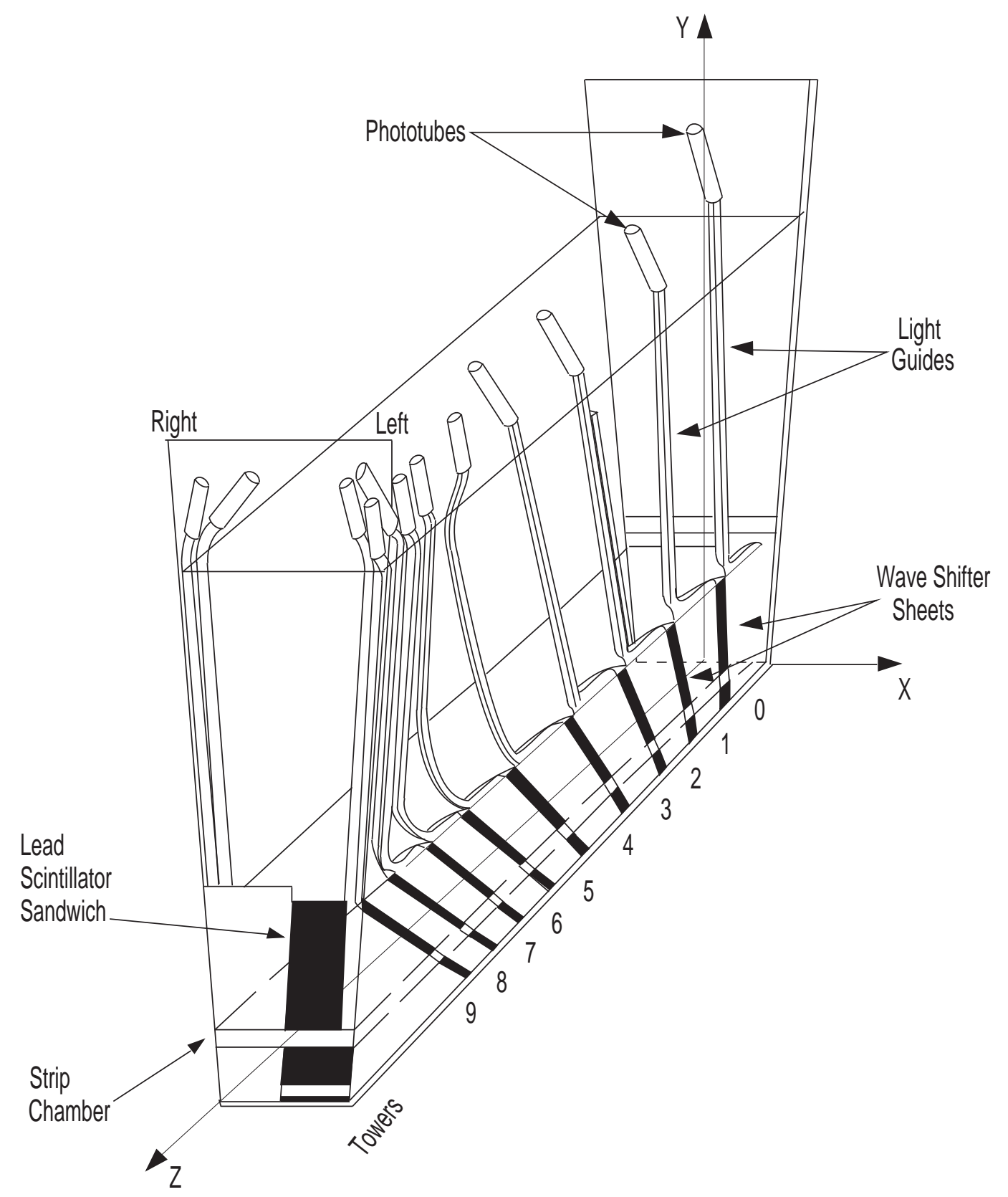

Figure 2.7: Schematic of the light-gathering system in a central calorimeter wedge, showing the position of the CES within the CEM. 
Figure 2.8: A schematic diagram of the CES strips and wires.

shower shape, which can be compared to shapes taken from testbeam data to distinguish between single showers from electrons or photons and closely-spaced double showers from $\pi^{0} \rightarrow \gamma \gamma$.

The CES is an important part of electron identification; it is used for all electrons in this analysis. Hadron rejection based on CES information allows operation of the Level 2 single electron trigger at an increased bandwidth. The track-to-cluster matching and shower shape $\chi^{2}$ are part of both the trigger electron and soft electron quality cuts (see Sections 3.4.1 and 7.2.1). The fine segmentation of the CES allows tight quality cuts with little dependence on the separation of the electron candidate from other tracks or calorimeter energy.

\section{CPR}

While the CES measures electromagnetic showers at their maximum transverse extent, the Central Pre-Radiator samples the early development of electromagnetic showers. The CPR consists of proportional chambers located between the solenoid and the CEM (see Figure 2.2). The chambers are parallel to the beam axis and 
provide $r$ - $\phi$ position measurements only. The energy deposited on the wires is measured in units of the charge collected $\left(Q_{C P R}\right)$. The CPR is not used in the Level 2 electron trigger or in the definition of trigger electrons; nevertheless, it provides an excellent electron-hadron separation which is very useful for the identification of soft electrons (see Section 7.2.1).

\subsubsection{Muon Detectors}

Muons, like electrons, play a critical role in this analysis. Muons are unique in experimental particle physics because of their relatively long lifetime ${ }^{5}$ coupled with their lack of strong interactions with the material of the detector. Thus, unlike the charged hadrons, muons are minimum-ionizing particles, capable of penetrating large quantities of matter. The principle of muon detection is to place detectors behind some volume of dense absorber and define any charged particle which succeeds in penetrating it to be a muon. There is a balance to be struck in selecting the amount of absorber, as muons can "range out" in material as they lose energy by scattering. Thicker and/or denser absorbers give better hadron rejection but reduce sensitivity to low-momentum muons.

The CDF muon system consists of four detector subsystems which employ this principle. The first and third to be described use the material of the calorimeters as their absorbers; the second has additional steel shielding. The fourth system, the forward muon chambers (FMU), are not used by this analysis and will not be described here, though they are placed at the forward/backward toroids as shown in Figure 2.3. The muon systems are integrated into the trigger system, and are designed to provide both position and momentum information to the trigger to aid

\footnotetext{
${ }^{5}$ The muon lifetime as measured in its rest frame is $2.197 \times 10^{-6} \mathrm{~s}$, giving a $\mathrm{c} \tau$ of $658.7 \mathrm{~m}$; the apparent lifetime of energetic muons is enhanced by relativistic effects.
} 
its decision. The $\eta-\phi$ coverage of the three "central" muon chambers is shown in Figure 2.9.

\section{CMU}

The central muon chambers (CMU) [49] consists of wire chambers just outside the central calorimeters; the steel in the calorimeters provides five hadronic absorption lengths of material. The range-out of muons for this thickness is approximately $1.4 \mathrm{GeV} / \mathrm{c}$. There are three muon towers in each calorimeter wedge, covering $12.6^{\circ}$ of the $15^{\circ}$ wedge. The total $\phi$ coverage of the $\mathrm{CMU}$ is therefore $85 \%$; the $\mathrm{CMU}$ is instrumented over $|\eta|<0.6$. Each muon tower is composed of four layers as shown in Figure 2.10. The sense wires in each cell are staggered, allowing the readout of layers $1 \& 3$ and $2 \& 4$ to be ganged together without confusing hit associations. This is done to reduce the amount of readout electronics connected to the chamber.

A series of hits in the layers is assembled into a track segment as is done in the tracking chambers. Muon track segments are called stubs. The position and slope of a stub are taken from the timing information of the hits. The position yields an $r-\phi$ measurement; the slope is defined to be the larger of $t_{4}-t_{2}$ and $t_{3}-t_{1}$, and corresponds to the $p_{\mathrm{t}}$ of the muon candidate. The wires are read out on both ends of the cell, the division of charge between each end provides an $r$-z

position measurement. Thus the stub is a fully three-dimensional measurement of the muon's position and direction, which can be matched to tracks found in the CTC. The agreement between the track and the stub is a measure of a muon's quality; cuts on the "matching variables" are used to ensure good muon selection. Though the track-stub matching has just been described as performed for CMU stubs, the same procedure is applied to all muon chambers. 


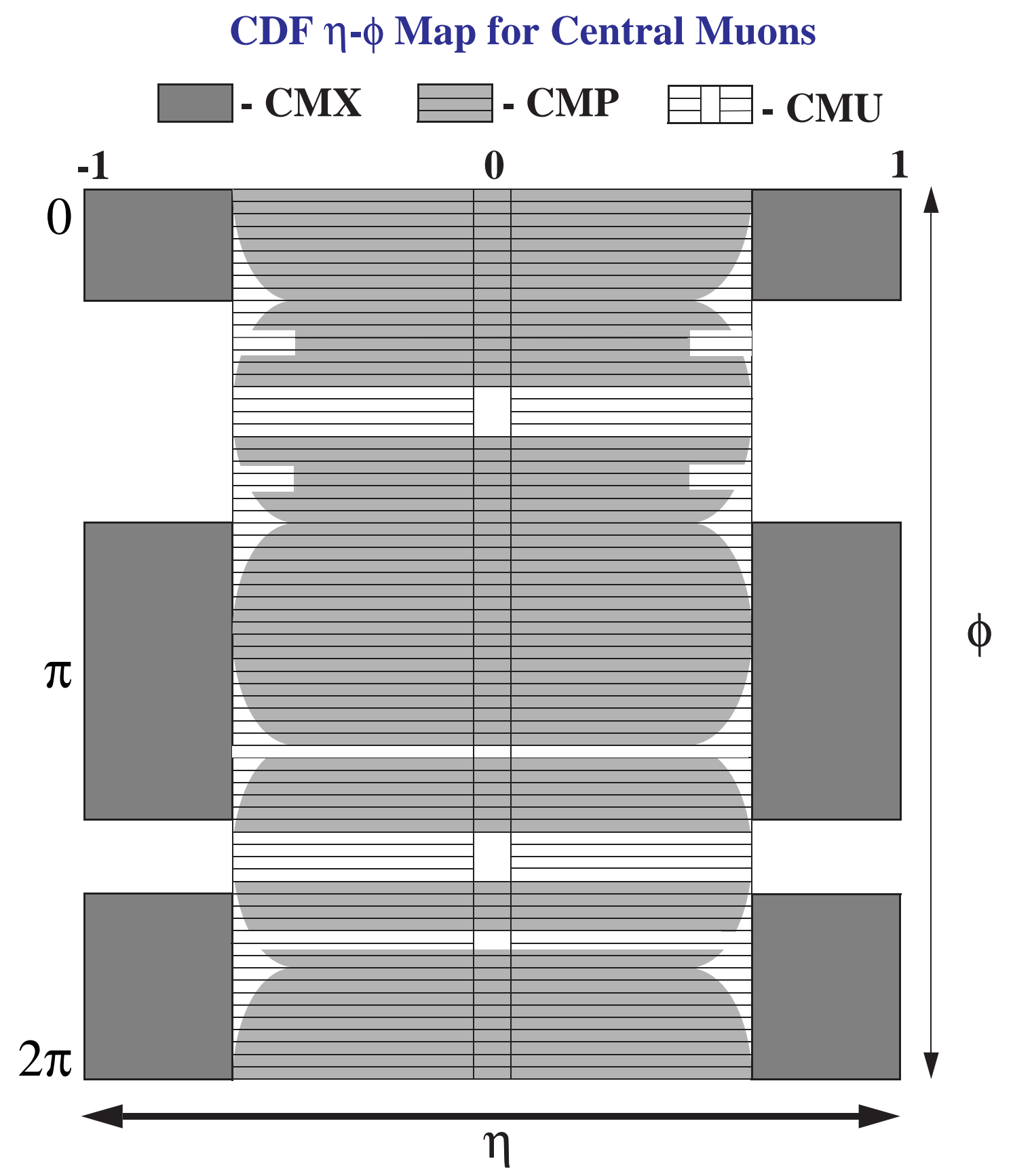

Figure 2.9: The $\eta$ - $\phi$ coverage of the three muon detector systems. Note the substantial overlap between the CMU and CMP. 


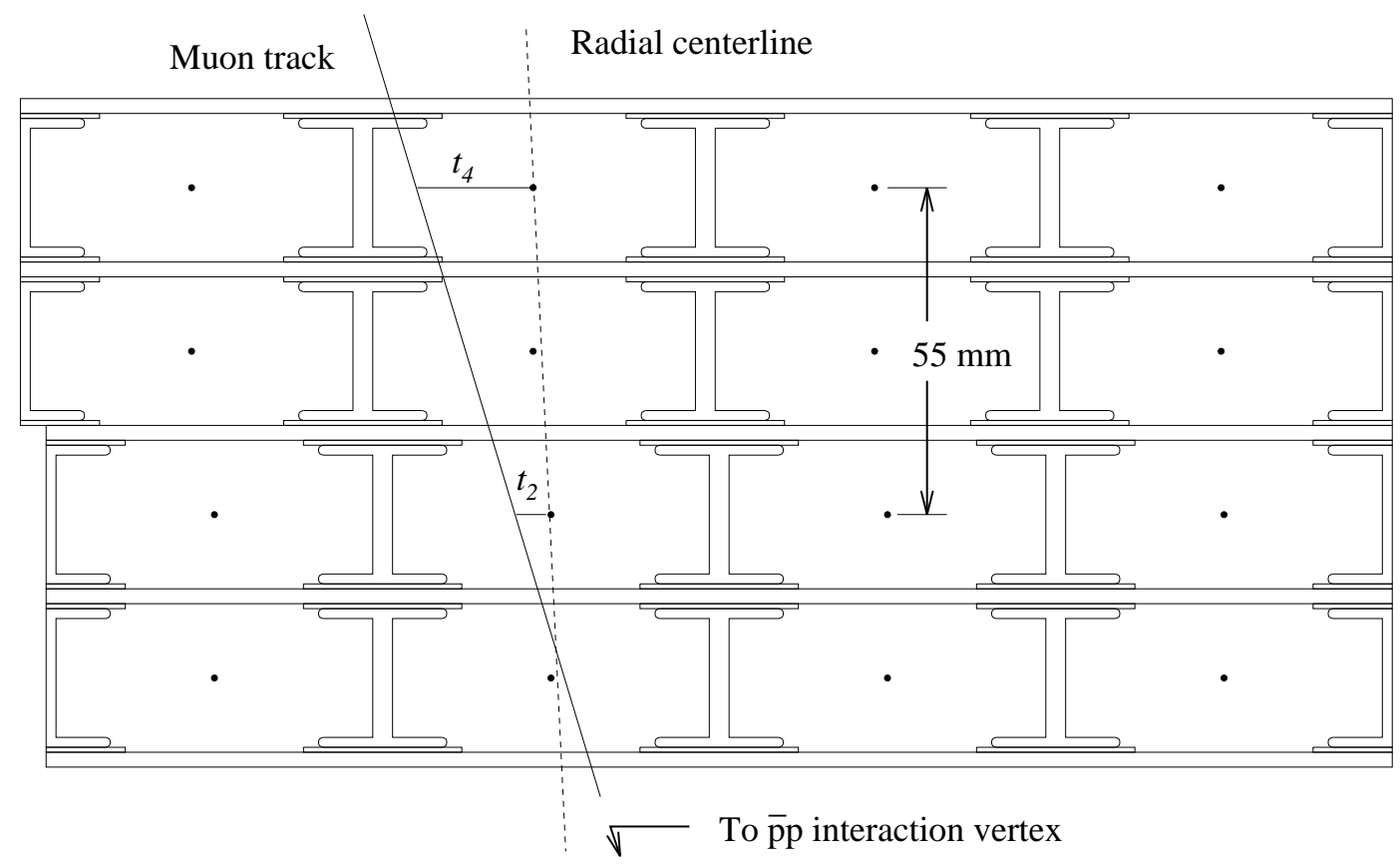

Figure 2.10: Four layers of a central muon chamber tower.

\section{CMP}

Since the calorimeters provide only five hadronic absorption lengths, some hadrons may not shower in the calorimeter, and can "punchthrough", faking the signature of a muon by leaving hits in the CMU. The central muon system was upgraded at the commencement of Run $1 \mathrm{~A}$ to provide additional muon chambers (CMP) with significant geometric overlap with the CMU, behind three more hadronic absorption lengths of steel. The muon range-out to the $\mathrm{CMP}$ is $p_{\mathrm{t}}=2.6-2.8 \mathrm{GeV} / \mathrm{c}$. The $\mathrm{CMP}$ is instrumented over $|\eta|<0.6$, like the CMU, and covers $80 \%$ of $\phi$; a portion of the $\phi$ gaps in the CMU are covered by the CMP. This analysis will make use of the small numbers of muons whose tracks are only matched to CMP stubs, but the advantage of the large overlap is that the best quality muons are those whose tracks are matched to both CMU and CMP stubs. 


\section{CMX}

Another Run 1A muon upgrade was the installation of the central muon extension chambers (CMX), which increased the $|\eta|$ coverage from 0.6 to 1.0. The $\phi$ coverage is $80 \%$, and approximately six hadronic absorption lengths of shielding is provided by the plug calorimeters. Despite the larger amount of shielding compared to the CMU, operation of the CMX during Run $1 \mathrm{~A}$ was hampered by particles scattering from the beampipe assembly, bypassing the shielding and creating numerous fake hits in the CMX. The beampipe was replaced by another with less material before the commencement of Run 1B, so the CMX muon candidates presented in this analysis are of the higher quality planned for in the CMX design. The geometric overlap between the CMX and the other muon chambers is negligible; muon candidates matched to stubs in the CMX and any other chamber are rejected as being poorlymeasured tracks whose quality cannot be guaranteed.

\subsubsection{Trigger System}

With a bunch-crossing time of $3.5 \mu \mathrm{s}$ and 1-2 $p \bar{p}$ interactions per crossing, the Tevatron provides at least 300,000 collisions per second. The vast majority of these interactions are "uninteresting" QCD-only light quark and gluon jet production. As data can be recorded at a rate of only $\sim 5 \mathrm{~Hz}$, a trigger system is used to select the "interesting" events from the total in an efficient manner 6 . The trigger is divided into three stages, or levels, which progressively accept fewer and fewer events to meet the total data acquisition (DAQ) bandwidth. Later levels can make use of the additional time afforded by the reduced rates from earlier levels to calculate quantities of interest from the raw detector information.

\footnotetext{
${ }^{6}$ While QCD processes are (in fact) interesting, and an important part of CDF's physics program $[50,51]$, the lack of a trigger system would limit CDF to only a QCD program.
} 
As the cross-sections for various physical processes can be very different, the DAQ bandwidth is "budgeted" among them by imposing prescaling — that is, some triggers are allowed to pass only a certain fraction of those events which meet their requirements. Triggers designed to accept events from processes with small cross-sections (e.g. $t \bar{t}$ or $W$ production) are assigned low prescale factors (or none at all); those with large cross-sections (e.g. low-energy dijet production) are assigned high prescale factors. Dynamic prescaling of Level 2 triggers was adopted during Run 1B, in which prescales were adjusted depending on the total Level 2 output rate. During periods of high instantaneous luminosity, prescales were increased to prevent the total rate from growing too large.

\section{Data Acquisition}

The various detectors are read out by front-end electronics, which pass signals to digitization modules for assembly into an event record. The front-end includes almost as many subsystems as there are detectors. FASTBUS time-to-digital converters (TDCs) handle tracking and prompt muon data. RABBIT cards read out calorimeter and the remaining muon chamber information. The SVX sequencers control the operation of SVX'; they are paired with the digitizers which take the analog per-channel charge and address as input. Data from the front-end is sorted and assembled into a single record by the event builder. A record is approximately $200 \mathrm{~KB}$ in size. Should the event pass all three levels of the trigger system, the record (along with information about the trigger decisions) is written to tape.

The larger luminosity conditions of Run $1 \mathrm{~B}$ and Run $1 \mathrm{C}$ compared to Run 1A required some modifications to the triggers to ensure a maximal DAQ livetime. The recording bandwidth did not vary significantly during Run 1 . Consequently, the trigger cross-sections had to be reduced during Run 1B. Quality requirements were 
tightened and additional information was made available to the trigger hardware to aid in its decision. While more sophisticated trigger hardware was added in the middle of Run 1B, it did not greatly increase the capacity, but rather enabled additional specialty triggers to be added to the system.

\section{Level 1}

The Level 1 trigger lowers the event rate to $\sim 1 \mathrm{kHz}$ by selecting events with large calorimeter energy deposits or hits in the muon chambers. Lepton triggers are divided into single lepton and dilepton triggers; the former require higher lepton $p_{\mathrm{t}}$ or $\mathrm{E}_{\mathrm{t}}$ thresholds than the latter, owing to both the larger cross-section and larger fake rate. Single central electron and photon candidates are identified by a electromagnetic calorimeter tower with an energy above $8 \mathrm{GeV}$. Single muon candidates are identified by hits in the muon chambers with a $p_{\mathrm{t}}$ (as measured by the slope of the stub) above $6 \mathrm{GeV} / \mathrm{c}$. The dilepton trigger thresholds are $4 \mathrm{GeV}$ for electrons and $3.3 \mathrm{GeV} / \mathrm{c}$ for muons.

The basic requirements of the Level 1 triggers accepting most of the events used by this analysis are:

- L1_CALORIMETER (no prescale): accepts events with a single calorimeter tower above a specified threshold; the threshold for CEM towers is $8 \mathrm{GeV}$.

- CMU_CMP_6PT0_HTDC_BBC (no prescale): requires a $p_{\mathrm{t}}>6 \mathrm{GeV}$ central muon stub with strict requirements to assure the chamber hits are in time with a bunch crossing.

\section{Level 2}

The Level 2 trigger accepts those events passing one or more Level 1 triggers. Specific Level 2 triggers may require specific Level 1 triggers. More information is available to Level 2. Neighboring towers with deposited energy are clustered together. The central fast tracker (CFT) assembles CTC hits into track candidates with rough 
$p_{\mathrm{t}}$ estimates. The CFT has a momentum resolution of $\delta p_{\mathrm{t}} / p_{\mathrm{t}} \approx 0.035 \times p_{\mathrm{t}}(\mathrm{GeV} / \mathrm{c})$ and has an efficiency of $93.5 \pm 0.3 \%$ for isolated tracks with $p_{\mathrm{t}}>10 \mathrm{GeV} / \mathrm{c}$. Neural networks are employed to identify photon and $\tau$ candidates, and CES information can be used to select electron or photon candidates.

The energy deposition of electron and photon candidates is required to be mostly in the electromagnetic calorimeter. In addition, the CES can be checked for an energy cluster consistent with an early-showering particle. The Level 2 single lepton triggers require a minimum match in $\phi$ between a CFT track and a calorimeter cluster or a muon stub. Dilepton triggers require either one or both leptons to be so matched. Other Level 2 triggers include event selection based upon isolated energy, missing $\mathrm{E}_{\mathrm{t}}$, or tracks plus energy (designed for $K^{\star} \gamma$ candidates).

The basic requirements of the Level 2 triggers accepting most of the events used by this analysis are listed below. The Level 2 dynamic prescaling was made dependent on the total Level 2 output rate by defining three bins of the rate (0$12 \mathrm{~Hz}, 12-22 \mathrm{~Hz}, 22+\mathrm{Hz}$ ) and a prescale factor for each (e.g. 1:2:8).

- CEM_8_CFT_7_5_XCES (prescaled by 1:2:8): requires a CEM energy cluster of $8 \mathrm{GeV}$, a matching CES cluster, and a $7.5 \mathrm{GeV} / \mathrm{c}$ CFT track pointing at both.

- CMUP_CFT_7_5_5DEG (prescaled by 1:2:8): requires a $7.5 \mathrm{GeV} / \mathrm{c}$ CFT track within $5^{\circ}$ in $\phi$ of both a CMU and a CMP stub; a $6 \mathrm{GeV} / \mathrm{c}$ CMUP Level 1 trigger is a prerequisite.

Other triggers contributing to the data samples or used in studies are:

- CEM_8_CFT_7_5 (prescaled by 4:16:64): requires a CEM energy cluster of $8 \mathrm{GeV}$ and a $7.5 \mathrm{GeV} / \mathrm{c}$ CFT track pointing to the cluster.

- CEM_12_CFT_12_XCES (prescaled by 1:1:2): requires a CEM energy cluster of $12 \mathrm{GeV}$, a matching CES cluster, and a $12 \mathrm{GeV} / \mathrm{c}$ CFT track pointing at both.

- CEM_5_CFT_4_7 (prescaled by 10:40:200): requires a CEM energy cluster of $5 \mathrm{GeV}$ and a $4.7 \mathrm{GeV} / \mathrm{c}$ CFT track pointing to the cluster. 
- CMUP_CFT_12_5DEG (no prescale): requires a $12 \mathrm{GeV} / \mathrm{c}$ CFT track within $5^{\circ}$ in $\phi$ of both a CMU and a CMP stub; a $6 \mathrm{GeV} / \mathrm{c}$ CMUP Level 1 trigger is a prerequisite.

- CMX_CFT_7_5_5DEG (prescaled by 8): requires a $7.5 \mathrm{GeV} / \mathrm{c}$ CFT track within $5^{\circ}$ in $\phi$ of a CMX stub; a $10 \mathrm{GeV} / \mathrm{c}$ CMX Level 1 trigger is a prerequisite. Active for only the first $6.5 \%$ of Run $1 \mathrm{~B}$.

- Various dimuon triggers (prescaled by 1-4): require two muon stubs (of various types), each matched to at least a $2.2 \mathrm{GeV} / \mathrm{c}$ CFT track. An appropriate dimuon Level 1 trigger is a prerequisite.

\section{Level 3}

Level 3 reduces the event rate to meet the $5 \mathrm{~Hz}$ recording bandwidth. Level 3 is implemented in software and performs a full event reconstruction, creating higherlevel physics objects such as tracks, jets (including electrons and photons), and muon stubs from raw detector information. Tracks, for example, are found by pattern-recognition software looking for a helical pattern of wire hits in the CTC, and muon stubs are found in a similar fashion by finding a cluster of hits in the muon wire chambers. Jets are defined by clustering calorimeter energy deposited in nearby towers. While this may seem a duplication of the activities of the Level 2 trigger, the Level 3 algorithms are much more sophisticated, allowing better pattern recognition and matching of track segments and energy clusters between different detectors. In addition, $p_{\mathrm{t}}$ and $\mathrm{E}_{\mathrm{t}}$ thresholds are much better defined.

Second-order objects are also created. A muon candidate is created by projecting a track through the detector to the muon chambers, and matching it to an existing muon stub. An electron candidate consists of a calorimeter cluster, a CES cluster, and a CTC track, matched together. A photon candidate is essentially an electron candidate with no matched track. The shower shape in the CES is also examined to ensure consistency with a single particle, rather than the wider shape from $\pi^{0} \rightarrow \gamma \gamma$. 
The Level 3 trigger operates after reconstruction by selecting events based upon these second-order quantities. The basic requirements of the triggers accepting most of the events used by this analysis are:

- ELEB_CEM_8_6 (no prescale): requires an electron with clustered CEM $\mathrm{E}_{\mathrm{t}}>7.5 \mathrm{GeV}$ and a track of $p_{\mathrm{t}}>6 \mathrm{GeV} / \mathrm{c}$ pointing at the cluster.

- MUOB_CMU_CMP_8 (no prescale): requires a track of $p_{\mathrm{t}}>8 \mathrm{GeV} / \mathrm{c}$ matched to both CMU and CMP stubs.

Other triggers contributing to the data samples or used in studies are:

- ELEB_CEM_8 (prescaled by 25): requires an electron with clustered CEM $\mathrm{E}_{\mathrm{t}}>7.5 \mathrm{GeV}$, and does not specifically require a track, though the Level 2 trigger CEM_8_CFT_7_5_XCES (which does) is a prerequisite.

- ELEB_CEM_5_5 (no prescale): requires an electron with clustered CEM $\mathrm{E}_{\mathrm{t}}>5 \mathrm{GeV}$ and a track of $p_{\mathrm{t}}>5 \mathrm{GeV} / \mathrm{c}$ pointing at the cluster, and requires CEM_5_CFT_4_7 at Level 2.

- MUOB_CMX_8 (no prescale): requires a track of $p_{\mathrm{t}}>8 \mathrm{GeV} / \mathrm{c}$ matched to a CMX stub. It was active for the only first $6.5 \%$ of Run $1 \mathrm{~B}$.

- PSIB_DIMUON_JPSI (no prescale): requires two muons (any type), oppositely charged, with invariant mass $2.7<M_{\mu \mu}<4.1$ (thus including $\left.\psi^{\prime} \rightarrow \mu \mu\right)$.

\section{Stream B}

The data passing Level 3 is distributed among three paths, or streams. The streams were established to give priority in data accessibility to more "vital" triggers. Stream A includes triggers used for detector and trigger calibrations as well as the most urgent analyses (such as top and $W$ studies). Stream A triggers, comprising high-energy jets, photons, and single leptons, and the highest-quality $J / \psi \rightarrow \mu \mu$ events, also have some of the lowest prescales, further indicating their importance to the experiement. Stream B consists of the lower-energy jet, photon, and lepton events, while Stream C consists of lepton triggers with the loosest requirements as well as minimum-bias interactions. 
The data from the low- $p_{\mathrm{t}}$ single lepton triggers used by this analysis were directed to Stream B. It must be emphasized that the streams only define the order in which data is processed (as described in Section 3.2). Every event recorded by Level 3 is ultimately made available to experimenters. 


\section{Chapter 3}

\section{Primary Datasets}

A dataset is a set of recorded events used in an analysis. Colloquially, it may refer to the entire set of events passing a particular trigger or a set of triggers, a subset of events which meet some initial quality requirements (here called the "primary" dataset), or a further subset meeting the full event selection, comprising the final sample on which the analysis is performed. Which one is meant will be either obvious from the context or directly specified. "Sample" is often used interchangeably with "dataset". This chapter describes how the primary datasets for this analysis were selected from data passing particular Level 3 triggers.

\subsection{The 1994-1995 Run}

This analysis uses data from the second portion of the Tevatron collider Run 1 (known as Run 1B). CDF has defined the portion of valid data to be that taken between January 22, 1994 and July 24, 1995; this constitutes $86.3 \mathrm{pb}^{-1}$ of data recorded onto $8 \mathrm{~mm}$ tapes. 


\subsection{Event Production}

Those events that have been recorded by the data acquisition system are reprocessed offline by the "production farms". A farm is a collection of computers which reads events from tape, redoes the full event reconstruction of the Level 3 trigger, and returns the output back to tape. The disk and tape resources are handled by a "server" node, and the processing handled by a "worker" node. For Run 1B production, CDF used two farms: a cluster of IBM computers consisting of 3 server nodes and 34 worker nodes, and a cluster of Silicon Graphics computers consisting of 1 server node and 63 worker nodes. Together, the two farms can process approximately 1.3 million events per week at full capacity.

This offline event reconstruction is called "production". The software used in production is essentially the same as that used by the Level 3 trigger, with one important difference: production has access to the offline calibration databases, which contain appropriate detector constants derived from the data after it is recorded. Thus the quality of the higher-level objects from production is slightly better than those directly from Level 3. The Run 1B data was processed using version 7.11 of the offline production. The final data samples for this analysis were reprocessed with a portion of version 7.12 production; this will be described in more detail in Section 4.1.

It bears repeating that data from all three streams are processed by production. The assignment of a Level 3 trigger's output to a particular stream only indicates the order in which it is processed.

The full output of production consists of a very large event record containing the raw detector information, the trigger decisions, and the reconstructed quantites created both by Level 3 and by production. The data are also made available without the raw detector information and Level 3 reconstruction. Most analyses 
(including this one) depend only on production-derived quantities.

\section{3 $\quad$ BPAD processing}

Events from the output of Stream B production were split into many sub-streams based upon which Level 3 triggers were passed, in order to facilitate analyses: physicists working on a $B_{s}^{0}$ mass measurement would not be interested in reading the large quantities of QCD multi-jet data recorded by CDF. Of course, if an individual event passed many different triggers, copies of this event would be sent to each of the representative "split streams". Nine of these streams were identified as relevant to $b$ physics analyses: the electron-muon, the high- and low- $p_{\mathrm{t}}$ inclusive electrons, the high- and low- $p_{\mathrm{t}}$ inclusive muons, and the various dimuon split streams (high-mass dimuons, low-mass dimuons, the $\Upsilon \rightarrow \mu \mu$, and the $J / \psi, \psi^{\prime} \rightarrow \mu \mu$ ). This analysis relies on the data in the low- $p_{\mathrm{t}}$ inclusive electron and muon split streams; the chief triggers accepting data for these streams are described in Section 2.2.4, and further reviewed in this chapter.

As a practical matter, reading large datasets can be difficult. Users can be interrupted by the occasional tape drive failure, made more likely by the large number of individual tapes that must be read, or can find that a specific tape they require is already in use by another user, which is likely as the number of users interested in these nine datasets is known to be large. Many analyses are very CPU-intensive, which only increases the annoyance of these interruptions. In addition, most analyses share many common features, and requiring each user to run the same code is a net waste of computing resources.

Accordingly, it was determined to subject each of the nine split streams to further processing to reduce the event record size and make the data available on a more 
convenient medium than $8 \mathrm{~mm}$ tapes. The additional processing included storing the run-by-run $p \bar{p}$ beamline information in the event record (saving the user a series of database queries); collecting the information on which triggers each event passed from the various storage locations in the event record and summarizing it in one place; requiring the event to pass the same requirements as the Level 3 trigger, based on quantities calculated by production; tightening the muon quality requirements

to ignore muons of dubious worth; and a number of steps to reduce the size of the event record, all based on deleting information not of interest. This new event record format is known as BPAD.

The BPADs were sent to robotic tape storage media. The STK silo, operated by Fermilab's Computing Division, has a maximum capacity of 4 Terabytes and 157 Gigabytes of staging disk. The directory of stored files is maintained by the CERN software product FATMEN. Requested files are staged quickly to the NFS-mounted disks, eliminating the need for users to submit a series of failure-prone $8 \mathrm{~mm}$ tape mount requests; files which have not been accessed in some time are deleted to create space for newly-requested files.

\subsubsection{Inclusive electrons (ECLB)}

The stream B inclusive electron trigger data were sent to the ECLB and ECMB split streams by production, and further processed into BPADs. Loosely speaking, the former contains the output of the $8 \mathrm{GeV}$ electron triggers, and the latter the output of the $18 \mathrm{GeV}$ single electron triggers. Trigger electron data for this analysis were taken from ECLB.

Over the course of Run 1B, events passing one or more of four Level 3 triggers (ELEB_CEM_8_6, ELEB_CEM_8, ELEB_CEM_5_5, and ELEB_KSTAR_GAMMA) were split to ECLB. The last trigger, a specialty trigger for $B^{0} \rightarrow K^{0^{*}} \gamma$ studies, 
was introduced two-thirds of the way through Run $1 \mathrm{~B}$ and directed to the ECLB stream by production for convenience. Data from this trigger was split to a separate output stream (KSGB) at the BPAD-processing stage - the ECLB BPADs only contain data from the first three Level 3 triggers. Of these, the data are dominated by ELEB_CEM_8_6. Both ELEB_CEM_8 and ELEB_CEM_5_5 have large prescales, whether applied by Level 3 (in the case of the former) or by a Level 2 prerequisite trigger (in the case of the latter). Of the events which do pass ELEB_CEM_8, the vast majority also pass ELEB_CEM_8_6; the extra contribution is negligible.

The production output of ECLB was 6,078,693 events; after BPAD processing, 5,627,210 remained. The difference is largely due to KSGB events which do not pass any of the three electron triggers, and are removed. For electron events, the BPAD inefficiency is $1 \%$, owing to the slight differences between Level 3 and production. Hereafter, "ECLB" should be taken to refer to the BPAD split sample.

\subsubsection{Inclusive muons (MULB)}

As in the case of the inclusive electron data, the stream B inclusive muon trigger data were sent to the MULB and MUCB split streams by production, and further processed into BPADs. Loosely speaking, the former contains the output of the $8 \mathrm{GeV}$ single muon triggers, and the latter the output of the $15 \mathrm{GeV}$ single muon triggers. Trigger muon data for this analysis were taken from MULB.

Over the course of Run 1B, events passing either MUOB_CMU_CMP_8 or MUOB_CMX_8 were split to MULB. The latter trigger was only active for the first $6.5 \%$ of the data collected during Run $1 \mathrm{~B}$. The production output of MULB was 2,630,227 events; after BPAD processing, 2,061,789 remained. The BPAD inefficiency of $21.6 \%$ is due to the inclusion of tighter muon quality cuts. As mentioned before, the cuts were tightened to reduce the overall size of the sample; they are 
not tighter than any used in this analysis, and therefore do not incur any loss of statistics. Hereafter, "MULB" should be taken to refer to the BPAD split sample.

\subsection{Trigger Lepton Selection}

From the BPAD datasets, the next step in the creation of the primary samples is to determine the subset of BPAD events that have high-quality single electrons or muons. This effort is made to reduce the number of "fakes" — hadrons which leave signatures in the detector not unlike those of real leptons. At this stage, the physical process which generated the leptons is of no consequence, with one notable exception in the case of electrons. Most of the individual identification cuts used are similar or identical to the ones employed by the Level 3 trigger. The additional requirements ensure the quality of the leptons.

\subsubsection{Trigger Electron Selection}

All trigger electrons used in this analysis are required to be central - that is, they must have a cluster of energy in the CEM, with an identified track in the CTC pointing to the cluster. The selection criteria which match those of the Level 3 trigger are: clustered electromagnetic $\mathrm{E}_{\mathrm{t}}>7.5 \mathrm{GeV}$, track $p_{\mathrm{t}}>6.0 \mathrm{GeV} / \mathrm{c}$, and the

3-tower energy sharing ( $L_{\text {share }}$, described below) must be less than 0.2 . The track is not required to be in the fiducial volume of SVX'.

The energy sharing is a measure of the transverse profile of energy deposition in the neighboring (in the $z$ coordinate) calorimeter towers; it uses the beam vertex and the position of the energy cluster in the CES to determine a trajectory through the calorimeter, which is subsequently used to calculate a value for the energy sharing between the seed tower (that with the highest energy) and its neighbors. A 


\begin{tabular}{|ll|}
\hline $\mathrm{E}_{\mathrm{t}}$ & $>7.5 \mathrm{GeV}$ \\
$p_{\mathrm{t}}$ & $>6.0 \mathrm{GeV} / \mathrm{c}$ \\
$\mathrm{HAD} / \mathrm{EM}(3-$ tower $)$ & $<0.04$ \\
$L_{\text {share }}(3-$ tower $)$ & $<0.2$ \\
$\chi_{\text {strip }}^{2}$ & $<10.0$ \\
$\chi_{\text {wire }}^{2}$ & $<10.0$ \\
$|\Delta x|$ & $<1.5 \mathrm{~cm}$ \\
$|\Delta z|$ & $<3.0 \mathrm{~cm}$ \\
\hline
\end{tabular}

Table 3.1: Trigger electron quality requirements.

low value indicates that the total energy in the cluster is well-contained within one calorimeter tower, which is more consistent with the electron hypothesis. Larger values indicate a wider spread in energy deposition, consistent with a jet containing large numbers of hadrons.

The identification requirements which differ from those at Level 3 include a very strict cut on the ratio of energy measured in the CHA to that measured in the CEM (HAD/EM), tighter cuts on the shower shape in the strip and wire planes of the CES $\left(\chi_{\text {strip }}^{2}\right.$ and $\left.\chi_{\text {wire }}^{2}\right)$, and tighter matching between the energy-weighted wire and strip energy clusters in the CES (providing an $x$ and $z$ position) and the projected track location $(|\Delta x|$ and $|\Delta z|)$. These and the previous cuts are summarized in Table 3.1. From the BPAD sample, 3,374,179 events have one or more electrons which satisfy the requirements.

The electrons selected by these cuts will have passed the Level 3 trigger. While this is not true a priori, the re-imposition of Level 3 requirements on production quantities by the BPAD processing guarantees that this is the case. No effort is made, however, to ensure that any particular electron selected by these requirements was responsible for either a Level 1 or a Level 2 electron trigger, though an examination finds that $95 \%$ were.

The described cuts do an excellent job of ensuring high-quality electrons. There 


\begin{tabular}{|l|ll|l|}
\hline \multicolumn{1}{|c|}{ Quantity } & Cut Value & \multicolumn{1}{c|}{ Definition } \\
\hline$R_{\text {conv }}$ & $>$ & $-10.0 \mathrm{~cm}$ & radius of conversion \\
$R_{\text {conv }}$ & $<$ & $50.0 \mathrm{~cm}$ & radius of conversion \\
$|\Delta \cot \theta|$ & $<$ & 0.03 & \\
$|\Delta z|$ & $<$ & $2.0 \mathrm{~cm}$ & \\
$|\Delta \phi|$ & $<$ & 0.01 & \\
$|\Delta S|$ & $>$ & $0.2 \mathrm{~cm}$ & distance of closest approach \\
Pointing residual & $<$ & $0.2 \mathrm{~cm}$ & consistency of the pair \\
& & & having an origin at the primary \\
\hline
\end{tabular}

Table 3.2: Conversion partner selection requirements. The differences between the track parameters are calculated at the radius of conversion.

is one source of real electrons, however, that should be excluded at this stage. Highenergy photons can undergo conversion to electron-positron pairs in the material of the detector; estimates [52] are that approximately $\sim 30 \%$ of the electrons passing the Level 3 trigger come from conversions. As this analysis is concerned with electrons from $b$ decay, an additional kinematic cut is applied to reject electrons consistent with a conversion origin.

The cut is in the form of a search for a conversion partner to the trigger electron candidate. The signature for a conversion pair is two tracks, of opposite charge, which meet at some point in space, and at that point are parallel to each other, before the magnetic field in the CTC causes them to separate. Detector resolution effects dominate the measurement of the opening angle between the two tracks. The cuts are defined in Table 3.2. The six quantities are calculated for every track paired with the electron track; should any pair pass all of the requirements, the electron is flagged as coming from a conversion, and is rejected. Of the 3,374,179 events with one or more good electrons, 747,679 (22.2\%) are eliminated by the conversion rejection. 


\subsubsection{Trigger Muon Selection}

Muons can be classified according to the muon detectors in which the candidate track has been matched to stubs. The three detectors (CMU, CMP, and CMX) allow for seven combinations (the eighth being no stub in any detector). Some combinations are common: the geometrical acceptance of the CMP shares a large overlap with that of the CMU, while the CMX has minimal overlap with the other two detectors. The Level 3 trigger only accepts candidates with both CMU and CMP stubs, or only a CMX stub; the definition of "trigger muon" is necessarily the same.

The trigger muon quality cuts are the same as in Level 3, with two exceptions: the energy deposited in the CHA calorimeter tower traversed by the candidate track must be greater than $0.5 \mathrm{GeV}$, consistent with a minimum ionizing particle, and the $p_{\mathrm{t}}$ threshold is lower, accepting any muons above $6 \mathrm{GeV} / \mathrm{c}$. The lower $p_{\mathrm{t}}$ cut has a negligible effect; the inclusion of muons between 6 and $8 \mathrm{GeV} / \mathrm{c}$ adds only $0.2 \%$ more events to the sample. The cuts are summarized in Table 3.3.

As in the case of the trigger electron selection, no specific trigger requirements from any level are made. A key difference, however, is that while all trigger electrons will have passed Level 3, it is possible that some selected trigger muons will not, as the CMX muon Level 3 trigger was only active for a portion of Run 1B. "Volunteer" CMX muons, not responsible for a trigger, can be accepted by these requirements, though their contribution is minimal. Only $2.5 \%$ of the accepted muons are CMX muons, in good agreement with the expected contribution from the CMX trigger, which was active for the first $6.5 \%$ of data-taking and "competed" with the CMUP trigger for events during that time.

2,009,040 MULB events have one or more muons which meet these requirements; hereafter, any muon selected in this manner will be referred to as a "trigger muon", 


\begin{tabular}{|c|c|c|}
\hline \multicolumn{3}{|c|}{ All Candidates } \\
\hline$p_{\mathrm{t}}$ & & $6.0 \mathrm{GeV}$ \\
\hline$E_{\text {had }}$ & & $0.5 \mathrm{GeV}$ \\
\hline \multicolumn{3}{|c|}{ CMU - CMP Candidates } \\
\hline$\overline{\chi_{x}^{2}(\mathrm{CMU})}$ & & 9.0 \\
\hline$\chi_{z}^{2}(\mathrm{CMU})$ & & 12.0 \\
\hline$\chi_{x}^{2}(\mathrm{CMP})$ & & 9.0 \\
\hline \multicolumn{3}{|c|}{ CMX Candidates } \\
\hline$\chi_{x}^{2}(\mathrm{CMX})$ & & 9.0 \\
\hline$\chi_{z}^{2}(\mathrm{CMX})$ & $<$ & 12.0 \\
\hline
\end{tabular}

Table 3.3: Trigger muon quality requirements.

whether or not it was actually responsible for a DAQ trigger at any level. Almost all of the trigger muons were those selected by Level 3; a study of the sample shows that $85 \%$ of the trigger muons caused a Level 2 single muon trigger.

\subsection{Primary Dataset Summary}

The primary datasets consist of 2,626,500 ECLB events found with at least one nonconversion trigger electron and 2,009,040 MULB events with at least one trigger muon. Nearly all trigger leptons caused a Level 3 single lepton trigger; a large majority were also responsible for a Level 2 single lepton trigger. The contribution of "volunteer" leptons to the datasets is negligible. 


\section{Chapter 4}

\section{Final Data Samples}

Starting from the primary datasets described in the last chapter, efforts are made to enhance the proportion of trigger leptons coming from $b$ decay by exploiting the relatively long $b$ lifetime. SVX', with its excellent track $r-\phi$ resolution, allows CDF to identify $b$ jets by finding tracks intersecting at vertices inconsistent with the primary vertex. This analysis does this by taking advantage of a software package known as SECVTX [53], originally developed for CDF's top analyses [54, 55]. SECVTX begins with a list of SVX tracks in a given jet, then searches for a "cluster" of tracks which intersect at a point inconsistent with the primary interaction vertex. Cuts on the significance of the separation between primary and secondary vertices and the quality of the secondary vertex increase the purity of the $b$-tagging. This analysis requires that a secondary vertex "tag" is found by SECVTX in the same jet as the trigger lepton.

\subsection{SVX Reprocessing}

It is usually the case that the best knowledge of a detector's performance is obtained from the data. In the case of the $\mathrm{SVX}^{\prime}$ detector, the tracks found by production 
version 7.11 pattern recognition relied on a set of internal alignment parameters (the positions of each ladder relative to the others) derived from optical measurements during construction. Improving the understanding of the internal alignment, as well as establishing the external alignment (the positions of the barrels relative to the CTC), was naturally one of the highest priorities of the $\mathrm{SVX}^{\prime}$ group at the commencement of Run 1B.

To guarantee that this analysis benefits from the fruit of these labors, all data from the primary samples are reprocessed through version $7.12 \mathrm{SVX}^{\prime}$ pattern recognition, using the most recent internal alignment parameters. The matching of SVX' hits to CTC tracks therefore includes the best available knowledge of the detectors' position. This reprocessing is necessary, as production continued to use version 7.11, even after 7.12 became available, in order to maintain a consistent dataset.

The result of using the most recent $\mathrm{SVX}^{\prime}$ internal alignment corrections on an ensemble of tracks is illustrated in Figure 4.1.

\subsection{Primary Vertex Finding}

In order to make a cut on the secondary vertex separation, the primary must first be found. A software package called VXPRIM [56] is widely used at CDF for this purpose. It first constructs a seed vertex, an initial estimate of the primary vertex position, then performs a fit with all acceptable SVX tracks, using the seed's position and uncertainty as a constraint in the fit.

\subsubsection{The Seed Vertex}

The primary vertex estimate starts with a candidate $z$ position (in the longitudinal direction), then uses this to calculate the $x$ and $y$ positions from knowledge of the 


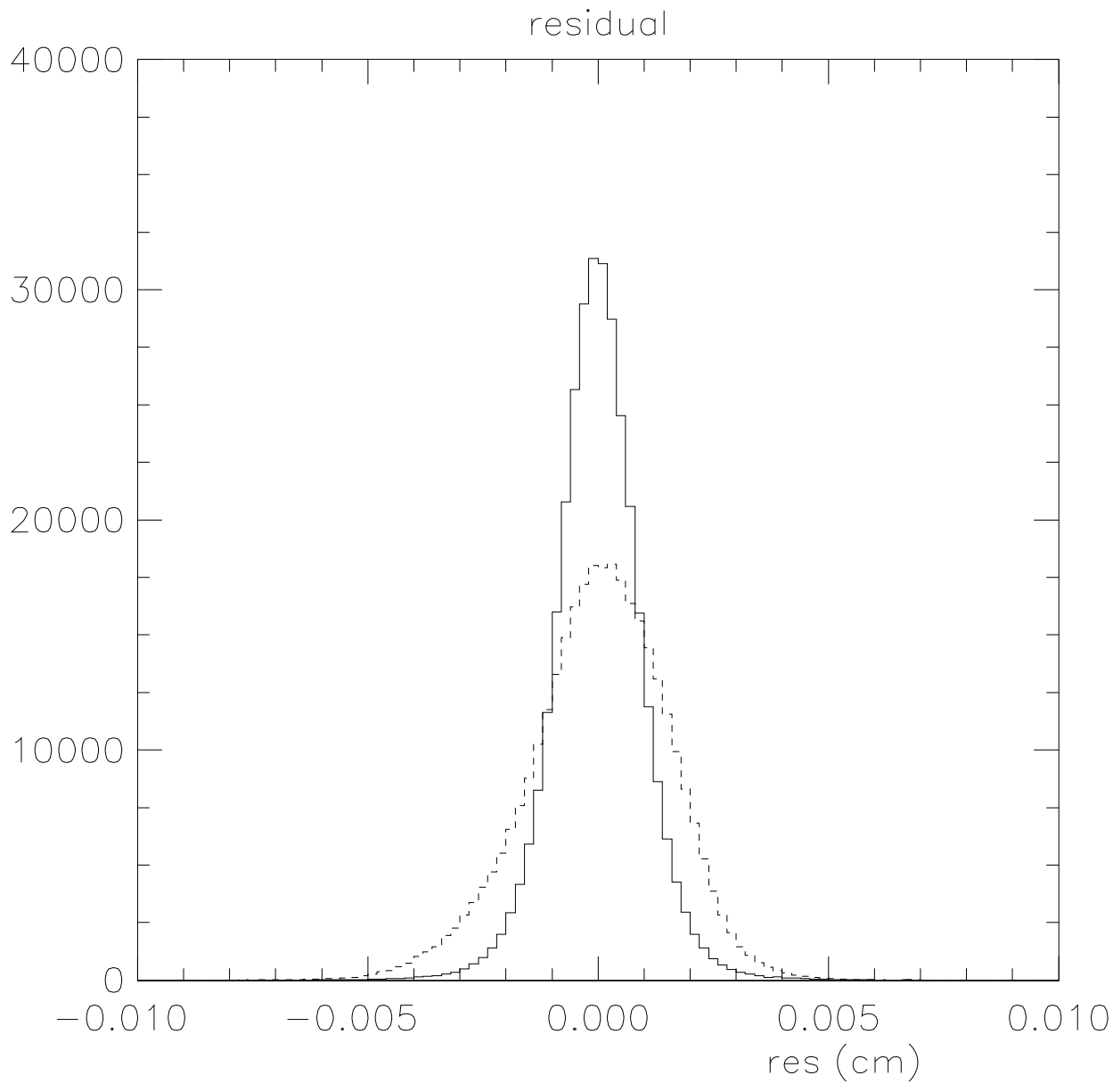

Figure 4.1: Fit residual distributions for $\mathrm{SVX}^{\prime}$ tracks, demonstrating how the use of the 7.12 internal alignment constants (solid) improve the SVX track resolution compared to an earlier set of constants (dashed), based upon the optical alignment (obtained during construction). The use of the best alignment improves the r.m.s. of the residual distribution from $15.3 \mu \mathrm{m}$ to $9.7 \mu \mathrm{m}$. 
$p \bar{p}$ beamline, the transverse location of the $p \bar{p}$ beams versus $z$.

\section{$z$ Position from the VTX}

VXPRIM starts with information stored in the VTVZ bank, which is created in the event record by production. VTVZ records the $z$ positions of interactions based on fits of tracks found in the VTX, the detector with the best $z$-resolution. VXPRIM treats the vertices in VTVZ as candidates for the primary vertex, and relies only on the recorded quality, $z$ position, and uncertainties. It starts with the five highest-quality vertices (though there may be fewer found by production and recorded in VTVZ), and determines which has the largest scalar sum $p_{\mathrm{t}}$ of CTC tracks within $5 \mathrm{~cm}$ in $z$ of their respective positions. The $z$ of the vertex with the largest sum is taken as the $z$ of the seed vertex. The uncertainty on the $z$ position is taken from production's fit of VTX tracks, stored in the VTVZ bank; typical resolutions are on the order of $1 \mathrm{~mm}$.

\section{$x$ and $y$ Positions from the SVX Beamline}

VXPRIM obtains the $x$ and $y$ positions of its seed vertex from the run-averaged SVX beamline position [57]. The beamline database is a record of the $p \bar{p}$ beams' position relative to the detector during each run taken by the DAQ software. The SVX beamline is determined by collecting high-quality SVX tracks in each event, constraining them to come from a common vertex, and taking all such vertices found during a run to fit a straight line to the $x$ and $y$ versus $z$ distributions.

First estimates of the beamline are determined as data is taken, from the first events of a run and immediately after the conclusion of a run; the final beamline database entry is constructed offline, using data processed by production, which has the advantage of the most complete detector calibrations and alignments. The 
beamline database stores not only the beam position, but also the beamspot: the transverse cross-sectional size of the beam, found from the spread in $x$ and $y$ of the found vertices. The beamspot is circular, and approximately $35 \mu \mathrm{m}$ in size. Figure 4.2 shows the beamspot for a particularly well-measured run.

There are some runs where the database entry indicates that the beamline is poorly determined, or there is no entry at all. These runs lack enough events to get a good determination of the beamline; they are typically either very short or from periods where the Tevatron ran with particularly low luminosity. In these cases, VXPRIM will accept the CTC beamline, determined in a similar fashion from only the CTC information of tracks, with the substantially larger uncertainties on the beamline and beamspot which arise from the worse track resolution of the CTC relative to the SVX. The CTC beamline is always available.

VXPRIM uses the selected VTVZ $z$ position to calculate the seed's $x$ and $y$ position from the beamline offsets and slopes. The uncertainty on $x$ and $y$ is taken as the beamspot size, as recorded in the database.

\subsubsection{Primary Vertex Fit}

VXPRIM then generates a list of acceptable SVX tracks which will serve as an input to the fit, along with the seed vertex. The requirements are described in Table 4.1. The cut on each track's impact parameter $(D)$ serves to reject tracks which are inconsistent with coming from a $p \bar{p}$ interaction vertex, i.e. a particle knocked out of the beampipe or from a decay-in-flight. The fit has two components: a first unsteered vertex-constrained fit, followed by a steered vertex-constrained fit, where "steering" indicates whether the track parameters are allowed to be adjusted, within their uncertainties, to minimize the $\chi^{2}$ of the fit.

Both fits are iterated over the list of acceptable tracks. First, all tracks are 
vertexed (subject to the constraint of being consistent with the seed vertex). The track with the largest residual to the fit result is rejected if the residual is larger than 3.5, and the fit repeated as necessary until all tracks have residuals smaller than 3.5. The unsteered fit is performed first to screen out tracks which are actually, but perhaps not obviously, inconsistent with coming from the same point as the other tracks; it is computationally much faster than a steered fit, so less time is wasted eliminating these tracks. If, at any stage of any fit, the number of tracks included in the fit falls to less than 5, the fit will fail, and the output of VXPRIM will be the seed vertex, with its correspondingly larger uncertainty.

\begin{tabular}{|lr|}
\hline \multicolumn{1}{|c|}{ Quantity } & \multicolumn{1}{c|}{ Cut Value } \\
\hline Number of SVX hits & $3 \mathrm{or} 4$ \\
$p_{\mathrm{t}}$ & $>0.4 \mathrm{GeV} / \mathrm{c}$ \\
$|D|$ & $<2.0 \mathrm{~cm}$ \\
$\mid \Delta z($ seed - track $) \mid$ & $<5.0 \mathrm{~cm}$ \\
Fit residual & $<3.5$ \\
\hline Min. \# of acceptable tracks & $\geq 5$ \\
\hline
\end{tabular}

Table 4.1: VXPRIM SVX Track Requirements. $D$ is the impact parameter relative to the CDF origin. 

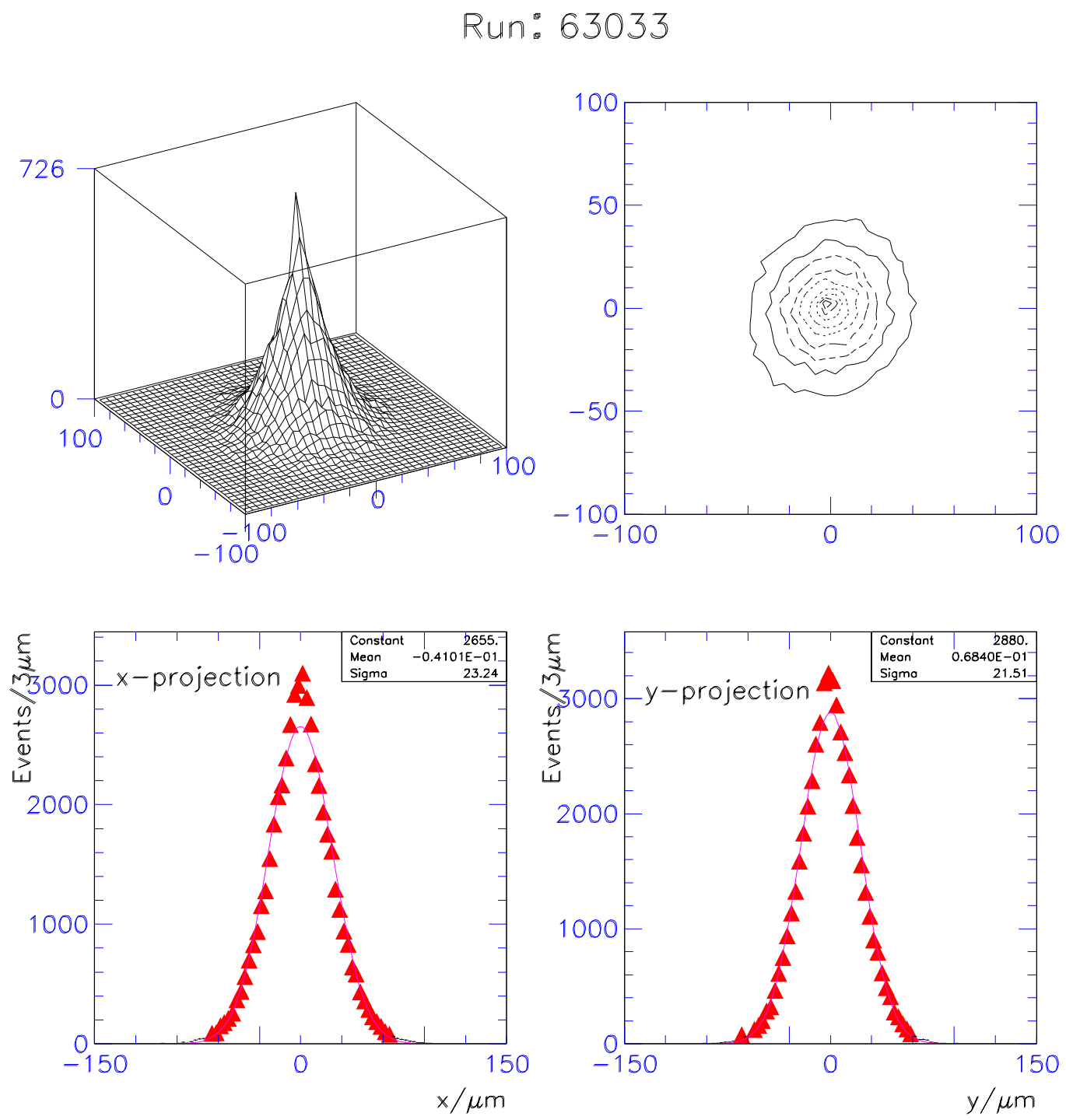

Figure 4.2: Fits to the beamspot for a particular run.

Upper left: The two-dimensional distribution of primary vertices, relative to the beamline.

Upper right: A contour plot of the distribution, highlighting the circular cross-section of the beam.

Lower left: The projection of the distribution along the $x$-axis; the projection has a width of $23 \mu \mathrm{m}$.

Lower right: The projection of the distribution along the $y$-axis; the projection has a width of $22 \mu \mathrm{m}$. 


\subsection{SECVTX $b$-tagging}

SECVTX is a software package developed to identify $b$ jets in candidate top events. It has been demonstrated to have excellent $b$-tagging efficiency and background rejection [54], and is the cornerstone of CDF's top mass and cross-section measurements [55]. The requirement of one or more SECVTX tags in an event, in conjunction with other event selection criteria, sharply reduces backgrounds to $t \bar{t}$ production. The identification of a jet as a $b$ jet also reduces the combinatoric background in fitting the top mass, when constraining two jets in single lepton + jets events to form the $W$ mass. $^{1}$

SECVTX also admirably suits the needs of this analysis, one of the first at CDF to employ SECVTX in a $B_{d}^{0}$ mixing measurement. The requirement that there be a SECVTX tag in the jet associated with the trigger lepton suppresses backgrounds from direct charm production and fake leptons. Some selection criteria are modified to account for the different kinematics of direct $b \bar{b}$ production relative to $t \bar{t} \rightarrow W^{+} b W^{-} \bar{b}$ production, but the high $b$-tagging efficiency and background rejection are maintained. SECVTX is also used in this analysis to estimate the proper time of the $b$ hadron decay from the vertex position of the tag. This is discussed in greater detail in Section 5.2; here, the only concern is describing the SECVTX algorithm and how it is used to select events for the final sample.

\subsubsection{General Algorithm Overview}

The idea of SECVTX is relatively simple, even if the implementation is complex. A list of fiducial jets above a minimum energy is generated, as is a list of the SVX tracks contained in each jet. For the tracks in each jet, SECVTX searches for two or

\footnotetext{
${ }^{1} \mathrm{~A} b$ quark is an unlikely product of the hadronic decay of an on-shell $W$.
} 
more tracks that intersect at a point that is inconsistent with the primary vertex. In the interest of speed and background rejection, not all potential displaced vertices are evaluated. First, SVX tracks are selected by requiring a substantial impact parameter significance, indicating that these tracks are unlikely to have originated at the primary vertex. Second, the tracks are ordered by their quality (see Figure 4.4 for details), and "seed" vertices are constructed using the best tracks first; should an acceptable seed vertex be found, tagging attempts will cease even if the final vertex is unacceptable, under most circumstances, described below. Two attempts are made to find a displaced vertex: the first (known as PASS 1) demands at least three tracks form the vertex. This pass has rather loose selection criteria; the second (PASS 2) will accept two-track vertices, but with more stringent selection criteria. PASS 2 is only attempted if PASS 1 fails to find an acceptable seed vertex.

If either PASS 1 or PASS 2 succeeds, a standard set of quantities is calculated and made available to the user. These include: the two-dimensional transverse decay length $\left(L_{x y}\right)$, the vertex displacement projected onto the jet $\operatorname{axis}^{2}$; the uncertainty on $L_{x y}$; the invariant mass of the tracks in the vertex $\left(M_{c l}\right)$, assuming each track has the pion mass; and the $\chi^{2}$ of the vertex fit. Once a displaced vertex has been found, cuts can be placed on these quantities to improve the $b$ purity of tagged events.

The secondary vertex tagging procedure is summarized in Figure 4.3; it is a useful reference for the discussion that follows.

\footnotetext{
${ }^{2}$ The jet axis, or direction, is defined by a vector from the CDF origin to the energy-weighted average position of the calorimeter towers included in the jet.
} 
START

\begin{tabular}{|c|} 
associate $\mathrm{SVX}$ \\
tracks to jet if \\
within a cone of \\
0.7 of jet axis
\end{tabular}$\rightarrow \rightarrow$\begin{tabular}{c|} 
flag tracks \\
surviving \\
PASS 1 cuts \\
as "displaced"
\end{tabular}$\rightarrow \rightarrow \begin{gathered}\text { order displaced } \\
\text { tracks by } \\
\text { Ngood, } \mathrm{P}_{\mathrm{t}}, \\
\text { and } \mathrm{S}_{\mathrm{d} 0}\end{gathered}$

PASS 1

take tracks from ordered list pairwise and try to form a seed vertex. One track in the vertex must have

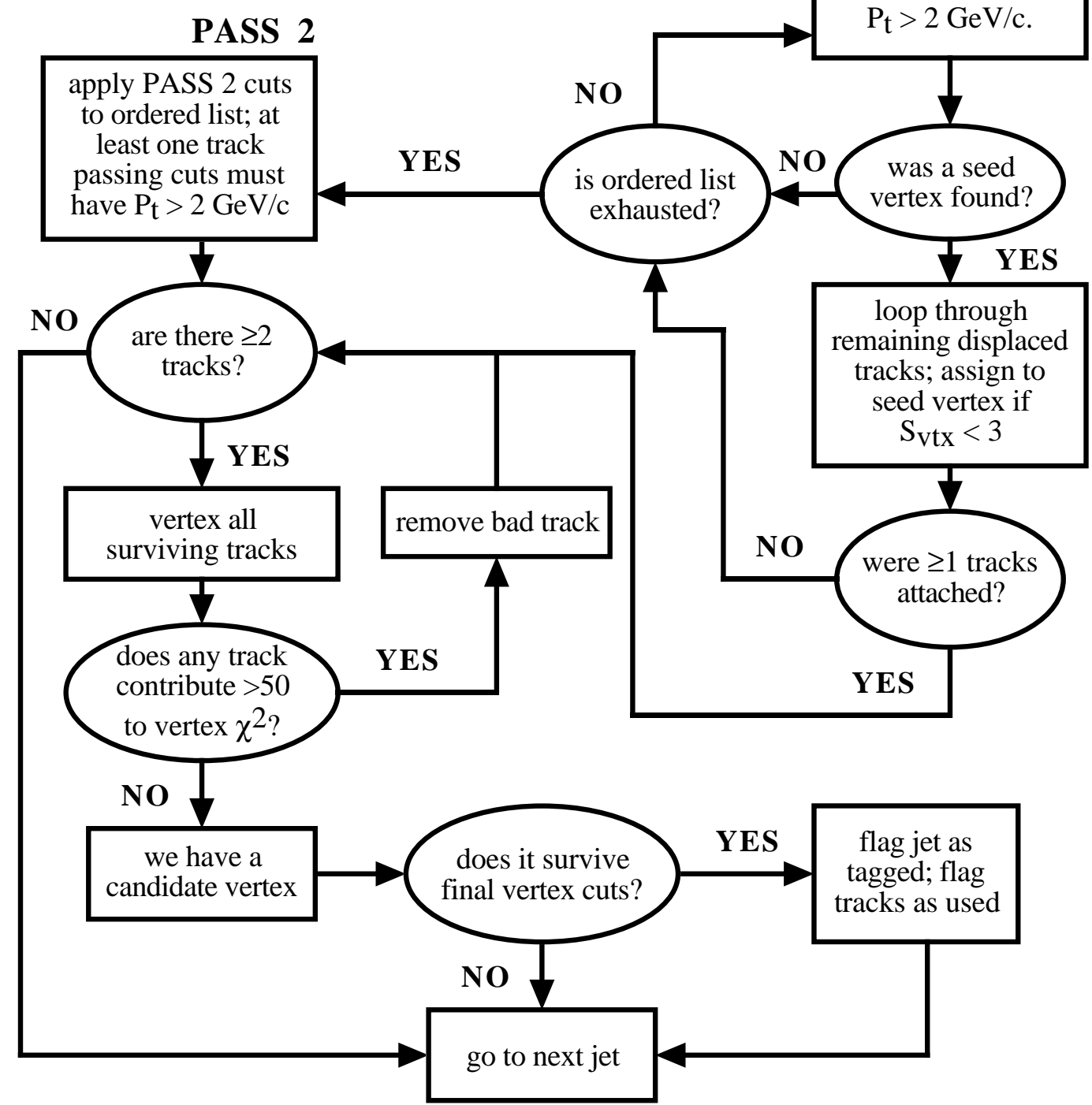

Figure 4.3: A flowchart summarizing the SECVTX algorithm described in Section 4.3. 


\subsubsection{Jet Selection}

The default behavior of SECVTX is to consider jets which have been clustered in a cone ${ }^{3}$ of $\Delta R=0.4$. This is appropriate for the highly-energetic (therefore narrow) $b$ jets from top decay, but less so for the softer inclusive lepton events. A theoretical calculation of direct $b \bar{b}$ production indicates that, on average, at least $90 \%$ of the $b$ energy is contained within a cone of 0.7 ; the jet clusters of this size are used here.

To be considered by SECVTX, jets must have $\mathrm{E}_{\mathrm{t}}>10 \mathrm{GeV}$ and $|\eta|<2$. This requirement creates a kinematic difference between the trigger electron and muon samples; electrons deposit all of their energy into the calorimeter, while muons do not. Consequently, both the $b$ quark energy and track multiplicity will, on average, be larger in muon events.

\subsubsection{Track Preselection}

SVX tracks must first be assigned to jets, as SECVTX attempts to construct tags in each jet. To be considered in a jet, a track must be within $5 \mathrm{~cm}$ in $z$ of the primary interaction vertex and have an impact parameter $(D)$, relative to the primary vertex, of less than $1.5 \mathrm{~mm}$. While a large impact parameter is more consistent with a displaced vertex than the primary, too large an impact parameter can indicate that the track is poorly measured, a result of pattern recognition error, or has an origin in the material of the detector (such as protons knocked out of the beampipe). Tracks meeting these requirements which are within a cone of $\Delta R=0.7$ of a particular jet axis are assigned to that jet.

Further cuts are applied to all SVX tracks to select those which may be considered for PASS 1 or 2 tagging. The cuts are summarized in Table 4.2. Tracks

\footnotetext{
${ }^{3}$ Recall that $(\Delta R)^{2}=(\Delta \eta)^{2}+(\Delta \phi)^{2}$.
} 


$$
\begin{aligned}
& p_{\mathrm{t}}>0.5 \mathrm{GeV} / \mathrm{c} \\
& \left|z-z_{\text {primary }}\right|<5 \mathrm{~cm} \\
& |D|<0.15 \mathrm{~cm} \\
& \text { pass standard CTC track quality requirements } \\
& \chi^{2} / \text { dof }<6 \\
& \mathrm{~N}_{\text {good }} \geq 1 \\
& \text { not used in a } K_{S}^{0} \text { or } \Lambda^{0} \text { "vee" } \\
& \text { not already used in another tag in this event }
\end{aligned}
$$

Table 4.2: SECVTX SVX track preselection requirements. $\mathrm{N}_{\text {good }}$ (the number of good SVX clusters), the CTC track quality cuts, and the $K_{S}^{0} / \Lambda^{0}$ vertex cuts are defined in the text.

must have $p_{\mathrm{t}}>0.5 \mathrm{GeV} / \mathrm{c}$, a good SVX-CTC matching $\chi^{2}$, at least one good SVX cluster, and must pass standard quality requirements. Tracks may not be suspected of being products from the decay of a $K_{S}^{0}$ or a $\Lambda^{0}$. Further discussion of these cuts follows:

\section{Good SVX Clusters}

An SVX cluster (a set of contiguous strips with charge above threshold; refer to Section 2.2.1) is considered "good" if it is $\leq 3$ strips long, contains no noisy or dead strips, and is not "shared." Sharing is when the same cluster is used by more than one SVX track, typically in cases of energetic jets, where particles can be very dense in $\phi$. All tracks considered for tagging must have a minimum of two clusters, at least one of which must be a good cluster.

\section{CTC Track Requirements}

As discussed earlier in Section 2.2.1, loose cuts on the CTC portion of a track's information can greatly reduce the chance that the track was actually generated by a failure in the pattern-recognition software. SECVTX requires, in almost all cases, that each track meet the standard set of CTC quality requirements: the track

must have $z$ information (from a successful three-dimensional fit), two or more axial 
superlayers with four or more hits, and two or more stereo superlayers with two or more hits.

\section{$K_{S}^{0}$ and $\Lambda^{0}$ Removal}

Care is taken to ensure that SECVTX does not find a $b$-tag from a $K_{S}^{0}$ or a $\Lambda^{0}$ (colloquially called vees), which are long-lived enough to result in a displaced-vertex tag, but are not necessarily themselves the decay products of heavy flavor. Before any tagging attempts are made, every SVX track with $\left|D / \sigma_{D}\right|>2.5$ is paired with every oppositely-charged SVX track meeting the same requirement. The large $\left|D / \sigma_{D}\right|$ indicates that each track is most likely not consistent with an origin at the primary. One track of the pair must pass the CTC cuts, but no requirements on the quality of the SVX tracks or the tracks' $p_{\mathrm{t}}$ are made. The CTC cuts are not imposed on both tracks as the goal is to maximize the efficiency for finding vees so they may be eliminated; the soft pion from $\Lambda^{0} \rightarrow \mathrm{p} \pi$ is often hard to reconstruct and can result in a very low-quality track. Requiring that one track passes the CTC cuts saves some computational time, as if neither do, then neither will be considered for candidate tracks in a tag vertex, and it is then irrelevant whether they come from a vee or not.

The pair is constrained to come from a common point by a steered vertexconstrained fit ${ }^{4}$, allowing a calculation of the vector displacement of the point from the primary vertex. The "vee axis" is defined to be the vector sum of the steered momenta of the two tracks. If the displacement divided by its uncertainty (the displacement significance), both projected along the vee axis, is greater than 10 , and the displacement significance perpendicular to the vee axis is less than 3 , then

\footnotetext{
${ }^{4}$ Recall that a steered fit is one in which the track parameters can be adjusted, within their uncertainties, to minimize the $\chi^{2}$ of the fit.
} 


\begin{tabular}{|c|}
\hline $\begin{array}{l}\text { meet SVX track preselection requirements } \\
\text { not already used in another tag in this event } \\
\left|D / \sigma_{D}\right|>2.5\end{array}$ \\
\hline $\begin{array}{l}\text { for two-hit SVX tracks: } \\
\end{array}$ \\
\hline $\begin{array}{l}p_{\mathrm{t}}>1.5 \mathrm{GeV} / \mathrm{c} \\
\mathrm{N}_{\text {good }}=2 \\
\text { hit pattern } 1100 \text { or } 0011\end{array}$ \\
\hline
\end{tabular}

Table 4.3: SECVTX PASS 1 SVX track requirements. The preselection cuts are listed in Table 4.2. $\mathrm{N}_{\text {good }}$ (the number of good SVX clusters) is defined in the text. The hit pattern indicates on which layers of the SVX the clusters are found; for two-hit SVX tracks, the PASS 1 requirement is that the clusters must be on the two outermost or two innermost layers.

the pair is consistent with the decay products of a neutral particle coming from the primary vertex, and the invariant mass of the pair is calculated using the steered momenta. If the vertex mass is within $10 \mathrm{MeV} / \mathrm{c}^{2}$ of the $K_{S}^{0}$ mass or within 6 $\mathrm{MeV} / \mathrm{c}^{2}$ of the $\Lambda^{0}$ mass, both tracks are flagged as "vee candidates," and are not considered for any $b$-tagging by SECVTX.

\subsubsection{PASS 1 Tagging}

A subset of the preselected tracks is prepared by subjecting each track to additional cuts, summarized in Table 4.3. The key cut is the requirement that $\left|D / \sigma_{D}\right|>2.5$, indicating that the track is unlikely to have originated at the primary; recall that the uncertainty on $D$ includes a contribution from the uncertainty on the primary vertex. Tracks which have been included in a previous tag (in another jet) are excluded. The additional cuts on two-hit SVX tracks improve their quality, though there are few of them to start with ${ }^{5}$. The subset of preselected tracks passing these cuts are known as the "PASS 1" tracks.

\footnotetext{
${ }^{5}$ Approximately $8.3 \%$ of low- $p_{\mathrm{t}}$ SVX-fiducial tracks have only two hits.
} 


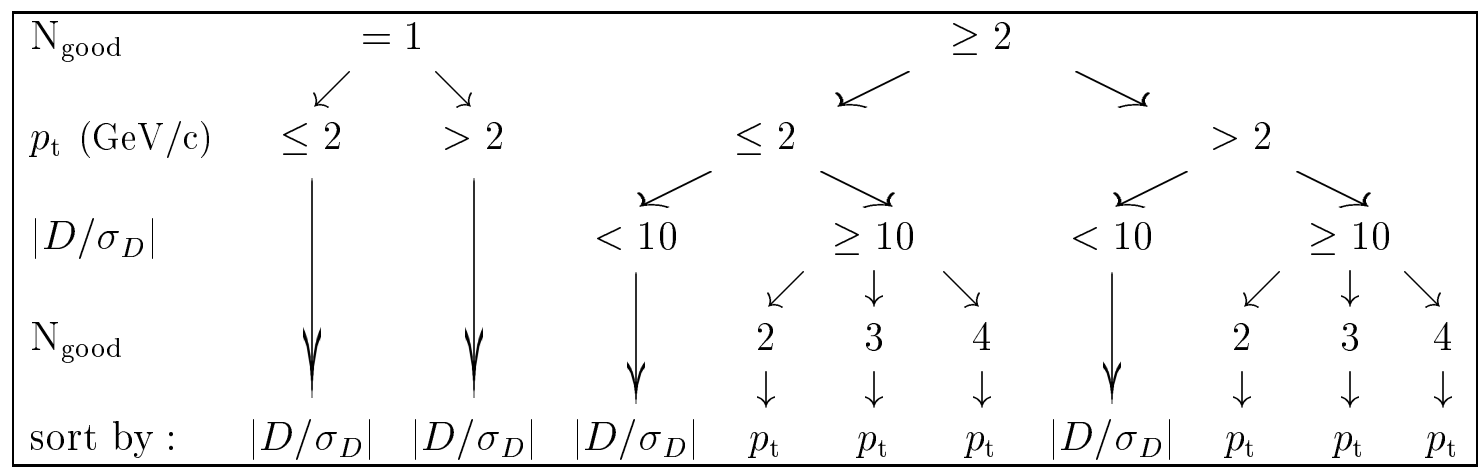

Figure 4.4: SECVTX SVX track-ordering flowchart. For each track meeting the PASS 1 requirements, the flowchart is followed based on the track's $\mathrm{N}_{\text {good }}, p_{\mathrm{t}}$, and $\left|D / \sigma_{D}\right|$ until one of the bottom 10 bins is reached. Tracks in each bin are sorted by the specified key. Rightmost bins are ordered first.

\section{SVX Track Sorting}

The PASS 1 algorithm is based on finding a seed vertex with two tracks, and determining if any other tracks have a low impact parameter significance relative to the seed $\left(S_{v t x}\right)$. Instead of finding all possible seeds, PASS 1 pairs tracks in order of their quality. The order is based on the $\mathrm{N}_{\text {good }}$ (the number of good SVX clusters), $p_{\mathrm{t}}$, and $\left|D / \sigma_{D}\right|$ of each track; the sort algorithm is shown in Figure 4.4. In general, tracks with more good SVX clusters, higher $p_{\mathrm{t}}$, and larger $\left|D / \sigma_{D}\right|$ are "better".

\section{PASS 1 Seed Vertexing}

Pairs of PASS 1 tracks are looped over, in order, and constrained to come from a common vertex. One member of each pair must have $p_{\mathrm{t}}>2 \mathrm{GeV} / \mathrm{c}$. Should the fit of the first and second tracks fail, the first track is paired with the third, then the fourth, and so on, until a successful fit is found. $S_{v t x}$ is calculated for each remaining track, and those with $S_{v t x}<3$ are considered associated with the seed — others are subsequently ignored. In this usage, "remaining" refers to the tracks which are ordered after the two tracks in the seed; for example, if the first and third tracks are used to form the seed, then the remaining tracks are the fourth through 
last. The second can be ignored as it obviously failed to form a two-track vertex with the first.

A seed vertex with at least one additional track in association defines a potential three-track tag vertex. If such a potential tag is found, the loop over pairs of tracks terminates, PASS 2 tagging is not attempted, and the two tracks in the seed plus all associated tracks are passed to the candidate vertexing algorithm, defined in Section 4.3.6.

\subsubsection{PASS 2 Tagging}

PASS 2 tagging searches for two-track displaced vertices. As the smaller number of tracks allows for a larger combinatoric background, the cuts on the individual tracks are tightened. The requirements are described in Table 4.4. It is worth repeating that PASS 2 tagging will proceed only if no potential PASS 1 tag is found; this is more restrictive than the rule of attempting PASS 2 if PASS 1 fails for any reason.

No seed vertexing is performed by PASS 2. One of the tracks passing the requirements must have $p_{\mathrm{t}}>2.0 \mathrm{GeV} / \mathrm{c}$, and there most be at least two tracks in total. All tracks are passed collectively to the candidate vertexing algorithm.

\begin{tabular}{|l|}
\hline meet PASS 1 requirements \\
$\left|D / \sigma_{D}\right|>3.0$ \\
$p_{\mathrm{t}}>1.0 \mathrm{GeV} / \mathrm{c}$ \\
two-hit SVX tracks not allowed \\
\hline \hline for three-hit SVX tracks: \\
\hline $\mathrm{N}_{\text {good }} \geq 2$ \\
\hline
\end{tabular}

Table 4.4: SECVTX PASS 2 SVX track requirements. $\mathrm{N}_{\text {good }}$ (the number of good SVX clusters) is defined in the text; the PASS 1 cuts are listed in Table 4.3. 


\subsubsection{Candidate Vertexing}

Given a list of tracks from either PASS 1 or PASS 2, SECVTX attempts to constrain all tracks to come from the same vertex. Should the fit succeed, the track which contributes the most to the vertex $\chi^{2}$ is rejected if its contribution is more than 50. If a track is rejected, the vertex fit is performed again, and the procedure repeated until no more tracks are removed, or the number of tracks falls below two. If at any time, the vertex fit fails for any reason (i.e. the tracks don't actually originate at a common vertex), the PASS 1 or 2 vertexing attempt fails and is immediately stopped. While this track rejection algorithm may suggest that it is possible to obtain a two-track PASS $1 \mathrm{tag}$, in practice, the requirement that associated tracks have $S_{v t x}<3$ results in a practical impossibility that they can contribute more than 50 to the $\chi^{2}$ of a successful vertex fit.

Given a successful vertex, cuts are applied to ensure that PASS 1 or 2 has not re-found the primary vertex through this laborious route, but rather, found a true secondary, displaced vertex. Additionally, the secondary must not be too displaced; $b$ hadrons are not so long-lived compared to $K_{S}^{0}$ s and $\Lambda^{0}$ s. In addition, "fake" tracks from pattern recognition errors can have very large impact parameters (and therefore preferentially selected by the PASS 1 and 2 cuts), resulting in highlydisplaced vertices having nothing to do with a real heavy-flavor decay. Finally, $K_{S}^{0} \mathrm{~s}$ are once more screened out if the vertex consists of only two oppositely-charged tracks. This step is necessary as the vetoing of "vee tracks" in the preselection requirements only rejects those track pairs with displacement significances much larger than what SECVTX will now accept, as described below.

The two-dimensional decay length, $L_{x y}$, is calculated by taking the dot product of the displacement vector (the secondary vertex position minus the primary position) and a unit vector along the jet axis. $L_{x y}$ is therefore a signed quantity: positive if 
the displacement is in the same direction as the jet, negative if it is away from the jet. The error on $L_{x y}\left(\sigma_{L_{x y}}\right)$ is calculated in a similar fashion: it is the addition in quadrature of the projection of the uncertainty ellipses of the primary and secondary vertices along the jet axis. The cluster momentum is given from the vector sum of the tracks' momenta. The tag mass $\left(M_{c l}\right)$ is defined as the invariant mass of the tracks forming the secondary vertex, assuming each has the pion mass. An estimate of the proper lifetime $(\mathrm{c} \tau)$ of the vertex is calculated from the decay length, mass, and $p_{\mathrm{t}}$ of the cluster: $\mathrm{c} \tau=L_{x y} \cdot M_{c l} / p_{\mathrm{t}}$. The cuts for an acceptable displaced vertex are listed in Table 4.5. The absolute cuts on $L_{x y}$ and $\mathrm{c} \tau$ remove the high-side tail from fake tracks and the cut on the displacement significance $\frac{\left|L_{x y}\right|}{\sigma_{L_{x y}}}$ ensures that the secondary vertex is unlikely to actually be the primary found with fewer tracks. It should be noted that the estimate of $\mathrm{c} \tau$ calculated by SECVTX is used nowhere else in this analysis - a more accurate estimate will be derived in Section 5.2.

If a successful tag is found, the jet is marked as "tagged," the tracks in the tag are marked as "used," tagging attempts in this jet cease, and SECVTX proceeds to the next jet.

$$
\begin{aligned}
& \left|L_{x y}\right|<2.5 \mathrm{~cm} \\
& |\mathrm{c} \tau|<1 \mathrm{~cm} \\
& \frac{\left|L_{x y}\right|}{\sigma_{L_{x y}}} \geq 2 \\
& \left|M_{c l}-M_{K_{S}^{0}}\right|>20 \mathrm{MeV} / \mathrm{c}^{2}
\end{aligned}
$$

Table 4.5: SECVTX secondary vertex requirements. The cut on the tag mass is only applied if the number of tracks in the tag is two, and they are of opposite charge. 


\subsection{Event Selection Using SECVTX}

Given the excellent primary and secondary vertex-finding capabilities of VXPRIM and SECVTX, they can be used in conjunction to select events consistent with $b \bar{b}$ production from the primary datasets.

The requirement that there be a SECVTX displaced-vertex tag in the same jet as the highest- $p_{\mathrm{t}}$ trigger electron in the event is imposed on the 2,626,500 events in the primary inclusive electron dataset. Cross-identification of electron to jet is easily done. As electron candidates are first found by clusters of electromagnetic energy, every electron is itself a jet. Production includes a link to the jet information in the electron candidate information. 89,707 inclusive electron events meet this SECVTX requirement. Approximately $86 \%$ of these events have the electron in the tag: that is, the electron track is one of the tracks vertexed in the SECVTX displaced-vertex.

In the 2,009,040 events in the primary inclusive muon dataset, a SECVTX tag is required in the same jet as any trigger muon, not just the one with the highest- $p_{\mathrm{t}}$, allowing for a slightly higher percentage of accepted events than for trigger electrons, for the cases where there is more than one trigger lepton in the event. In addition, the muon-jet cross-link must be accomplished differently, since the muon candidate algorithm is track-based, not calorimeter-based. The identification is established by searching for the muon track on the list of tracks in jets prepared by SECVTX. This amounts to a requirement that the muon has an SVX track, as SECVTX only considers SVX tracks. 82,679 inclusive muon events meet the requirement that a trigger muon be in a SECVTX-tagged jet, of which approximately $80 \%$ have the muon as a member of the tag.

The differences in event selection between the electron and muon samples have only a minor effect. $99.5 \%$ of trigger muons in tagged jets are the highest- $p_{\mathrm{t}}$ muon 
in the event and $99.6 \%$ of trigger electrons have SVX tracks. Both results are expected. The SECVTX tagging efficiency rises with $p_{\mathrm{t}}$. Trigger electrons will be within $5 \mathrm{~cm}$ of the SECVTX tag tracks, and thus very likely to be in the SVX' acceptance.

\subsection{Track-Clustered Jet Definition (TRKSJT)}

An alternative to jets defined by clusters of energy in the calorimeter is jets defined by "clusters" of tracks located closely together. As track-clustered jets will be used in the final sample requirements (described in Section 4.6), as well as in the remainder of this analysis ${ }^{6}$, the clustering algorithm is described here. An advantage of track-clustered jets is that the jet direction is more precisely determined by use of tracking information; a disadvantage is that neutral energy (in the form of photons or $\pi^{0} \mathrm{~s}$ ) is not included. Despite this disadvantage, track-clustered jets well-match the underlying $b$ quark direction.

This analysis uses the TRKSJT track-clustering software package [58], which is entirely based on CTC tracks. A cluster is defined as a group of tracks, close in $z$, within a specified cone $(\Delta R)$ in $\eta$ - $\phi$ coordinates. Two cone sizes are used: $\Delta R=0.4$ and $\Delta R=0.8$. All tracks considered in the clustering must have $p_{\mathrm{t}}>0.4 \mathrm{GeV} / \mathrm{c}$ and an impact parameter (relative to the CDF origin) of less than $5 \mathrm{~cm}$. They must also be within $5 \mathrm{~cm}$ of the primary vertex and pass the standard quality cuts on the number of hits in axial and stereo superlayers. Tracks with $p_{\mathrm{t}}>1.0 \mathrm{GeV} / \mathrm{c}$ are considered as the "seeds" of the tracking jets; those closer together than the cone size are merged, that is, the seed-jet is taken to be the vector sum of the track

\footnotetext{
${ }^{6}$ Chapter 6 shows how the composition of the samples can be determined from the $p_{\mathrm{t}}^{\text {rel }}$ of the trigger lepton relative to its parent $b$ jet, and Chapter 7 describes how a weighted charge average of a jet's constituent tracks (the jet charge) is used to tag the flavor of the second $b$ in each event.
} 
momenta. After the merging, all tracks must lie within the cone of the seed-jet; otherwise, the merge result is rejected. When no more seed tracks can be merged, all seed-jets are farther than $\Delta R$ from each other. The remaining tracks are then merged into the closest seed-jet (in $\eta$ - $\phi$ coordinates), subject to the condition that they are within $\Delta R$ of the closest jet.

\subsection{Additional Sample Requirements}

A few additional cuts are placed on the samples, to ensure their quality and prepare them for further studies. The trigger lepton must pass the standard track quality cuts, be within $5 \mathrm{~cm}$ in $z$ of the primary vertex, and be a member of a TRKSJT track-clustered jet. The requirements have only a small inefficiency (largely from the $|\Delta z|$ cut); they reduce the sample sizes to 89,262 trigger electron events and 82,656 trigger muon events. 


\section{Chapter 5}

\section{Monte Carlo Samples and Studies}

Monte Carlo simulation is an important tool in modern high-energy experimental particle physics. The Standard Model is deceptively simple - the calculation of many physical processes involve integrations with so many degrees of freedom that numerical interpolation methods are too time-consuming to consider their use. In addition, what is desired is not just the theoretical description of a particular process, but rather, how events from this process would appear in a physical detector, with the associated geometric acceptances and resolution effects. Monte Carlo simulations answer both concerns. In common usage, the name refers both to the calculation of intractable integrals through random sampling and to the simulation of detectors, based upon measured performance.

This chapter documents the preparation of the simulated $b \bar{b}$ and $c \bar{c}$ samples, which are used to improve the understanding of the data samples in many ways. A critical component of any time-dependent mixing measurement is the proper time $(\mathrm{c} \tau)$ at which the $b$ hadron decayed. $\mathrm{c} \tau$ can be estimated from information provided by SECVTX: the two-dimensional decay distance $\left(L_{x y}\right)$ of the secondary vertex and the tag tracks' $p_{\mathrm{t}}$ and invariant mass. The $b \bar{b}$ Monte Carlo samples allow a study 
of the resolution and bias of such an estimation. The impact parameter and $L_{x y}$ significance requirements of SECVTX result in it preferentially selecting events with a longer lifetime. The SECVTX tagging efficiency as a function of $\mathrm{c} \tau$ must be determined, to compensate for this bias in the fit for $\Delta m_{d}$. The correlation of the charge of trigger leptons with the $b$ flavor must also be understood. The $b \bar{b}$ Monte Carlo samples are used to study the fraction of trigger leptons from the direct and various sequential $b$ decay modes. Following chapters use the Monte Carlo samples to determine the fraction of $b \bar{b}$ and $c \bar{c}$ events in the data samples and study the effectiveness of flavor-tagging under different conditions.

\subsection{Monte Carlo Sample Generation}

The samples are designed to model leptons from $b \bar{b}$ and $c \bar{c}$ production and decay. The program PYTHIA [59] is commonly used to simulate multiparticle production in collider environments. Here, PYTHIA (version 5.6) is used to simulate $b \bar{b}$ and $c \bar{c}$ production in $p \bar{p}$ collisions. The program QQ [60] (version 9.1) is used to decay charmed and $b$ hadrons.

For the $b \bar{b}$ generation, the collision must have a minimum momentum transfer $\hat{p_{\mathrm{t}}}>8 \mathrm{GeV} / \mathrm{c}$. Mixing of $B_{d}^{0}$ and $B_{s}^{0}$ mesons is allowed, with $x_{d}=0.71$ and $x_{s}=10.0$, respectively. The full default $\mathbf{Q Q}$ decay table is used for the decay of $b$ and charmed hadrons, to ensure that the fraction of trigger leptons from sequential decays is correct. For $c \bar{c}$ generation, the minimum $\hat{p}_{\mathrm{t}}$ is changed ${ }^{1}$ to $15 \mathrm{GeV} / \mathrm{c}$, and the charmed hadrons are forced to decay semileptonically to increase the generation efficiency. This alters the ratio of trigger leptons from $D^{0}$ versus $D^{+}$(and thus the

\footnotetext{
${ }^{1}$ As charm fragmentation is softer than $b$ fragmentation, charmed mesons from direct $c \bar{c}$ production will, on average, carry a lower fraction of the jet $p_{\mathrm{t}}$. Consequently, a higher $\hat{p}_{\mathrm{t}}$ requirement can be imposed to improve the generation efficiency with no bias to the sample after all event selection requirements.
} 
average charm lifetime, since the lifetimes of the two are very different), as well as vastly improving the efficiency for finding a soft lepton from the other charm. The effect of the $D^{0}: D^{+}$ratio on the average lifetime is included in the calculation of the systematic error of the final result (see Section 8.2). For both $b \bar{b}$ and $c \bar{c}$ generation, the Peterson fragmentation parameters are set to $\epsilon_{b}=0.006$ and $\epsilon_{c}=$ 0.06 , respectively.

All generated leptons are first filtered through a simulation of the Level 2 CFT trigger, which in data-taking required the presence of a $7.5 \mathrm{GeV} / \mathrm{c}$ track. The parametrization of the trigger efficiency has the form

$$
E_{0} \cdot \text { freq }\left[\frac{p_{\mathrm{t}}-a_{1}}{a_{2}}\right] \cdot \text { freq }\left[\frac{p_{\mathrm{t}}-a_{3}}{a_{4}}\right]
$$

where

$$
E_{0}=0.927, \quad a_{1}=6.18, \quad a_{2}=4.20, \quad a_{3}=7.48, \quad a_{4}=0.504
$$

and freq is the error function. $E_{0}$ is the plateau CFT efficiency; $a_{3}$ and $a_{4}$ dominate the trigger turn-on, at $7.5 \mathrm{GeV} / \mathrm{c}$. Only those events which have a lepton passing the Level 2 simulation are kept.

Those events are then processed through a simulation of the CDF detector, which includes a detailed strip-by-strip simulation of $\mathrm{SVX}^{\prime}$ [61]. The simulation also includes a tuning of the CTC wire hit efficiencies to better match the data [62]. Finally, all simulated events are required to pass the same event selection as the final data samples; that is, identifying one or more trigger leptons, finding the primary vertex with VXPRIM, finding a SECVTX tag in the same jet as a trigger lepton (including the difference between the electron and muon data sample selection), and applying the remaining quality cuts. After all requirements are met, the $b \bar{b}$ samples comprise 51,884 trigger electron events and 56,104 trigger muon events, and the $c \bar{c}$ 
samples comprise 18,368 trigger electron events and 21,643 trigger muon events.

\subsection{Proper Decay Time $(c \tau)$ Determination}

The proper time can be calculated from the two-dimensional decay distance $\left(L_{x y}\right)$, supplied by the SECVTX tag in the trigger lepton jet, with the equation

$$
\mathrm{c} \tau=\frac{L_{x y} \cdot m_{B}}{p_{\mathrm{t}}^{B}}
$$

The $b$ hadron mass shall be taken from the PDG world-average value; all that remains is to calculate the hadron's $p_{\mathrm{t}}$. An exact determination cannot be made, as the semileptonic decay cannot be fully-reconstructed; it includes a neutrino, whose energy escapes from the detector. Therefore, $p_{\mathrm{t}}^{B}$ must be estimated, using available observables. The Monte Carlo samples are relied upon to verify the measurement of $L_{x y}$ and the estimation of $p_{\mathrm{t}}^{B}$.

The difference between $L_{x y}$ as reconstructed by SECVTX and the generated, or true, $L_{x y}$ for the muon $b \bar{b}$ Monte Carlo is shown in Figure 5.1. The mean of the distribution is clearly positive, indicating that SECVTX tends to overestimate the true $L_{x y}$. While unfortunate, this is expected, as charmed hadrons produced in $b$ decay have a lifetime of their own. Some fraction of the time, SECVTX will use tracks from the charm decay to identify the secondary vertex. The distribution is fit to three Gaussians; the central Gaussian, with a mean of $141 \mu \mathrm{m}$, accounts for $67 \%$ of the events. This mean is taken as the bias on $L_{x y}\left(\delta L_{x y}\right)$. A study of the electron $b \bar{b}$ sample finds a $\delta L_{x y}$ of $135 \mu \mathrm{m}$. The bias will now be subtracted from the reconstructed $L_{x y}$ in this and all remaining chapters. The contribution of $\delta L_{x y}$ to the systematic uncertainty of the $\Delta m_{d}$ measurement will be evaluated in Chapter 8 by varying the scale of $\delta L_{x y}$ from zero to twice its value. 


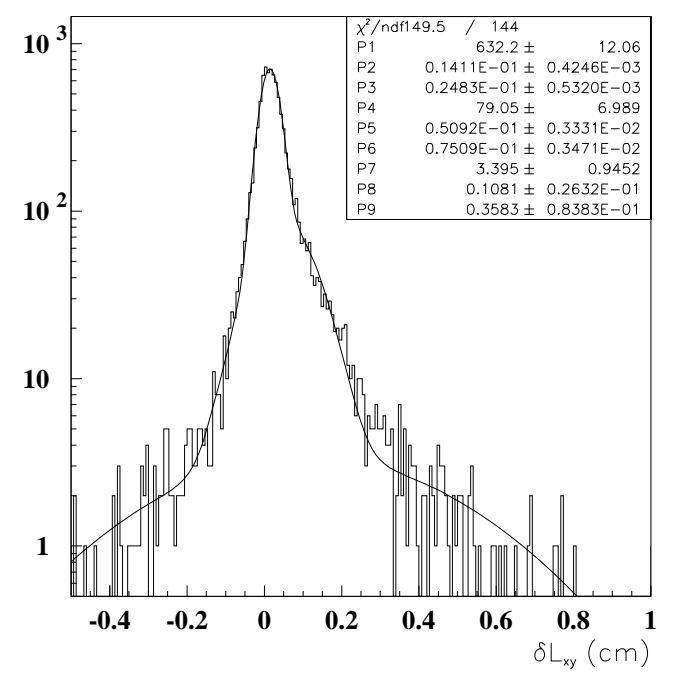

Figure 5.1: The distribution of $\delta L_{x y}$, the difference between reconstructed $L_{x y}$ and true $L_{x y}$, for the muon $b \bar{b}$ Monte Carlo sample. The distribution is fit to the sum of three Gaussians; the fit parameters, indicated above, are three sets of amplitude, mean, and width (in that order).

The method used by this analysis to estimate $p_{\mathrm{t}}^{B}$ is to use the total $p_{\mathrm{t}}$ of the tracks in the vertex tag as a first estimate, then derive a correction factor from the $b \bar{b}$ Monte Carlo. This track-based method implicitly ignores energy from other neutral particles (such as $\pi^{0} \mathrm{~s}$ ) in addition to the neutrino, as well as those tracks which fail to meet the tag requirements of SECVTX. It will be demonstrated that the correction can overcome these apparent shortcomings.

The correction factor is derived from the SECVTX tag tracks' $p_{\mathrm{t}}$ and invariant mass $\left(p_{\mathrm{t}}^{c l}\right.$ and $\left.M_{c l}\right)$. Essentially, it is the ratio $p_{\mathrm{t}}^{c l} / p_{\mathrm{t}}^{B}$, calculable because the Monte Carlo simulation allows the inspection of the true $p_{\mathrm{t}}^{B}$. In the minority of cases where the trigger lepton is not a member of the tag, it is included in the calculation of $p_{\mathrm{t}}^{c l}$ and $M_{c l}$, as the lepton is expected to carry a significant portion of $p_{\mathrm{t}}^{B}$, even if its $\left|D / \sigma_{D}\right|$ is too small to pass the SECVTX requirements. Kinematic differences between the electron and muon triggers necessitate separate correction factors; to minimize any bias, separate factors are also calculated for the cases where the trigger lepton is or is not a tag track. The distribution of $p_{\mathrm{t}}^{c l} / p_{\mathrm{t}}^{B}$ is different for different 


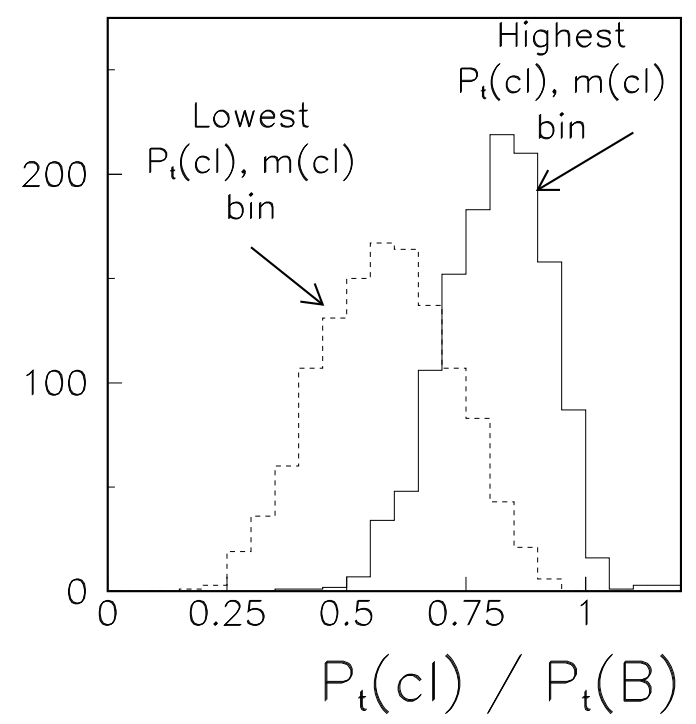

Figure 5.2: The observed $p_{\mathrm{t}}^{c l} / p_{\mathrm{t}}^{B}$ fraction for the highest and lowest $p_{\mathrm{t}}^{c l}, M_{c l}$ bins. The plots are from the electron $b \bar{b}$ Monte Carlo for events where the electron is a SECVTX tag track.

values of $p_{\mathrm{t}}^{c l}$ and $M_{c l}$, so the Monte Carlo samples are divided into 4 bins of $p_{\mathrm{t}}^{c l}$ and 4 bins of $M_{c l}$, where the number of bins are chosen to retain sufficient statistics and the bin boundaries are chosen to give equal statistics in each bin. There are therefore 64 different $p_{\mathrm{t}}^{c l} / p_{\mathrm{t}}^{B}$ distributions in the calculation of the correction "factor" $(2 \times 2 \times 4 \times 4=64)$.

Figure 5.2 shows the $p_{\mathrm{t}}^{c l} / p_{\mathrm{t}}^{B}$ distributions for the highest and lowest $p_{\mathrm{t}}^{c l}, M_{c l}$ bins, for the trigger electron $b \bar{b}$ sample, requiring the electron to be a tag track. Events in the highest bin clearly have the SECVTX tag tracks carrying, on average, a higher fraction of the $b$ hadron $p_{\mathrm{t}}$ than do those in the lowest bin; note also that the width of the distributions is narrower for the highest bin, indicating that the correction yields a better resolution. The resolution is a concern, because in the fit for $\Delta m_{d}$, the theoretical mixing probability density (a function of the true proper time) must be convoluted with the experimental $\mathrm{c} \tau$ resolution, which is itself a convolution of the resolutions on $L_{x y}$ and $p_{\mathrm{t}}^{B}$.

Two techniques can be used to estimate $p_{\mathrm{t}}^{B}$ from $p_{\mathrm{t}}^{c l}$. The most straightforward 
is to use the mean of the appropriate $p_{\mathrm{t}}^{c l} / p_{\mathrm{t}}^{B}$ distribution, accordingly:

$$
p_{\mathrm{t}}^{\prime}=\frac{p_{\mathrm{t}}^{c l}}{\left\langle p_{\mathrm{t}}^{c l} / p_{\mathrm{t}}^{B}\right\rangle_{i j}}
$$

where $p_{\mathrm{t}}^{\prime}$ is the estimate of $p_{\mathrm{t}}^{B}$ and $i j$ denotes the $i^{\text {th }} p_{\mathrm{t}}^{c l}$ bin and the $j^{\text {th }} M_{c l}$ bin. The fractional resolution on $p_{\mathrm{t}}^{\prime}$ in each bin can be estimated from the RMS width of the $p_{\mathrm{t}}^{c l} / p_{\mathrm{t}}^{B}$ distributions:

$$
\sigma_{p_{\mathrm{t}}^{\prime}}(\%)_{i j}=\frac{\operatorname{RMS}\left(p_{\mathrm{t}}^{c l} / p_{\mathrm{t}}^{B}\right)_{i j}}{\left\langle p_{\mathrm{t}}^{c l} / p_{\mathrm{t}}^{B}\right\rangle_{i j}}
$$

$\sigma_{p_{\mathrm{t}}^{\prime}}(\%)_{i j}$ ranges roughly from $15 \%$ to $30 \%$ for the various bins. The drawback to this method for estimating $p_{\mathrm{t}}^{B}$ can be seen in Figure 5.3. The mean correction changes sharply between $p_{\mathrm{t}}^{c l}, M_{c l}$ bins, producing "spikes" in the $p_{\mathrm{t}}^{\prime}$ distribution.

The second method is to produce a distribution of each event's possible $p_{\mathrm{t}}^{\prime}$ (the estimate of $p_{\mathrm{t}}^{B}$ ) through weighting by the probability of each $p_{\mathrm{t}}^{c l} / p_{\mathrm{t}}^{B}$ in the appropriate $p_{\mathrm{t}}^{c l}, M_{c l}$ bin. This is the method used in the fit for $\Delta m_{d}$, and its superiority to the mean correction is also shown in Figure 5.3. The weighted-correction method represents a "smoothing" of the $p_{\mathrm{t}}^{\prime}$ distribution, and more accurately represents how the experimental $p_{\mathrm{t}}^{B}$ convolution is performed in the $\Delta m_{d}$ fit.

\subsection{Reconstructed $c \tau$ Efficiency}

SECVTX's track impact parameter requirements, necessary to ensure that the found secondary vertex is significantly displaced from the primary vertex, bias the lifetime distribution. SECVTX is rather inefficient for events with small $\mathrm{c} \tau$, owing to the minimum $\left|D / \sigma_{D}\right|$ cuts. Its efficiency will also fall, more slowly, with large $\mathrm{c} \tau$, due to the $D<1.5 \mathrm{~mm}$ cut. 

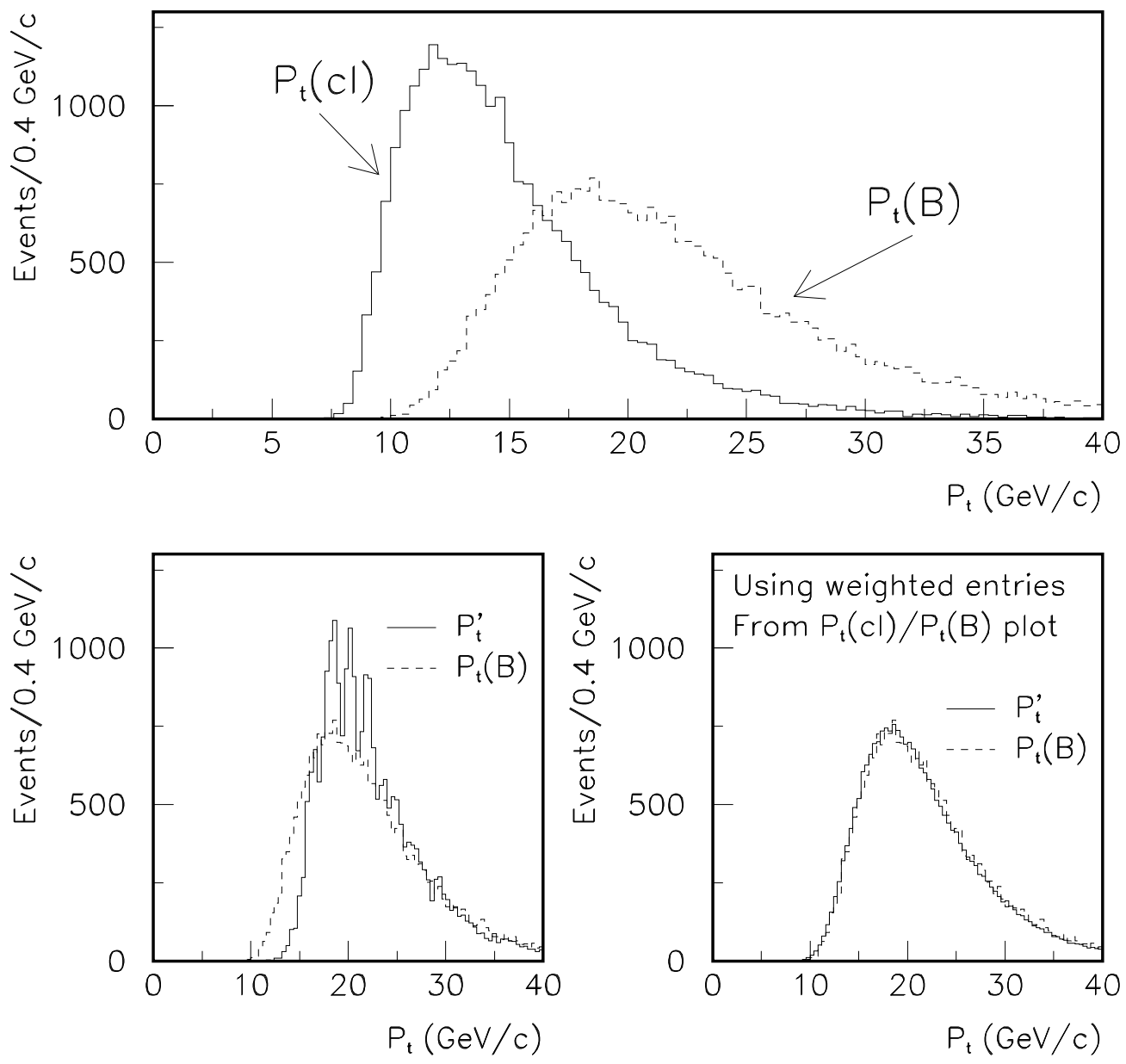

Figure 5.3: Top: The distributions of $p_{\mathrm{t}}^{c l}$ and true $p_{\mathrm{t}}^{B}$ for the $b \bar{b}$ electron Monte Carlo sample.

Lower left: Corrected $p_{\mathrm{t}}^{\prime}$ and true $p_{\mathrm{t}}^{B}$ distributions, where the correction is obtained from the mean of the $p_{\mathrm{t}}^{c l} / p_{\mathrm{t}}^{B}$ distributions.

Lower right: Corrected $p_{\mathrm{t}}^{\prime}$ and true $p_{\mathrm{t}}^{B}$ distributions, where the entire $p_{\mathrm{t}}^{c l} / p_{\mathrm{t}}^{B}$ distributions are used to make several weighted entries for each event. 
The efficiency as a function of $\mathrm{c} \tau$ must be understood so the predicted lifetime distribution can be properly compared with the measured distribution when fitting for $\Delta m_{d}$. The reconstructed $\mathrm{c} \tau$ distribution in the limit of perfect SECVTX c $\tau$ efficiency is modeled by a fast Monte Carlo (FMC) that uses the pre-SECVTX-tagging lifetime distributions from the Monte Carlo samples, and convolutes each event's generated $\mathrm{c} \tau$ with the measured resolutions from Section 5.2. The SECVTX efficiency is then the reconstructed $\mathrm{c} \tau$ distribution from the data divided by the same distribution from the FMC. This procedure is performed for trigger electron and muon samples separately. The distributions are shown in Figures 5.4 and 5.5. The FMC does not model the $\mathrm{c} \tau<0$ distribution well at all; only events with $\mathrm{c} \tau>0$ will be included in the fit for $\Delta m_{d}$.

The reconstructed $\mathrm{c} \tau$ efficiency, $\epsilon(\mathrm{c} \tau)$, is fit to the form

$$
\epsilon(\mathrm{c} \tau)=p_{0} \cdot\left[\operatorname{freq}\left(\mathrm{c} \tau \cdot p_{1}+p_{2}\right)+p_{3} \cdot \mathrm{c} \tau+p_{4} \cdot(\mathrm{c} \tau)^{2}+p_{5} \cdot(\mathrm{c} \tau)^{3}+p_{6} \cdot(\mathrm{c} \tau)^{4}\right]
$$

The contribution of the efficiency modelling to the systematic uncertainty is evaluated by varying these parameters and determining the effect on the fit for $\Delta m_{d}$ in Chapter 8; studies reveal that the only significant contribution is the variation of $p_{1}$, the "rise time" of the sigmoid. Accordingly, the systematic uncertainty from $\epsilon(\mathrm{c} \tau)$ is evaluated by setting $p_{3}$ through $p_{6}$ to zero and varying only $p_{1}$. Two curves representing the limits of the variation are also shown in Figures 5.4 and 5.5.

\subsection{Sequential Lepton Fraction}

The relative fractions of different $b$ hadron decay processes producing trigger leptons determine the expected correlation of the lepton's charge with the $b$ flavor. Owing to the requirement of a displaced-vertex tag, the fractions may be different from the 


\section{Electron Trigger}
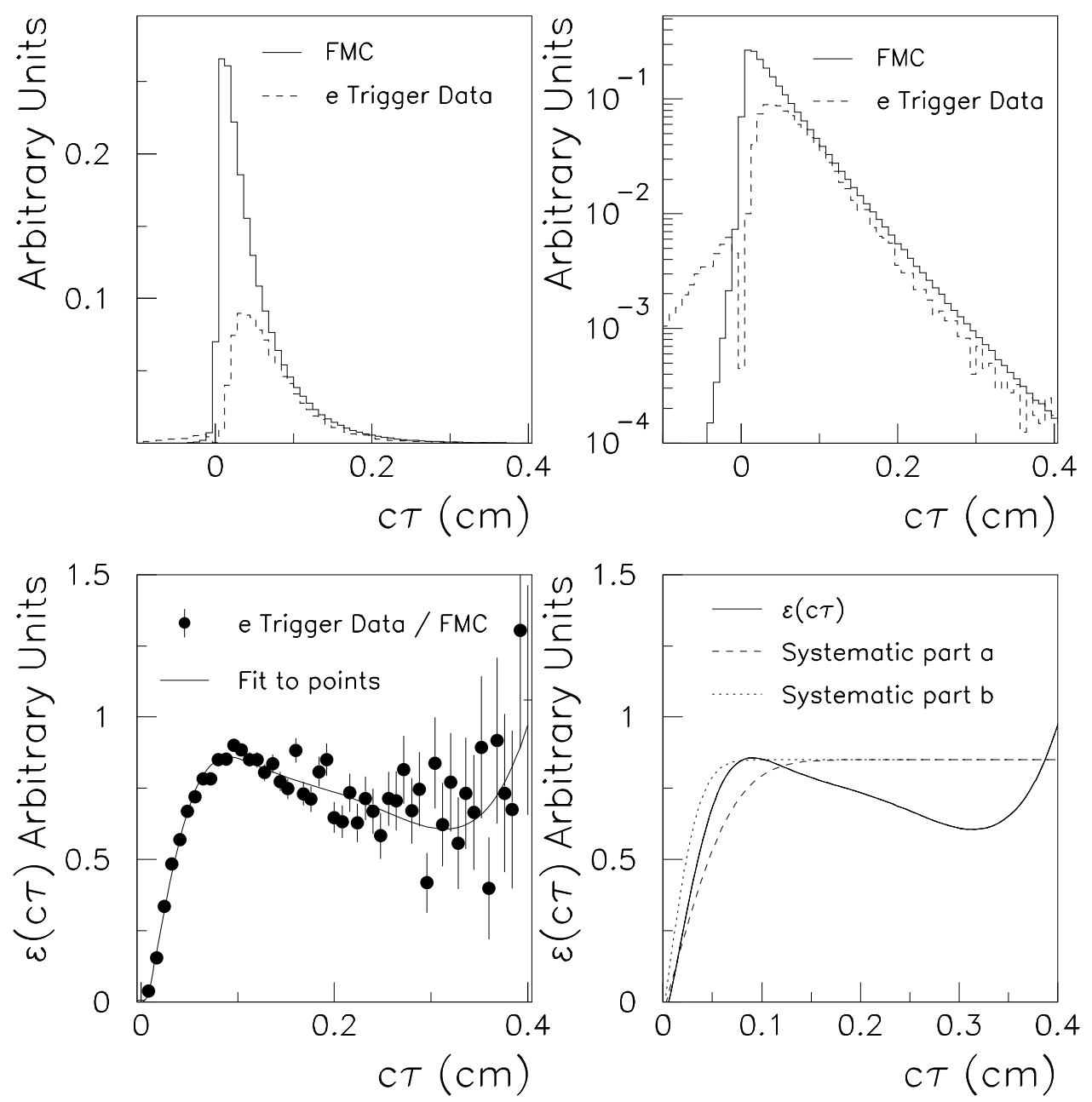

Figure 5.4: Top Left: The trigger electron data and FMC reconstructed $\mathrm{c} \tau$ distributions on a linear scale.

Top Right: The same distributions on a log scale.

Bottom Left: The trigger electron reconstructed $\mathrm{c} \tau$ distribution divided by the FMC distribution, overlaid with the fit of Equation 5.5.

Bottom Right: The fit result for $\epsilon(\mathrm{c} \tau)$ and the two curves used in the evaluation of the systematic uncertainty. 


\section{Muon Trigger}
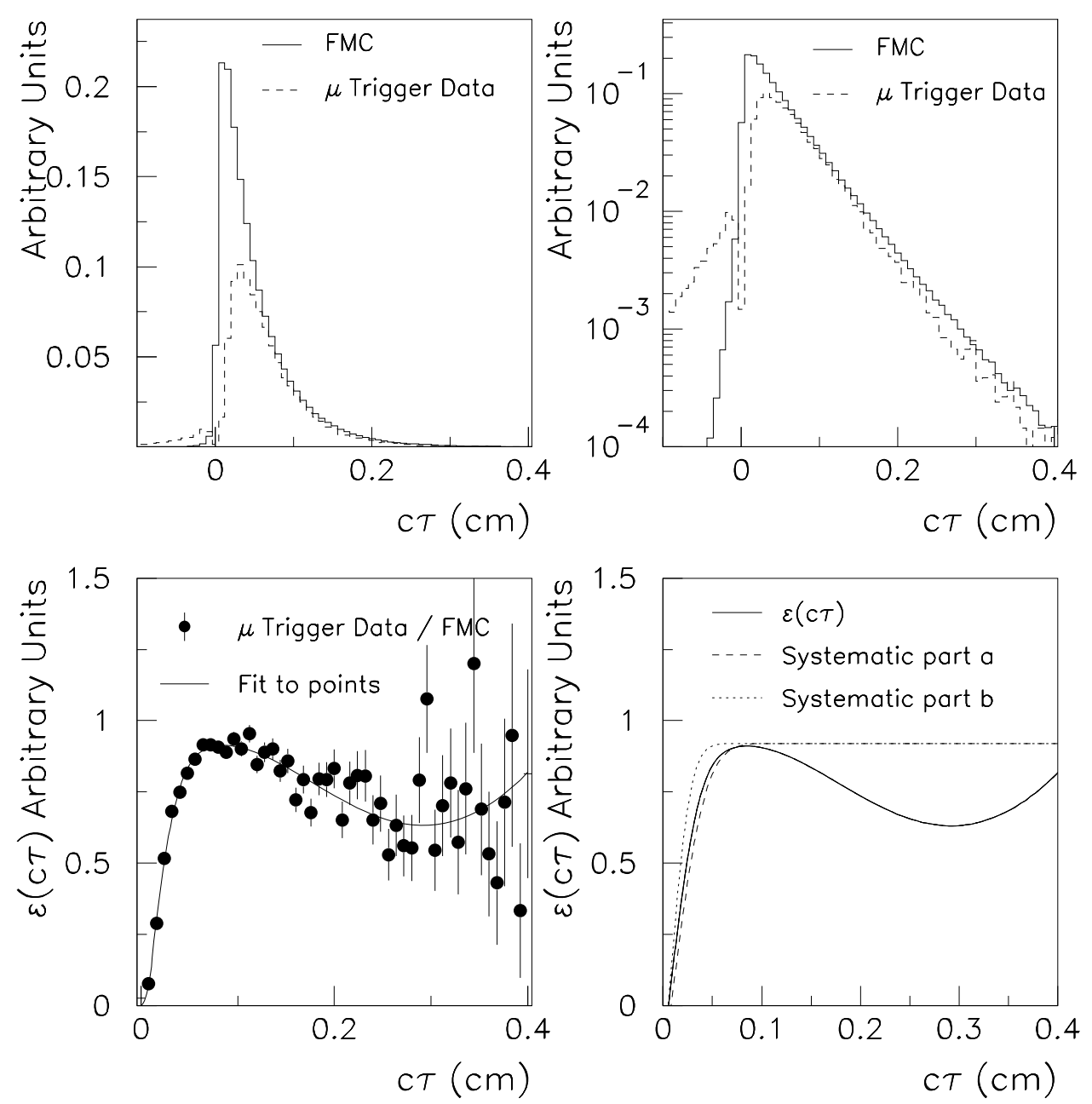

Figure 5.5: Top Left: The trigger electron data and FMC reconstructed $\mathrm{c} \tau$ distributions on a linear scale.

Top Right: The same distributions on a log scale.

Bottom Left: The trigger electron reconstructed $\mathrm{c} \tau$ distribution divided by the FMC distribution, overlaid with the fit of Equation 5.5.

Bottom Right: The fit result for $\epsilon(\mathrm{c} \tau)$ and the two curves used in the evaluation of the systematic uncertainty. 


\begin{tabular}{|c||c|c|c|c|c|c|}
\hline & $\begin{array}{c}b \rightarrow \ell \\
\text { in } \%\end{array}$ & $\begin{array}{c}b \rightarrow c \rightarrow \ell \\
\text { in } \%\end{array}$ & $\begin{array}{c}b \rightarrow \bar{c} \rightarrow \ell \\
\text { in } \%\end{array}$ & $\begin{array}{c}b \rightarrow \tau \rightarrow \ell \\
\text { in } \%\end{array}$ & $\begin{array}{c}b \rightarrow J / \psi \rightarrow \ell^{+} \ell^{-} \\
\text {in } \%\end{array}$ & $\begin{array}{c}\text { Dalitz } \\
\text { in } 10^{-4}\end{array}$ \\
\hline & $\mathrm{RS}$ & $\mathrm{WS}$ & $\mathrm{RS}$ & $\mathrm{RS}$ & $\frac{1}{2}$ & $\frac{1}{2}$ \\
\hline \hline$e:$ & $92.1 \pm 0.6$ & $3.6 \pm 0.1$ & $0.6 \pm 0.0$ & $1.1 \pm 0.1$ & $2.6 \pm 0.1$ & $3.9 \pm 0.9$ \\
\hline$\mu:$ & $80.6 \pm 0.5$ & $11.8 \pm 0.2$ & $3.6 \pm 0.1$ & $1.5 \pm 0.1$ & $2.4 \pm 0.1$ & - \\
\hline
\end{tabular}

Table 5.1: Sources of the trigger lepton in the electron and muon $b \bar{b}$ Monte Carlo samples, where the given errors are only from the Monte Carlo statistics and do not include errors on the relative branching ratios, as determined by the Particle Data Group. Also indicated is whether the lepton has the correct charge correlation with the $b$ quark (RS), the incorrect correlation (WS), or half-RS/half-WS. The decay $b \rightarrow \ell$ is direct decay, all others are sequential decays.

standard PDG branching ratios, as embodied by the QQ decay table. Consequently, they are determined directly from the SECVTX-tagged $b \bar{b}$ Monte Carlo samples. The fractions of leptons from direct $b \rightarrow \ell$ decays, sequential $b \rightarrow c \rightarrow \ell$ decays, $b \rightarrow \bar{c} \rightarrow \ell$ decays (where $b \rightarrow W \rightarrow \bar{c} s$ ), $b \rightarrow \tau \rightarrow \ell$ decays, and $b \rightarrow J / \psi \rightarrow$ $\ell^{+} \ell^{-}$decays are shown in Table 5.1. Electrons may also originate from Dalitz decays $\left(\pi^{0} \rightarrow \gamma e^{+} e^{-}\right)$, and these are included in the table as well. Collectively, the fraction of leptons from wrong-sign sequentials $\left(P_{w s}^{s e q}\right)$, those non-direct decays which produce leptons anti-correlated with the $b$ flavor, is of primary interest. The right-sign/wrong-sign nature of the various $b$ decays is also indicated in Table 5.1.

The sequential fraction is not constant with $\mathrm{c} \tau$. Intuitively, $b$ hadrons with a very short lifetime are unlikely to enter the sample over the very slow SECVTX efficiency turn-on (see Section 5.3), unless the decay distance is "enhanced" by the charm lifetime. The sequential fraction as a function of true and reconstructed $\mathrm{c} \tau$ for the electron and muon $b \bar{b}$ Monte Carlo samples is shown in Figure 5.6. The figure also shows the fraction of wrong-sign sequential decays $\left(P_{w s}^{s e q}\right)$ as a function of true and reconstructed $\mathrm{c} \tau$. Clearly, the sequential fraction is falling with true $\mathrm{c} \tau$, matching intuition. 

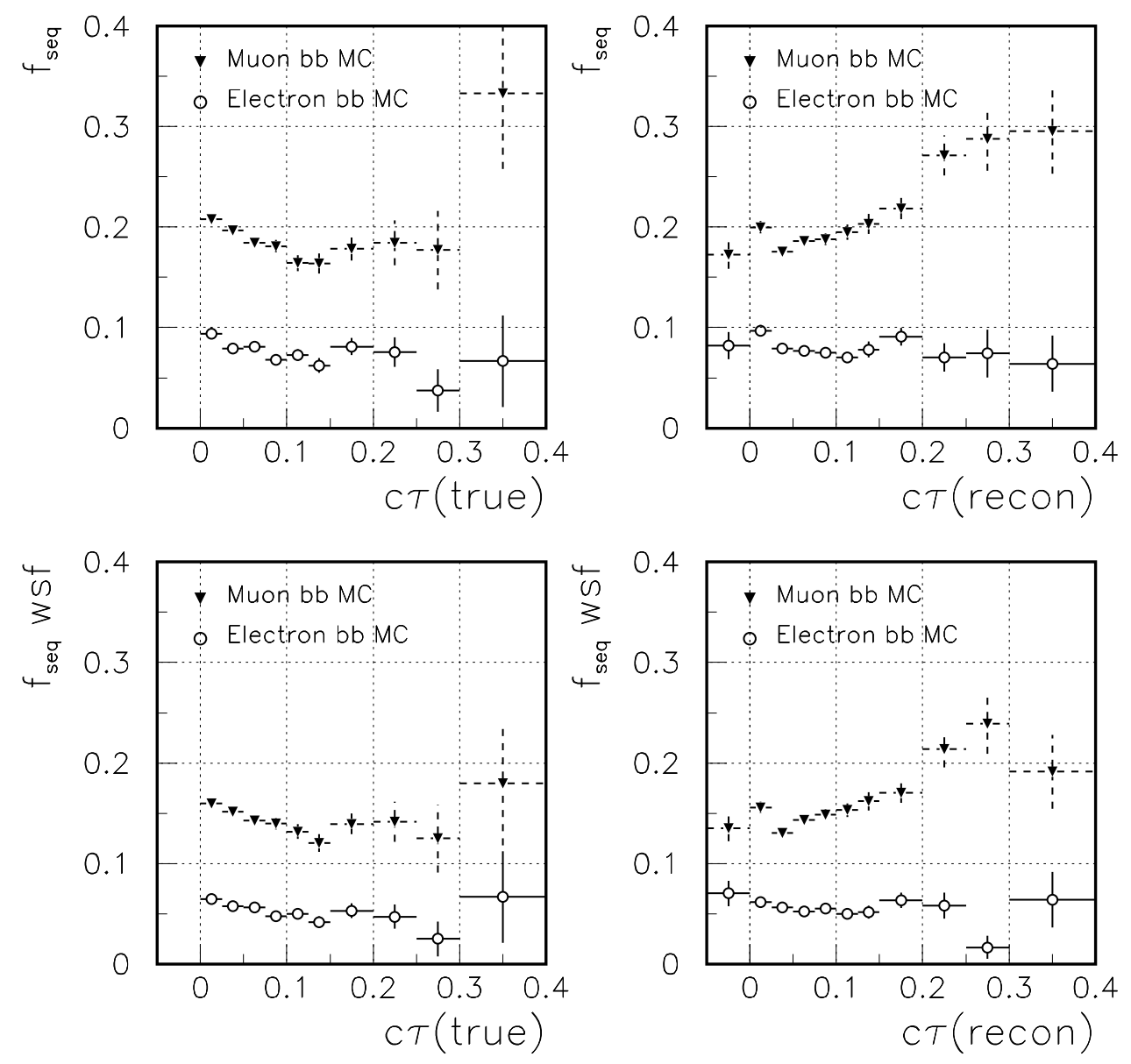

Figure 5.6: Sequential fractions versus $\mathrm{c} \tau$.

Top left: The sequential fraction as a function of the true $\mathrm{c} \tau$ in electron and muon $b \bar{b}$ Monte Carlo.

Top right: The sequential fraction versus reconstructed $\mathrm{c} \tau$.

Bottom left: The wrong-sign sequential fraction $P_{w s}^{s e q}$ (as defined in the text) versus true $\mathrm{c} \tau$.

Bottom right: $P_{w s}^{s e q}$ versus reconstructed $\mathrm{c} \tau$. 


\section{Chapter 6}

\section{Sample Composition}

The origin of the events in the data samples developed in Chapter 4 must be known,

if the $B_{d}^{0}$ mixing measurement is to proceed. The requirement that a SECVTX tag be associated with the trigger lepton preferentially selects events from $b \bar{b}$ production, owing to the large $b$ lifetime. Nevertheless, some background still remains from direct charm production $(c \bar{c} \rightarrow \ell+X)$ and fake leptons (hadrons mistakenly identified as trigger leptons). Fake leptons may come from any source; that is, they may be decay products from $b \bar{b}$ or $c \bar{c}$ production, fragmentation particles, or particles from generic light quark or gluon jets. To study the origins of the data samples' events, as well as their effect on flavor-tagging, representative samples must be assembled for each source.

This chapter documents how the relative fractions of events from $b \bar{b}, c \bar{c}$, and fake leptons are determined. The Monte Carlo samples described in the previous chapter are used to model the $b \bar{b}$ and $c \bar{c}$ events in the data samples. The creation of samples of fake leptons from the ECLB and MULB BPAD datasets, to model the fake leptons in the data samples, is described here.

The method used to determine the sample composition is to take a kinematic 
variable which has markedly different distribution for $b \bar{b}, c \bar{c}$, and fake events, create templates from the distributions of the three samples, and fit the templates to the data distributions. The fit then yields the relative fractions of each in the data. Two such kinematic variables are used; the transverse momentum of the trigger lepton relative to the other tracks in the $b$ jet $\left(p_{\mathrm{t}}^{\mathrm{rel}}\right)$, and the invariant mass of the tracks in the SECVTX tag $\left(M_{c l}\right)$. The fit to $M_{c l}$ is employed as a cross-check to the $p_{\mathrm{t}}^{\mathrm{rel}}$ fit, providing an estimate of the systematic uncertainty associated with the determination of the sample composition.

\subsection{Fake Lepton Samples}

The fake lepton selection criteria embody a reversal of the trigger lepton criteria, as the goal is to assemble a dataset containing non-leptons. The fake electron sample was selected from the ECLB BPAD dataset by inverting the HAD/EM cut $(\mathrm{HAD} / \mathrm{EM}>0.04$ instead of $<0.04$, keeping all other cuts the same) and requiring that the $d E / d x$ of the fake electron track be inconsistent with the electron hypothesis by 3 standard deviations, resulting in a sample of 10,731 events, of which only 234 have SECVTX tags in the fake electron jet.

Fake muons are selected in two ways, both of which are selected for the signatures of hadrons which penetrate the calorimeter and muon shielding to leave hits in the various muon detectors (so-called punchthrough muons). First, hadrons which penetrate to the CMU muon detectors can saturate the CMU ADC's if they have begun to shower; this disallows a valid $z$ measurement, and production marks these stubs with a negative $\chi_{z}^{2}(\mathrm{CMU})$. There are 12,932 events with a muon which meets all other trigger muon cuts, but has a negative $\chi_{z}^{2}(\mathrm{CMU})$. Second, muons can be selected which meet all trigger muon CMU requirements and whose tracks project 
into the fiducial volume of the CMP chambers but fail to be matched to a CMP stub. These are likely to be hadrons which have penetrated to the CMU but had an hadronic interaction in the CMP shielding; fiducial real muons of $6 \mathrm{GeV} / \mathrm{c}$ and above rarely fail to leave a stub in the CMP. Unfortunately, there is no Stream B trigger which corresponds to such CMU-only muons, so the inclusive lepton datasets are searched for "volunteer" fake muons. 10,670 events from the ECLB and MULB datasets are found in this manner. Of the 23,602 total fake muon events, 1,582 have a SECVTX tag in the fake muon jet.

Figures 6.1 and 6.2 compare the reconstructed $\mathrm{c} \tau$ distributions ${ }^{1}$ of the fake lepton samples to the data samples. The agreement is quite good, indicating that the fake leptons most likely have an origin from $b \bar{b}$ decay. The comparison is made to the data samples, as the fake lepton samples were also obtained from the data; for the sake of completeness, however, Figure 6.3 plots the $L_{x y}$ distributions of the fake and $b \bar{b}$ Monte Carlo samples together. The agreement is excellent. Accordingly, this analysis will proceed under the assumption that in the case of the fake lepton samples, SECVTX has selected $b \bar{b}$ events where a daughter hadron from $b$ hadron decay was misidentified as a lepton.

\footnotetext{
${ }^{1}$ Where $c \tau$ is calculated according to the prescription of Section 5.2.
} 


\section{Fake Electrons}
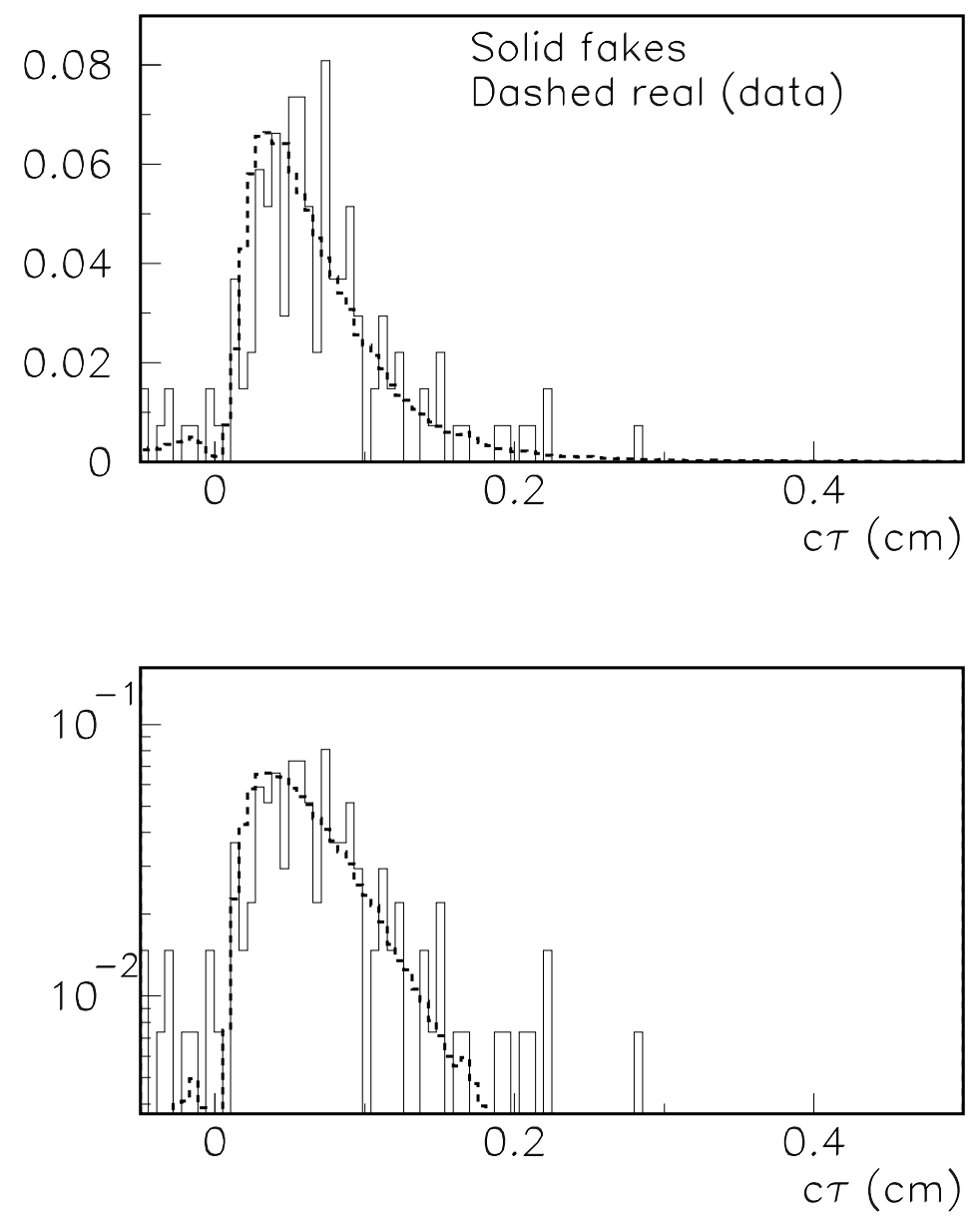

Figure 6.1: Reconstructed $\mathrm{c} \tau$ distributions for the fake electron (solid) and trigger electron data (dashed) samples, on linear (top) and logarithmic (bottom) scales. The distributions are normalized to unit area. 


\section{Fake Muons}
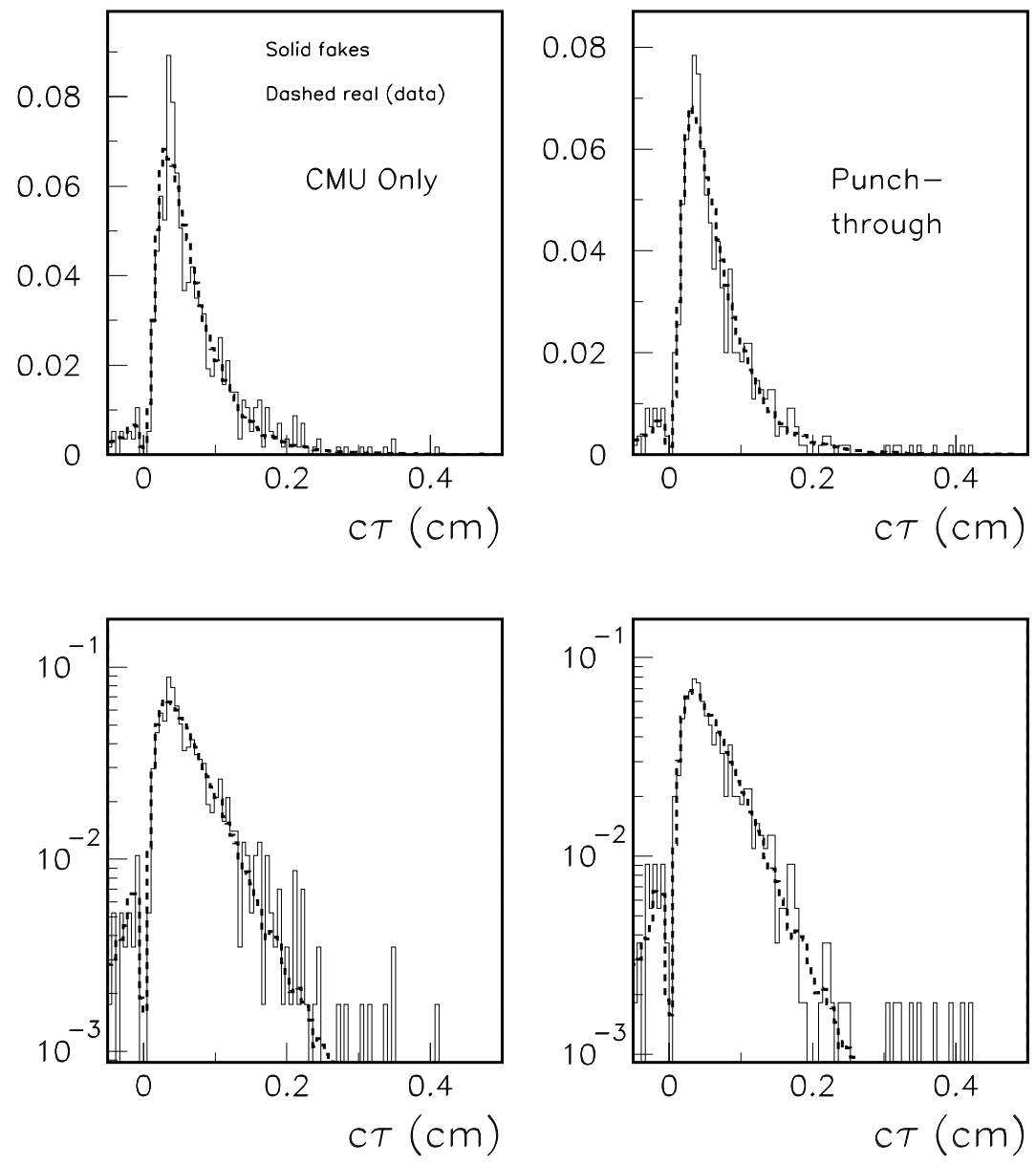

Figure 6.2: Reconstructed $\mathrm{c} \tau$ distributions for the fake muon (solid) and trigger muon data (dashed) samples, on linear (top) and logarithmic (bottom) scales. The distributions are normalized to unit area. 

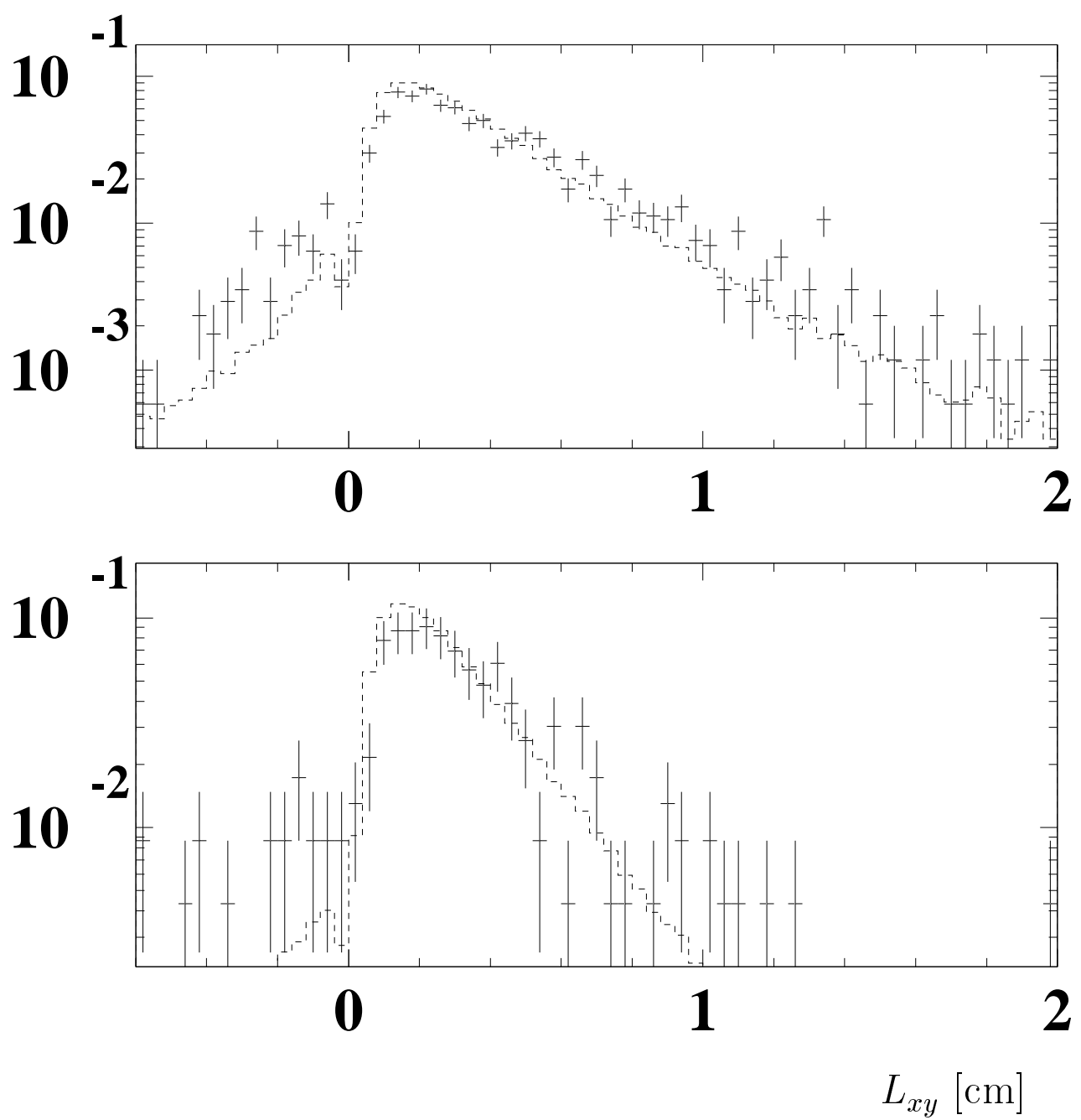

Figure 6.3: Comparison of the $L_{x y}$ distribution of SECVTX-tagged fake events (crosses) to $b \bar{b}$ Monte Carlo (dashed) for trigger muons (top) and electrons (bottom). The distributions are normalized to unit area. 


\section{$6.2 \quad p_{\mathrm{t}}^{\mathrm{rel}}$ Method}

Traditionally, $p_{\mathrm{t}}^{\text {rel }}$ is defined as the transverse momentum of the lepton track relative to the $b$ jet. For a direct $b \rightarrow \ell$ decay, the momentum transfer can be as large as half the $b$ quark mass (in the limit $M_{\ell} \ll M_{b}$ ). This analysis makes use of an alternative definition of $p_{\mathrm{t}}^{\text {rel }}$, in which the lepton's momentum is first subtracted from the jet's; in this case the momentum transfer can be as large as the $b$ quark mass. This definition is used because Monte Carlo studies indicate that the separation between leptons from direct $b$ decay and from direct charm decay is larger with this choice.

The $p_{\mathrm{t}}^{\text {rel }}$ of a track is determined by first selecting the jet in which it is located, and then calculating

$$
\begin{aligned}
\overrightarrow{\mathrm{p}}_{j e t}^{\prime} & =\overrightarrow{\mathrm{p}}_{\text {jet }}-\overrightarrow{\mathrm{p}}_{\text {track }} \\
\cos \theta & =\frac{\overrightarrow{\mathrm{p}}_{\text {track }} \cdot \overrightarrow{\mathrm{p}}_{\text {jet }}^{\prime}}{\left|\overrightarrow{\mathrm{p}}_{\text {track }}\right|\left|\overrightarrow{\mathrm{p}}_{\text {jet }}^{\prime}\right|} \\
p_{\mathrm{t}}^{\mathrm{rel}} & =\left|\overrightarrow{\mathrm{p}}_{\text {track }}\right| \sin \theta
\end{aligned}
$$

For the calculation of $p_{\mathrm{t}}^{\mathrm{rel}}$, the direction of the $b$ jet is found using the TRKSJT CTC track-based jet clustering algorithm, described in Section 4.5, as opposed to the standard calorimeter-based algorithm. The $\eta-\phi$ cone used for the clustering is $\Delta R=0.4$, rather than $\Delta R=0.8$, for reasons which will be discussed below.

\subsubsection{Distributions and Fits}

The number of tracks in trigger electron and muon jets $\left(N_{\text {trk }}^{\text {jet }}\right)$ is shown in Figure 6.4. It is clear that some leptons are the only tracks in their jet, rendering a determination of $p_{\mathrm{t}}^{\text {rel }}$ impossible. The mean number of tracks in electron jets is also lower than 

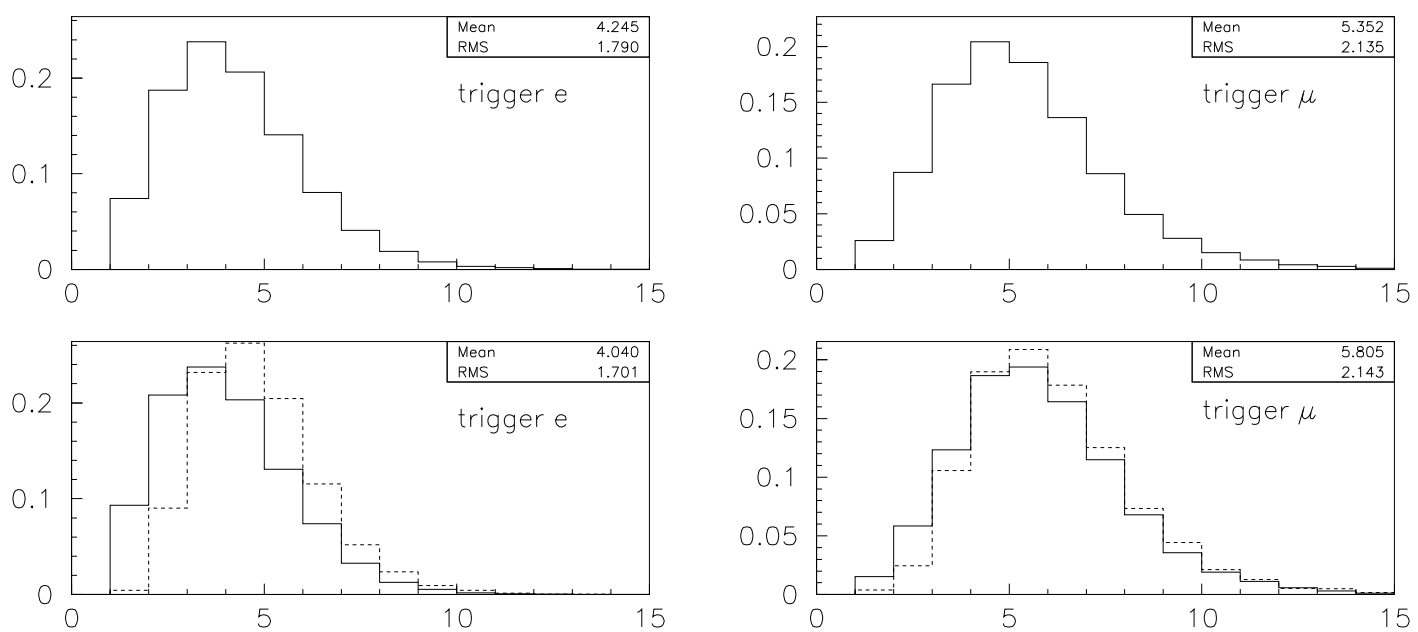

Figure 6.4: Upper left: the number of tracks in the trigger jet for trigger electron data. Lower left: the same distributions for the $b \bar{b}$ (solid) and $c \bar{c}$ (dashed) electron samples. Upper right: the number of tracks in the trigger jet for trigger muon data.

Lower right: the same distributions for the $b \bar{b}$ (solid) and $c \bar{c}$ (dashed) muon samples.

that of muon jets owing to the SECVTX minimum jet $\mathrm{E}_{\mathrm{t}}$ requirement. The central values of the sample compositions are derived from fits to the $p_{\mathrm{t}}^{\mathrm{rel}}$ distributions, using only jets with $N_{\text {trk }}^{\text {jet }} \geq 5$ for reasons discussed below. Any bias to the sample composition from the exclusion of events with $N_{\text {trk }}^{\text {jet }} \leq 4$ shall be investigated, both through varying this requirement and using a fit to the SECVTX cluster mass distribution as a cross-check.

Figures 6.5 and 6.6 show the trigger lepton $p_{\mathrm{t}}^{\mathrm{rel}}$ distribution for the data, $b \bar{b}$ and $c \bar{c}$ Monte Carlo, and fake lepton samples, where each has been normalized to unit area. The shapes of the last three are parametrized by fitting the function

$$
f(x)=P_{1} \cdot x^{P_{2}} \cdot \exp \left(-P_{3} \cdot x^{P_{4}}\right)+\frac{P_{5}}{P_{7}} \cdot \exp \left(-\frac{1}{2}\left[\frac{x-P_{6}}{P_{7}}\right]^{2}\right)
$$

to the distributions, producing templates for the sample composition fit. The derived parameters $P_{1}-P_{7}$ are given in the insets in Figures 6.5 and 6.6, and the 
templates overlaid on the plots. The parametrizations are excellent.

The fake samples present a challenge: the SECVTX-tagged samples are quite small (234 electron events and 1,582 muon events). The low statistics introduce a larger uncertainty into the templates; therefore, the fake $p_{\mathrm{t}}^{\mathrm{rel}}$ distributions shown in Figures 6.5 and 6.6 (and the associated parametrizations forming the templates) are for the entire fake samples, omitting the SECVTX tag requirement. Possible bias resulting from this estimate of the fakes' $p_{\mathrm{t}}^{\text {rel }}$ spectrum is investigated in Section 6.2.1.

The templates are used in a three-component fit to the trigger electron and muon $p_{\mathrm{t}}^{\text {rel }}$ distributions, yielding $f_{b \bar{b}}, f_{\bar{c} \bar{c}}$, and $f_{f a k e}$. The fit results are displayed graphically in Figure 6.7 and numerically in Table 6.1. The smaller charm fraction in the electron sample is due to the implicit isolation requirements of the electron trigger.

$N_{\text {trk }}^{\text {jet }}$ Requirement and $\Delta R$ Cone Size

The $N_{\mathrm{trk}}^{\mathrm{jet}} \geq 5$ requirement, which retains only $29.5 \%$ of trigger electron events and $51.7 \%$ of trigger muon events, was imposed because including jets with a smaller number of tracks leads to a lower-quality fit, as shown below. Again, the large differences between the two samples is due to the SECVTX minimum jet $\mathrm{E}_{\mathrm{t}}$ requirement. To demonstrate that there is little bias in determining the sample composition from this subsample, the template derivation and fitting procedure is repeated anew for the sequence of minimum $N_{\text {trk }}^{\text {jet }}$ from 2 to 7 , using trigger electron data. The resulting fractions and $\chi^{2}$ per degree of freedom are shown in Table 6.2.

The numbers clearly show that there is no strong bias for the range of cuts,

except possibly for the $N_{\text {trk }}^{\text {jet }} \geq 2$ and $N_{\text {trk }}^{\text {jet }} \geq 3$ fits, in which the $c \bar{c}$ and fake templates become substantially more similar, hindering the fit from distinguishing between 

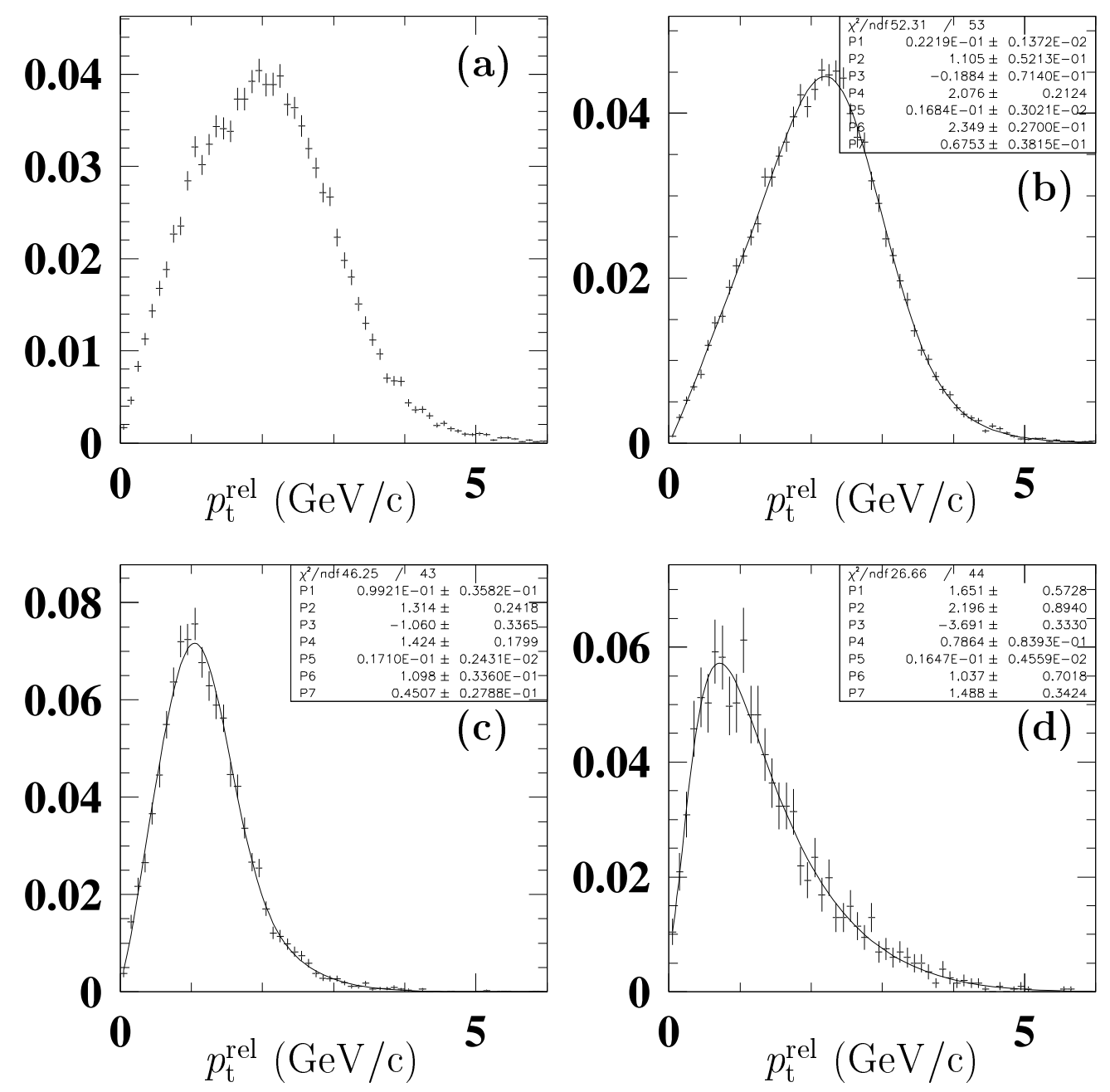

Figure 6.5: Trigger electron $p_{\mathrm{t}}^{\mathrm{rel}}$ distributions for (a) data, (b) $b \bar{b}$ Monte Carlo, (c) $c \bar{c}$ Monte Carlo, and (d) fakes. The functional form of the template parametrization is given in the text. 

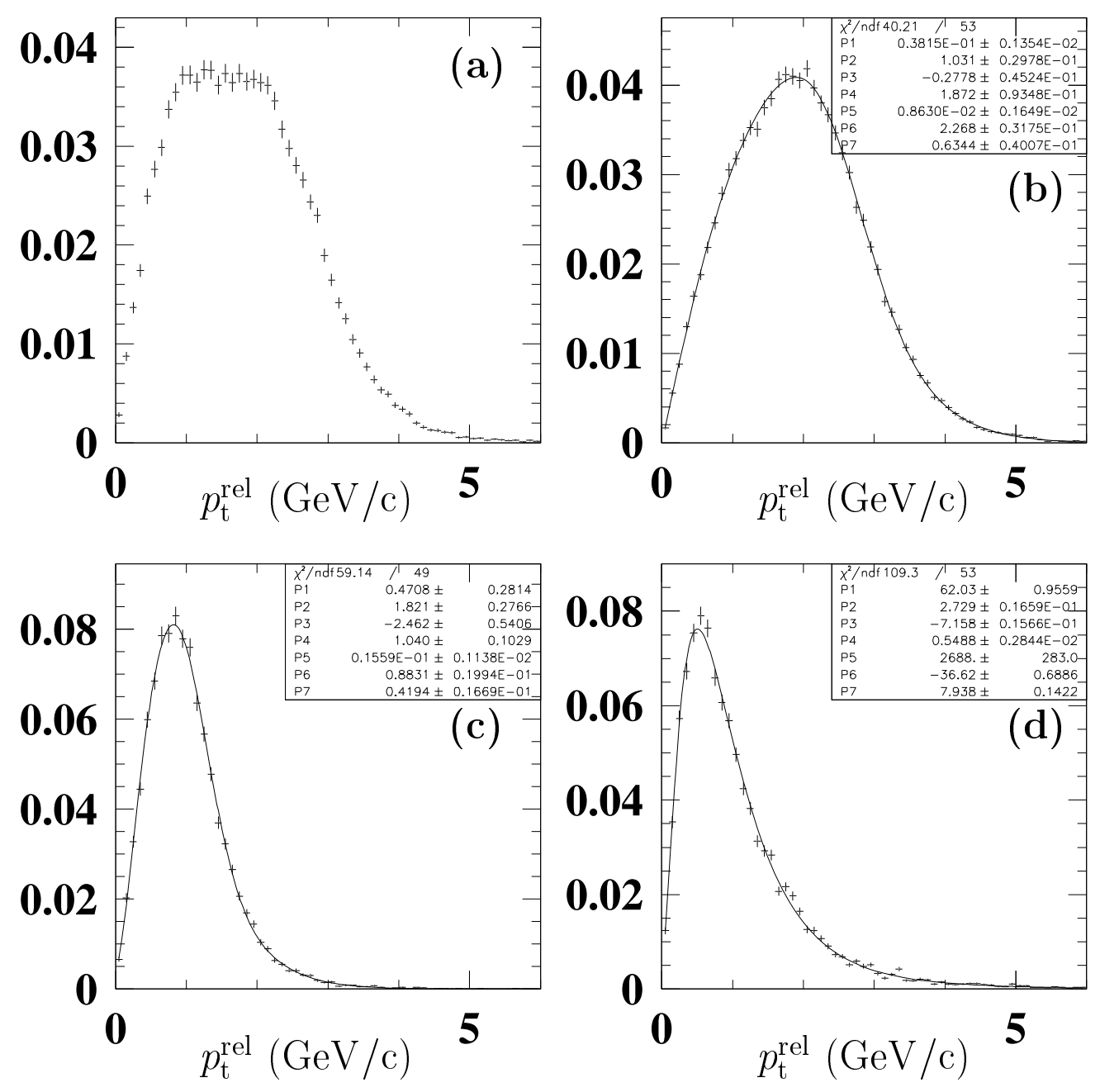

Figure 6.6: Trigger muon $p_{\mathrm{t}}^{\mathrm{rel}}$ distributions for (a) data, (b) $b \bar{b}$ Monte Carlo, (c) $c \bar{c}$ Monte Carlo, and (d) fakes. The functional form of the template parametrization is given in the text. 


\begin{tabular}{|c||c|c|c|}
\hline & $f_{b \bar{b}}$ & $f_{c \bar{c}}$ & $f_{\text {fake }}$ \\
\hline \hline$e:$ & $(85.2 \pm 0.8) \%$ & $(8.1 \pm 1.4) \%$ & $(6.7 \pm 1.5) \%$ \\
\hline$\mu:$ & $(84.3 \pm 0.6) \%$ & $(11.6 \pm 1.1) \%$ & $(4.1 \pm 1.1) \%$ \\
\hline
\end{tabular}

Table 6.1: The $b \bar{b}, c \bar{c}$, and fake fractions determined from the three-component fit of the templates to the data.
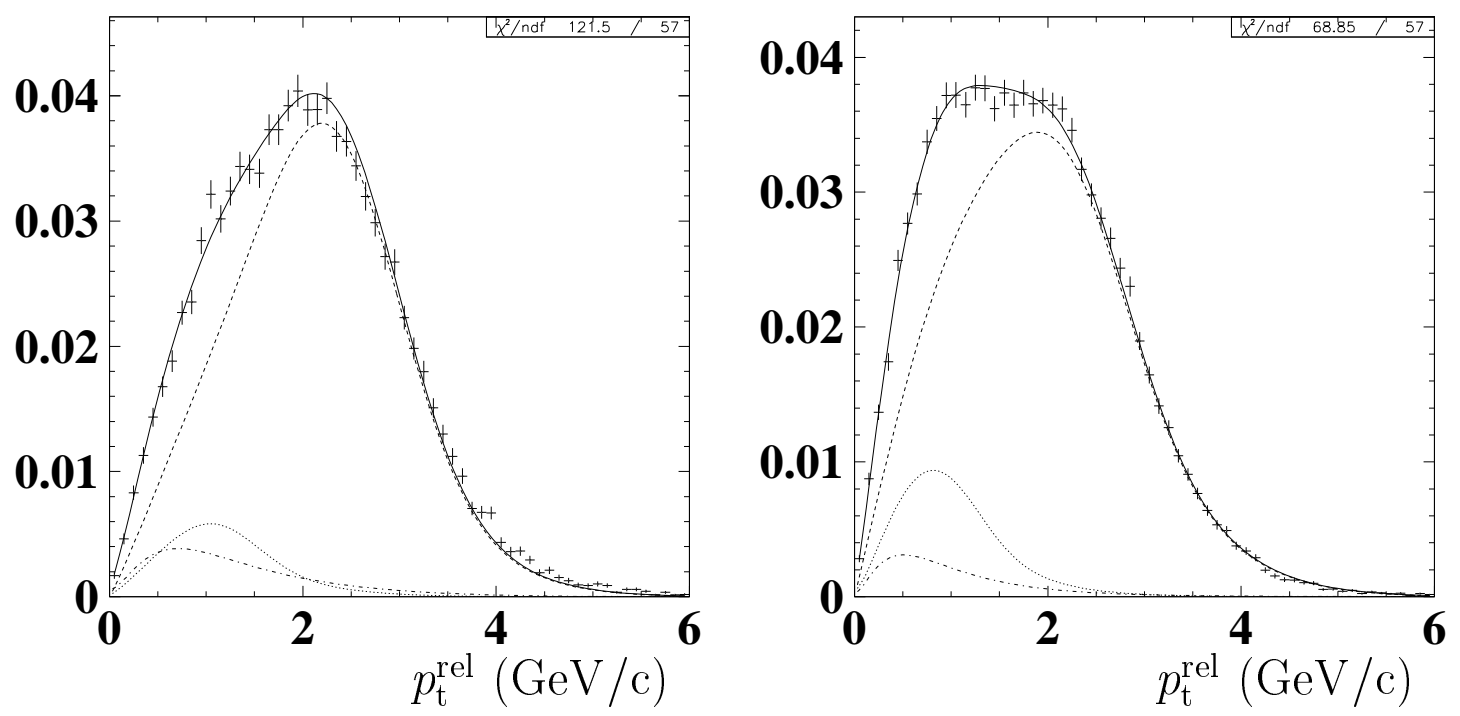

Figure 6.7: Results of the template fits (solid) to the trigger electron (left) and muon (right) data (crosses). The individual contributions from the $b \bar{b}$ (dashed), $c \bar{c}$ (dotted), and fake (dashed-dotted) templates are also shown. 
them. At the highest $N_{\text {trk }}^{\text {jet }}$ requirements, there is perhaps a hint of a bias, as $b$ jets, on average, have a lower track multiplicity than charm jets. Thus the $N_{\text {trk }}^{\text {jet }} \geq 7$ requirement may actually result in a smaller $f_{b \bar{b}}$, though with the available statistics it appears to be only a $2 \sigma$ effect. Table 6.2 also indicates why the $N_{\text {trk }}^{\text {jet }} \geq 5$ requirement was chosen: it is the fit with the lowest $N_{\text {trk }}^{\text {jet }}$ cut with a $\chi^{2}$ per degree of freedom considered acceptable. Figure 6.8 shows graphically how the three-component fit to jets with $N_{\text {trk }}^{\text {jet }} \geq 2$ leads to an inadequate $\chi^{2}$.

The fit to the composition of the electron sample is more sensitive to the minimum $N_{\text {trk }}^{\text {jet }}$ than that of the muon sample, owing to the smaller average track multiplicity of electron jets compared to muon jets. As the requirements on the $p_{\mathrm{t}}^{\mathrm{rel}}$ fits do not noticeably bias the determination of the trigger electron sample composition, the conclusion is that the determination of the trigger muon sample composition is also unbiased.

A similar study examining how the goodness-of-fit depends on the trackclustering cone size reveals that a cone of $\Delta R=0.4$ is the optimal size for obtaining a reasonable $\chi^{2}$. Again, the composition fractions do not vary noticeably over a range of choices for $\Delta R$; the smaller cone size just ensures confidence in the fit. Figure 6.8 shows the less-than-perfect three-component fit from a cone size of $\Delta R=0.8$. 


\begin{tabular}{|l||c|c|c|c|}
\hline & $f_{b \bar{b}}$ & $f_{c \bar{c}}$ & $f_{\text {fake }}$ & $\chi^{2} /$ dof \\
\hline \hline$N_{\text {tr }}^{\text {jet }} \geq 2$ & $(85.0 \pm 0.6) \%$ & $(2.1 \pm 0.6) \%$ & $(12.9 \pm 0.9) \%$ & 5.1 \\
$N_{\text {trk }}^{\text {jet }} \geq 3$ & $(86.3 \pm 0.6) \%$ & $(4.8 \pm 0.9) \%$ & $(8.8 \pm 1.1) \%$ & 4.3 \\
$N_{\text {tre }}^{\text {jet }} \geq 4$ & $(85.8 \pm 0.6) \%$ & $(6.8 \pm 1.0) \%$ & $(7.4 \pm 1.2) \%$ & 2.6 \\
$N_{\text {trk }}^{\text {jet }} \geq 5$ & $(85.0 \pm 0.8) \%$ & $(8.1 \pm 1.4) \%$ & $(6.7 \pm 1.5) \%$ & 2.1 \\
$N_{\text {tr }}^{\text {jet }} \geq 6$ & $(83.4 \pm 1.1) \%$ & $(8.6 \pm 2.0) \%$ & $(7.7 \pm 2.0) \%$ & 2.0 \\
$N_{\text {trk }}^{\text {jet }} \geq 7$ & $(82.6 \pm 1.5) \%$ & $(8.7 \pm 2.6) \%$ & $(8.3 \pm 2.6) \%$ & 1.4 \\
\hline
\end{tabular}

Table 6.2: Results of $p_{\mathrm{t}}^{\text {rel }}$ fits for $f_{b \bar{b}}, f_{c \bar{c}}$, and $f_{f a k e}$ in the Run $1 \mathrm{~B}$ electron data for different minimum $N_{\text {trk }}^{\text {jet }}$ requirements. The fit $\chi^{2} / d o f$ is also shown.
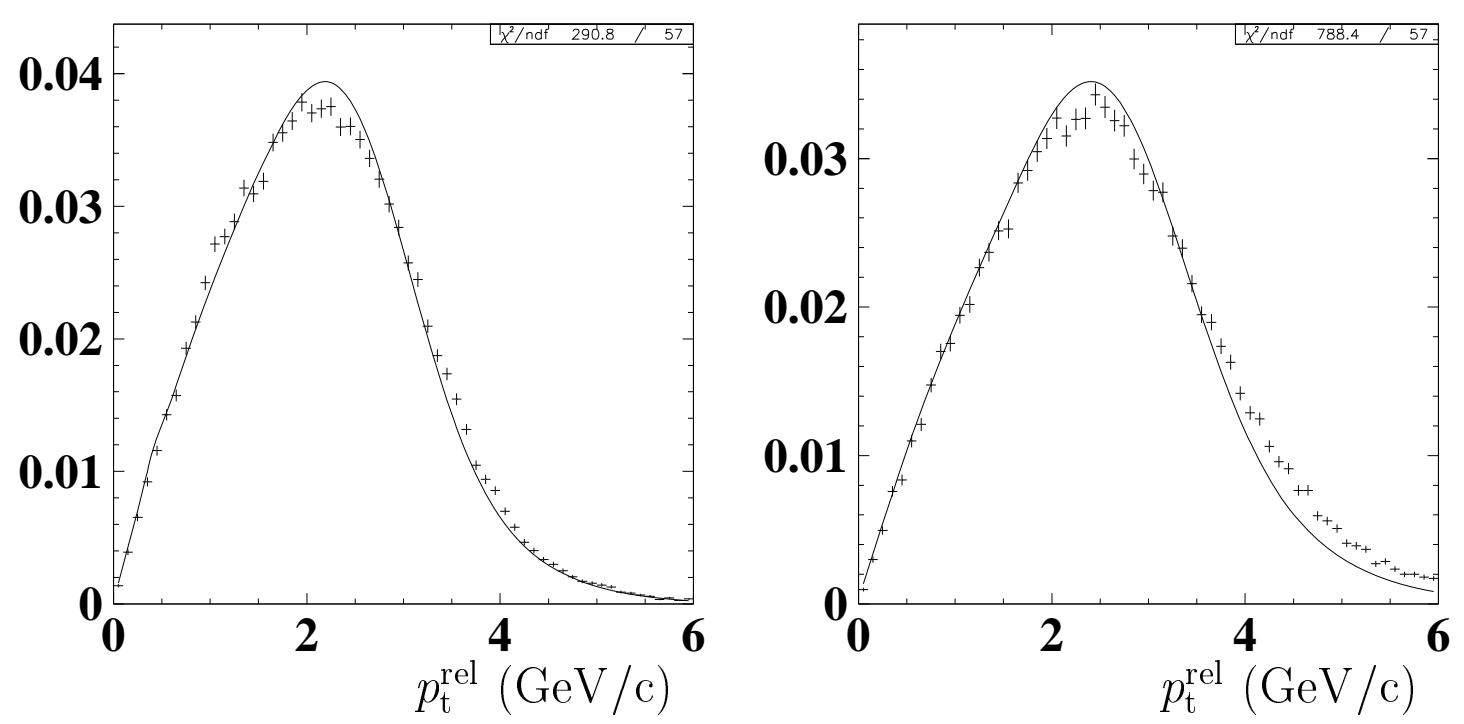

Figure 6.8: Results of the fit of $b \bar{b}, c \bar{c}$, and fake templates to the $p_{\mathrm{t}}^{\text {rel }}$ distribution of trigger electron data requiring $N_{\text {trk }}^{\text {jet }} \geq 2$ (left) and a jet clustering cone of $\Delta R=0.8$ (right). 

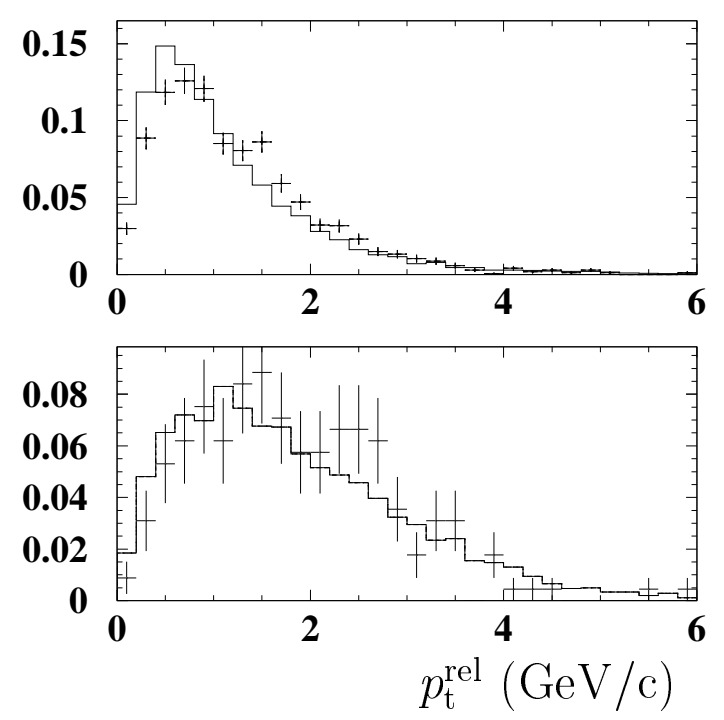

Figure 6.9: Comparison of lepton $p_{\mathrm{t}}^{\mathrm{rel}}$, requiring a SECVTX tag (crosses) and with no such requirement (solid), for fake muon (top) and fake electron (bottom) samples.

\section{$p_{\mathrm{t}}^{\text {rel }}$ Fit with the SECVTX-tagged Fake Samples}

The $p_{\mathrm{t}}^{\mathrm{rel}}$ spectrum for fake leptons in the previous fits was taken from the entire fake samples, before any SECVTX-tagging requirement. As the presence of a SECVTX tag may place a bias on $p_{\mathrm{t}}^{\text {rel }}$, any discrepancy must be investigated. Figure 6.9 shows a comparison of the $p_{\mathrm{t}}^{\text {rel }}$ spectrum of the whole fake samples to the SECVTX-tagged subsamples. Within available statistics, the fake electron subsample has the same $p_{\mathrm{t}}^{\text {rel }}$ distribution as its parent, but there is a suggestion that the fake muons associated with a tag have a harder distribution than those without. Therefore, the muon sample composition fit is repeated, using a fake template derived from the tagged subsample. The fit returns values of $f_{b \bar{b}}=(83.5 \pm 0.7) \%$, $f_{\bar{c} \bar{c}}=(11.3 \pm 1.4) \%$, and $f_{\text {fake }}=(5.2 \pm 1.6) \%$, which is consistent with the original results in Table 6.1. There is no discernable bias from using the untagged fake samples as templates. 


\subsection{Invariant Mass Method}

To check the results of the $p_{\mathrm{t}}^{\mathrm{rel}}$ fit, a second fit to the distribution of the invariant mass of the tracks in the SECVTX tag $\left(M_{c l}\right)$ is performed, assuming each track in the tag has the pion mass. The $M_{c l}$ distributions for $b \bar{b}, c \bar{c}$, fakes, and data samples are shown in Figures 6.10 and Figures 6.11 for trigger electrons and muons, respectively. For the three-component $M_{c l}$ fits to the data, no templates are created; rather, the histograms shown in the figures are fit directly to the data distribution. The fit results are given numerically in Table 6.3, and graphically in Figures 6.10 and Figures 6.11. The $p_{\mathrm{t}}^{\mathrm{rel}}$ and $M_{c l}$ fit results differ by approximately $3 \%$; the difference is assigned as a systematic uncertainty on the sample composition.

\begin{tabular}{|c||c|r|c|}
\hline & $f_{b \bar{b}}$ & \multicolumn{1}{c|}{$f_{c \bar{c}}$} & $f_{\text {fake }}$ \\
\hline \hline$e:$ & $(88.6 \pm 0.6) \%$ & $(8.1 \pm 0.4) \%$ & $(3.2 \pm 0.7) \%$ \\
$\mu:$ & $(81.1 \pm 1.2) \%$ & $(14.3 \pm 0.8) \%$ & $(4.4 \pm 1.5) \%$ \\
\hline
\end{tabular}

Table 6.3: Results of the $M_{c l}$ fit for the $b \bar{b}, c \bar{c}$, and fake fractions in the SECVTX-tagged data samples. 

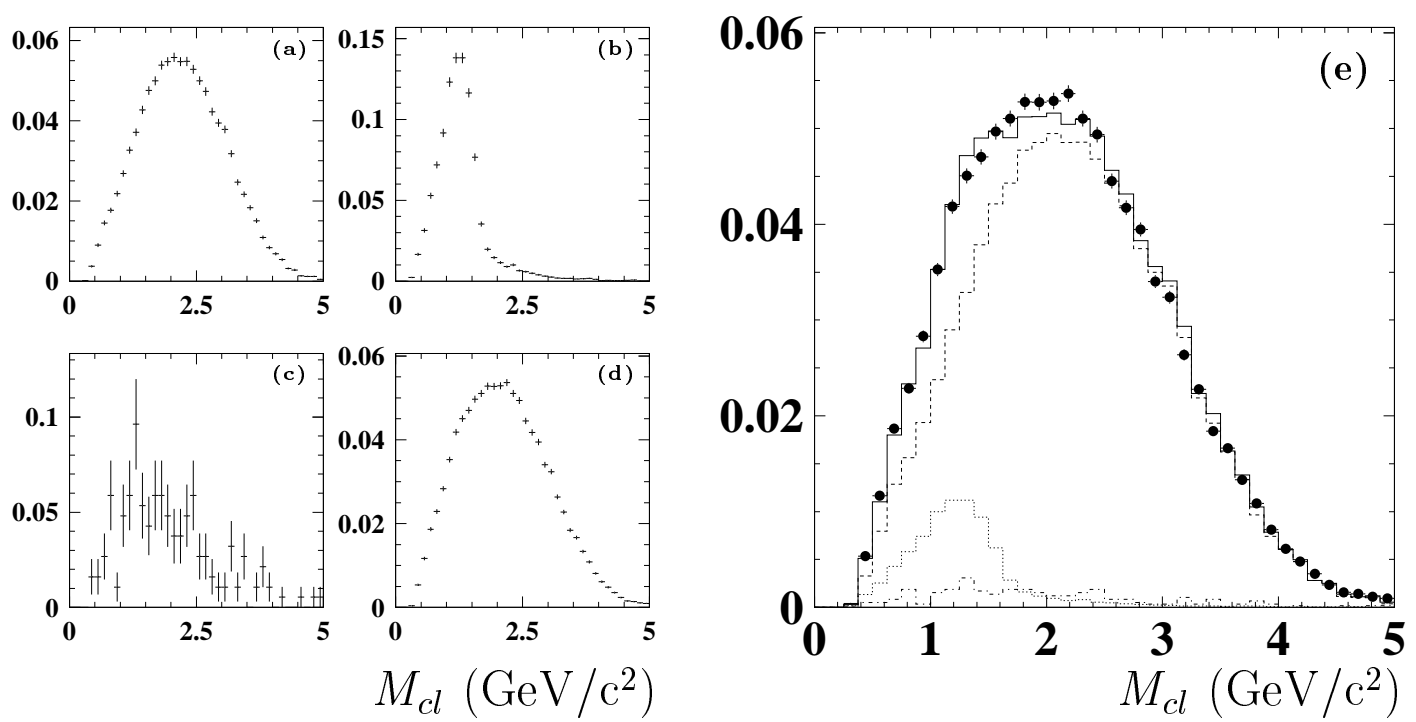

Figure 6.10: Invariant mass distribution of the SECVTX tag tracks for (a) $b \bar{b}$ Monte Carlo, (b) $c \bar{c}$ Monte Carlo, (c) fakes, and (d) data for trigger electrons. In (e) the result of fitting the $b \bar{b}, c \bar{c}$, and fake distributions to the data is displayed, where the contributions from $b \bar{b}$ (dashed), $c \bar{c}$ (dotted), and fakes (dashed-dotted) are overlaid.
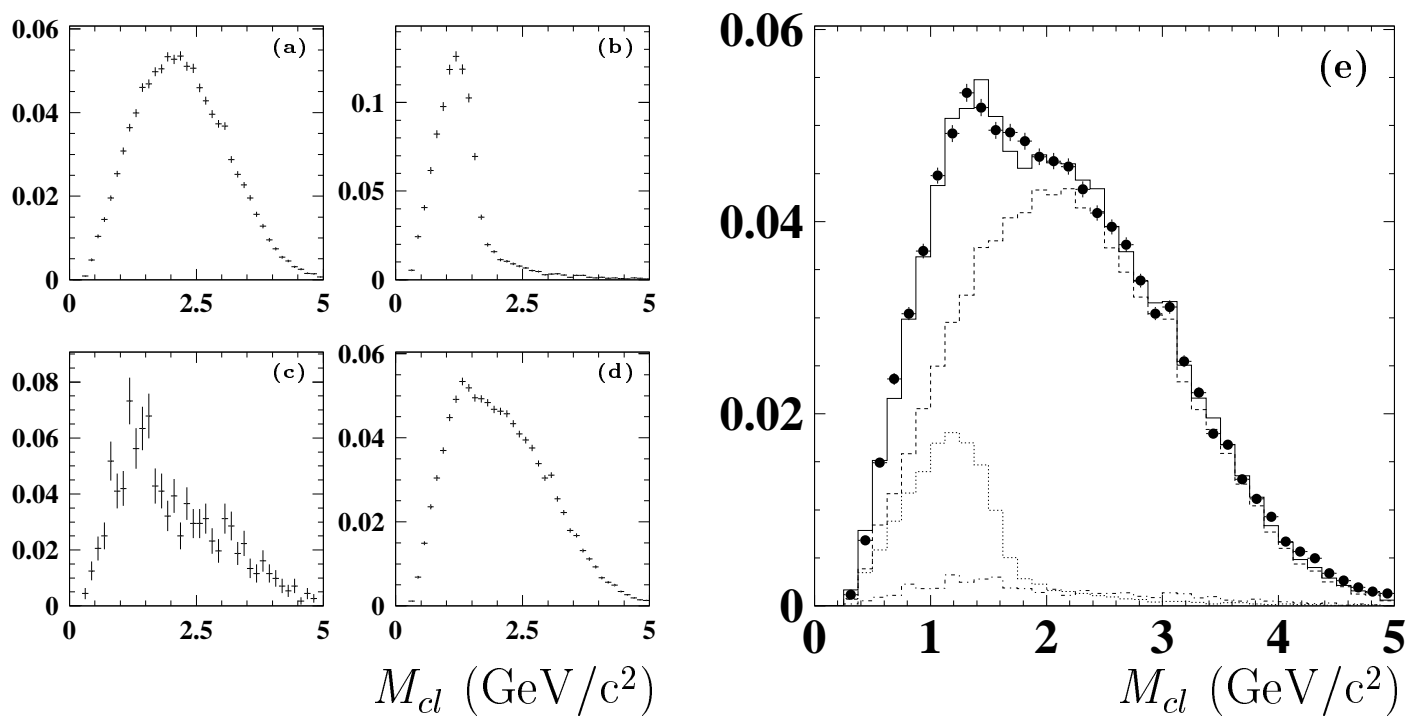

Figure 6.11: Invariant mass distribution of the SECVTX tag tracks for (a) $b \bar{b}$ Monte Carlo, (b) $c \bar{c}$ Monte Carlo, (c) fakes, and (d) data for trigger muons. In (e) the result of fitting the $b \bar{b}, c \bar{c}$, and fake distributions to the data is displayed, where the contributions from $b \bar{b}$ (dashed), $c \bar{c}$ (dotted), and fakes (dashed-dotted) are overlaid. 


\section{Chapter 7}

\section{$b$ Flavor-Tagging}

It has been mentioned many times that the charge of the trigger lepton identifies the trigger $b$ flavor at the time of its decay. In order to determine whether the trigger $b$ hadron mixed, its flavor at its production must also be identified. This analysis does not measure the production flavor directly. Rather, it relies on the fact that $b$ quarks are produced in quark-antiquark pairs by the Tevatron; the flavor of the second $b$ at its decay is thus anticorrelated with the trigger $b$ flavor, though the correlation is reduced by mixing and backgrounds. Two methods are used to locate and tag the second $b$ : low- $p_{\mathrm{t}}$ soft leptons (SLTs) from semileptonic decay and the jet charge, a momentum-weighted charge average of the tracks in the $b$ jet.

The studies in this chapter are directed towards calculating the event-by-event correct flavor-tagging probability $P_{t a g}$, which is supplied to the fit for $\Delta m_{d}$ in the next chapter. Sources of systematic biases are also investigated, but first, a discussion of flavor-tagging terminology is in order. 


\subsection{Flavor-tagging Dilution and Effective Tagging Efficiency $\left(\epsilon D^{2}\right)$}

Any flavor-tagging method can be characterized by two numbers: the efficiency $(\epsilon)$, the measure of how often the method gives any answer at all, and the dilution $(D)$, the measure of how often the method gives the right answer.

Given $N$ events, of which $N_{R}$ are tagged correctly and $N_{W}$ are tagged incorrectly, the efficiency is

$$
\epsilon=\frac{N_{R}+N_{W}}{N}
$$

and the dilution is

$$
D=\frac{N_{R}-N_{W}}{N_{R}+N_{W}}
$$

The dilution is related to the tagging and mistagging probabilities by

$$
D \equiv 2 \cdot P_{t a g}-1 \equiv P_{t a g}-P_{\text {mistag }} \equiv 1-2 \cdot P_{\text {mistag }}
$$

A dilution of one corresponds to tagging that is always right; a dilution of negative one to tagging that is always wrong; and a dilution of zero to tagging that is right as often as it is wrong, which is to say, useless. A zero dilution is the worst one can do - consider that a flavor-tagger that is always wrong can be made into one that is always right by a redefinition of what one means by "right" and "wrong".

High efficiency and dilution are both desired of a flavor-tagger, but most often, one can only be improved at the expense of the other. The quantity which must be maximized to give optimum tagging performance is $\epsilon D^{2}$. In a sample of $N$ events containing a physical mixing asymmetry $A_{\text {true }}$, an experiment using an imperfect flavor-tagger will yield a measured asymmetry, $A_{\text {meas }}$, which in the general case will 
have a different value than $A_{\text {true }}$. $A_{\text {true }}$ is related to $A_{\text {meas }}$ by

$$
A_{\text {true }}=\frac{1}{D} A_{\text {meas }}
$$

The error on the measurement is

$$
\sigma_{A_{\text {true }}}^{2}=\frac{1-D^{2} A_{\text {true }}^{2}}{N \epsilon D^{2}}
$$

For sufficiently small values of $D A_{\text {true }}$, the error on $A_{\text {true }}$ scales as $\frac{1}{\sqrt{N \epsilon D^{2}}}$. As Poisson statistics determine that the relative error on a measurement from a distribution of $N$ points should scale as $\frac{\sqrt{N}}{N}=\frac{1}{\sqrt{N}}$, the measurement with an imperfect tagger effectively reduces the number of events by the factor $\epsilon D^{2}$. For this reason, $\epsilon D^{2}$ is called the effective tagging efficiency. The error on $A_{\text {true }}$ will be minimized by maximizing $\epsilon D^{2}$, given that $N$ is fixed by the amount of data collected by the experiment.

This analysis uses three independent flavor-tagging methods (where SLTs count twice, once for electrons and once for muons). Given a set of flavor-tags of varying $P_{t a g}$ (and thus $\left.D\right), \epsilon D^{2}$ is maximized by weighting each event by $P_{t a g}$. In this scenario, each event contributes to reducing the measurement error by a factor $D^{2}$ and the total $\epsilon D^{2}$ will be

$$
\frac{1}{N} \sum_{i=1}^{n} D_{i}^{2}
$$

The studies of the flavor-tagging methods, presented in the sections to follow, are directed towards providing an event-by-event dilution. Converted to $P_{t a g}$, it will be used in the fit for $\Delta m_{d}$ (described in Chapter 8), thereby reducing the statistical error on $\Delta m_{d}$ by maximizing the effective flavor-tagging efficiency.

Derivations of the equations concerning $D$ and $\epsilon D^{2}$ are presented in Appendix A, 
along with additional relevant discussion.

\subsection{Soft Lepton Flavor-Tagging}

The goal of soft lepton flavor-tagging is to locate electrons and muons from the semileptonic decay of the second $b$ in the event; the charge of these leptons identifies the $b$ flavor just as trigger leptons do. The SLT tagging efficiency is limited by the semileptonic branching ratio (approximately 10\% each for electrons and muons) and the geometric acceptance for the second $b$ (approximately 40\%). Soft leptons are so named because they usually have low $p_{\mathrm{t}}$ and can lie close to other particles in the event. Since backgrounds increase as $p_{\mathrm{t}}$ decreases, stricter identification criteria than those used for trigger leptons must be employed. In the case of soft muons, as there is no Level 3 trigger requirement, more muon types can be used by the tagger.

Soft lepton tagging algorithms were first developed for use in the CDF top analyses $[54,55,63]$, where they were used to identify jets as $b$ jets. As the background rejection needs of the top analyses and this analysis are substantially different, it was determined that the quality cuts applied by the top soft muon tagger were too severe. Therefore, an alternative tagger was developed for this analysis, which blends the essential features of the top soft muon algorithm with more liberal requirements. The soft electron tagger was also slightly modified, including new corrections to the $d E / d x$ response of tracks.

\subsubsection{Soft Electron Identification Algorithm}

The electron identification algorithm employed both by the trigger and by production is efficient only for well-isolated, high-energy electrons. In particular, the cuts placed on the energy sharing and hadronic-to-electromagnetic energy ratio disfavor 
electrons which pass close to hadrons in $\eta$ - $\phi$ space. This algorithm is rather inefficient when searching for an electron in the second $b$ jet, as it typically has a lower energy, and may not be well-isolated.

To increase electron-finding efficiency, CDF has developed a track-based, rather than calorimeter-based, electron identification algorithm. This algorithm was first used in CDF's top quark production evidence publication [54]. This analysis uses a slightly modified version of this algorithm; most of the differences are minor. For example, the conversion-removal procedure is changed to be the same as that used by this analysis for trigger electrons, which gives nearly identical results to the procedure in the top analysis. The only major change was in the soft electron candidates' $d E / d x$ handling: new improvements to the $d E / d x$ corrections (see Appendix C) were developed after the publication of the top discovery. After the new corrections are applied, the $d E / d x$ cut is tuned to be $90 \%$ efficient for electrons, which rejects approximately $50 \%$ of the background from hadrons.

All tracks, with the exception of the trigger lepton, are evaluated to see if they pass the soft electron cuts (listed in Table 7.1). The tracks must pass standard track quality requirements, be within $5 \mathrm{~cm}$ in $z$ of the primary vertex, and have $p_{\mathrm{t}}>2 \mathrm{GeV} / \mathrm{c}$. As electron showers are typically much narrower than a single calorimeter tower, the strictest identification requirements are placed on CES and CPR quantities, which directly measure the shower development.

The tracks are projected from the origin to both the CES and the CPR, and must be located away from the edges of those chambers. The track's CES energy is calculated by summing the energy deposited on the five strips $\left(E_{\text {strip }}\right)$ and wires $\left(E_{\text {wire }}\right)$ around the projected track location. The energy resolution is not good (the CES only samples the shower energy at a single depth), and there is also some 
variation of the mean energy with momentum, owing to shower fluctuations for lowmomentum electrons. The minimum energy cuts include a momentum-dependent term to accommodate the variation. The position of the CES cluster is taken from the energy-weighted mean position of the three strips and wires around the projected track location, giving an $x$ and $z$ position in local CES coordinates (the local $x$ axis is in the azimuthal $(\phi)$ direction; the local $z$ axis is parallel to the global $z$ axis). The track-to-cluster matching $(|\Delta x|,|\Delta z|)$ is required to be consistent with the electron hypothesis. The shower shape, determined from a window of seven strips and wires around the track position, must also be consistent with a standard electron shower shape.

The energy deposited in the $\operatorname{CPR}\left(Q_{C P R}\right)$ is calculated by summing the charge on the three wires around the projected track location. The amount of charge deposited is a function of the track's polar angle, as tracks at large $|\cos \theta|$ traverse more of the solenoid's material (and therefore produce more particles in their shower, on average) than do those perpendicular to the beamline. Correspondingly, the cut on $Q_{C P R}$ is given an angular dependence to account for this effect. The cut value is placed at the approximate charge deposited by the equivalent of four minimum ionizing particles, selecting particles which have begun to shower in the material of the solenoid.

The soft electron selection criteria described so far are almost totally isolationindependent. Even the CES and CPR requirements, sensitive to the particle's showers, rely on a narrow enough window around the projected location of the soft electron candidate that they are fairly insensitive to the presence of nearby tracks. Unfortunately, these criteria alone would result in a $2.5 \%$ per track fake rate, which is not acceptable. Additional fake electron rejection is obtained by placing requirements on the central calorimeter-based $E / p$ and $E_{h a d} / E_{e m}$, as is done for 
trigger electrons. Besides the values of the cuts, there is an essential difference: the energies are taken from a single electromagnetic calorimeter tower, unless the track is sufficiently close to a tower boundary in $\eta$, in which case the two neighboring towers are taken together. In contrast, a trigger electron's energy is always taken from the seed tower and its two neighbors. The requirement for the energy-to-momentum ratio is $0.7<E_{e m} / p<1.5$, while the hadronic-to-electromagnetic energy ratio must be $E_{h a d} / E_{e m}<0.1$, a substantially looser requirement than that for trigger electrons. The addition of these cuts reduces the per-track fake rate to $0.3 \%$.

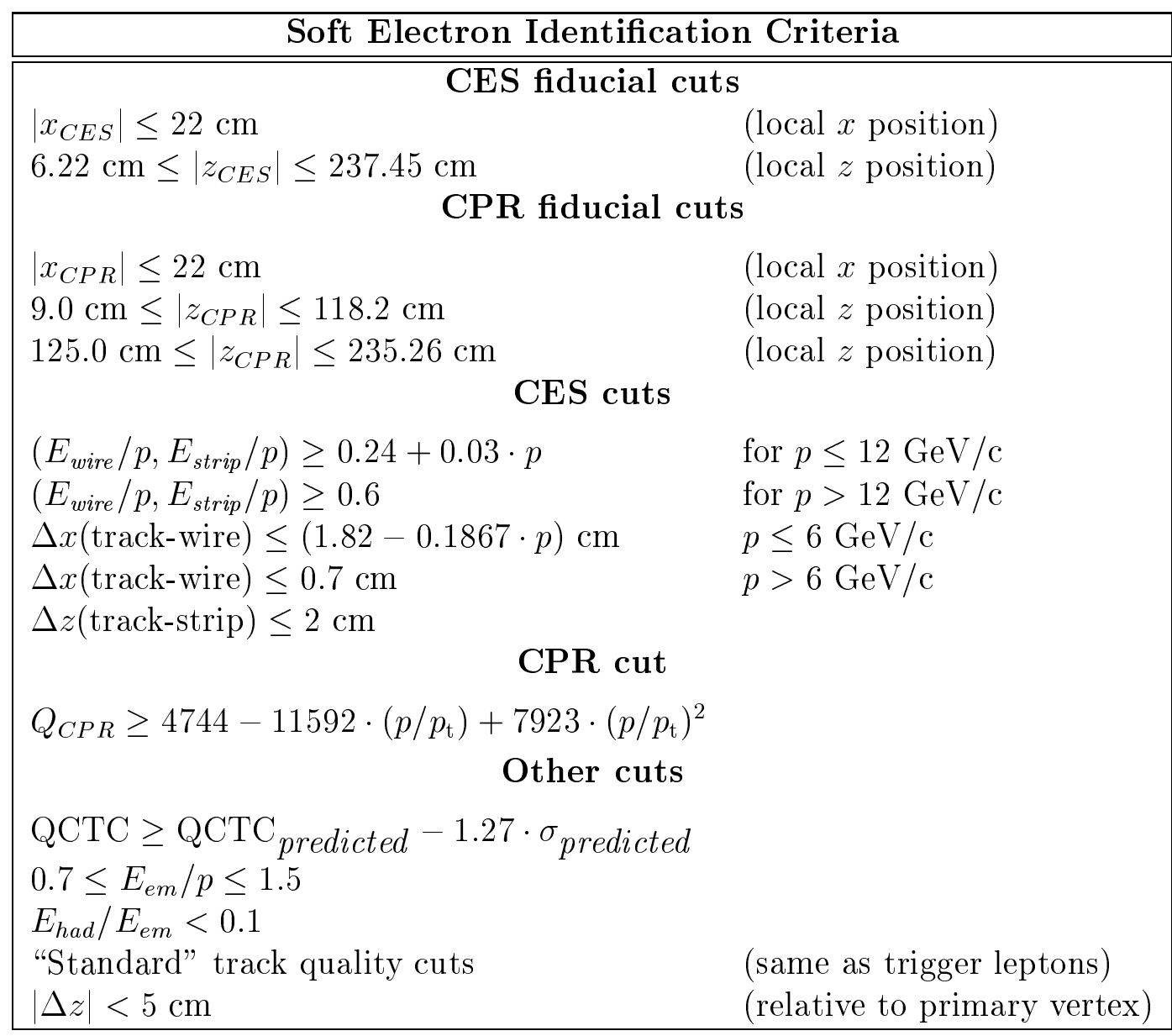

Table 7.1: Soft Electron Identification Criteria.

Local CES and CPR coordinates have the $z$ axis parallel to the CDF global $z$ axis, with $z=0$ at the $\eta=0$ calorimeter boundary; the $x$ axis is in the azimuthal direction, with $x=0$ at the center of the given wedge. Where a cut depends on the track momentum, the units are in $\mathrm{GeV} / \mathrm{c}$. 


\subsubsection{Soft Muon Identification Algorithm}

The muons identified by this tagger will be referred to as projective muons, as the essential feature of the tagger is to extrapolate a track from the CTC outward into the muon chambers, where it is matched to stubs in those chambers. The tagger uses the same software for the projection as the Level 3 trigger and production. Another feature of the tagger is the application of corrections for magnetic field and drift velocity to CMX stubs. These can change the position of the stub, and therefore the track-stub matching $\chi^{2}$. The corrections were not available at the commencement of production; they are not applied to trigger muons, as the $p_{\mathrm{t}}$ requirements of the trigger sharply reduces their necessity.

The Stream B single muon triggers which created the dataset only allowed two types of muons: CMUP and CMX. As there is no trigger requirement for projective muons, more types can be used. Projective muons are of four types: $\mathrm{CMU}^{1}, \mathrm{CMP}$, CMUP, and CMX. As there is essentially no physical overlap between the CMX and the other muon chambers (see Figure 2.9), candidates matched to stubs in the CMX and any other chamber are rejected.

The track projection is used to select soft muons in two ways. If a track definitely went inside a muon chamber, it must be matched to a stub in that chamber. If it definitely missed a chamber, it must not be matched to a stub in that chamber. The definition of definitely depends on the calculated uncertainty of the track's projected location, which is largely due to multiple scattering in the material of the detector. A muon located 3 standard deviations away from a chamber boundary is definitely inside or outside that chamber. A muon which projects too close to a chamber boundary is a "maybe": a stub in that chamber is not required, but if

\footnotetext{
${ }^{1}$ For clarification, a CMU muon is not any muon candidate with a CMU stub, but rather a candidate with only a CMU stub.
} 
present, it is accepted.

The track projection is followed by a set of requirements on the matching between the track and the accepted stubs. The requirements used in the top analyses result in high-quality tag muons, at a great cost in efficiency. As the backgrounds faced by this analysis are much smaller, the matching cuts were loosened, to the values used in the trigger muon selection:

$$
\begin{array}{ll}
\text { CMU: } & \chi_{x}^{2}<9.0, \chi_{z}^{2}<12.0 \\
\text { CMP: } & \chi_{x}^{2}<9.0 \\
\text { CMX: } & \chi_{x}^{2}<9.0, \chi_{z}^{2}<12.0
\end{array}
$$

The muon track must also pass standard track quality cuts and be within $5 \mathrm{~cm}$ in $z$ of the primary vertex.

\subsubsection{Soft Lepton Classification}

The 89,262 trigger electron events contain 3,271 soft electrons and 7,622 soft muons, and the 82,656 trigger muon events contain 3,099 soft electrons and 8,889 soft muons, not counting the trigger lepton (even if it passes the SLT criteria). Soft leptons are partitioned into four classes according to the following kinematic criteria:

- If the invariant mass of the SLT and the trigger lepton $\left(M_{\ell \ell}\right)$ is $\leq 5 \mathrm{GeV} / \mathrm{c}^{2}$, and the SLT is in the same TRKSJT jet as the trigger lepton, the candidate is said to be in the trigger jet. These candidates are likely from double semileptonic $b$ decay $(b \rightarrow c \ell ; c \rightarrow \ell)$. To repeat, the trigger lepton is itself excluded from this consideration.

- If $M_{\ell \ell}>5 \mathrm{GeV} / \mathrm{c}^{2}$ and the SLT is not in the same TRKSJT jet as the trigger lepton, the SLT is said not to be in the trigger jet. These candidates likely come from the semileptonic decay of the second $b$ in the event; colloquially, these SLTs are also referred to as "tag leptons". 
- Candidates with $M_{\ell \ell}>5 \mathrm{GeV} / \mathrm{c}^{2}$ that are in the same jet as the trigger lepton are excluded from consideration.

- Candidates with $M_{\ell \ell} \leq 5 \mathrm{GeV} / \mathrm{c}^{2}$ that are not in the same jet as the trigger lepton are also excluded.

The number of soft electrons and muons in each of the four categories is shown in Table 7.2. A study of the $b \bar{b}$ Monte Carlo samples is the motivation for excluding leptons from the last two classes. Of those leptons which are not decay products of the same $b$ hadron as the trigger lepton, almost none are closer than $\Delta R=0.8$ to the trigger lepton or have $M_{\ell \ell} \leq 5$, as shown in Figure 7.1. The exclusion of those which $d o$ meet these requirements in the data samples rejects only backgrounds to real semileptonic $b$ decay.

\section{SLTs in the Trigger Jet}

The number of soft electrons in the trigger jet, in bins of $p_{\mathrm{t}}$, is shown in Table 7.3. The corresponding numbers for soft muons are shown in Tables 7.4 and 7.5. The taggers show a large excess of opposite sign over same sign leptons, as expected from double-sequential decays. Figures 7.2 and 7.3 show the invariant mass distribution of soft leptons relative to the trigger lepton. Note that the trigger jet SLTs only occupy the mass region from 0 to $5 \mathrm{GeV} / \mathrm{c}^{2}$; the entries above $5 \mathrm{GeV} / \mathrm{c}^{2}$ are the tag leptons. A $J / \psi$ peak is clearly evident in the cases where the trigger and soft leptons are the same flavor, as expected. 


\begin{tabular}{|r|r|r|}
\hline Soft electrons in trigger electron data \\
\hline & $M_{\ell \ell}<5$ & $M_{\ell \ell}<5$ \\
\hline in trigger jet & 1686 & 58 \\
not in trigger jet & 36 & 1491 \\
\hline
\end{tabular}

\begin{tabular}{|r|r|r|}
\hline \multicolumn{3}{|c|}{ Soft electrons in trigger muon data } \\
\hline in trigger jet & $M_{\ell \ell}<5$ & $M_{\ell \ell}<5$ \\
not in trigger jet & 1557 & 71 \\
& 35 & 1436 \\
\hline
\end{tabular}

\begin{tabular}{|c|c|c|}
\hline \multicolumn{3}{|c|}{ Soft muons in trigger electron data } \\
\hline & $M_{\ell \ell}<5$ & $M_{\ell \ell}<5$ \\
\hline in trigger jet & 3893 & 98 \\
\hline not in trigger jet & 93 & 3538 \\
\hline \multicolumn{3}{|c|}{ Soft muons in trigger muon data } \\
\hline & $M_{\ell \ell}<5$ & $M_{\ell \ell}<5$ \\
\hline in trigger jet & 5297 & 132 \\
\hline not in trigger jet & 89 & 3371 \\
\hline
\end{tabular}

Table 7.2: Numbers of soft electrons and muons in each of the four classifications, as described in the text.
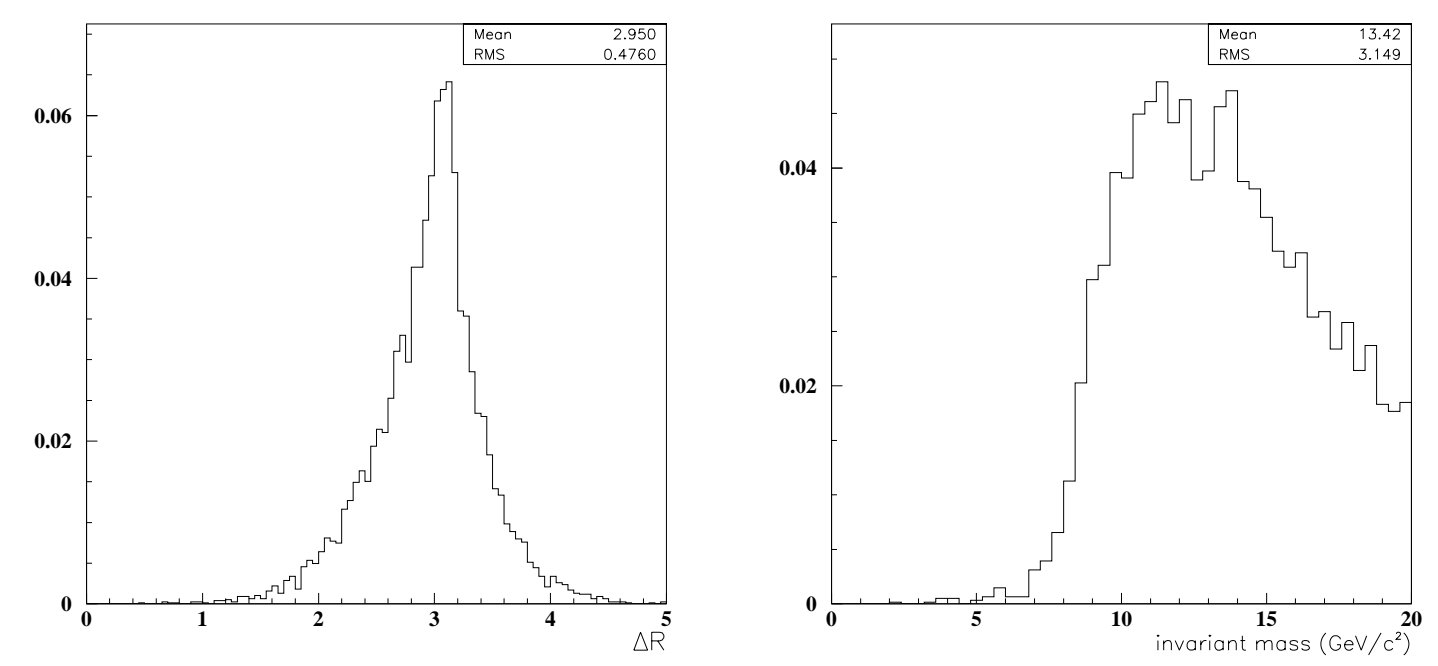

Figure 7.1: The $\Delta R$ (left) and invariant mass (right), relative to the trigger lepton, of leptons that do not share the same $b$ hadron parent as the trigger lepton. The events are taken from the $b \bar{b}$ Monte Carlo samples. Invariant masses larger than $20 \mathrm{GeV} / \mathrm{c}^{2}$ are not shown. Real leptons from the semileptonic decay of the second $b$ clearly do not typically have $\Delta R<0.8$ and $M_{\ell \ell} \leq 5.0 \mathrm{GeV} / \mathrm{c}^{2}$. 


\begin{tabular}{|c|c|c|c|c|c|}
\hline \multicolumn{6}{|c|}{ Soft Electrons in the Trigger Jet } \\
\hline \hline $\begin{array}{c}p_{\mathrm{t}} \\
(\mathrm{GeV} / \mathrm{c})\end{array}$ & \#OS & \#SS & $\epsilon(\%)$ & $\mathrm{D}(\%)$ & $\epsilon D^{2}(\%)$ \\
\hline \hline \multicolumn{6}{|c|}{ Trigger Electron Data } \\
\hline $2.0-2.5$ & 322 & 59 & $0.427 \pm 0.022$ & $69.0 \pm 3.7$ & $0.203 \pm 0.024$ \\
$2.5-3.0$ & 269 & 35 & $0.341 \pm 0.020$ & $77.0 \pm 3.7$ & $0.202 \pm 0.022$ \\
$3.0-4.0$ & 316 & 46 & $0.406 \pm 0.021$ & $74.6 \pm 3.5$ & $0.226 \pm 0.024$ \\
$4.0+$ & 576 & 63 & $0.716 \pm 0.028$ & $80.3 \pm 2.4$ & $0.461 \pm 0.033$ \\
\hline \hline \multicolumn{6}{|c|}{ Trigger Muon Data } \\
\hline $2.0-2.5$ & 251 & 84 & $0.405 \pm 0.022$ & $49.9 \pm 4.7$ & $0.101 \pm 0.020$ \\
$2.5-3.0$ & 175 & 40 & $0.260 \pm 0.018$ & $62.8 \pm 5.3$ & $0.103 \pm 0.019$ \\
$3.0-4.0$ & 283 & 80 & $0.439 \pm 0.023$ & $55.9 \pm 4.4$ & $0.137 \pm 0.023$ \\
$4.0+$ & 553 & 91 & $0.779 \pm 0.031$ & $71.7 \pm 2.7$ & $0.401 \pm 0.035$ \\
\hline
\end{tabular}

Table 7.3: Number of same and opposite sign charge electrons in the trigger lepton jet, as a function of $p_{\mathrm{t}}$. "Dilutions" have not been corrected for background or mixing in the trigger jet, and are shown for informational purposes only.

\begin{tabular}{|l|l|r|r|c|c|c|}
\hline \multicolumn{7}{|c|}{ Projective Muons in the Trigger Jet } \\
\hline \multicolumn{7}{|c|}{ Trigger Electron Data } \\
\hline \hline $\begin{array}{c}p_{\mathrm{t}} \\
(\mathrm{GeV} / \mathrm{c})\end{array}$ & $\#$ OS & \#SS & $\epsilon(\%)$ & $\mathrm{D}(\%)$ & $\epsilon D^{2}(\%)$ \\
\hline$C M U$ & $2.0-2.5$ & 625 & 180 & $0.902 \pm 0.032$ & $55.3 \pm 2.9$ & $0.276 \pm 0.031$ \\
$C M U$ & $2.5-3.0$ & 519 & 143 & $0.742 \pm 0.029$ & $56.8 \pm 3.2$ & $0.239 \pm 0.029$ \\
$C M U$ & $3.0-4.0$ & 167 & 56 & $0.250 \pm 0.017$ & $49.8 \pm 5.8$ & $0.062 \pm 0.015$ \\
$C M U$ & $4.0+$ & 248 & 76 & $0.363 \pm 0.020$ & $53.1 \pm 4.7$ & $0.102 \pm 0.019$ \\
\hline$C M P$ & $3.0-4.0$ & 79 & 18 & $0.109 \pm 0.011$ & $62.9 \pm 7.9$ & $0.043 \pm 0.012$ \\
$C M P$ & $4.0+$ & 105 & 20 & $0.140 \pm 0.013$ & $68.0 \pm 6.6$ & $0.065 \pm 0.014$ \\
\hline$C M U P$ & $3.0-4.0$ & 402 & 57 & $0.514 \pm 0.024$ & $75.2 \pm 3.1$ & $0.291 \pm 0.027$ \\
$C M U P$ & $4.0+$ & 495 & 82 & $0.646 \pm 0.027$ & $71.6 \pm 2.9$ & $0.331 \pm 0.030$ \\
\hline$C M X$ & $2.0-2.5$ & 100 & 20 & $0.134 \pm 0.012$ & $66.7 \pm 6.8$ & $0.060 \pm 0.013$ \\
$C M X$ & $2.5-3.0$ & 94 & 24 & $0.132 \pm 0.012$ & $59.3 \pm 7.4$ & $0.047 \pm 0.012$ \\
$C M X$ & $3.0-4.0$ & 130 & 31 & $0.180 \pm 0.014$ & $61.5 \pm 6.2$ & $0.068 \pm 0.015$ \\
$C M X$ & $4.0+$ & 186 & 36 & $0.249 \pm 0.017$ & $67.6 \pm 4.9$ & $0.114 \pm 0.018$ \\
\hline
\end{tabular}

Table 7.4: Number of same and opposite sign projective muons in trigger electron jets, as a function of $p_{\mathrm{t}}$. "Dilutions" have not been corrected for background or mixing in the trigger jet, and are shown for informational purposes only. 


\begin{tabular}{|l|l|r|r|c|c|c|}
\hline \multicolumn{7}{|c|}{$\begin{array}{c}\text { Projective Muons in the Trigger Jet } \\
\text { Trigger Muon Data }\end{array}$} \\
\hline \hline Type & $\begin{array}{c}p_{\mathrm{t}} \\
(\mathrm{GeV} / \mathrm{c})\end{array}$ & \#OS & $\# \mathrm{SS}$ & $\epsilon(\%)$ & $\mathrm{D}(\%)$ & $\epsilon D^{2}(\%)$ \\
& & & & & \\
\hline$C M U$ & $2.0-2.5$ & 797 & 266 & $1.191 \pm 0.037$ & $50.0 \pm 2.7$ & $0.297 \pm 0.033$ \\
$C M U$ & $2.5-3.0$ & 612 & 213 & $0.924 \pm 0.032$ & $48.4 \pm 3.0$ & $0.216 \pm 0.028$ \\
$C M U$ & $3.0-4.0$ & 188 & 80 & $0.300 \pm 0.018$ & $40.3 \pm 5.6$ & $0.049 \pm 0.014$ \\
$C M U$ & $4.0+$ & 332 & 100 & $0.484 \pm 0.023$ & $53.7 \pm 4.1$ & $0.140 \pm 0.022$ \\
\hline$C M P$ & $3.0-4.0$ & 159 & 65 & $0.251 \pm 0.017$ & $42.0 \pm 6.1$ & $0.044 \pm 0.013$ \\
$C M P$ & $4.0+$ & 363 & 122 & $0.543 \pm 0.025$ & $49.7 \pm 3.9$ & $0.134 \pm 0.022$ \\
\hline$C M U P$ & $3.0-4.0$ & 482 & 88 & $0.639 \pm 0.027$ & $69.1 \pm 3.0$ & $0.305 \pm 0.030$ \\
$C M U P$ & $4.0+$ & 962 & 137 & $1.231 \pm 0.037$ & $75.1 \pm 2.0$ & $0.694 \pm 0.042$ \\
\hline$C M X$ & $2.0-2.5$ & 49 & 16 & $0.073 \pm 0.009$ & $50.8 \pm 10.7$ & $0.019 \pm 0.008$ \\
$C M X$ & $2.5-3.0$ & 49 & 9 & $0.065 \pm 0.009$ & $69.0 \pm 9.5$ & $0.031 \pm 0.009$ \\
$C M X$ & $3.0-4.0$ & 70 & 21 & $0.102 \pm 0.011$ & $53.8 \pm 8.8$ & $0.030 \pm 0.010$ \\
$C M X$ & $4.0+$ & 95 & 22 & $0.131 \pm 0.012$ & $62.4 \pm 7.2$ & $0.051 \pm 0.013$ \\
\hline
\end{tabular}

Table 7.5: Number of same and opposite sign projective muons in trigger muon jets, as a function of $p_{\mathrm{t}}$. "Dilutions" have not been corrected for background or mixing in the trigger jet, and are shown for informational purposes only. 

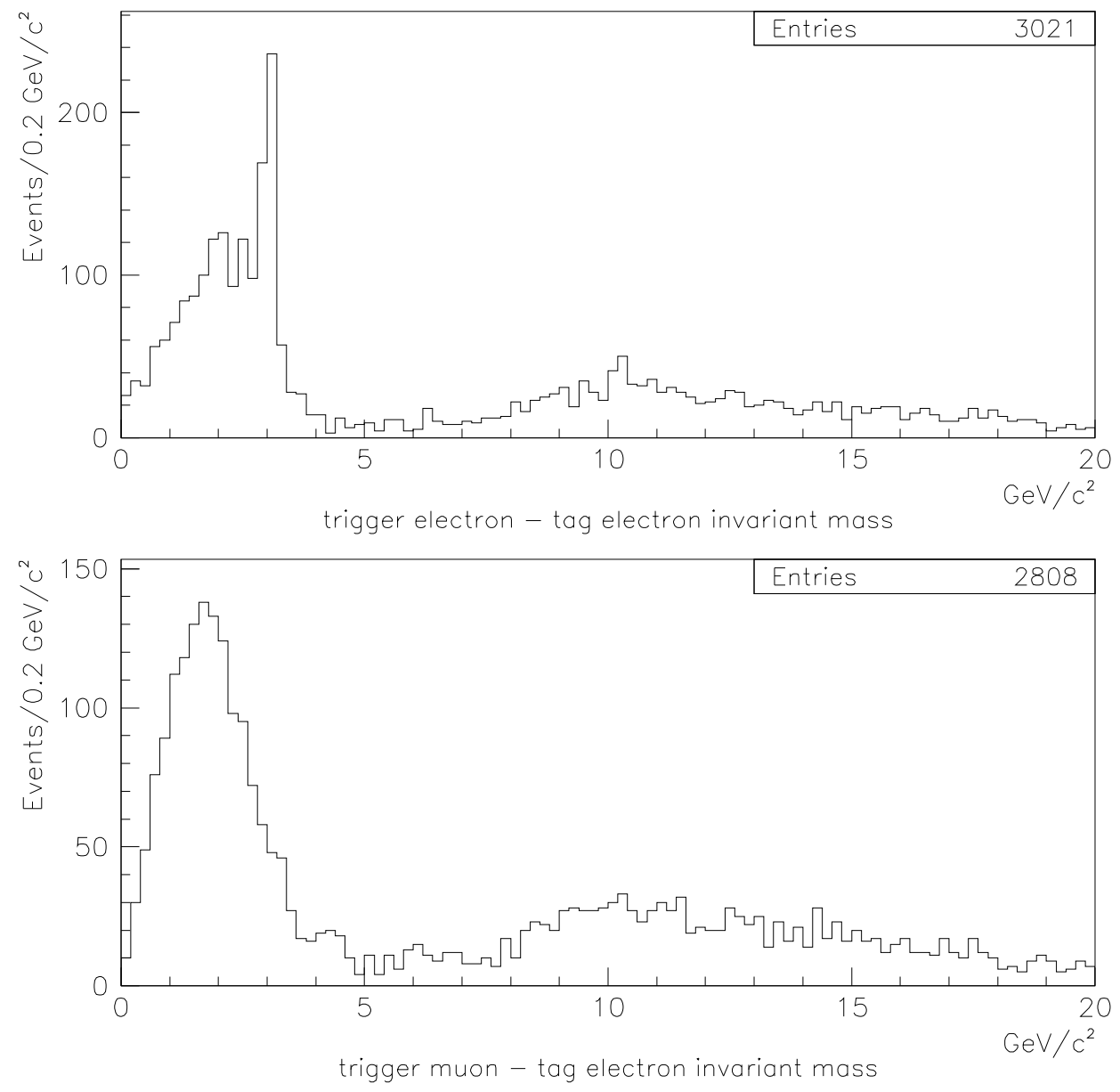

Figure 7.2: The invariant mass distribution of soft electrons relative to the trigger lepton for trigger electron (top) and trigger muon (bottom) data. Electrons with invariant mass less than $5 \mathrm{GeV} / \mathrm{c}^{2}$ are trigger jet electrons, and those with greater than $5 \mathrm{GeV} / \mathrm{c}^{2}$ are tag electrons. Masses greater than $20 \mathrm{GeV} / \mathrm{c}^{2}$ are not shown. A clear $J / \psi$ peak can be seen in the trigger electron data. 

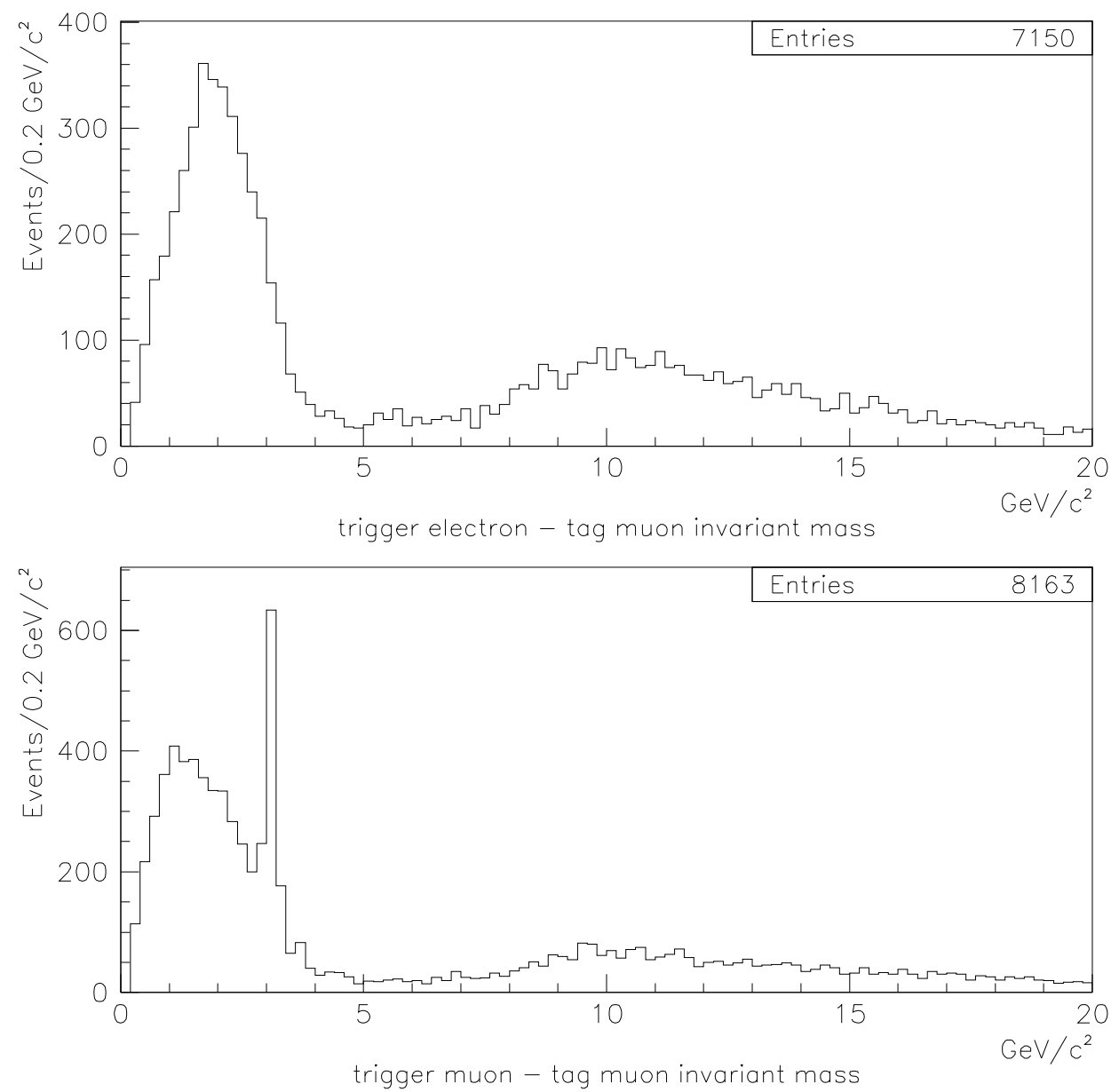

Figure 7.3: The invariant mass distribution of soft muons relative to the trigger lepton for trigger electron (top) and trigger muon (bottom) data. Muons with invariant mass less than $5 \mathrm{GeV} / \mathrm{c}^{2}$ are trigger jet muons, and those with greater than $5 \mathrm{GeV} / \mathrm{c}^{2}$ are tag muons. Masses greater than $20 \mathrm{GeV} / \mathrm{c}^{2}$ are not shown. A clear $J / \psi$ peak can be seen in the trigger muon data. 


\subsubsection{Tag Leptons and Raw Dilution Parametrization}

As mentioned in Section 7.1, and discussed in detail in Appendix A, in the case where one has a set of tags of varying dilution, the best way to use the tagging information (and therefore maximize $\epsilon D^{2}$ ) is to subdivide the set into as many subsamples as possible and sum the $\epsilon D^{2}$ from each. In the perfect case where one knew the dilution of each individual tag, the maximum $\epsilon D^{2}$ would be obtained from summing the $D^{2}$ of each tag, rather than calculating an average $D$ for the set of tags. This can be realized in practice by supplying the event-by-event probability that the flavor-tag is correct (calculated from the dilution) to the likelihood function used to fit for $\Delta m_{d}$. To this end, the raw dilution is measured in bins ${ }^{2}$ of different kinematic variables to determine which yields the largest $\sum \epsilon D^{2}$. The dilution is then parametrized as a function of that variable.

For SLT flavor-tagging, three kinematic variables of the soft lepton are studied: its $p_{\mathrm{t}}, p_{\mathrm{t}}^{\mathrm{rel}}$, and a combination of the two, called $p_{\mathrm{t}}^{\text {comb }}$. The soft lepton $p_{\mathrm{t}}^{\mathrm{rel}}$ is calculated using Equation 6.1, just as for trigger leptons; the only difference is that a TRKSJT clustering cone size of $\Delta R=0.8$ and a minimum $N_{\text {trk }}^{\text {jet }} \geq 3$ is used, reflecting the softer $p_{\mathrm{t}}$ spectrum of the second $b$ jet. Though the classification scheme of Section 7.2.3 made no explicit requirement that a tag lepton be in a jet, the $p_{\mathrm{t}}$ and track quality requirements placed on the SLTs will ensure that TRKSJT clusters them in a jet (see Section 4.5).

Some leptons, sufficiently isolated from other tracks, will be in jets with $N_{\text {trk }}^{\text {jet }}=1$ or 2. By the above requirements, they will have an undefined $p_{\mathrm{t}}^{\text {rel }}$. Because of the different kinematics of direct and sequential $b$ decay, these leptons are much more likely to be from direct $b$ decay, and have a high dilution. "No $p_{\mathrm{t}}^{\text {rel" }}$ is used as another $p_{\mathrm{t}}^{\text {rel }}$ bin in the summation of $\epsilon D^{2}$; the raw dilution of these tags is tabulated

\footnotetext{
${ }^{2}$ Any bin with a negative dilution subtracts from the total $\epsilon D^{2}$.
} 
separately, as measured, and not included in a functional parametrization of the raw dilution.

$p_{\mathrm{t}}^{\mathrm{comb}}$, a combination of $p_{\mathrm{t}}$ and $p_{\mathrm{t}}^{\mathrm{rel}}$, is formed to learn if it separates low- from high-dilution tags better than either quantity alone. $p_{\mathrm{t}}^{\mathrm{comb}}$ is defined as

$$
p_{\mathrm{t}}^{\mathrm{comb}}=\sqrt{\left(\frac{p_{\mathrm{t}}}{5}\right)^{2}+\left(p_{\mathrm{t}}^{\mathrm{rel}}\right)^{2}}
$$

To prevent the $p_{\mathrm{t}}$ from dominating the combination, it is scaled by the approximate $b$ hadron mass; a study shows that the dilution versus $p_{\mathrm{t}}^{\text {comb }}$ is not particularly sensitive to the scale factor for values within the range of $\sim 3-9$. Smaller or larger values drive the dilution towards the results from $p_{\mathrm{t}}$ or $p_{\mathrm{t}}^{\text {rel }}$ alone. Those leptons with no $p_{\mathrm{t}}^{\text {rel }}$ also have no $p_{\mathrm{t}}^{\mathrm{comb}}$.

The number of opposite- and same-sign soft lepton tags (not in the trigger jet) versus $p_{\mathrm{t}}$, as well as the efficiency, dilution, and $\epsilon D^{2}$, is shown numerically in Tables 7.6-7.9 and graphically in Figures 7.4-7.7. Results for the trigger electron and muon samples are shown separately. As the number of CMP muons is small, they are combined with the CMUP muons in the figures; they are left separate in the tables for illustration. It is worth repeating that the dilutions shown are the raw dilutions, which are smaller than the true flavor-tagging dilutions - they have not been corrected for backgrounds or mixing in the trigger jet.

The total $\epsilon D^{2}$ for soft electron flavor-tagging from the sum over bins of $p_{\mathrm{t}}^{\mathrm{rel}}$ and $p_{\mathrm{t}}^{\text {comb }}$ is also shown in Tables 7.6 and 7.7; the same information for soft muon flavor-tagging is shown in Tables 7.10 and 7.11. CMP and CMUP muons are again combined. The efficiency, dilution, and $\epsilon D^{2}$ versus $p_{\mathrm{t}}^{\text {rel }}$ and $p_{\mathrm{t}}^{\text {comb }}$ is shown graphically in Figures 7.8-7.15. 


\begin{tabular}{|c|c|c|c|c|c|}
\hline \multicolumn{6}{|c|}{ Tag Electrons in Electron Data } \\
\hline $\begin{array}{c}p_{\mathrm{t}} \\
(\mathrm{GeV} / \mathrm{c})\end{array}$ & \#OS & \#SS & $\epsilon(\%)$ & $\mathrm{D}(\%)$ & $\epsilon D^{2}(\%)$ \\
\hline \multicolumn{6}{|c|}{ Versus $p_{t}$} \\
\hline $2.0-2.5$ & 177 & 141 & $0.356 \pm 0.020$ & $11.3 \pm 5.6$ & $0.005 \pm 0.005$ \\
\hline $2.5-3.0$ & 143 & 97 & $0.269 \pm 0.017$ & $19.2 \pm 6.3$ & $0.010 \pm 0.007$ \\
\hline $3.0-4.0$ & 164 & 122 & $0.320 \pm 0.019$ & $14.7 \pm 5.8$ & $0.007 \pm 0.006$ \\
\hline $4.0+$ & 428 & 219 & $0.725 \pm 0.028$ & $32.3 \pm 3.7$ & $0.076 \pm 0.018$ \\
\hline Sum : & 912 & 579 & $1.670 \pm 0.043$ & - & $0.098 \pm 0.020$ \\
\hline \multicolumn{6}{|c|}{ Integrated Over $p_{t}^{\text {rel }}$} \\
\hline$p_{\mathrm{t}}^{\mathrm{rel}}$ & 792 & 535 & $1.487 \pm 0.041$ & - & $0.087 \pm 0.018$ \\
\hline \multicolumn{6}{|c|}{ Integrated Over $\mathrm{p}_{\mathrm{t}}^{\mathrm{comb}}$} \\
\hline$p_{\mathrm{t}}^{\mathrm{comb}}$ & 792 & 535 & $1.487 \pm 0.041$ & - & $0.087 \pm 0.019$ \\
\hline \multicolumn{6}{|c|}{ Electrons without $p_{t}^{\text {rel }}$} \\
\hline no $p_{t}^{\text {rel }}$ & 120 & 44 & $0.184 \pm 0.014$ & $46.3 \pm 6.9$ & $0.039 \pm 0.012$ \\
\hline Total $p_{t}^{\text {rel }}$ & 912 & $\overline{579}$ & $1.670 \pm 0.043$ & $\overline{-1}$ & $0.126 \pm 0.022$ \\
\hline Total $p_{t}^{\text {comb }}$ & 912 & 579 & $1.670 \pm 0.043$ & - & $0.126 \pm 0.023$ \\
\hline
\end{tabular}

Table 7.6: Tag electrons in trigger electron data, in bins of $p_{\mathrm{t}}$, and integrated over bins of $p_{\mathrm{t}}^{\text {rel }}$ and $p_{\mathrm{t}}^{\text {comb }}$. Dilutions have not been corrected for background or mixing in the trigger jet.
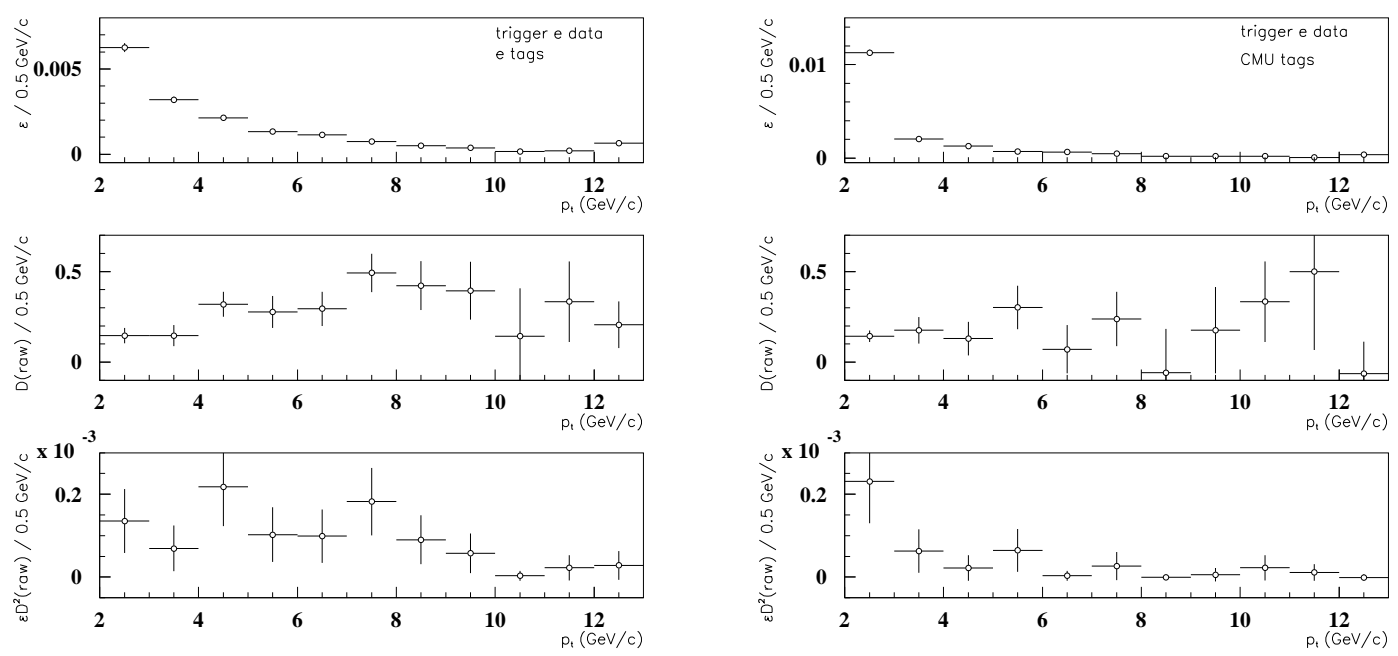

Figure 7.4: Top to bottom: efficiency, dilution, and $\epsilon D^{2}$ versus $p_{\mathrm{t}}$ for soft electron tags (left) and projective CMU tags (right) in trigger electron data. The rightmost bin is the overflow bin. Dilutions have not been corrected for background or mixing in the trigger jet. 


\begin{tabular}{|c|c|c|c|c|c|}
\hline \multicolumn{6}{|c|}{ Tag Electrons in Muon Data } \\
\hline $\begin{array}{c}p_{\mathrm{t}} \\
(\mathrm{GeV} / \mathrm{c}) \\
\end{array}$ & $\# \mathrm{OS}$ & $\# \mathrm{SS}$ & $\epsilon(\%)$ & $\mathrm{D}(\%)$ & $\epsilon D^{2}(\%)$ \\
\hline \multicolumn{6}{|c|}{ Versus $p_{t}$} \\
\hline $2.0-2.5$ & 168 & 140 & $0.373 \pm 0.021$ & $9.1 \pm 5.7$ & $0.003 \pm 0.004$ \\
\hline $2.5-3.0$ & 138 & 108 & $0.298 \pm 0.019$ & $12.2 \pm 6.3$ & $0.004 \pm 0.005$ \\
\hline $3.0-4.0$ & 147 & 118 & $0.321 \pm 0.020$ & $10.9 \pm 6.1$ & $0.004 \pm 0.004$ \\
\hline $4.0+$ & 374 & 243 & $0.746 \pm 0.030$ & $21.2 \pm 3.9$ & $0.034 \pm 0.013$ \\
\hline Sum : & 827 & 609 & $1.737 \pm 0.046$ & - & $0.045 \pm 0.015$ \\
\hline \multicolumn{6}{|c|}{ Integrated Over $p_{t}^{\text {rel }}$} \\
\hline$p_{\mathrm{t}}^{\mathrm{rel}}$ & 752 & 546 & $1.570 \pm 0.044$ & - & $0.068 \pm 0.017$ \\
\hline \multicolumn{6}{|c|}{ Integrated Over $p_{t}^{\text {comb }}$} \\
\hline$p_{\mathrm{t}}^{\mathrm{comb}}$ & 752 & 546 & $1.570 \pm 0.044$ & - & $0.077 \pm 0.018$ \\
\hline \multicolumn{6}{|c|}{ Electrons without $p_{t}^{\text {rel }}$} \\
\hline no $p_{t}^{\text {rel }}$ & 75 & 63 & $0.167 \pm 0.014$ & $8.7 \pm 8.5$ & $0.001 \pm 0.003$ \\
\hline Total $p_{t}^{\text {rel }}$ & 827 & 609 & $1.737 \pm 0.046$ & - & $0.069 \pm 0.017$ \\
\hline Total $p_{t}^{\text {comb }}$ & 827 & 609 & $1.737 \pm 0.046$ & - & $0.078 \pm 0.018$ \\
\hline
\end{tabular}

Table 7.7: Tag electrons in trigger muon data, in bins of $p_{\mathrm{t}}$, and integrated over bins of $p_{\mathrm{t}}^{\text {rel }}$ and $p_{\mathrm{t}}^{\text {comb }}$. Dilutions have not been corrected for background or mixing in the trigger jet.
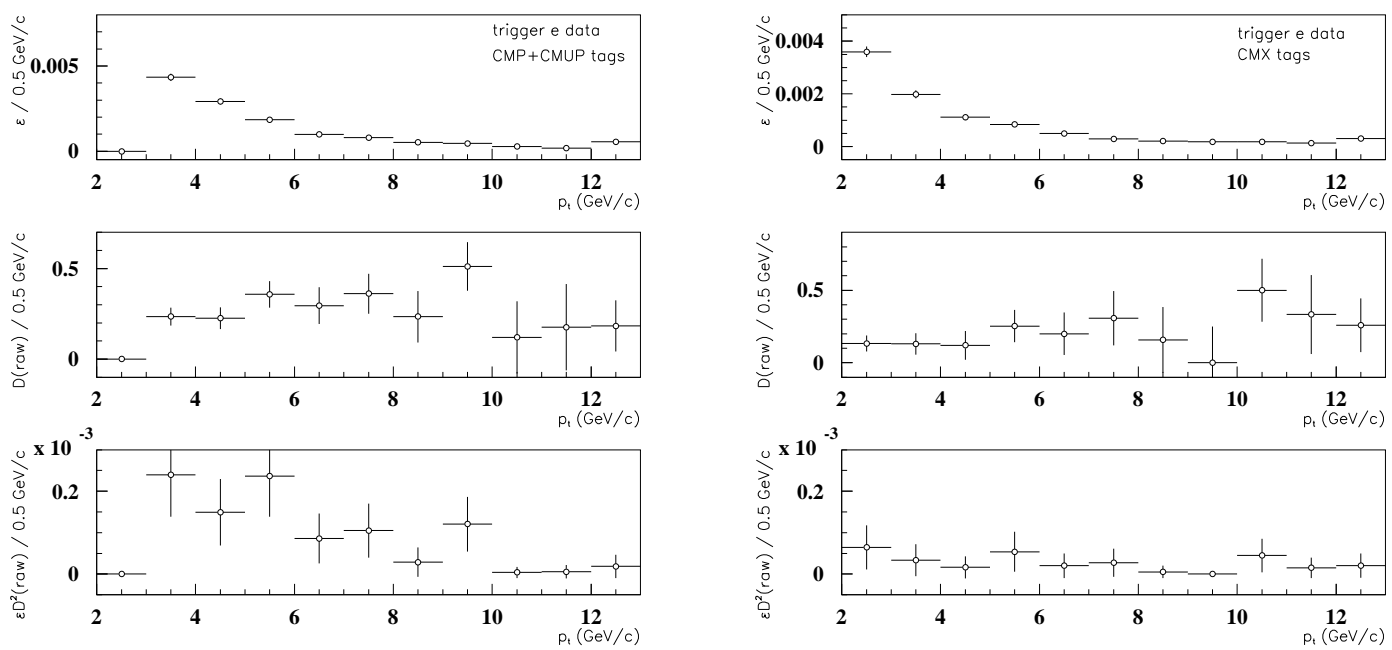

Figure 7.5: Top to bottom: efficiency, dilution, and $\epsilon D^{2}$ versus $p_{\mathrm{t}}$ for projective CMP and CMUP tags (left) and projective CMX tags (right) in trigger electron data. The rightmost bin is the overflow bin. Dilutions have not been corrected for background or mixing in the trigger jet. 


\begin{tabular}{|l|l|r|r|c|r|c|}
\hline \multicolumn{7}{|c|}{ Tag Muons in Electron Data } \\
Projective Cuts \\
\hline Type & $\begin{array}{c}p_{\mathrm{t}} \\
(\mathrm{GeV} / \mathrm{c})\end{array}$ & \#OS & \#SS & $\epsilon(\%)$ & $\mathrm{D}(\%)$ & $\epsilon D^{2}(\%)$ \\
\hline \hline \multicolumn{7}{|c|}{ Projective Cuts } \\
\hline$C M U$ & $2.0-2.5$ & 293 & 243 & $0.600 \pm 0.026$ & $9.3 \pm 4.3$ & $0.005 \pm 0.005$ \\
$C M U$ & $2.5-3.0$ & 282 & 188 & $0.527 \pm 0.024$ & $20.0 \pm 4.5$ & $0.021 \pm 0.010$ \\
$C M U$ & $3.0-4.0$ & 107 & 75 & $0.204 \pm 0.015$ & $17.6 \pm 7.3$ & $0.006 \pm 0.005$ \\
$C M U$ & $4.0+$ & 210 & 154 & $0.408 \pm 0.021$ & $15.4 \pm 5.2$ & $0.010 \pm 0.007$ \\
\hline$C M P$ & $3.0-4.0$ & 31 & 25 & $0.063 \pm 0.008$ & $10.7 \pm 13.3$ & $0.001 \pm 0.002$ \\
$C M P$ & $4.0+$ & 88 & 42 & $0.146 \pm 0.013$ & $35.4 \pm 8.2$ & $0.018 \pm 0.009$ \\
\hline$C M U P$ & $3.0-4.0$ & 208 & 123 & $0.371 \pm 0.020$ & $25.7 \pm 5.3$ & $0.024 \pm 0.010$ \\
$C M U P$ & $4.0+$ & 403 & 232 & $0.711 \pm 0.028$ & $26.9 \pm 3.8$ & $0.052 \pm 0.015$ \\
\hline$C M X$ & $2.0-2.5$ & 84 & 74 & $0.177 \pm 0.014$ & $6.3 \pm 7.9$ & $0.001 \pm 0.002$ \\
$C M X$ & $2.5-3.0$ & 98 & 65 & $0.183 \pm 0.014$ & $20.2 \pm 7.7$ & $0.007 \pm 0.006$ \\
$C M X$ & $3.0-4.0$ & 100 & 77 & $0.198 \pm 0.015$ & $13.0 \pm 7.5$ & $0.003 \pm 0.004$ \\
$C M X$ & $4.0+$ & 203 & 133 & $0.376 \pm 0.021$ & $20.8 \pm 5.3$ & $0.016 \pm 0.008$ \\
\hline Sum : & & 2107 & 1431 & $3.964 \pm 0.067$ & - & $0.164 \pm 0.027$ \\
\hline
\end{tabular}

Table 7.8: Number of same- and opposite-sign projective muons not in the trigger jet, as a function of $p_{\mathrm{t}}$, in trigger electron data. Dilutions have not been corrected for background or mixing in the trigger jet.
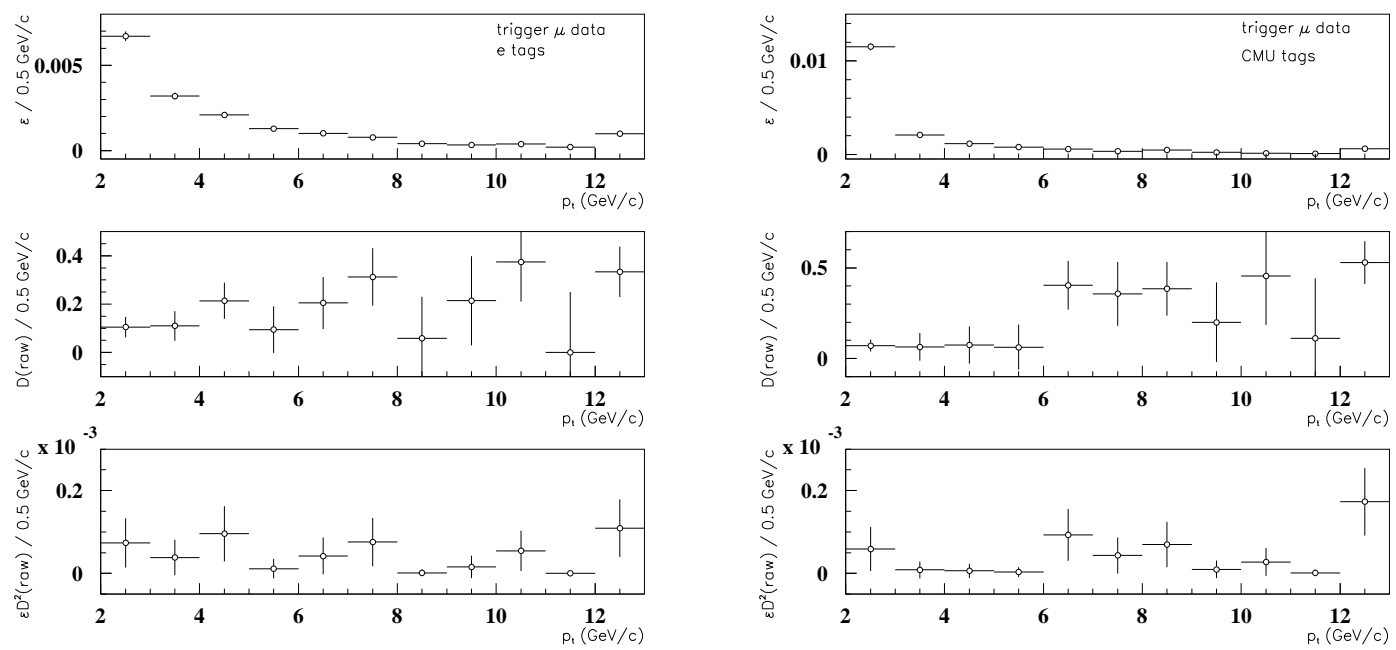

Figure 7.6: Top to bottom: efficiency, dilution, and $\epsilon D^{2}$ versus $p_{\mathrm{t}}$ for soft electron tags (left) and projective CMU tags (right) in trigger muon data. The rightmost bin is the overflow bin. Dilutions have not been corrected for background or mixing in the trigger jet. 


\begin{tabular}{|l|l|r|r|c|r|r|}
\hline \multicolumn{7}{|c|}{ Tag Muons in Muon Data } \\
Projective Cuts \\
\hline Type & $\begin{array}{c}p_{\mathrm{t}} \\
(\mathrm{GeV} / \mathrm{c})\end{array}$ & $\#$ OS & $\#$ SS & $\epsilon(\%)$ & $\mathrm{D}(\%)$ & $\epsilon D^{2}(\%)$ \\
\hline \hline$C M U$ & $2.0-2.5$ & 309 & 250 & $0.676 \pm 0.029$ & $10.6 \pm 4.2$ & $0.008 \pm 0.006$ \\
$C M U$ & $2.5-3.0$ & 200 & 191 & $0.473 \pm 0.024$ & $2.3 \pm 5.1$ & $0.000 \pm 0.001$ \\
$C M U$ & $3.0-4.0$ & 91 & 80 & $0.207 \pm 0.016$ & $6.4 \pm 7.6$ & $0.001 \pm 0.002$ \\
$C M U$ & $4.0+$ & 228 & 136 & $0.440 \pm 0.023$ & $25.3 \pm 5.1$ & $0.028 \pm 0.011$ \\
\hline$C M P$ & $3.0-4.0$ & 25 & 30 & $0.067 \pm 0.009$ & $-9.1 \pm 13.4$ & $-0.001 \pm 0.002$ \\
$C M P$ & $4.0+$ & 89 & 59 & $0.179 \pm 0.015$ & $20.3 \pm 8.0$ & $0.007 \pm 0.006$ \\
\hline$C M U P$ & $3.0-4.0$ & 120 & 97 & $0.263 \pm 0.018$ & $10.6 \pm 6.8$ & $0.003 \pm 0.004$ \\
$C M U P$ & $4.0+$ & 455 & 251 & $0.854 \pm 0.032$ & $28.9 \pm 3.6$ & $0.071 \pm 0.018$ \\
\hline$C M X$ & $2.0-2.5$ & 80 & 67 & $0.178 \pm 0.015$ & $8.8 \pm 8.2$ & $0.001 \pm 0.003$ \\
$C M X$ & $2.5-3.0$ & 71 & 55 & $0.152 \pm 0.014$ & $12.7 \pm 8.8$ & $0.002 \pm 0.003$ \\
$C M X$ & $3.0-4.0$ & 82 & 64 & $0.177 \pm 0.015$ & $12.3 \pm 8.2$ & $0.003 \pm 0.004$ \\
$C M X$ & $4.0+$ & 225 & 116 & $0.413 \pm 0.022$ & $32.0 \pm 5.1$ & $0.042 \pm 0.014$ \\
\hline Sum : & & 1975 & 1396 & $4.078 \pm 0.070$ & - & $0.165 \pm 0.028$ \\
\hline
\end{tabular}

Table 7.9: Number of same- and opposite-sign projective muons not in the trigger jet, as a function of $p_{\mathrm{t}}$, in trigger muon data . Dilutions have not been corrected for background or mixing in the trigger jet.
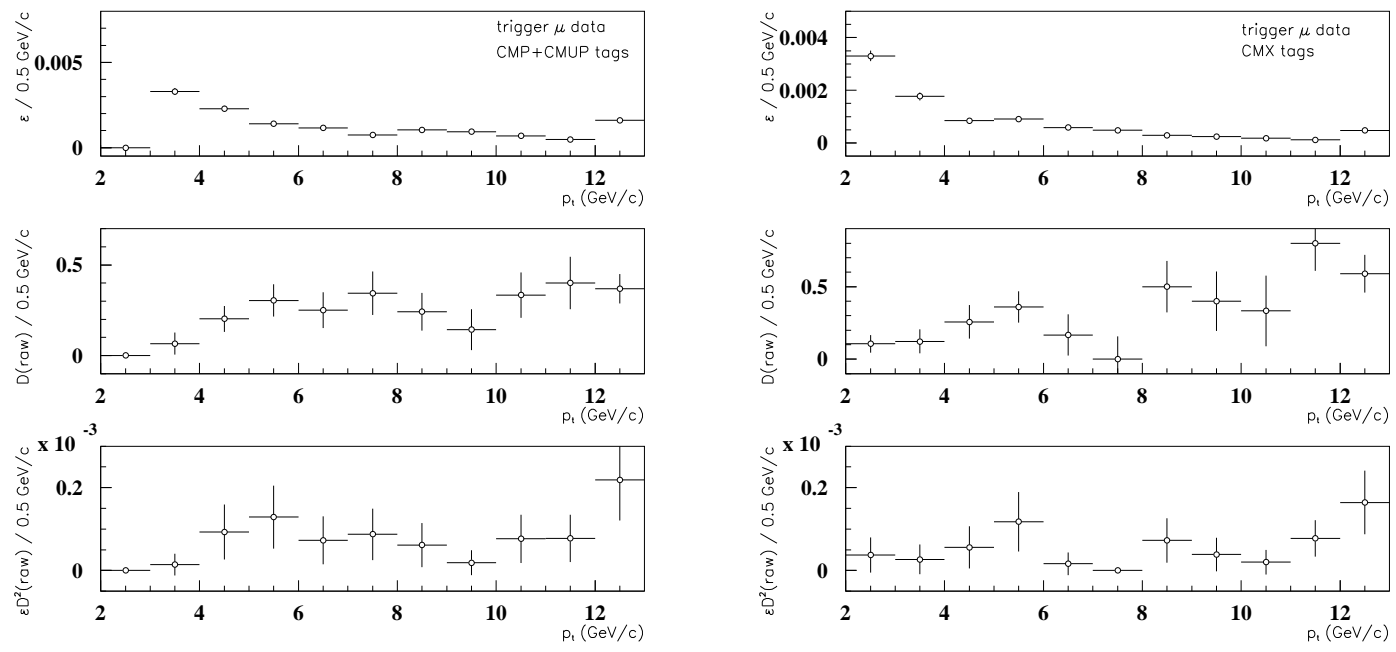

Figure 7.7: Top to bottom: efficiency, dilution, and $\epsilon D^{2}$ versus $p_{\mathrm{t}}$ for projective CMP and CMUP tags (left) and projective CMX tags (right) in trigger electron data. The rightmost bin is the overflow bin. Dilutions have not been corrected for background or mixing in the trigger jet. 


\begin{tabular}{|c|c|c|c|c|c|}
\hline \multicolumn{6}{|c|}{$\begin{array}{c}\text { Tag Muons in Electron Data } \\
\text { Projective Cuts }\end{array}$} \\
\hline Type & \#OS & \#SS & $\epsilon(\%)$ & $\mathrm{D}(\%)$ & $\epsilon D^{2}(\%)$ \\
\hline \multicolumn{6}{|c|}{ Integrated over $p_{t}^{\text {rel }}$} \\
\hline$C M U$ & 761 & 585 & $1.508 \pm 0.041$ & - & $0.042 \pm 0.013$ \\
\hline$C M P+C M U P$ & 665 & 396 & $1.189 \pm 0.036$ & - & $0.102 \pm 0.020$ \\
\hline$C M X$ & 410 & 297 & $0.792 \pm 0.030$ & - & $0.036 \pm 0.013$ \\
\hline Sum : & 1836 & 1278 & $3.489 \pm 0.063$ & - & $0.180 \pm 0.027$ \\
\hline \multicolumn{6}{|c|}{ Integrated over $p_{t}^{\text {comb }}$} \\
\hline$C M U$ & 761 & 585 & $1.508 \pm 0.041$ & - & $0.041 \pm 0.013$ \\
\hline$C M P+C M U P$ & 665 & 396 & $1.189 \pm 0.036$ & - & $0.095 \pm 0.020$ \\
\hline$C M X$ & 410 & 297 & $0.792 \pm 0.030$ & - & $0.038 \pm 0.012$ \\
\hline Sum : & 1836 & 1278 & $3.489 \pm 0.063$ & - & $0.174 \pm 0.027$ \\
\hline \multicolumn{6}{|c|}{ Muons without $p_{t}^{\text {re }}$} \\
\hline$C M U$ & 131 & 75 & $0.231 \pm 0.016$ & $27.2 \pm 6.7$ & $0.017 \pm 0.009$ \\
\hline$C M P+C M U P$ & 65 & 26 & $0.102 \pm 0.011$ & $42.9 \pm 9.5$ & $0.019 \pm 0.009$ \\
\hline$C M X$ & 75 & 52 & $0.142 \pm 0.013$ & $18.1 \pm 8.7$ & $0.005 \pm 0.005$ \\
\hline Sum : & 271 & 153 & $0.475 \pm 0.023$ & - & $0.041 \pm 0.013$ \\
\hline \multicolumn{5}{|l|}{ Total p pel } & $\overline{0.221 \pm 0.030}$ \\
\hline \multicolumn{5}{|l|}{ Total $p_{t}^{\text {comb }}$} & $0.215 \pm 0.030$ \\
\hline
\end{tabular}

Table 7.10: Summary of efficiencies and $\epsilon D^{2}$ versus $p_{\mathrm{t}}^{\text {rel }} \& p_{\mathrm{t}}^{\text {comb }}$ in trigger electron data. $\epsilon D^{2}$ for $p_{\mathrm{t}}^{\text {rel }}$ or $p_{\mathrm{t}}^{\text {comb }}$ binning methods is shown integrated over $p_{\mathrm{t}}^{\text {rel }}$ or $p_{\mathrm{t}}^{\text {comb }}$, respectively. $\epsilon D^{2}$ from tracks with no $p_{\mathrm{t}}^{\text {rel }}$ is shown separately. CMP and CMUP muons are combined, owing to low CMP statistics. Dilutions have not been corrected for background or mixing in the trigger jet. 


\begin{tabular}{|c|c|c|c|c|c|}
\hline \multicolumn{6}{|c|}{$\begin{array}{c}\text { Tag Muons in Muon Data } \\
\text { Projective Cuts }\end{array}$} \\
\hline Type & $\# \mathrm{OS}$ & \#SS & $\epsilon(\%)$ & $\mathrm{D}(\%)$ & $\epsilon D^{2}(\%)$ \\
\hline \multicolumn{6}{|c|}{ Integrated over $\mathrm{p}_{\mathrm{t}}^{\text {rel }}$} \\
\hline$C M U$ & 739 & 609 & $1.631 \pm 0.044$ & - & $0.037 \pm 0.013$ \\
\hline$C M P+C M U P$ & 642 & 413 & $1.276 \pm 0.039$ & - & $0.083 \pm 0.019$ \\
\hline$C M X$ & 403 & 274 & $0.819 \pm 0.031$ & - & $0.050 \pm 0.015$ \\
\hline Sum: & 1784 & 1296 & $3.726 \pm 0.067$ & - & $0.170 \pm 0.027$ \\
\hline \multicolumn{6}{|c|}{ Integrated over $p_{t}^{\text {comb }}$} \\
\hline$C M U$ & 739 & 609 & $1.631 \pm 0.044$ & - & $0.057 \pm 0.014$ \\
\hline$C M P+C M U P$ & 642 & 413 & $1.276 \pm 0.039$ & - & $0.080 \pm 0.019$ \\
\hline$C M X$ & 403 & 274 & $0.819 \pm 0.031$ & - & $0.060 \pm 0.015$ \\
\hline Sum : & 1784 & 1296 & $3.726 \pm 0.067$ & - & $0.197 \pm 0.028$ \\
\hline \multicolumn{6}{|c|}{ Muons without $p_{t}^{\text {rel }}$} \\
\hline$C M U$ & 89 & 48 & $0.166 \pm 0.014$ & $29.9 \pm 8.2$ & $0.015 \pm 0.008$ \\
\hline$C M P+C M U P$ & 47 & 24 & $0.086 \pm 0.010$ & $32.4 \pm 11.2$ & $0.009 \pm 0.006$ \\
\hline$C M X$ & 55 & 28 & $0.100 \pm 0.011$ & $32.5 \pm 10.4$ & $0.011 \pm 0.007$ \\
\hline Sum : & 191 & 100 & $0.352 \pm 0.021$ & - & $0.035 \pm 0.012$ \\
\hline \multicolumn{5}{|l|}{ Total $\mathrm{p}_{\mathrm{t}}^{\text {rel }}$} & $0.205 \pm 0.030$ \\
\hline \multicolumn{5}{|l|}{ Total $p_{t}^{\text {comb }}$} & $0.232 \pm 0.030$ \\
\hline
\end{tabular}

Table 7.11: Summary of efficiencies and $\epsilon D^{2}$ versus $p_{\mathrm{t}}^{\text {rel }} \& p_{\mathrm{t}}^{\text {comb }}$ in trigger muon data. $\epsilon D^{2}$ for $p_{\mathrm{t}}^{\text {rel }}$ or $p_{\mathrm{t}}^{\text {comb }}$ binning methods is shown integrated over $p_{\mathrm{t}}^{\text {rel }}$ or $p_{\mathrm{t}}^{\text {comb }}$, respectively. $\epsilon D^{2}$ from tracks with no $p_{\mathrm{t}}^{\text {rel }}$ is shown separately. CMP and CMUP muons are combined, owing to low CMP statistics. Dilutions have not been corrected for background or mixing in the trigger jet. 

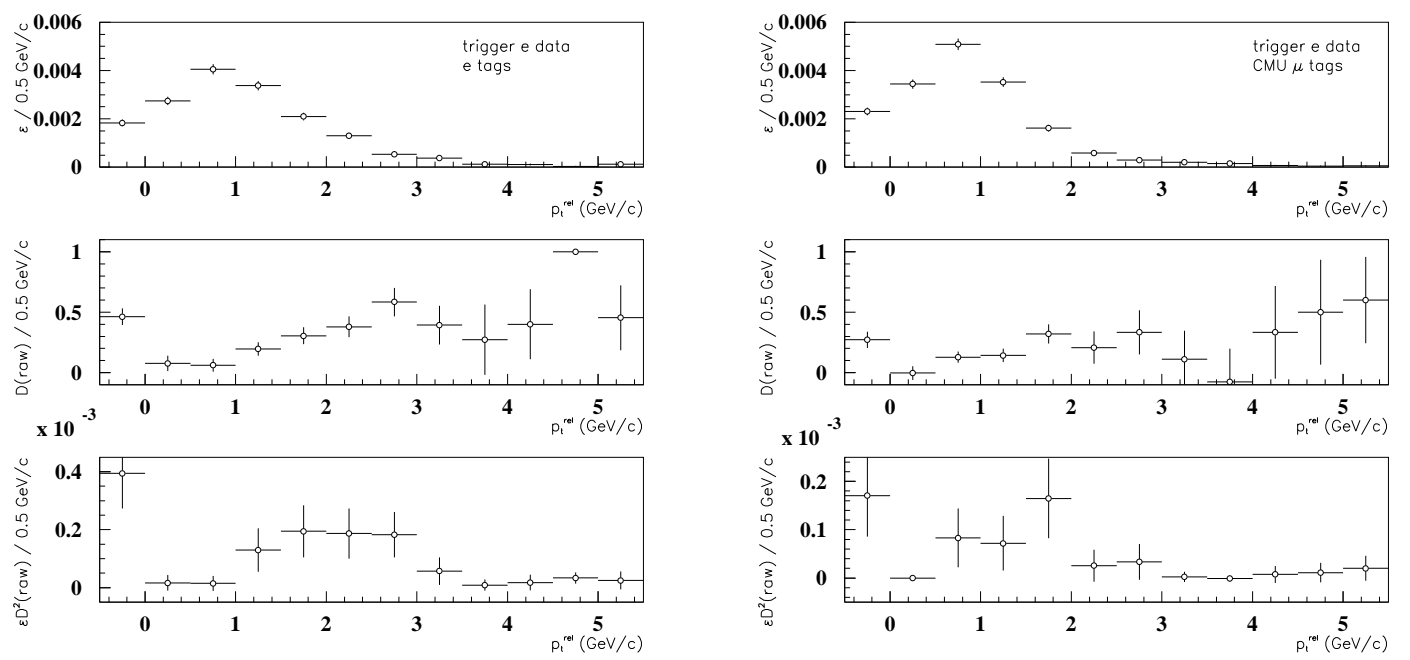

Figure 7.8: Top to bottom: efficiency, dilution, and $\epsilon D^{2}$ versus $p_{\mathrm{t}}^{\text {rel }}$ for soft electron tags (left) and projective CMU tags (right) in trigger electron data. The leftmost bin is for tags with no $p_{\mathrm{t}}^{\mathrm{rel}}$; the rightmost bin is the overflow bin. Dilutions have not been corrected for background or mixing in the trigger jet.
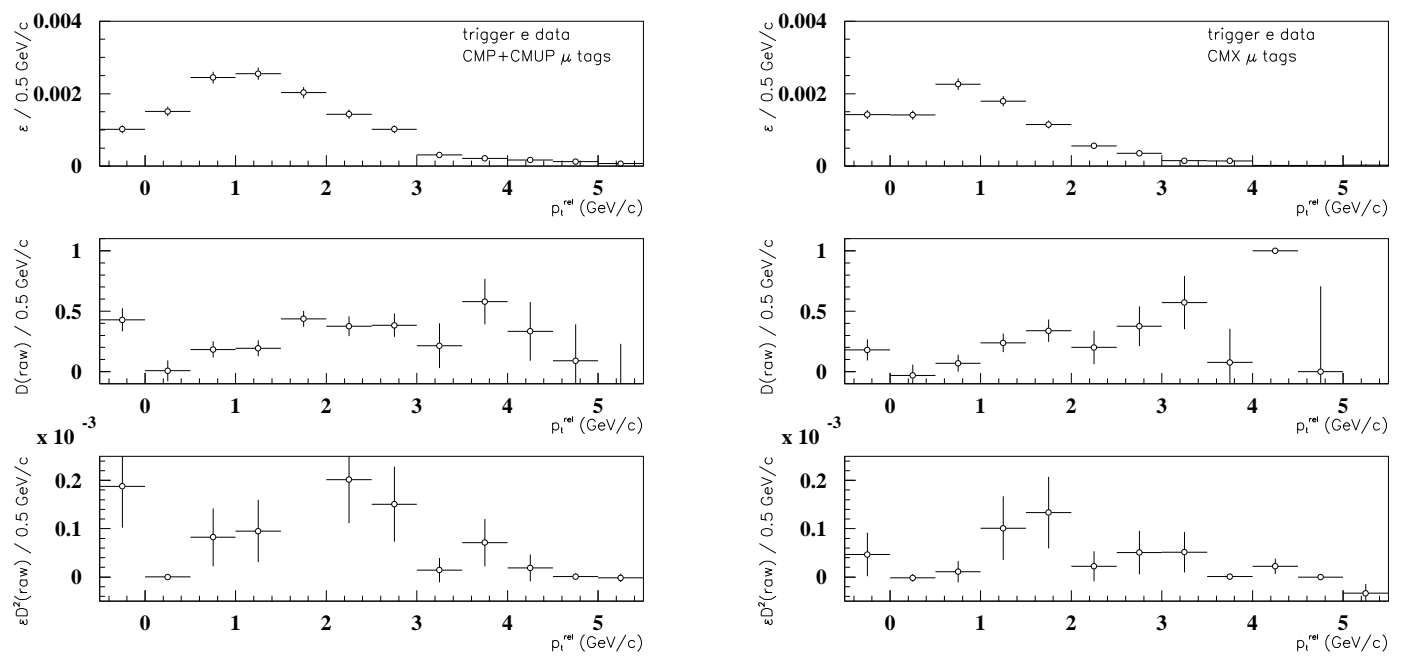

Figure 7.9: Top to bottom: efficiency, dilution, and $\epsilon D^{2}$ versus $p_{\mathrm{t}}^{\text {rel }}$ for projective CMP and CMUP tags (left) and projective CMX tags (right) in trigger electron data. The leftmost bin is for tags with no $p_{\mathrm{t}}^{\text {rel }}$; the rightmost bin is the overflow bin. Dilutions have not been corrected for background or mixing in the trigger jet. 

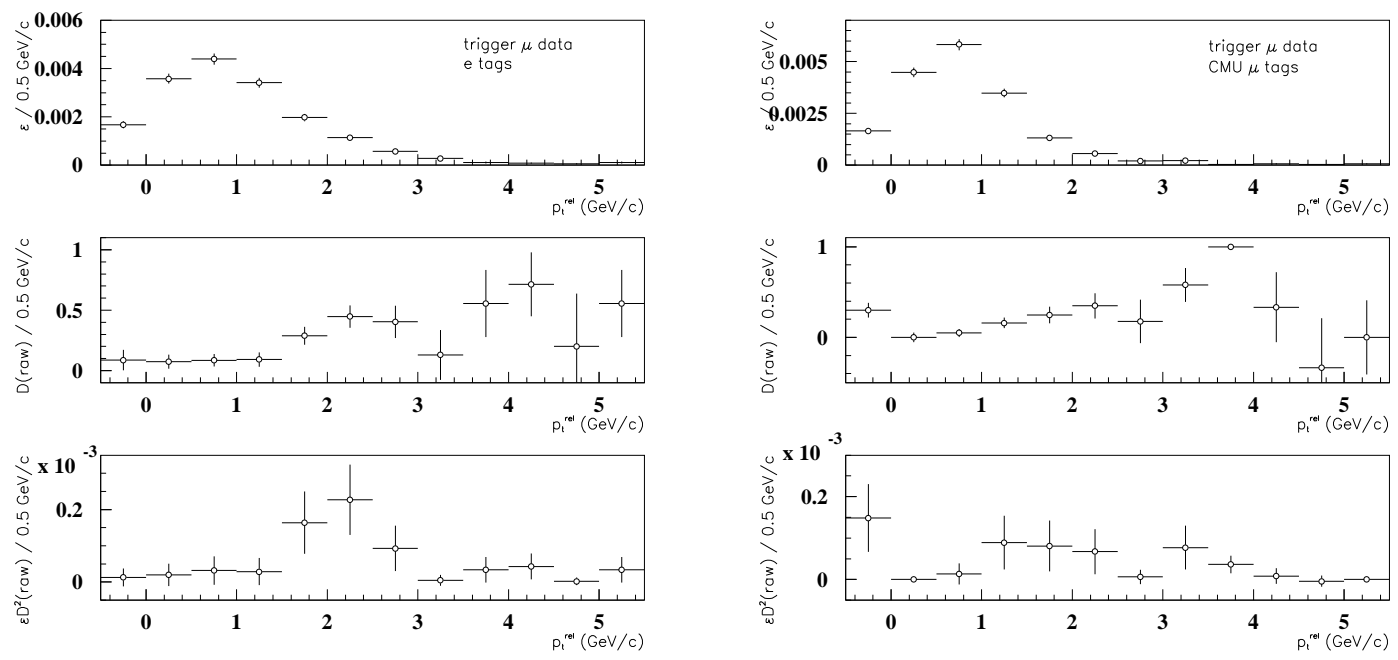

Figure 7.10: Top to bottom: efficiency, dilution, and $\epsilon D^{2}$ versus $p_{\mathrm{t}}^{\text {rel }}$ for soft electron tags (left) and projective CMU tags (right) in trigger muon data. The leftmost bin is for tags with no $p_{\mathrm{t}}^{\mathrm{rel}}$; the rightmost bin is the overflow bin. Dilutions have not been corrected for background or mixing in the trigger jet.
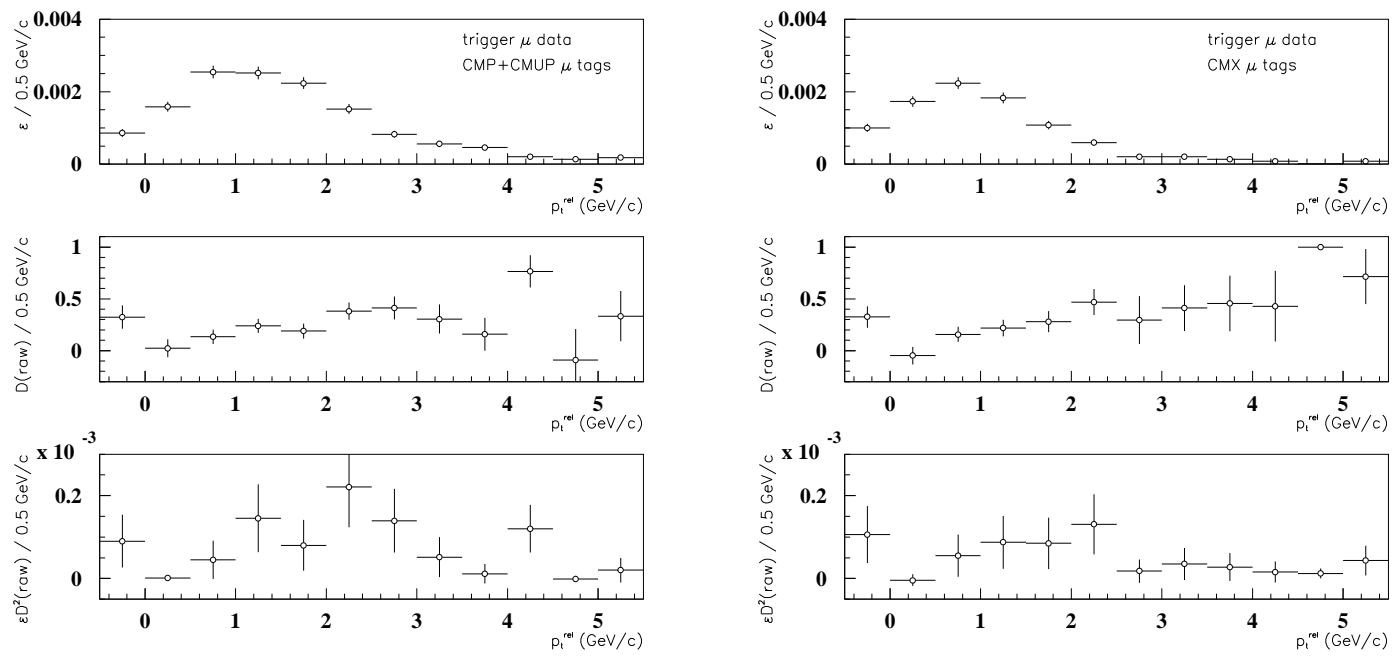

Figure 7.11: Top to bottom: efficiency, dilution, and $\epsilon D^{2}$ versus $p_{\mathrm{t}}^{\text {rel }}$ for projective CMP and CMUP tags (left) and projective CMX tags (right) in trigger electron data. The leftmost bin is for tags with no $p_{\mathrm{t}}^{\text {rel}}$; the rightmost bin is the overflow bin. Dilutions have not been corrected for background or mixing in the trigger jet. 

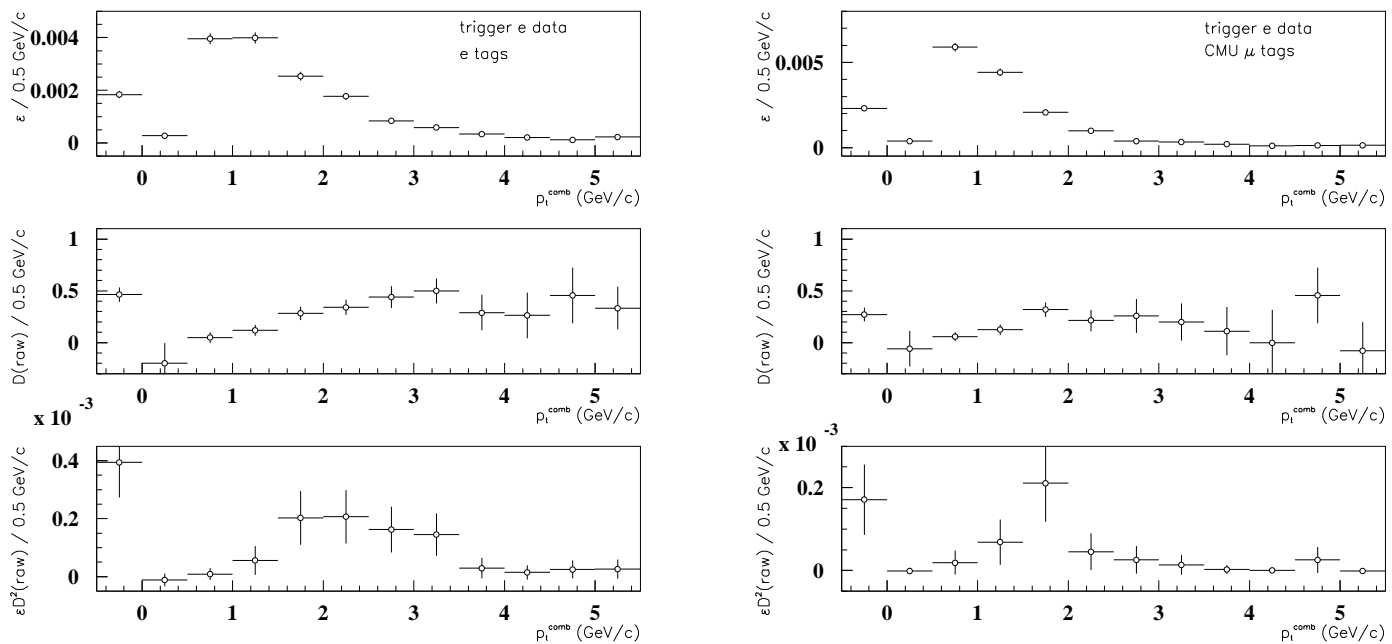

Figure 7.12: Top to bottom: efficiency, dilution, and $\epsilon D^{2}$ versus $p_{\mathrm{t}}^{\text {comb }}$ for soft electron tags (left) and projective CMU tags (right) in trigger electron data. The leftmost bin is for tags with no $p_{\mathrm{t}}^{\mathrm{comb}}$; the rightmost bin is the overflow bin. Dilutions have not been corrected for background or mixing in the trigger jet.
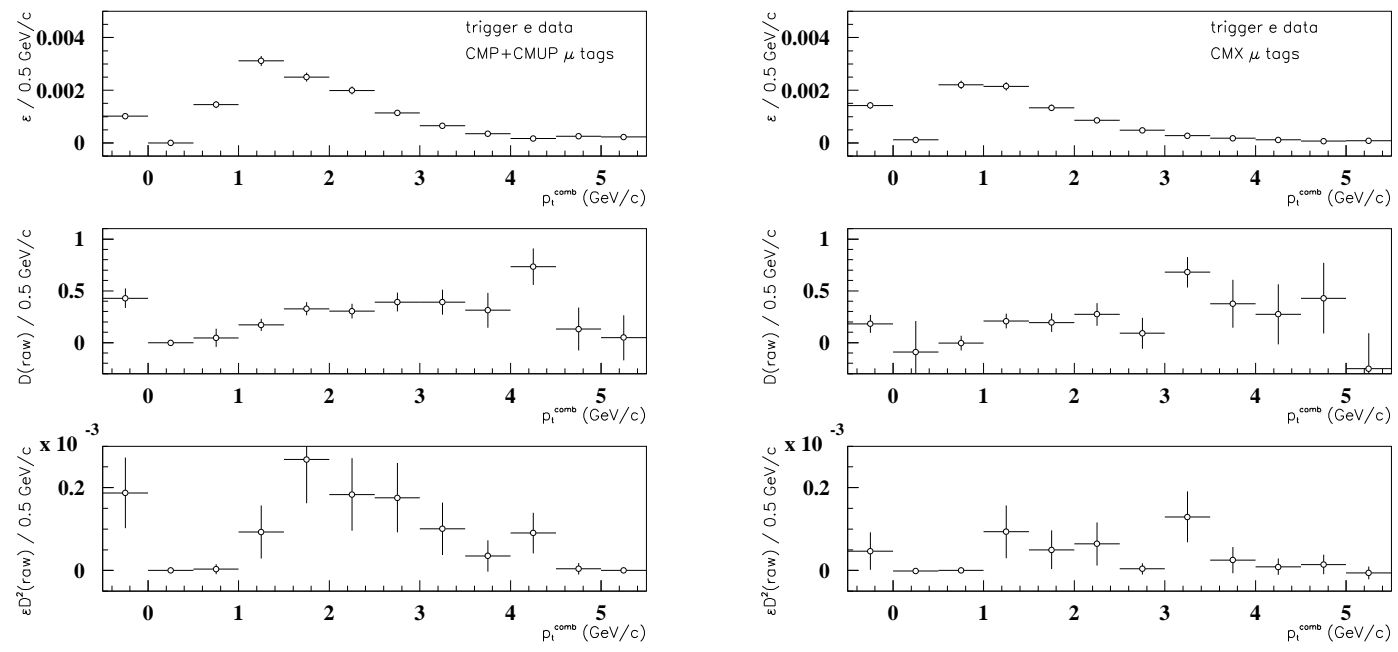

Figure 7.13: Top to bottom: efficiency, dilution, and $\epsilon D^{2}$ versus $p_{\mathrm{t}}^{\text {comb }}$ for projective CMP and CMUP tags (left) and projective CMX tags (right) in trigger electron data. The leftmost bin is for tags with no $p_{\mathrm{t}}^{\text {comb }}$; the rightmost bin is the overflow bin. Dilutions have not been corrected for background or mixing in the trigger jet. 

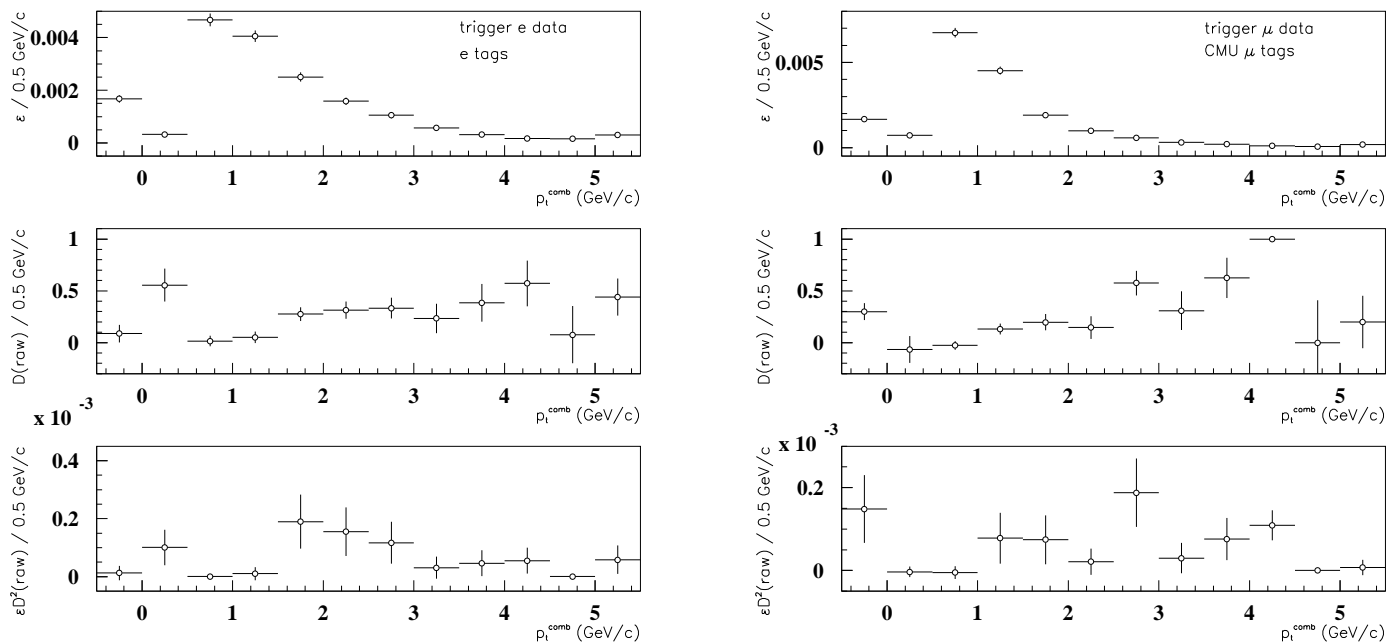

Figure 7.14: Top to bottom: efficiency, dilution, and $\epsilon D^{2}$ versus $p_{\mathrm{t}}^{\text {comb }}$ for soft electron tags (left) and projective CMU tags (right) in trigger muon data. The leftmost bin is for tags with no $p_{\mathrm{t}}^{\text {comb; }}$ the rightmost bin is the overflow bin. Dilutions have not been corrected for background or mixing in the trigger jet.
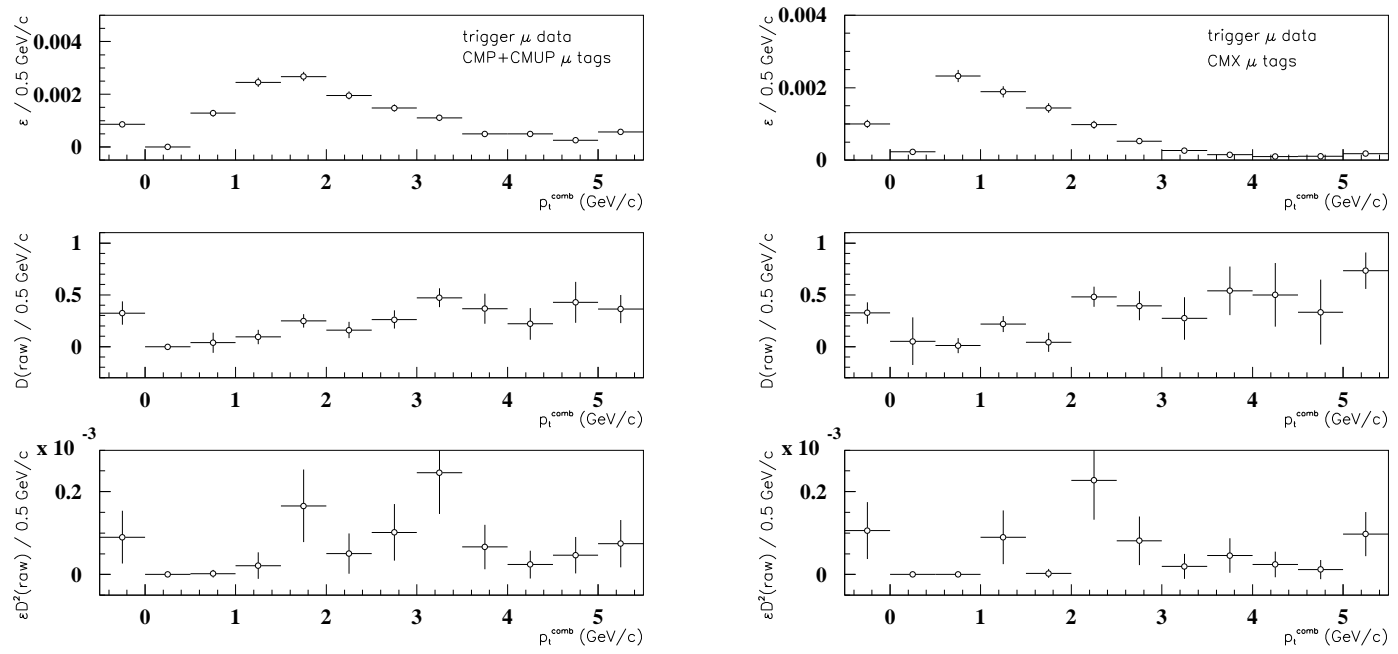

Figure 7.15: Top to bottom: efficiency, dilution, and $\epsilon D^{2}$ versus $p_{\mathrm{t}}^{\text {comb }}$ for projective CMP and CMUP tags (left) and projective CMX tags (right) in trigger electron data. The leftmost bin is for tags with no $p_{\mathrm{t}}^{\text {comb }}$; the rightmost bin is the overflow bin. Dilutions have not been corrected for background or mixing in the trigger jet. 
After a comparison of the soft electron and muon $\sum \epsilon D^{2}, p_{\mathrm{t}}^{\text {rel }}$ was selected as the kinematic variable to parametrize the dilution. It is clearly superior to $p_{\mathrm{t}}$, and though the soft muon total $\epsilon D^{2}$ is slightly higher when integrated over bins of $p_{\mathrm{t}}^{\text {comb }}$, the opposite is true for soft electrons. Given no clear distinction between the two, $p_{\mathrm{t}}^{\text {rel }}$ seems a more natural quantity, and suitably represents the dilution. It bears repeating that the SLT tags with no $p_{\mathrm{t}}^{\text {rel }}$ are handled separately; the raw dilution provided to the fit for $\Delta m_{d}$ is taken directly from Tables 7.6-7.7 and 7.10-7.11 for these tags.

Figures 7.16-7.18 again show the raw dilution versus $p_{\mathrm{t}}^{\mathrm{rel}}$, for the different SLT tag types. The function

$$
A\left[1-e^{-\left(p_{\mathrm{t}}^{\mathrm{rel}}-B\right)}\right]
$$

is used to parametrize the distribution of dilution with $p_{\mathrm{t}}^{\mathrm{rel}}$. $B$ describes the shape of the distribution, $A$ the normalization. Fits to the individual plots in Figures 7.167.18 are not particularly sensitive to the value of $B$, returning values in the range 0.22-0.33 with typical uncertainties of 0.11 . As the statistics are limited, a fit to all flavor-tags combined is used, returning a value for $B$ of 0.29 . $B$ is then fixed at this value, thus determining the shape of the dilution versus $p_{\mathrm{t}}^{\text {rel }}$. Fits for $A$ to the individual plots give the normalization; they yield

$\begin{array}{lcc}\text { Tag Type } & \text { Trigger e Data } & \text { Trigger } \mu \text { Data } \\ \text { Electron } & 0.39 \pm 0.04 & 0.35 \pm 0.05 \\ \text { All } \mu & 0.36 \pm 0.03 & 0.35 \pm 0.03 \\ \text { CMU } & 0.30 \pm 0.05 & 0.28 \pm 0.05 \\ \text { CMP + CMUP } & 0.43 \pm 0.05 & 0.37 \pm 0.04 \\ \text { CMX } & 0.36 \pm 0.06 & 0.43 \pm 0.06\end{array}$

The $\chi^{2}$ returned by the fits are also indicated in Figures 7.16-7.18. Every fit has an excellent $\chi^{2}$. 
Though the raw dilutions should vary based upon the different composition of the two samples, that they are near each other provides a nice consistency check. So, too, is the fact that the same shape so well describes the distributions for the different tags.
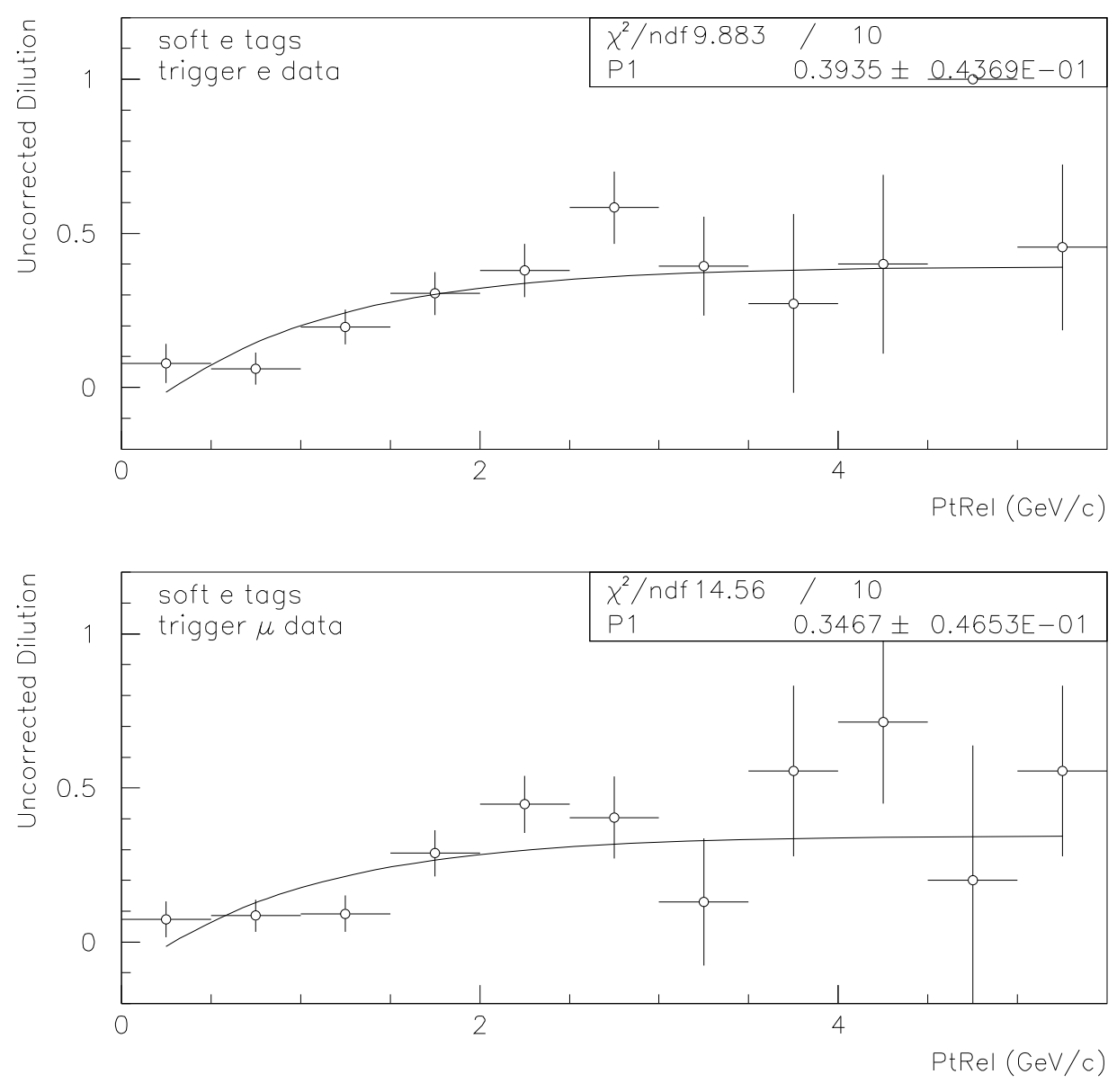

Figure 7.16: Raw dilution of soft electron tags as a function of $p_{\mathrm{t}}^{\text {rel }}$, for trigger electron data (top) and trigger muon data (bottom). The rightmost bin is the overflow bin; tags with no $p_{\mathrm{t}}^{\text {rel }}$ are not shown. The superimposed curves are $A\left[1-e^{-\left(p_{\mathrm{t}}^{\text {rel }}-0.290\right)}\right]$; a fit to $A$ is indicated on each plot. 

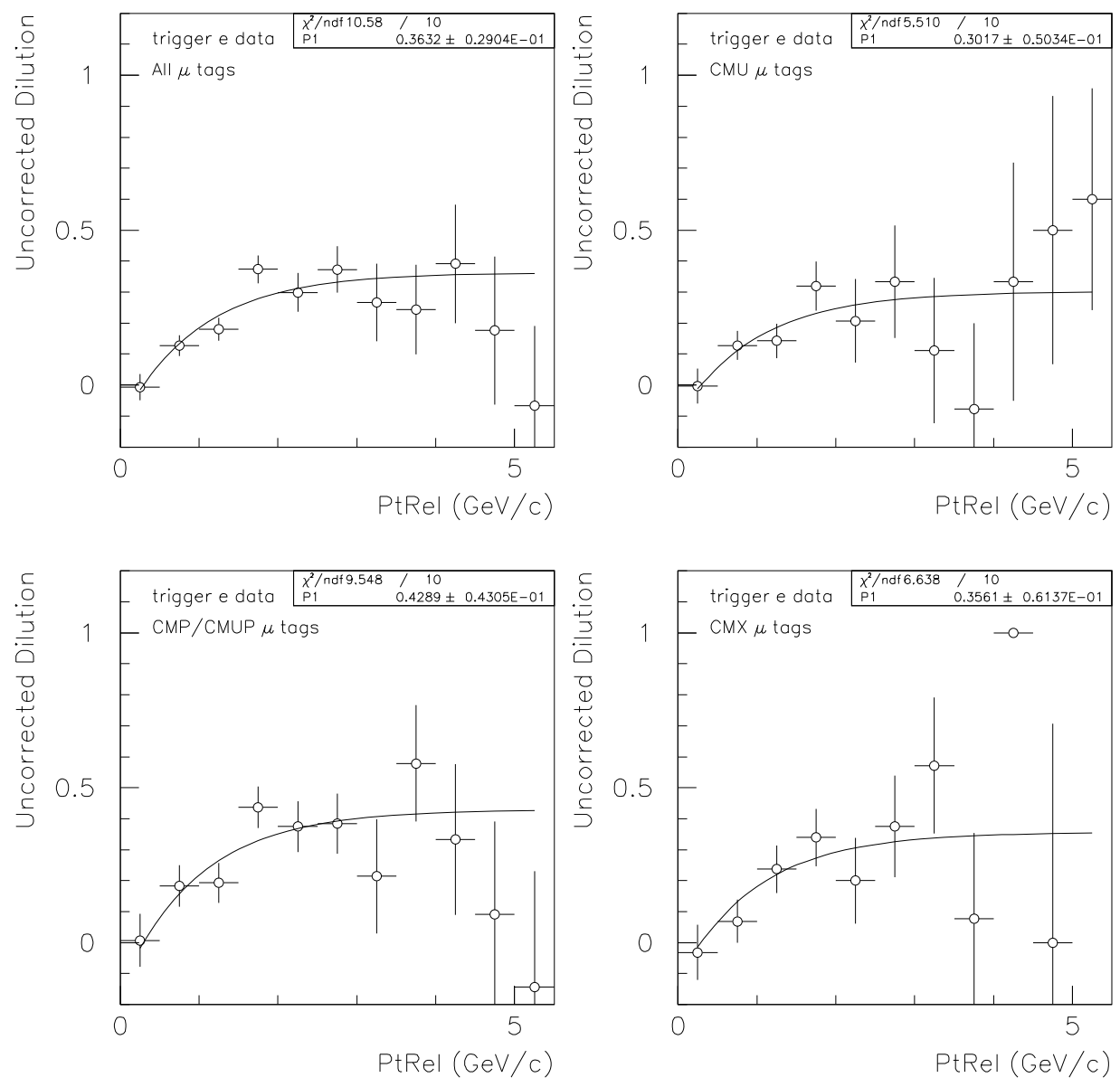

Figure 7.17: Raw dilution as a function of $p_{\mathrm{t}}^{\text {rel }}$ in trigger electron data, for all muon tags combined (upper left), CMU tags (upper right), CMP and CMUP tags (lower left), and CMX tags (lower right). The rightmost bin is the overflow bin; tags with no $p_{\mathrm{t}}^{\text {rel }}$ are not shown. The superimposed curves are $A\left[1-e^{-\left(p_{\mathrm{t}}^{\mathrm{rel}}-0.290\right)}\right]$; a fit to $A$ is indicated on each plot. 

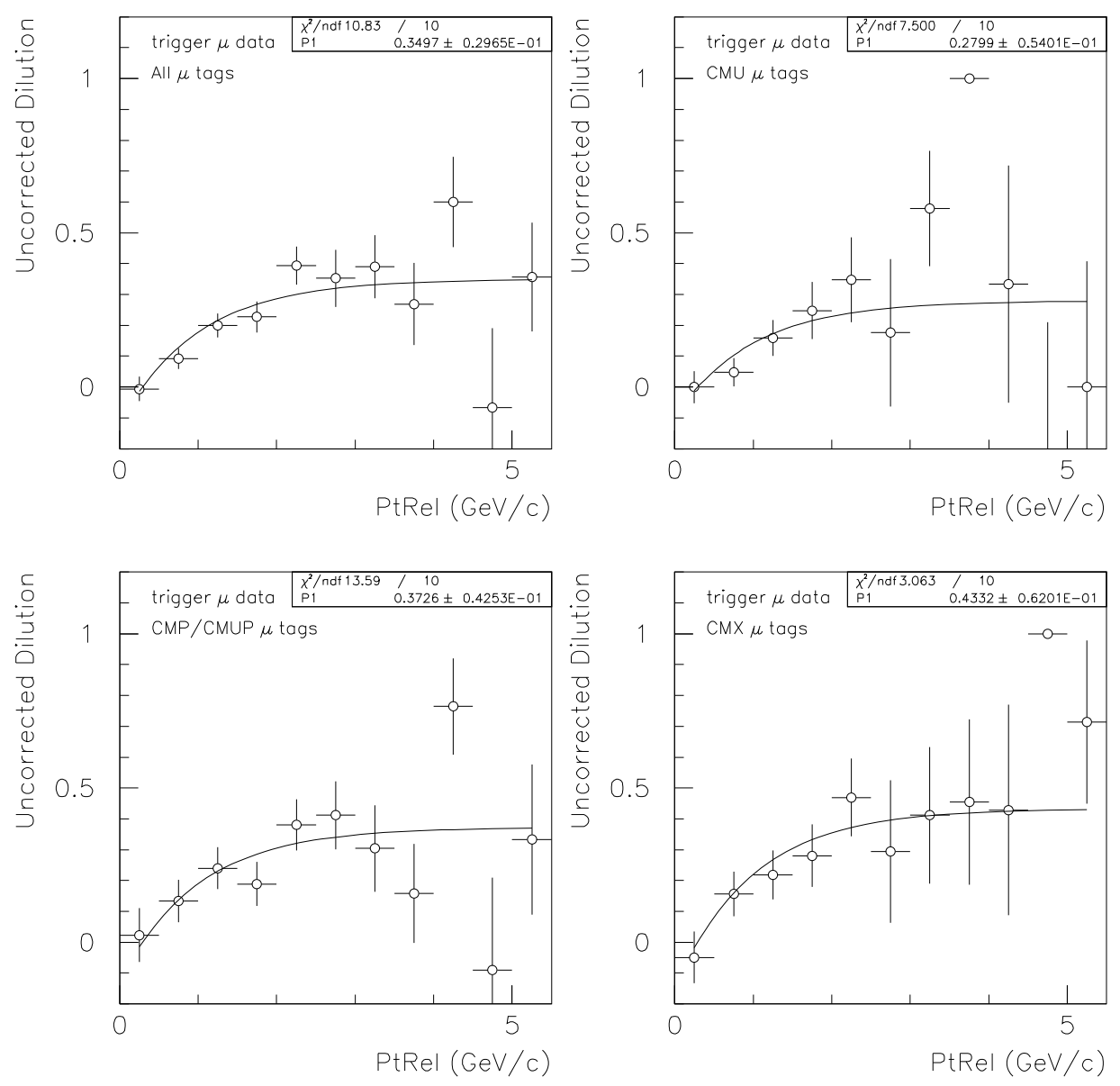

Figure 7.18: Raw dilution as a function of $p_{\mathrm{t}}^{\text {rel }}$ in trigger muon data, for all muon tags combined (upper left), CMU tags (upper right), CMP and CMUP tags (lower left), and CMX tags (lower right). The rightmost bin is the overflow bin; tags with no $p_{\mathrm{t}}^{\text {rel }}$ are not shown. The superimposed curves are $A\left[1-e^{-\left(p_{\mathrm{t}}^{\mathrm{rel}}-0.290\right)}\right]$; a fit to $A$ is indicated on each plot. 


\subsubsection{SLT Systematic Checks}

A number of checks were performed to assure that the soft lepton taggers are not subject to any systematic effects which could bias a measurement of $\Delta m_{d}$. First and foremost is if the taggers preferentially select positively-charged tracks over negatively-charged ones, or vice-versa, resulting in a charge asymmetry.

Antiprotons can easily fake an electron signature by annihilating with protons in the material of the solenoid, and create a cluster of electromagnetic energy indistinguishable from that produced by an electron. While the $d E / d x$ requirement serves to remove much of this background, it is less effective at higher particle momenta. Both protons and antiprotons are readily available from the accelerator beams, but since protons do not similarly annihilate in the detector material, this source of background could yield an excess of negatively-charged soft electron tags.

Fake muons from hadronic punchthrough are often charged kaons, which penetrate much further through matter than pions. Just as protons and antiprotons interact differently in matter, so do the charged kaons. $K^{+}$(composed of $\left.u \bar{s}\right)$ penetrate even further than $K^{-}$(composed of $\bar{u} s$ ) as the $\bar{u}$ antiquark in $K^{-}$can easily find an annihilation partner in the $u$ quark-rich protons and neutrons of the detector. (Were the CDF detector constructed of antimatter, the asymmetries would be reversed.)

Tables 7.12 and 7.13 show the number of lepton tags in the samples as a function of trigger lepton charge, tag lepton charge, and tag lepton $p_{\mathrm{t}}$.

While there is some statistical variation, there is no evidence for a systematic tag lepton charge asymmetry, except in the case of low- $p_{\mathrm{t}} \mathrm{CMU}$ tags. The CMP information for projective muons below $3 \mathrm{GeV} / \mathrm{c}$ is ignored; these CMU muons are expected to be the worst quality. Despite (rather, probably because of) the charge asymmetry, the dilution of the low- $p_{\mathrm{t}} \mathrm{CMU}$ tags is small (see Figures 7.4 and 7.6) 
and will not contribute significantly to the fit for $\Delta m_{d}$. This charge asymmetry can be safely ignored.

Considering trigger leptons, there is an obvious excess of positively-charged trigger muons (and no evidence for a charge asymmetry for trigger electrons). This is borne out by counting the number of positive and negative trigger leptons in the samples as a whole; there are 44,392 $\mathrm{e}^{+}$and 44,870 $\mathrm{e}^{-}$, and 42,454 $\mu^{+}$and 40,202 $\mu^{-}$, in their respective datasets. The trigger muon asymmetry, due to the previouslymentioned charge asymmetry for fakes, will be accounted for in the fit for $\Delta m_{d}$ by the fake lepton component of the sample composition.

Another possible systematic effect could be a dependence on the run number (which is, of course, a monotonically increasing function of the date the data were taken). Changes to the trigger hardware or software, or even damage to the detector, could produce such an effect. The efficiency and type-averaged dilution of the soft lepton tags versus run number is shown in Figures 7.19 and 7.20. Much more likely than overlooked detector damage is an instantaneous luminosity dependence. The average luminosity is closely correlated with run number; the Tevatron was operated at record luminosity at the end of Run 1B, balanced against its unexciting performance at the very beginning, when a quadrupole magnet was found to be misaligned. It is possible that the run-dependent figures mask a bias, as they do average over instantaneous luminosity. To assure no bias, Figures 7.21 and 7.22 explicitly show the same efficiencies and dilutions versus instantaneous luminosity. Within the available statistics, there is no convincing run or luminosity dependence.

One of the key responses which prevented the DAQ system from being completely overwhelmed by record luminosities was the adoption of dynamic trigger prescaling. As many of the single lepton triggers at Level 3 have no specific Level 2 requirement, a question was raised as to how many of the soft lepton tagged events 


\begin{tabular}{|c|r|r|r|r||r|r||r|r|}
\hline \multicolumn{7}{|c|}{$\begin{array}{c}\text { Soft Electron Tagger } \\
\text { Charge Asymmetry }\end{array}$} \\
\hline$p_{\mathrm{t}}$ range & $\ell^{+} \mu^{-}$ & $\ell^{-} \mu^{+}$ & $\ell^{-} \mu^{-}$ & $\ell^{+} \mu^{+}$ & $\ell^{+}$ & $\ell^{-}$ & $\mathrm{e}^{+}$ & $\mathrm{e}^{-}$ \\
\hline \hline \multicolumn{8}{|c|}{ Trigger Electron Sample } \\
\hline $2.0 \leq p_{\mathrm{t}}<2.5$ & 80 & 97 & 79 & 62 & 142 & 176 & 159 & 159 \\
$2.5 \leq p_{\mathrm{t}}<3.0$ & 67 & 76 & 48 & 49 & 116 & 124 & 125 & 115 \\
$3.0 \leq p_{\mathrm{t}}<4.0$ & 80 & 84 & 63 & 59 & 139 & 147 & 143 & 143 \\
$4.0 \leq p_{\mathrm{t}}$ & 228 & 200 & 115 & 104 & 332 & 315 & 304 & 343 \\
\hline \hline \multicolumn{8}{|c|}{ Trigger Muon Sample } \\
\hline $2.0 \leq p_{\mathrm{t}}<2.5$ & 83 & 85 & 59 & 81 & 164 & 144 & 166 & 142 \\
$2.5 \leq p_{\mathrm{t}}<3.0$ & 67 & 71 & 47 & 61 & 128 & 118 & 132 & 114 \\
$3.0 \leq p_{\mathrm{t}}<4.0$ & 74 & 73 & 66 & 52 & 126 & 139 & 125 & 140 \\
$4.0 \leq p_{\mathrm{t}}$ & 184 & 190 & 123 & 120 & 304 & 313 & 310 & 307 \\
\hline
\end{tabular}

Table 7.12: Number of tag electrons as a function of trigger lepton type and charge, in bins of $\operatorname{tag} p_{\mathrm{t}}$.

\begin{tabular}{|c|c|c|c|c|c|c|c|c|}
\hline \multicolumn{9}{|c|}{ Projective Muon Charge Asymmetry } \\
\hline Muon Type & $\ell^{+} \mu^{-}$ & $\overline{\ell^{-}} \mu^{+}$ & $\ell^{-} \mu^{-}$ & $\ell^{+} \mu^{+}$ & $\ell^{+}$ & $\ell^{-}$ & $\mu^{+}$ & $\mu^{-}$ \\
\hline \multicolumn{9}{|c|}{ Tag $p_{\mathrm{t}}<4.0 \mathrm{GeV} / \mathrm{c}$} \\
\hline \multicolumn{9}{|c|}{ Trigger Electron Sample } \\
\hline CMU & 316 & 366 & 234 & 272 & 588 & 600 & 638 & 550 \\
\hline $\mathrm{CMP}+\mathrm{CMUP}$ & 119 & 120 & 74 & 74 & 193 & 194 & 194 & 193 \\
\hline CMX & 142 & 140 & 97 & 119 & 261 & 237 & 259 & 239 \\
\hline \multicolumn{9}{|c|}{ Trigger Muon Sample } \\
\hline CMU & 296 & 304 & 233 & 288 & 584 & 537 & 592 & 529 \\
\hline $\mathrm{CMP}+\mathrm{CMUP}$ & 83 & 62 & 56 & 71 & 154 & 118 & 133 & 139 \\
\hline CMX & 116 & 117 & 82 & 104 & 220 & 199 & 221 & 198 \\
\hline \multicolumn{9}{|c|}{ Tag $p_{\mathrm{t}} \geq 4.0 \mathrm{GeV} / \mathrm{c}$} \\
\hline \multicolumn{9}{|c|}{ Trigger Electron Sample } \\
\hline $\mathrm{CMU}$ & 106 & 104 & 83 & 71 & 177 & 187 & 175 & 189 \\
\hline $\mathrm{CMP}+\mathrm{CMUP}$ & 233 & 258 & 143 & 131 & 364 & 401 & 389 & 376 \\
\hline CMX & 108 & 95 & 62 & 71 & 179 & 157 & 166 & 170 \\
\hline \multicolumn{9}{|c|}{ Trigger Muon Sample } \\
\hline $\mathrm{CMU}$ & 127 & 101 & 60 & 76 & 203 & 161 & 177 & 187 \\
\hline $\mathrm{CMP}+\mathrm{CMUP}$ & 293 & 251 & 149 & 161 & 454 & 400 & 412 & 442 \\
\hline CMX & 105 & 120 & 50 & 66 & 171 & 170 & 186 & 155 \\
\hline
\end{tabular}

Table 7.13: Number of tag muons as a function of trigger lepton charge and tag muon type, with a division at tag muon $p_{\mathrm{t}}=4.0 \mathrm{GeV} / \mathrm{c}$. 

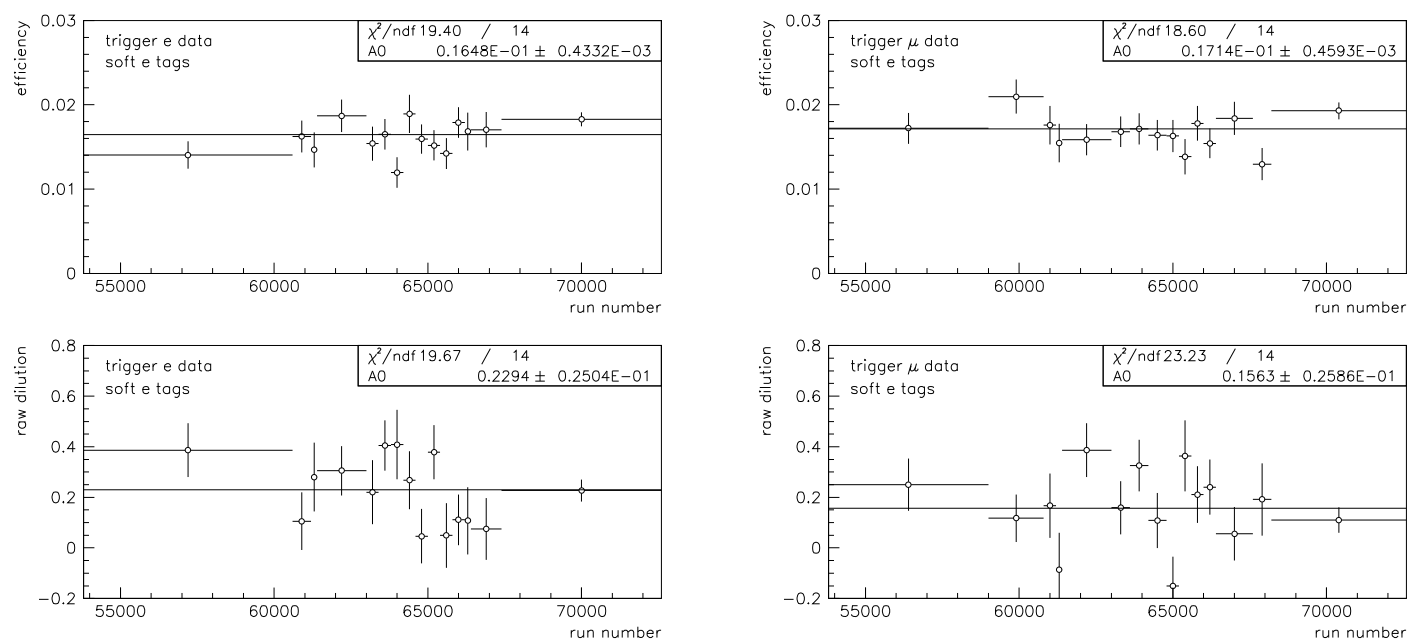

Figure 7.19: Tag electron efficiency and raw dilution versus run number in the trigger electron (left) and muon (right) samples.
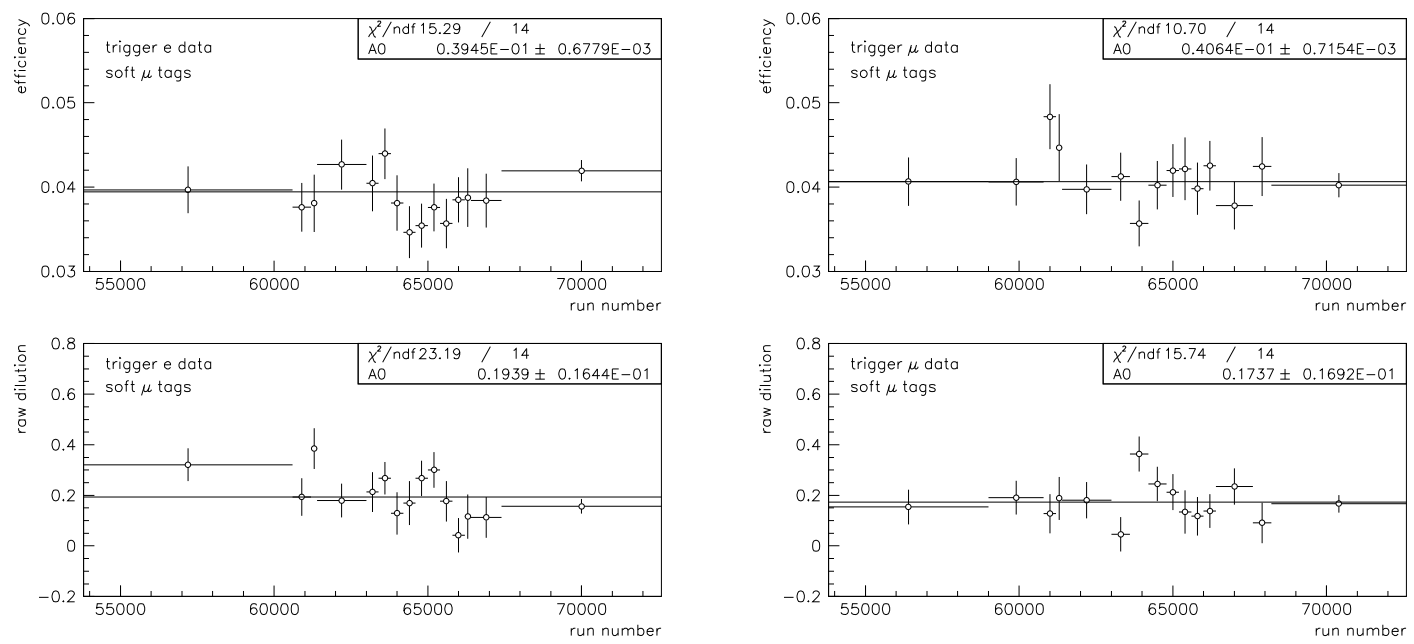

Figure 7.20: Tag muon efficiency and raw dilution versus run number in the trigger electron (left) and muon (right) samples. 

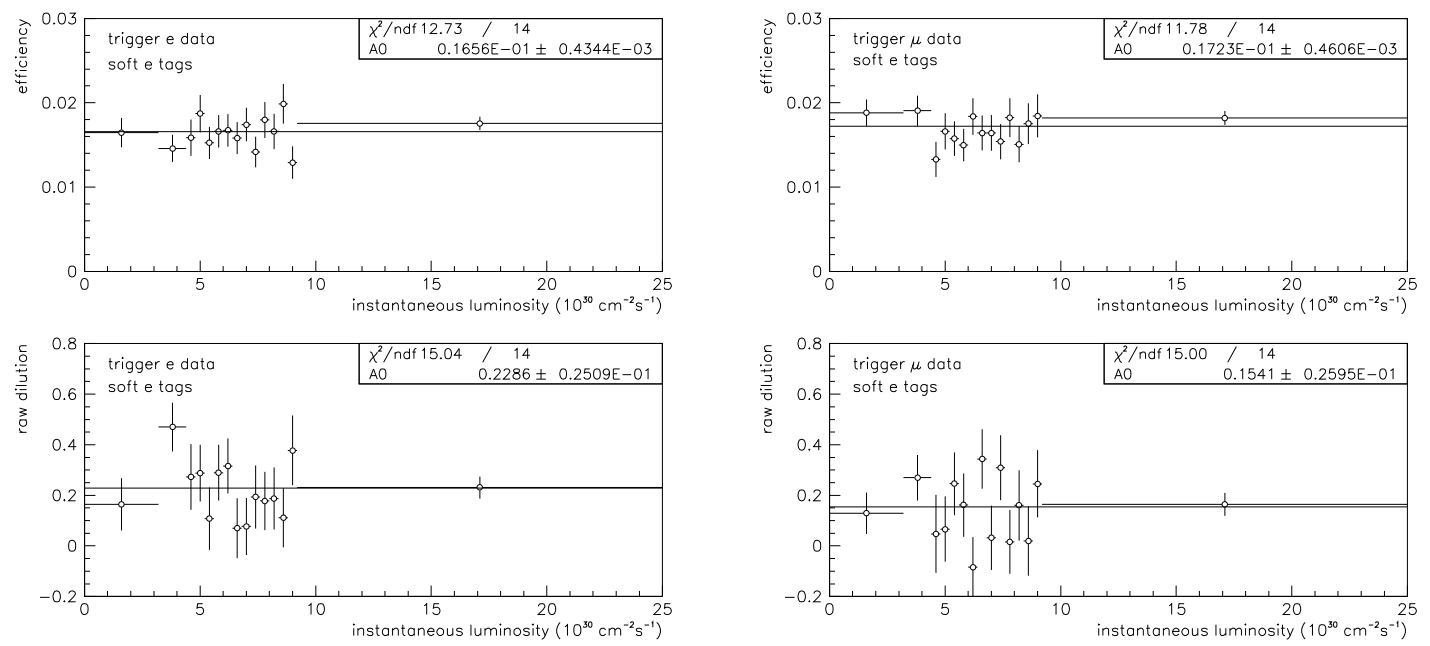

Figure 7.21: Tag electron efficiency and raw dilution versus instantaneous luminosity in the trigger electron (left) and muon (right) samples.
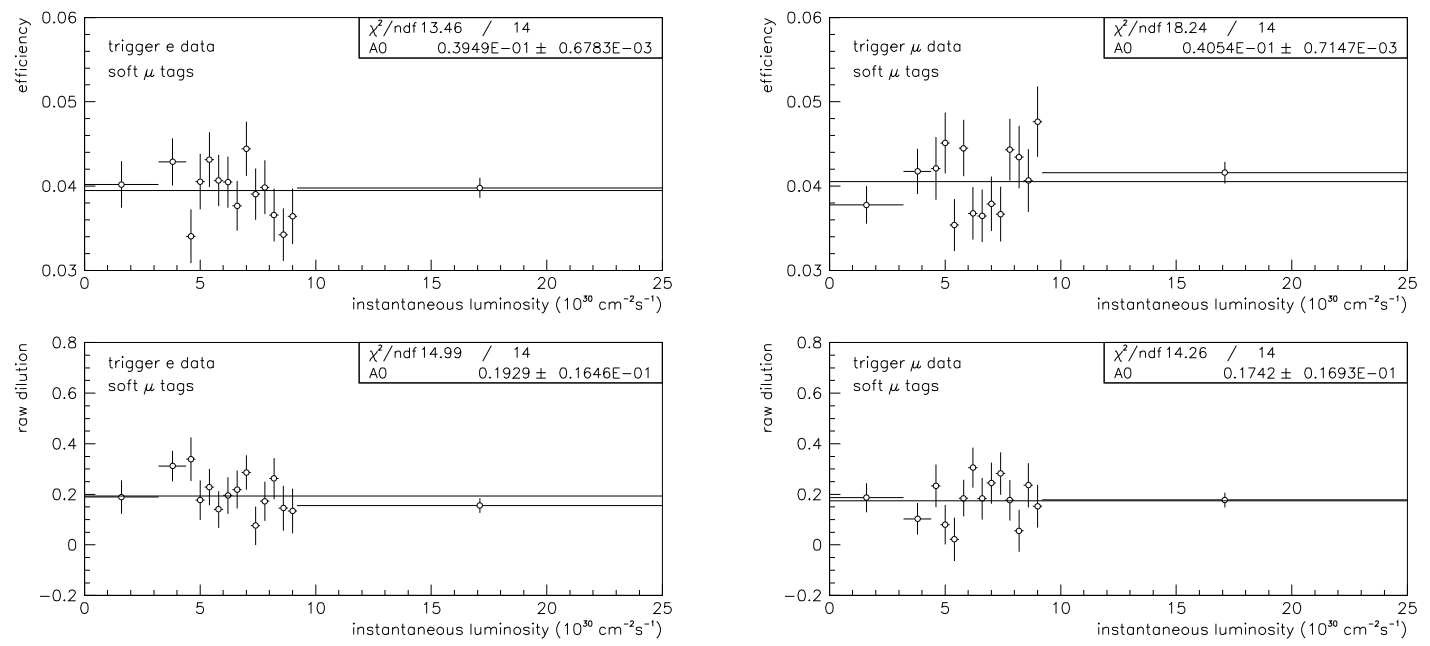

Figure 7.22: Tag muon efficiency and raw dilution versus instantaneous luminosity in the trigger electron (left) and muon (right) samples. 
in the samples came from single lepton Level 2 triggers as opposed to dilepton triggers, which, as a rule, were much less sharply prescaled under high-luminosity conditions. While a majority of the trigger leptons do have Level 2 single lepton triggers (see Sections 3.4.1 and 3.4.2), soft lepton tagging, which requires a second lepton in the event, might preferentially select Level 2 dilepton-triggered events, which may bias the SLT efficiency and dilution.

Applying a filter which determines whether the trigger electron or muon was the particular one responsible for a single lepton trigger at Level 2 reduces the electron sample to 82,550 events and the muon sample to 69,532 events. Tables $7.14-7.17$ show the parallel of the tagging results from Tables 7.6-7.9, making the Level 2 trigger requirement. A comparison of the two sets of tables shows that the presence of a single lepton trigger has a negligible effect on the SLT dilution, though the efficiency is somewhat lower. Since the dilution is unchanged, the conclusion is that the the trigger does not bias the flavor-tagging probabilities and, in fact, the dilepton triggers serve to increase the number of SLT-taggable events in the samples.

From the preceding information, one must conclude that there is no evidence to support a strong systematic bias adversely affecting the efficiency or dilution of SLT tagging from any of these factors. 


\begin{tabular}{|c|c|c|c|c|c|}
\hline \multicolumn{6}{|c|}{$\begin{array}{c}\text { Tag Electrons in Electron Data } \\
\text { Level } 2 \text { Single Electron Trigger Required }\end{array}$} \\
\hline $\begin{array}{c}p_{\mathrm{t}} \\
(\mathrm{GeV} / \mathrm{c})\end{array}$ & $\# \mathrm{OS}$ & $\# \mathrm{SS}$ & $\epsilon(\%)$ & $\mathrm{D}(\%)$ & $\epsilon D^{2}(\%)$ \\
\hline \multicolumn{6}{|c|}{ Versus $p_{t}$} \\
\hline $2.0-2.5$ & 163 & 127 & $0.351 \pm 0.021$ & $12.4 \pm 5.8$ & $0.005 \pm 0.005$ \\
\hline $2.5-3.0$ & 133 & 90 & $0.270 \pm 0.018$ & $19.3 \pm 6.6$ & $0.010 \pm 0.007$ \\
\hline $3.0-4.0$ & 147 & 113 & $0.315 \pm 0.020$ & $13.1 \pm 6.1$ & $0.005 \pm 0.005$ \\
\hline $4.0+$ & 362 & 182 & $0.659 \pm 0.028$ & $33.1 \pm 4.0$ & $0.072 \pm 0.018$ \\
\hline Sum : & 805 & 512 & $1.595 \pm 0.044$ & - & $0.092 \pm 0.021$ \\
\hline \multicolumn{6}{|c|}{ Integrated Over $p_{t}^{\text {rel }}$} \\
\hline$p_{\mathrm{t}}^{\mathrm{rel}}$ & 697 & 473 & $1.417 \pm 0.041$ & - & $0.089 \pm 0.019$ \\
\hline \multicolumn{6}{|c|}{ Integrated Over $p_{t}^{\text {comb }}$} \\
\hline$p_{\mathrm{t}}^{\text {comb }}$ & 697 & 473 & $1.417 \pm 0.041$ & - & $0.087 \pm 0.019$ \\
\hline \multicolumn{6}{|c|}{ Electrons without $p_{t}^{\text {rel }}$} \\
\hline no $p_{t}^{\text {rel }}$ & 108 & 39 & $0.178 \pm 0.015$ & $46.9 \pm 7.3$ & $0.039 \pm 0.013$ \\
\hline Total $\mathrm{p}_{\mathrm{t}}^{\text {rel }}$ & 805 & 512 & $1.595 \pm 0.044$ & - & $0.128 \pm 0.023$ \\
\hline Total $p_{t}^{\text {comb }}$ & 805 & 512 & $1.595 \pm 0.044$ & - & $0.126 \pm 0.023$ \\
\hline
\end{tabular}

Table 7.14: Tag electrons in trigger electron data, requiring that the trigger electron was responsible for a Level 2 single electron trigger. Dilutions have not been corrected for background or mixing in the trigger jet. 


\begin{tabular}{|c|c|c|c|c|c|}
\hline \multicolumn{6}{|c|}{$\begin{array}{l}\text { Tag Electrons in Muon Data } \\
\text { Level } 2 \text { Single Muon Trigger Required }\end{array}$} \\
\hline $\begin{array}{c}p_{\mathrm{t}} \\
(\mathrm{GeV} / \mathrm{c})\end{array}$ & $\# \mathrm{OS}$ & $\# \mathrm{SS}$ & $\epsilon(\%)$ & $\mathrm{D}(\%)$ & $\epsilon D^{2}(\%)$ \\
\hline \multicolumn{6}{|c|}{ Versus $p_{t}$} \\
\hline $2.0-2.5$ & 143 & 114 & $0.370 \pm 0.023$ & $11.3 \pm 6.2$ & $0.005 \pm 0.005$ \\
\hline $2.5-3.0$ & 113 & 85 & $0.285 \pm 0.020$ & $14.1 \pm 7.0$ & $0.006 \pm 0.006$ \\
\hline $3.0-4.0$ & 123 & 100 & $0.321 \pm 0.021$ & $10.3 \pm 6.7$ & $0.003 \pm 0.004$ \\
\hline $4.0+$ & 286 & 199 & $0.698 \pm 0.032$ & $17.9 \pm 4.5$ & $0.022 \pm 0.011$ \\
\hline Sum : & 665 & 498 & $1.673 \pm 0.049$ & - & $0.036 \pm 0.014$ \\
\hline \multicolumn{6}{|c|}{ Integrated Over $p_{t}^{\text {rel }}$} \\
\hline$p_{\mathrm{t}}^{\mathrm{rel}}$ & 598 & 443 & $1.497 \pm 0.046$ & - & $0.063 \pm 0.017$ \\
\hline \multicolumn{6}{|c|}{ Integrated Over $p_{t}^{\text {comb }}$} \\
\hline$p_{\mathrm{t}}^{\text {comb }}$ & 598 & 443 & $1.497 \pm 0.046$ & - & $0.059 \pm 0.018$ \\
\hline \multicolumn{6}{|c|}{ Electrons without $p_{t}^{\text {rel }}$} \\
\hline no $p_{t}^{\text {rel }}$ & 67 & 55 & $0.175 \pm 0.016$ & $9.8 \pm 9.0$ & $0.002 \pm 0.003$ \\
\hline Total $\mathrm{p}_{\mathrm{t}}^{\text {rel }}$ & 665 & 498 & $1.673 \pm 0.049$ & - & $0.065 \pm 0.017$ \\
\hline Total $p_{t}^{\text {comb }}$ & 665 & 498 & $1.673 \pm 0.049$ & - & $0.061 \pm 0.018$ \\
\hline
\end{tabular}

Table 7.15: Tag electrons in trigger muon data, requiring that the trigger muon was responsible for a Level 2 single muon trigger. Dilutions have not been corrected for background or mixing in the trigger jet. 


\begin{tabular}{|l|l|r|r|c|r|c|}
\hline \multicolumn{7}{|c|}{ Tag Muons in Electron Data } \\
\hline Type & $\begin{array}{c}p_{\mathrm{t}} \\
(\mathrm{GeV} / \mathrm{c})\end{array}$ & \#OS & \#SS & $\epsilon(\%)$ & $\mathrm{D}(\%)$ & $\epsilon D^{2}(\%)$ \\
\hline \hline \multicolumn{7}{|c|}{ Projective Cuts } \\
\hline$C M U$ & $2.0-2.5$ & 236 & 173 & $0.495 \pm 0.024$ & $15.4 \pm 4.9$ & $0.012 \pm 0.007$ \\
$C M U$ & $2.5-3.0$ & 198 & 127 & $0.394 \pm 0.022$ & $21.8 \pm 5.4$ & $0.019 \pm 0.009$ \\
$C M U$ & $3.0-4.0$ & 68 & 50 & $0.143 \pm 0.013$ & $15.3 \pm 9.1$ & $0.003 \pm 0.004$ \\
$C M U$ & $4.0+$ & 139 & 89 & $0.276 \pm 0.018$ & $21.9 \pm 6.5$ & $0.013 \pm 0.008$ \\
\hline$C M P$ & $3.0-4.0$ & 30 & 22 & $0.063 \pm 0.009$ & $15.4 \pm 13.7$ & $0.001 \pm 0.003$ \\
$C M P$ & $4.0+$ & 81 & 32 & $0.137 \pm 0.013$ & $43.4 \pm 8.5$ & $0.026 \pm 0.010$ \\
\hline$C M U P$ & $3.0-4.0$ & 113 & 77 & $0.230 \pm 0.017$ & $18.9 \pm 7.1$ & $0.008 \pm 0.006$ \\
$C M U P$ & $4.0+$ & 236 & 143 & $0.459 \pm 0.024$ & $24.5 \pm 5.0$ & $0.028 \pm 0.011$ \\
\hline$C M X$ & $2.0-2.5$ & 72 & 60 & $0.160 \pm 0.014$ & $9.1 \pm 8.7$ & $0.001 \pm 0.003$ \\
$C M X$ & $2.5-3.0$ & 78 & 46 & $0.150 \pm 0.013$ & $25.8 \pm 8.7$ & $0.010 \pm 0.007$ \\
$C M X$ & $3.0-4.0$ & 65 & 53 & $0.143 \pm 0.013$ & $10.2 \pm 9.2$ & $0.001 \pm 0.003$ \\
$C M X$ & $4.0+$ & 125 & 85 & $0.254 \pm 0.018$ & $19.0 \pm 6.8$ & $0.009 \pm 0.007$ \\
\hline Sum : & & 1441 & 957 & $2.905 \pm 0.059$ & - & $0.131 \pm 0.024$ \\
\hline
\end{tabular}

Table 7.16: Tag muons versus $p_{\mathrm{t}}$ in trigger electron data, requiring that the trigger electron was responsible for a Level 2 single electron trigger. Dilutions have not been corrected for background or mixing in the trigger jet. 


\begin{tabular}{|l|l|r|r|c|r|r|}
\hline \multicolumn{7}{|c|}{ Tag Muons in Muon Data } \\
\hline Type & $\begin{array}{c}p_{\mathrm{t}} \\
(\mathrm{GeV} / \mathrm{c})\end{array}$ & \#OS & $\#$ SS & $\epsilon(\%)$ & \multicolumn{1}{c|}{$\mathrm{D}(\%)$} & $\epsilon D^{2}(\%)$ \\
& & & & \\
\hline \hline \multicolumn{7}{|c|}{ Projective Cuts } \\
\hline$C M U$ & $2.0-2.5$ & 210 & 178 & $0.558 \pm 0.028$ & $8.2 \pm 5.1$ & $0.004 \pm 0.005$ \\
$C M U$ & $2.5-3.0$ & 137 & 139 & $0.397 \pm 0.024$ & $-0.7 \pm 6.0$ & $0.000 \pm 0.000$ \\
$C M U$ & $3.0-4.0$ & 71 & 58 & $0.186 \pm 0.016$ & $10.1 \pm 8.8$ & $0.002 \pm 0.003$ \\
$C M U$ & $4.0+$ & 158 & 97 & $0.367 \pm 0.023$ & $23.9 \pm 6.1$ & $0.021 \pm 0.011$ \\
\hline$C M P$ & $3.0-4.0$ & 18 & 24 & $0.060 \pm 0.009$ & $-14.3 \pm 15.3$ & $-0.001 \pm 0.003$ \\
$C M P$ & $4.0+$ & 68 & 47 & $0.165 \pm 0.015$ & $18.3 \pm 9.2$ & $0.006 \pm 0.006$ \\
\hline$C M U P$ & $3.0-4.0$ & 84 & 63 & $0.211 \pm 0.017$ & $14.3 \pm 8.2$ & $0.004 \pm 0.005$ \\
$C M U P$ & $4.0+$ & 268 & 138 & $0.584 \pm 0.029$ & $32.0 \pm 4.7$ & $0.060 \pm 0.018$ \\
\hline$C M X$ & $2.0-2.5$ & 69 & 57 & $0.181 \pm 0.016$ & $9.5 \pm 8.9$ & $0.002 \pm 0.003$ \\
$C M X$ & $2.5-3.0$ & 55 & 47 & $0.147 \pm 0.015$ & $7.8 \pm 9.9$ & $0.001 \pm 0.002$ \\
$C M X$ & $3.0-4.0$ & 63 & 52 & $0.165 \pm 0.015$ & $9.6 \pm 9.3$ & $0.002 \pm 0.003$ \\
$C M X$ & $4.0+$ & 167 & 89 & $0.368 \pm 0.023$ & $30.5 \pm 6.0$ & $0.034 \pm 0.014$ \\
\hline Sum : & & 1368 & 989 & $3.390 \pm 0.070$ & - & $0.135 \pm 0.027$ \\
\hline
\end{tabular}

Table 7.17: Tag muons versus $p_{\mathrm{t}}$ in trigger muon data, requiring that the trigger muon was responsible for a Level 2 single muon trigger. Dilutions have not been corrected for background or mixing in the trigger jet. 


\subsection{Jet Charge Flavor-Tagging}

The goal of jet charge flavor-tagging, just as for soft lepton tagging, is to identify the flavor of the second $b$ in an event. This is accomplished by selecting a likely candidate jet for the second $b$ and calculating the momentum-weighted charge average of the tracks within it. The jet charge $\left(Q_{j e t}\right)$ has been found [35] to be correlated with the charge of the quark which produced the jet ${ }^{3}$. One can think of a number of reasons why this should be so:

- In the case of fully-hadronic decay, the decay chain $b \rightarrow c \rightarrow s$ produces a $K$ with the same charge as its grandparent $\left(b \rightarrow K^{-}, \bar{b} \rightarrow K^{+}\right)$

- Even when this $K$ is neutral (as the $K$ from the decay of a neutral $B$ usually is), the leading fragmentation particle will have the same charge as the $b$ quark $\left(b \rightarrow b \bar{d} d \bar{u} u+\cdots \rightarrow \bar{B}_{d}^{0} \pi^{-} u+\cdots\right)$.

- The virtual $W$ from the weak decay of a $b$ quark carries away the charge of the $b$; whether it decays hadronically or leptonically, it will still have the correct charge correlation (recall that it is the leptonic $W$ decay which is exploited by the SLT tagger).

By adjusting how the momenta of the tracks in a jet are weighted, the jet charge algorithm can be tuned to be sensitive to these processes, producing the appropriate correlation between $Q_{j e t}$ and the $b$ flavor.

Jet charge tagging has been used to stunning success by the LEP experiments $[35,37,64]$ - the energetic $b$ jets from $Z^{0}$ decay are quite narrow, and the $e^{+} e^{-}$collider environment is particularly clean. Though CDF's $p \bar{p}$ environment

\footnotetext{
${ }^{3}$ The average $Q_{\text {jet }}$ for hard gluon jets is, of course, zero.
} 
makes jet charge tagging challenging, owing to lower- $p_{\mathrm{t}} b \bar{b}$ production and backgrounds from the underlying event, one cannot deny that it is effective even so, based upon the study that follows.

\subsubsection{Jet Selection and Definition of $Q_{\text {jet }}$}

The jet charge algorithm makes use of the TRKSJT jets made with a cone size of $\Delta R=0.8$. Only those jets with a $\sum p_{\mathrm{t}}>5.0 \mathrm{GeV} / \mathrm{c}$ and $\Delta \phi>90^{\circ}$ relative to the trigger lepton are considered. Of these, the one with the highest $\sum p_{\mathrm{t}}$ is taken to be the second $b$ jet. Because the angular cut selects jets in the opposite hemisphere from the trigger lepton, this jet is called the "opposite side jet". The efficiency for

finding such a jet is approximately $50 \%$; the $b \bar{b}$ Monte Carlo samples predict that this jet is the other $b$ jet approximately $75 \%$ of the time. The remaining $25 \%$ of the jets are most often gluon jets, and have no flavor correlation.

The jet charge $\left(Q_{j e t}\right)$ is defined by weighting the charges of the tracks in the jet by some power of their momenta projected along the jet axis:

$$
Q_{\text {jet }}=\frac{\sum_{i=1}^{n} q_{i} \cdot\left(\overrightarrow{p_{i}} \cdot \hat{a}\right)^{\kappa}}{\sum_{i=1}^{n}\left(\overrightarrow{p_{i}} \cdot \hat{a}\right)^{\kappa}}
$$

where the unit vector $\hat{a}$ is the jet axis and $\kappa$ adjusts the relative importance of each track's momentum. $\kappa=0$ weights all tracks equally; the limit of $\kappa \rightarrow \infty$ sets $Q_{\text {jet }}$ to the charge of the track with the largest $\overrightarrow{p_{i}} \cdot \hat{a} . \kappa=1$ is chosen for this analysis, as this value gives a fairly shallow maximum $\epsilon D^{2}$.

\subsubsection{Jet Charge Dilutions}

As the opposite side jet charge is correlated with the charge of its $b$ quark, just as is the charge of the soft lepton tags, it will be anti-correlated with the charge of the 
trigger lepton. Thus, $Q_{\ell} \cdot Q_{\text {jet }}$ should (on average) be negative for trigger $\ell^{+}$and positive for trigger $\ell^{-}$. The $Q_{\ell} \cdot Q_{j e t}$ distributions for the data and $b \bar{b}$ Monte Carlo samples are shown in Figure 7.23. Given an opposite side jet, if one takes $Q_{j e t}<0$ $(>0)$ to have tagged the jet as coming from a $b(\bar{b})$ quark, then the average dilution is

$$
\frac{N\left(Q_{\ell^{+}} Q_{j e t}<0\right)+N\left(Q_{\ell^{-}} Q_{j e t}>0\right)-N\left(Q_{\ell^{+}} Q_{j e t}>0\right)-N\left(Q_{\ell^{-}} Q_{j e t}<0\right)}{N\left(Q_{\ell^{+}} Q_{j e t}<0\right)+N\left(Q_{\ell^{-}} Q_{j e t}>0\right)+N\left(Q_{\ell^{+}} Q_{j e t}>0\right)+N\left(Q_{\ell^{-}} Q_{j e t}<0\right)}
$$

As an event-by-event dilution is desired, the dilution can be parametrized as a function of $\left|Q_{\text {jet }}\right|$. It may not be immediately apparent in Figure 7.23, but the absolute

difference $\left|N\left(Q_{\ell^{+}} Q_{\text {jet }}\right)-N\left(Q_{\ell^{-}} Q_{j e t}\right)\right|$ is larger for larger values of the absolute jet charge. Figures 7.24 and 7.25 show the dilution as defined in Equation 7.10 in bins of $\left|Q_{j e t}\right|$, as well as the number of jet charge-tagged events and $\epsilon D^{2}$ per bin, for the data and $b \bar{b}$ Monte Carlo samples, respectively. The dilution can be parametrized as a linear function of $\left|Q_{\text {jet }}\right|$; the fit parameter is the slope, which is also indicated in the figures.

\subsubsection{Jet Charge Corrections}

Just as a charge asymmetry can produce a systematic bias in the soft lepton flavortagging, so can it affect jet charge flavor-tagging. The source of the asymmetry, however, is different. The tracks which are used to compute the jet charge can have a $p_{\mathrm{t}}$ as low as $0.4 \mathrm{GeV} / \mathrm{c}$. For very low- $p_{\mathrm{t}}$ tracks, the CTC track-reconstruction efficiency is slightly worse for negatively-charged tracks. Calculating the charge asymmetry from the number of positive and negative tracks in opposite side jets

$$
A^{ \pm}=\frac{N^{+}-N^{-}}{N^{+}+N^{-}}
$$



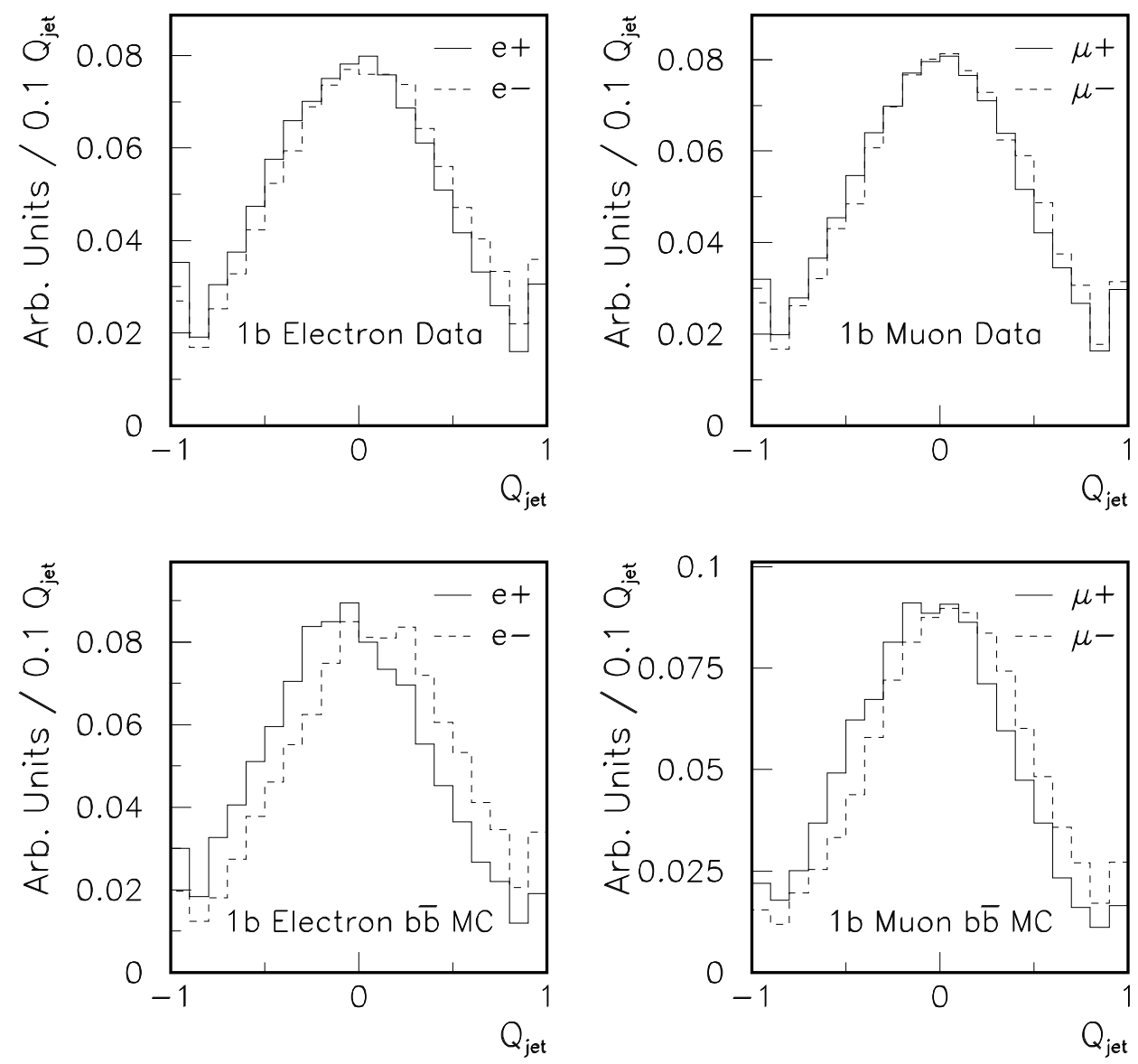

Figure 7.23: Jet charge distributions for the data and $b \bar{b}$ Monte Carlo samples. The distributions for positively-charged (solid) and negatively-charged (dashed) trigger leptons are shown separately. The "spikes" at $\left|Q_{j e t}\right|=1$ are from jets where all tracks have the same charge. All distributions are normalized to unit area. 
1b Electron Data
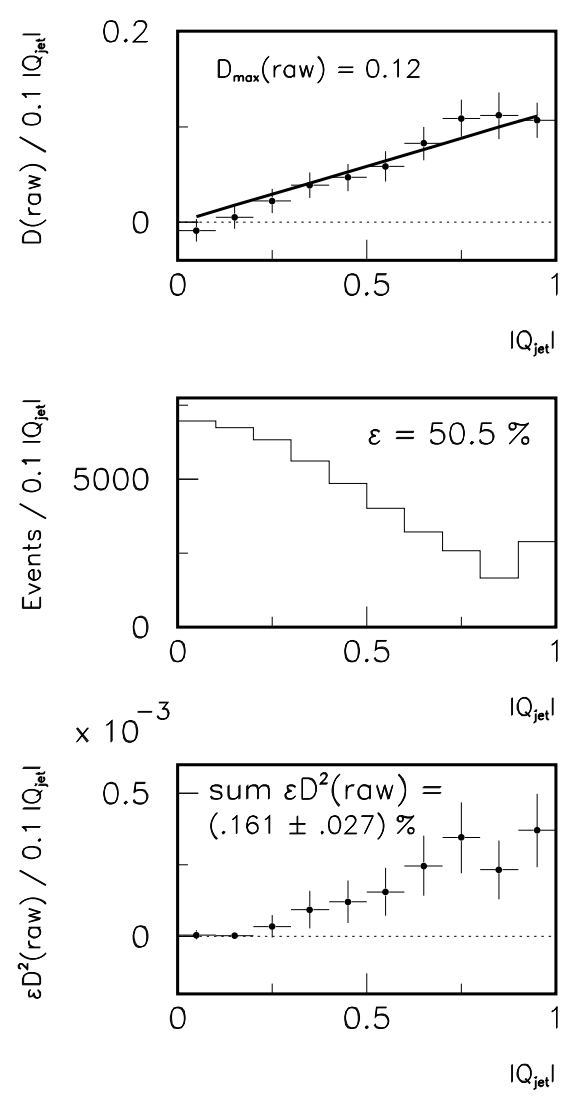

1b Muon Data
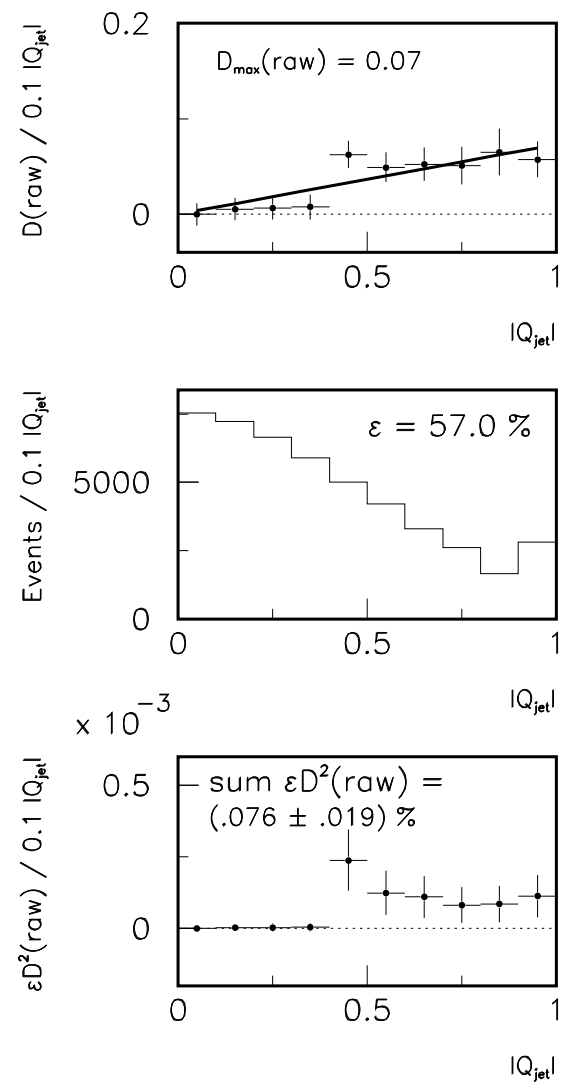

Figure 7.24: Jet charge flavor-tagging dilution, efficiency, and $\epsilon D^{2}$ in the data samples. Top left and right: $D($ raw $)$ versus $\left|Q_{j e t}\right|$, where the distributions have been fit to the function $D($ raw $)=\left|Q_{\text {jet }}\right| \cdot D_{\max }($ raw $)$.

Center left and right: the population of events in $\left|Q_{j e t}\right|$. The quoted efficiency is the total number of jet charge-tagged events divided by the total number of events in the sample.

Bottom left and right: $\epsilon D^{2}($ raw $)$ versus $\left|Q_{\text {jet }}\right|$.

The plots shown are taken from the Run 1B data samples. 

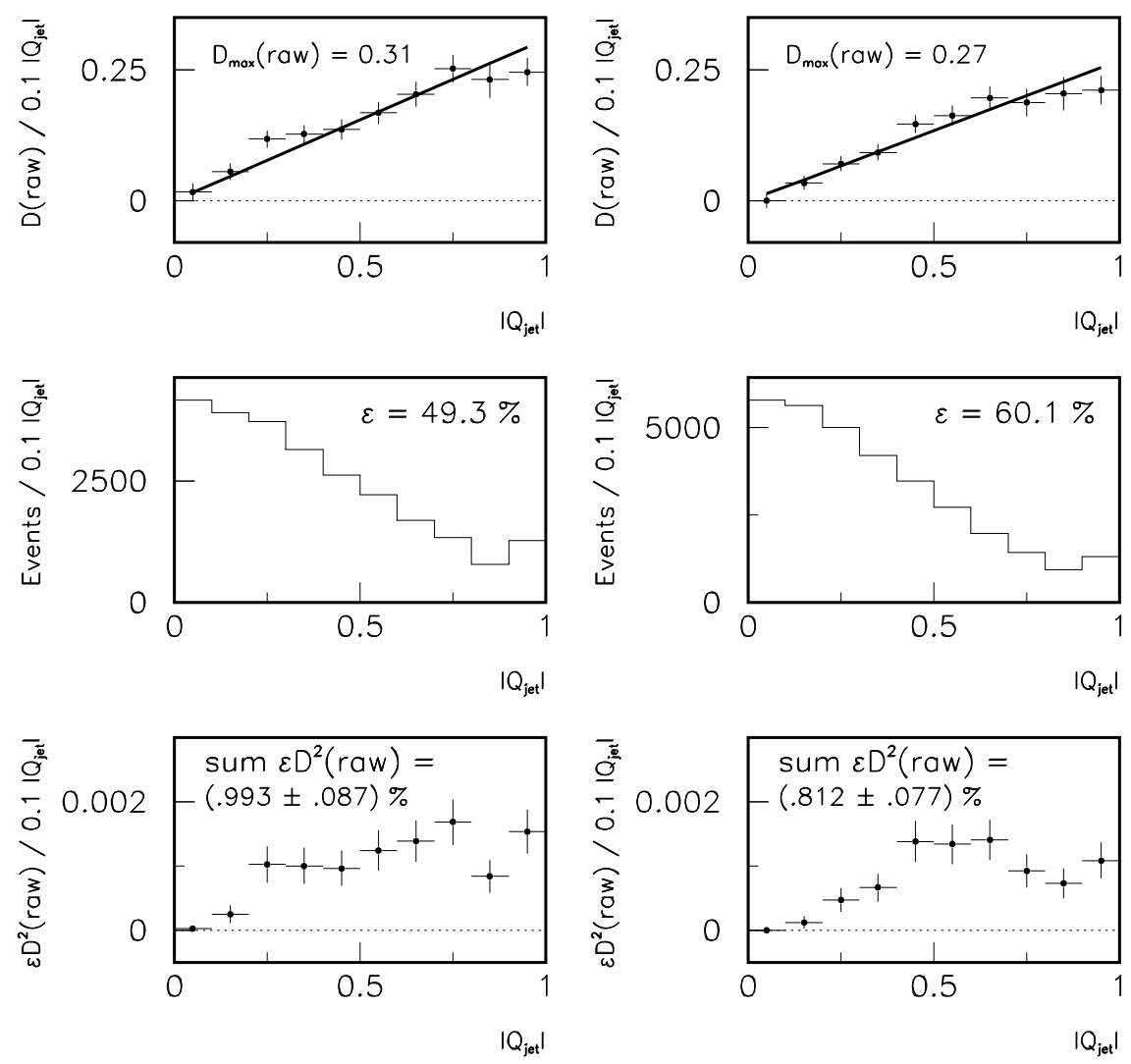

Figure 7.25: Jet charge flavor-tagging dilution, efficiency, and $\epsilon D^{2}$ in the $b \bar{b}$ Monte Carlo samples.

Top left and right: $D($ raw $)$ versus $\left|Q_{\text {jet }}\right|$, where the distributions have been fit to the function $D($ raw $)=\left|Q_{\text {jet }}\right| \cdot D_{\max }($ raw $)$.

Center left and right: the population of events in $\left|Q_{j e t}\right|$. The quoted efficiency is the total number of jet charge-tagged events divided by the total number of events in the sample.

Bottom left and right: $\epsilon D^{2}($ raw $)$ versus $\left|Q_{\text {jet }}\right|$.

The plots shown are taken from the Run 1B $b \bar{b}$ Monte Carlo samples. 
gives $A^{ \pm}=(0.88 \pm 0.14) \%$ for the combined trigger electron and muon data samples. Both the number of tracks and the charge asymmetry, as a function of $p_{\mathrm{t}}$, are shown in Figure 7.26. The Monte Carlo samples have a charge asymmetry consistent with zero.

The effect of the charge asymmetry can be investigated by artificially inducing an asymmetry into the Monte Carlo. The asymmetry in the data is parametrized as a function of $p_{\mathrm{t}}$ by an exponential, and used to calculate a negative track survival probability

$$
P^{-}\left(p_{\mathrm{t}}\right)=\frac{1-A^{ \pm}\left(p_{\mathrm{t}}\right)}{1+A^{ \pm}\left(p_{\mathrm{t}}\right)}
$$

The jet charge was recalculated in the Monte Carlo after comparing uniform random numbers to the survival probability to decide whether a negative track would be ignored. The loss of negative tracks biases the jet charge upward, as can be seen in Figure 7.27. The bias is quite small, but even so, the shift shown in Figure 7.27 is applied as a correction to the jet charge as calculated in the data samples. The raw jet charge dilutions supplied to the fit for $\Delta m_{d}$ incorporate this correction. 

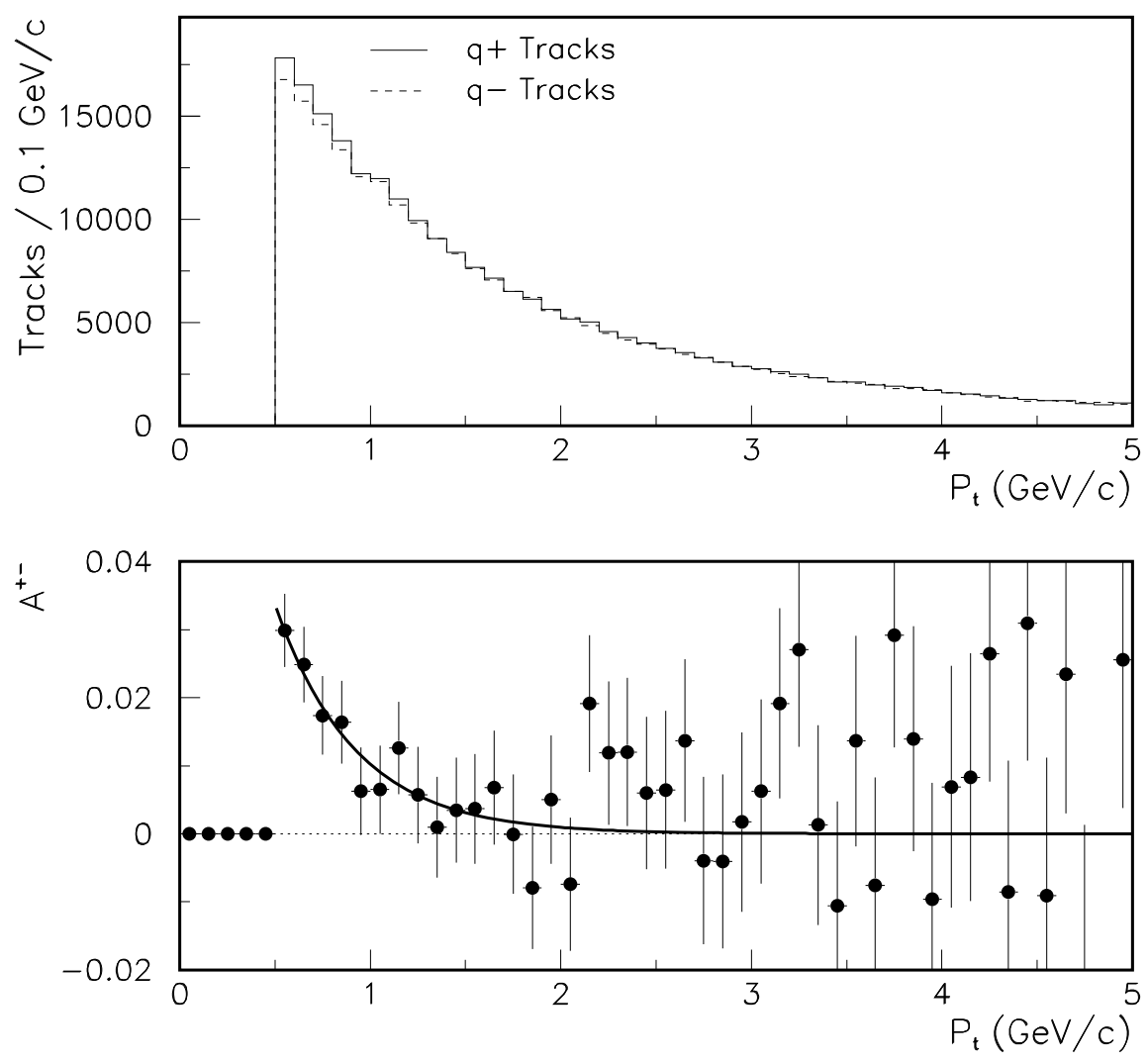

Figure 7.26: Top: the track $p_{\mathrm{t}}$ spectra for positively- and negatively-charged tracks. There are clearly more positive tracks than negative tracks at small $p_{\mathrm{t}}$.

Bottom: the asymmetry (defined in Equation 7.11) as a function of $p_{\mathrm{t}}$. The data is fit to an exponential. 


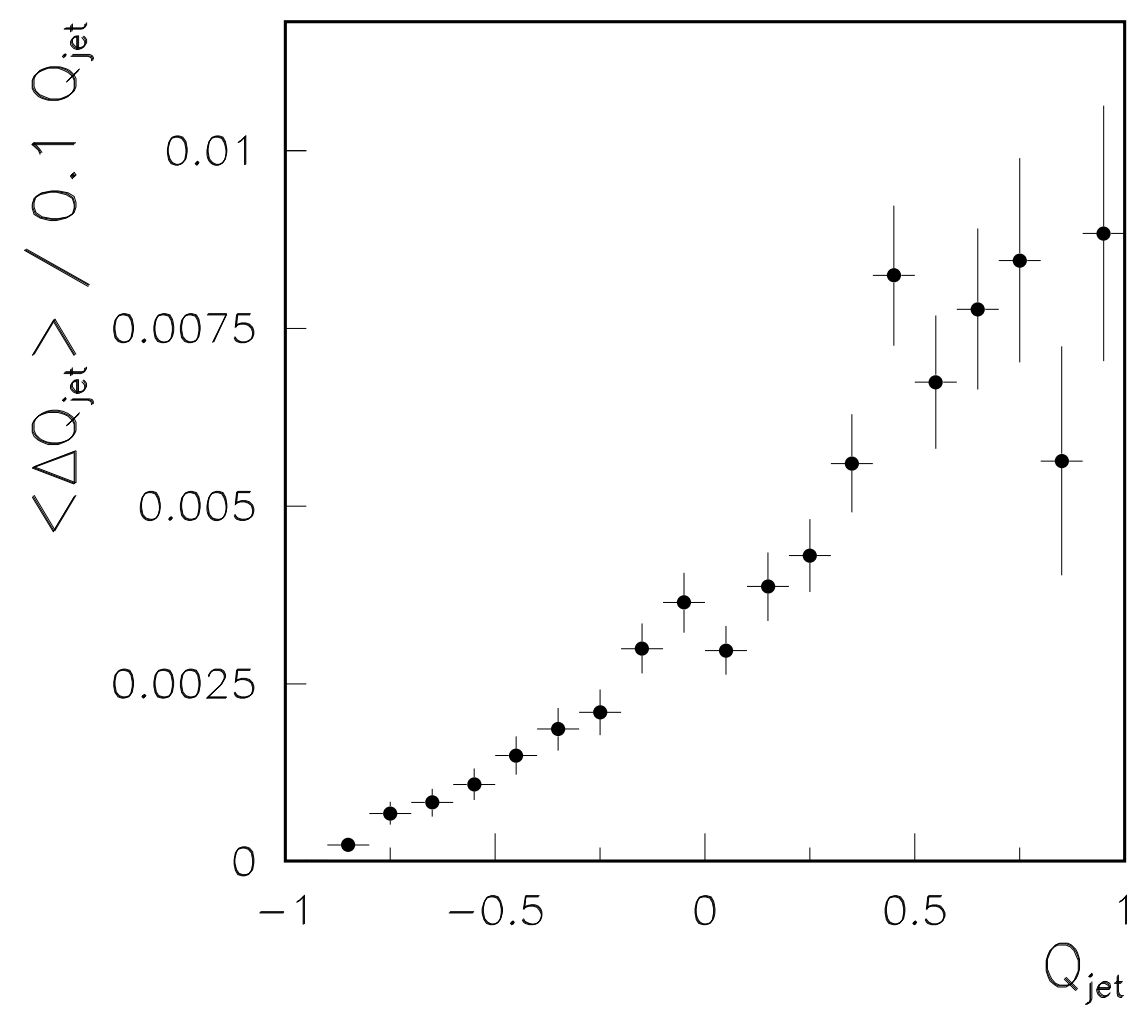

Figure 7.27: The average change in Monte Carlo $Q_{j e t}$ versus $Q_{j e t}$ after artificially introducing the charge asymmetry observed in the data. Though the change is never larger than $1 \%$, it is subtracted from the measured $Q_{j e t}$ in the data, to correct for the charge asymmetry. 


\subsection{Flavor-tagged Subsample Compositions}

It is possible that the presence of a flavor-tag could alter the sample composition. For example, the $p_{\mathrm{t}}$ spectrum of opposite side jets in $c \bar{c}$ events could be different enough from the $b \bar{b} p_{\mathrm{t}}$ spectrum that the $5 \mathrm{GeV} / \mathrm{c}$ jet $p_{\mathrm{t}}$ cut could noticeably reduce the charm fraction in the data samples. Therefore, the $p_{\mathrm{t}}^{\mathrm{rel}}$ and $M_{c l}$ fits were performed again on those subsamples of events having either a jet charge or an SLT flavor-tag.

The sample composition fit results for events with a soft lepton flavor-tag are shown numerically in Table 7.18, and and the $p_{\mathrm{t}}^{\text {rel }}$ fit results are shown graphically in Figure 7.28. The measured $b \bar{b}$ fractions are within the assigned systematic error of $3 \%$ of the fit to the entire sample. Since the $p_{\mathrm{t}}^{\mathrm{rel}}$ templates for $c \bar{c}$ and fakes are substantially similar, they tend to produce a more correlated fit result in the low statistics limit. The $p_{\mathrm{t}}^{\text {rel }}$ template fit is clearly unable to distinguish between the $c \bar{c}$ and fake lepton components, preferring to attribute low- $p_{\mathrm{t}}^{\text {rel }}$ events to fakes, rather than charm. The $M_{c l}$ fit distinguishes better between $c \bar{c}$ and fakes, giving good agreement with the fits to the entire samples (see Table 6.3). In this case, the difference between the $p_{\mathrm{t}}^{\mathrm{rel}}$ and $M_{c l}$ results will not be assigned as a systematic error to the sample compositions, but rather the $M_{c l}$ fit will be solely relied upon to assure that the composition is not substantially altered by the required presence of a soft lepton tag.

The composition of jet charge-tagged events is shown numerically in Table 7.19, and the $p_{\mathrm{t}}^{\text {rel }}$ fit results are shown graphically in Figure 7.29. As opposed to the soft lepton flavor-tagged subsample, both the $p_{\mathrm{t}}^{\mathrm{rel}}$ and $M_{c l}$ fits yield $f_{b \bar{b}}, f_{c \bar{c}}$, and $f_{\text {fake }}$ fraction which agree quite well with the previous results (Tables 6.1 and 6.3), within the assigned systematic error of $3 \%$. 


\begin{tabular}{|c||c|r|r|}
\hline & $f_{b \bar{b}}$ & \multicolumn{1}{c|}{$f_{c \bar{c}}$} & \multicolumn{1}{c|}{$f_{\text {fake }}$} \\
\hline \hline & \multicolumn{3}{|c|}{$p_{\mathrm{t}}^{\text {rel }}$ fit } \\
\hline$e:$ & $(83.6 \pm 3.3) \%$ & $(3.3 \pm 2.3) \%$ & $(13.1 \pm 4.0) \%$ \\
$\mu:$ & $(86.3 \pm 2.6) \%$ & $(3.8 \pm 1.8) \%$ & $(9.9 \pm 3.1) \%$ \\
\hline & \multicolumn{3}{|c}{$M_{c l}$ fit } \\
\hline$e:$ & $(88.8 \pm 2.4) \%$ & $(6.9 \pm 1.5) \%$ & $(4.3 \pm 3.0) \%$ \\
$\mu:$ & $(86.9 \pm 1.9) \%$ & $(12.1 \pm 1.3) \%$ & $(1.0 \pm 3.1) \%$ \\
\hline
\end{tabular}

Table 7.18: Results of the $p_{\mathrm{t}}^{\mathrm{rel}}$ and $M_{c l}$ fits to the subsamples of events with a soft lepton flavor-tag.
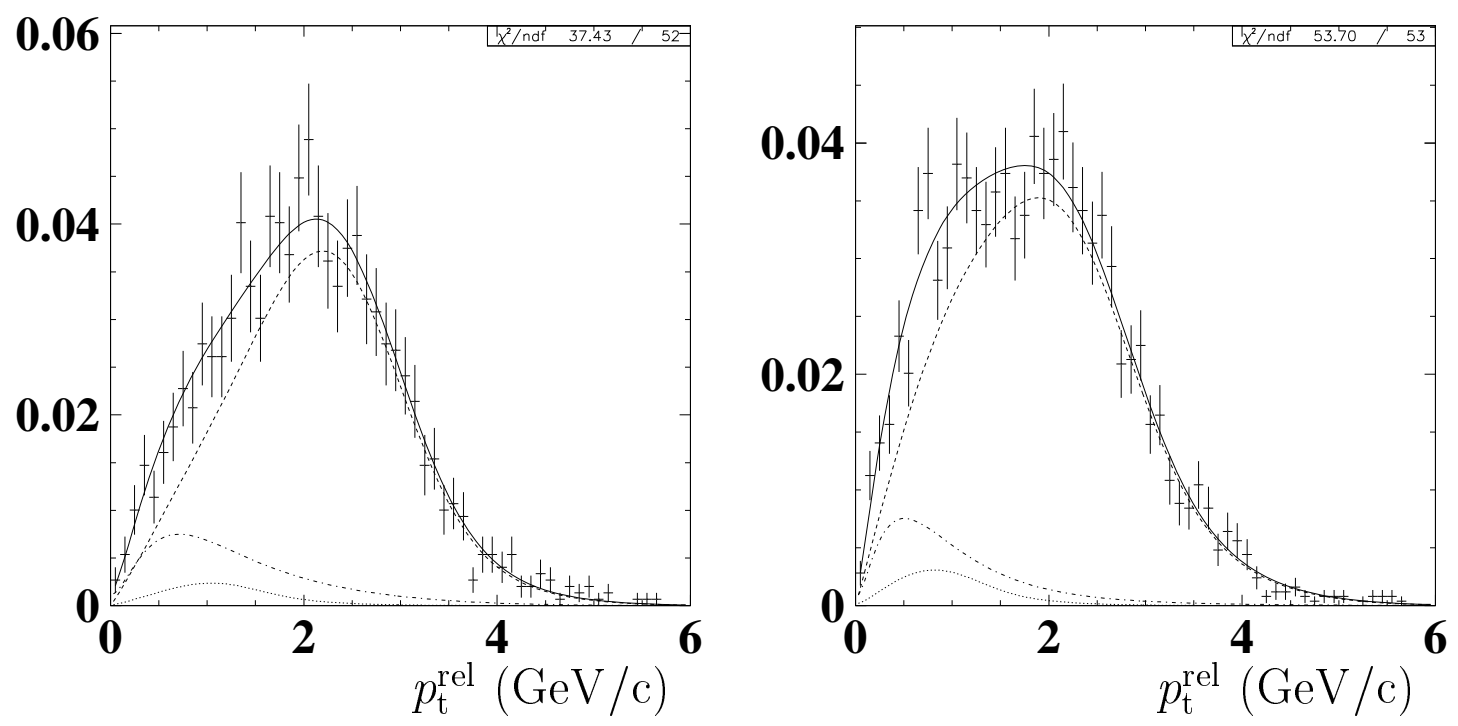

Figure 7.28: Results of the $p_{\mathrm{t}}^{\text {rel }}$ fits to events with a soft lepton tag for the trigger electron (left) and trigger muon (right) samples. The contributions from $b \bar{b}$ (dashed), $c \bar{c}$ (dotted), and fake leptons (dashed-dotted) are also shown. 


\begin{tabular}{|c||c|r|c|}
\hline & $f_{b \bar{b}}$ & $f_{c \bar{c}}$ & $f_{\text {fake }}$ \\
\hline \hline & \multicolumn{3}{|c|}{$p_{\mathrm{t}}^{\text {rel }}$ fit } \\
\hline$e:$ & $(85.1 \pm 1.0) \%$ & $(8.1 \pm 1.9) \%$ & $(6.5 \pm 2.0) \%$ \\
$\mu:$ & $(83.1 \pm 0.8) \%$ & $(13.8 \pm 1.5) \%$ & $(3.1 \pm 1.4) \%$ \\
\hline & \multicolumn{3}{|c}{$M_{c l}$ fit } \\
\hline$e:$ & $(88.6 \pm 0.9) \%$ & $(8.2 \pm 0.6) \%$ & $(3.0 \pm 1.0) \%$ \\
$\mu:$ & $(80.5 \pm 1.5) \%$ & $(15.2 \pm 0.8) \%$ & $(3.9 \pm 2.0) \%$ \\
\hline
\end{tabular}

Table 7.19: Results of the $p_{\mathrm{t}}^{\mathrm{rel}}$ and $M_{c l}$ fits to the subsamples of events with a jet charge flavor-tag.
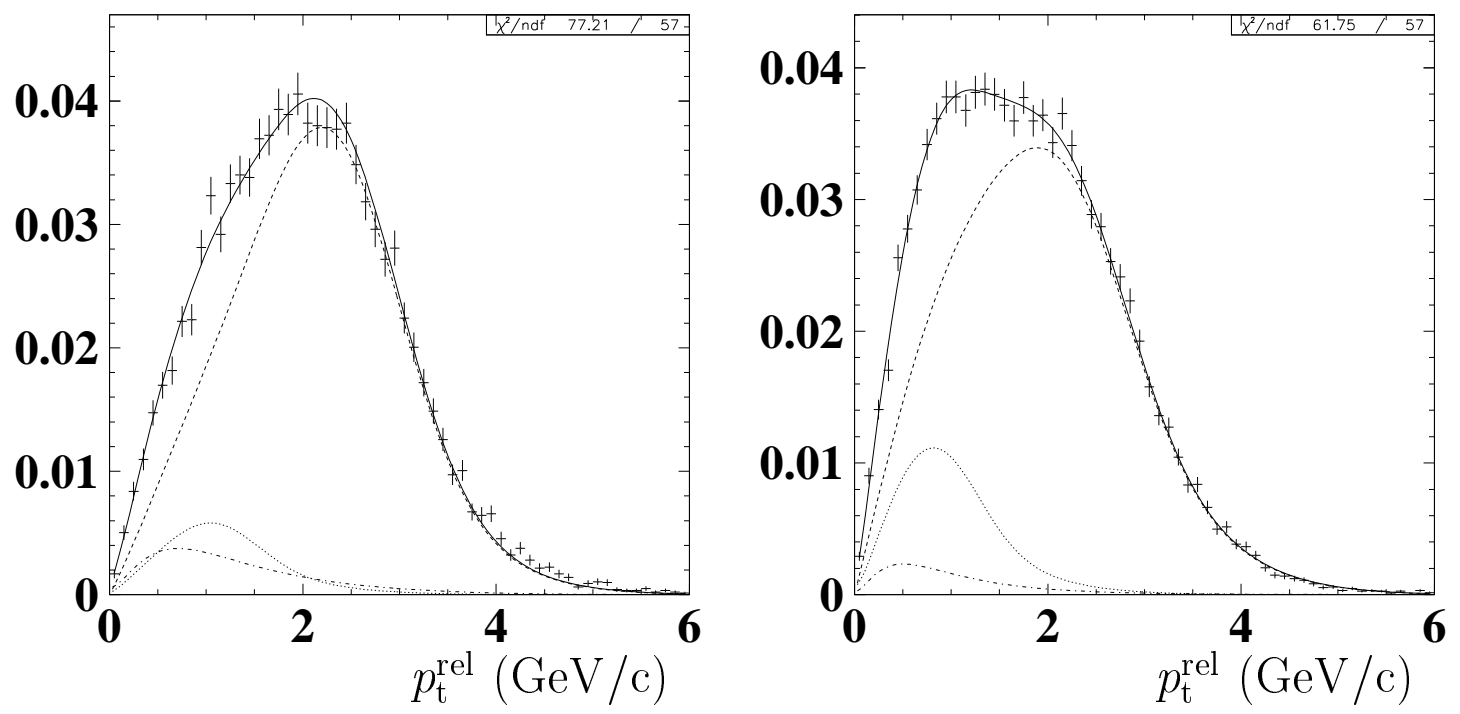

Figure 7.29: Results of the $p_{\mathrm{t}}^{\text {rel }}$ fits to events with a jet charge tag for the trigger electron (left) and trigger muon (right) samples. The contributions from $b \bar{b}$ (dashed), $c \bar{c}$ (dotted), and fake leptons (dashed-dotted) are also shown. 


\subsection{Dilution in $c \bar{c}$ and Fake Events}

The interpretation of the raw dilutions that have been presented is not simple. The raw dilutions are defined as the asymmetry between opposite- and same-sign trigger-tag combinations; for $b \bar{b}$ events, this definition therefore contains a contribution from the occasions when the trigger lepton comes from a mixed neutral $B$, or otherwise does not correctly identify the $b$ flavor. Mixing on the trigger side is not a genuine concern, as this is precisely what this analysis aims to measure, but fakes and sequentials are legitimate problems. In the case of $c \bar{c}$ events, as there is no contribution from mixing or sequential decays, all events should be found to be opposite-sign, in the limit that the flavor-taggers work perfectly. Finally, the correlation of fake leptons (which are primarily from $b$ hadron decay products) to their parent $b$ flavor must be understood. The raw SLT and jet charge dilutions from the data given previously represent an integration over all of these effects, given that the sample composition studies have measured nonzero $c \bar{c}$ and fake events in the data.

\subsection{1 $c \bar{c}$ Dilution}

With the current $c \bar{c}$ Monte Carlo samples, no reliable study of the SLT dilution in $c \bar{c}$ events has been done. As there are no sequential decays, the largest source of improperly-correlated soft leptons in charm events is thought to be fake leptons. The ability of the Monte Carlo to accurately simulate the lepton fake rate is dubious at best. Even if it were trusted, the requirement that all ground-state charmed hadrons decay semileptonically in the $c \bar{c}$ generation guarantees that there is a real lepton from the second $c$ quark in every event and has the additional effects of altering the $\pi: K$ ratio and reducing the hadron multiplicity, obviating any chance of 
an effective fake rate simulation. Therefore, the assumption is made that the SLT dilution in charm events is 0.5 , and will be varied from 0 to 1 in the calculation of the systematic uncertainty, representing the lack of any knowledge of the real charm dilution. This (perhaps overly-) conservative position will be the largest contribution to the systematic uncertainty on $\Delta m_{d}$. This is considered acceptable, as the statistical error still dominates the determination of $\Delta m_{d}$ (as shown in Section 8.2).

In the case of jet charge flavor-tagging, the forced decays in the charm sample do not immediately preclude further study, but there is another immediate concern: the measured dilution is clearly higher in the $b \bar{b}$ Monte Carlo than the data (see Figures 7.24 and 7.25). It is possible that this is due to the differences in the $N_{\text {trk }}^{\text {jet }}$ track multiplicity between data and Monte Carlo shown in Section 6.2.1; it has been determined that it is not due to the low- $p_{\mathrm{t}}$ track charge asymmetry just discussed. Since the dilution normalization in the Monte Carlo cannot be completely trusted, the $c \bar{c}$ dilution will be determined relative to the $b \bar{b}$ dilution. While the $b \bar{b}$ raw dilution includes contributions from trigger $b$ mixing and sequential decays, the $c \bar{c}$ raw dilution does not. Consequently, the proper comparison to be made is not between the raw $b \bar{b}$ and $c \bar{c}$ dilutions, but rather between the true $b \bar{b}$ dilution and the raw $c \bar{c}$ dilution. The true $b \bar{b}$ dilution is calculated from the correlation of the jet charge flavor-tag with the production flavor of the trigger $b$ (taken from the event generation). It therefore avoids contributions from the imperfect correlation between the charge of the trigger lepton and the $b$ production flavor.

Figure 7.30 shows these distributions for the electron and muon Monte Carlo samples, along with the linear parametrization of the dilution. The ratio $D_{c \bar{c}} / D_{b \bar{b}}$ is $0.15 \pm 0.04$ and $0.26 \pm 0.04$ for the electron and muon samples, respectively. The "spikes" in the $c \bar{c}$ raw dilution at $\left|Q_{\text {jet }}\right|=1$ are due to the forcing of charmed hadrons to decay semileptonically in the event generation. Accordingly, while these 
ratios will be taken for the central values for the $\Delta m_{d}$ fit, they will be varied from 0 to 1 in the calculation of the systematic errors, which should amply cover any bias from the event generation.
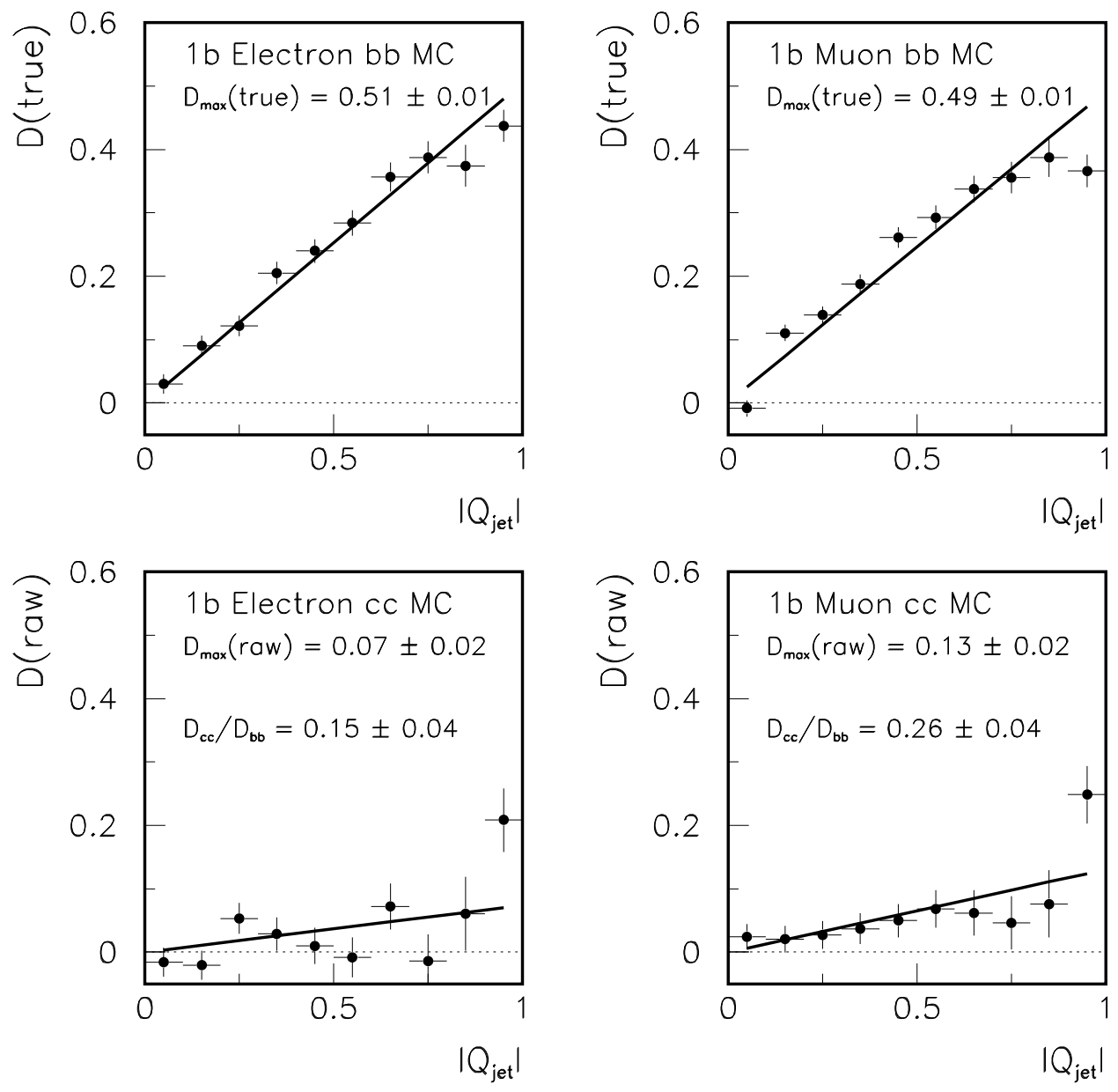

Figure 7.30: Top left and right: The true dilution versus $\left|Q_{\text {jet }}\right|$ for $b \bar{b}$ Monte Carlo, for the electron and muon samples. Bottom left and right: The raw dilution versus $\left|Q_{j e t}\right|$ for $c \bar{c}$ Monte Carlo, for the electron and muon samples. The relative dilution $D_{c \bar{c}} / D_{b \bar{b}}$ is taken to be the ratio of the $D_{\max }$ fit parameters. The true dilution for the $b \bar{b}$ Monte Carlo was obtained by comparing the flavor-tag with the generated flavor of the trigger $b$ hadron. 


\subsubsection{Fake Dilution}

As described in Section 6.1, fake leptons are assumed to be $b$ hadron decay products. Consequently, one would expect the flavor tags to work as well in the opposite $b$ jet in fake lepton events as it does in the real lepton events of the data samples. Any difference in raw dilution between the two can be attributed to the lesser flavorcharge correlation between the fake lepton and its parent $b$ hadron compared to that of real leptons and their parents.

Thus the raw dilution in the fake lepton samples can be parametrized as was done for the data samples. Figure 7.31 compares the raw jet charge dilutions plotted versus $\left|Q_{\text {jet }}\right|$ for the electron and muon samples. The raw dilution in the fake events is not much smaller than the the raw dilution in the data. This is not as surprising as one might think. Intuition suggests that the leading kaon from the $b \rightarrow c \rightarrow s$ decay chain may be the most likely $b$ decay product to become a fake lepton ${ }^{4}$. If this is true, then the size of the raw dilution in fake events is to be expected, since the charge of the leading kaon is correlated with the flavor of its $b$ grandparent (see Section 7.3). It should be noted, however, that the parametrization of the fake dilution does not depend on whether this intuition is correct.

While this parametrization could be used directly in the fit for $\Delta m_{d}$, the low statistics of the SECVTX-tagged fake samples give one pause. Instead, the ratio of the fake to the data dilution will be used, as it is in the case of the jet charge dilution in charm events. The ratio is called $D_{\text {fake }} / D_{b \bar{b}}$, although it should be stressed that the ratio is derived from the two data samples as described, and does not involve any Monte Carlo simulation despite the use of the subscript " $b \bar{b}$ ". The ratio is $0.92 \pm 0.26$ and $0.71 \pm 0.17$ for the electron and muon samples, respectively. As in the case of the charm dilution, the ratio is varied from 0 to 1 in the calculation of

\footnotetext{
${ }^{4}$ See Section 7.2.5 for a discussion of how kaons may fake lepton signatures.
} 
the systematic uncertainties. Though the uncertainty on the electron ratio would indicate the potential for a ratio larger than one, it is believed that the fake lepton dilution cannot be larger than the real lepton dilution.

The $D_{f a k e} / D_{b \bar{b}}$ ratio for soft lepton flavor-tagging is difficult to determine. The number of SLT-tagged fake events is too small to give a statistically significant measurement of the ratio. Accordingly, the same assumption made for the SLT $D_{c \bar{c}} / D_{b \bar{b}}$ is made for $D_{f a k e} / D_{b \bar{b}}$. That is, $D_{f a k e} / D_{b \bar{b}}=0.5$ and varied from 0 to 1 in the calculation of the systematic uncertainty. Unlike the case of the charm SLT dilution, however, this variation has a negligible contribution to the total error. 

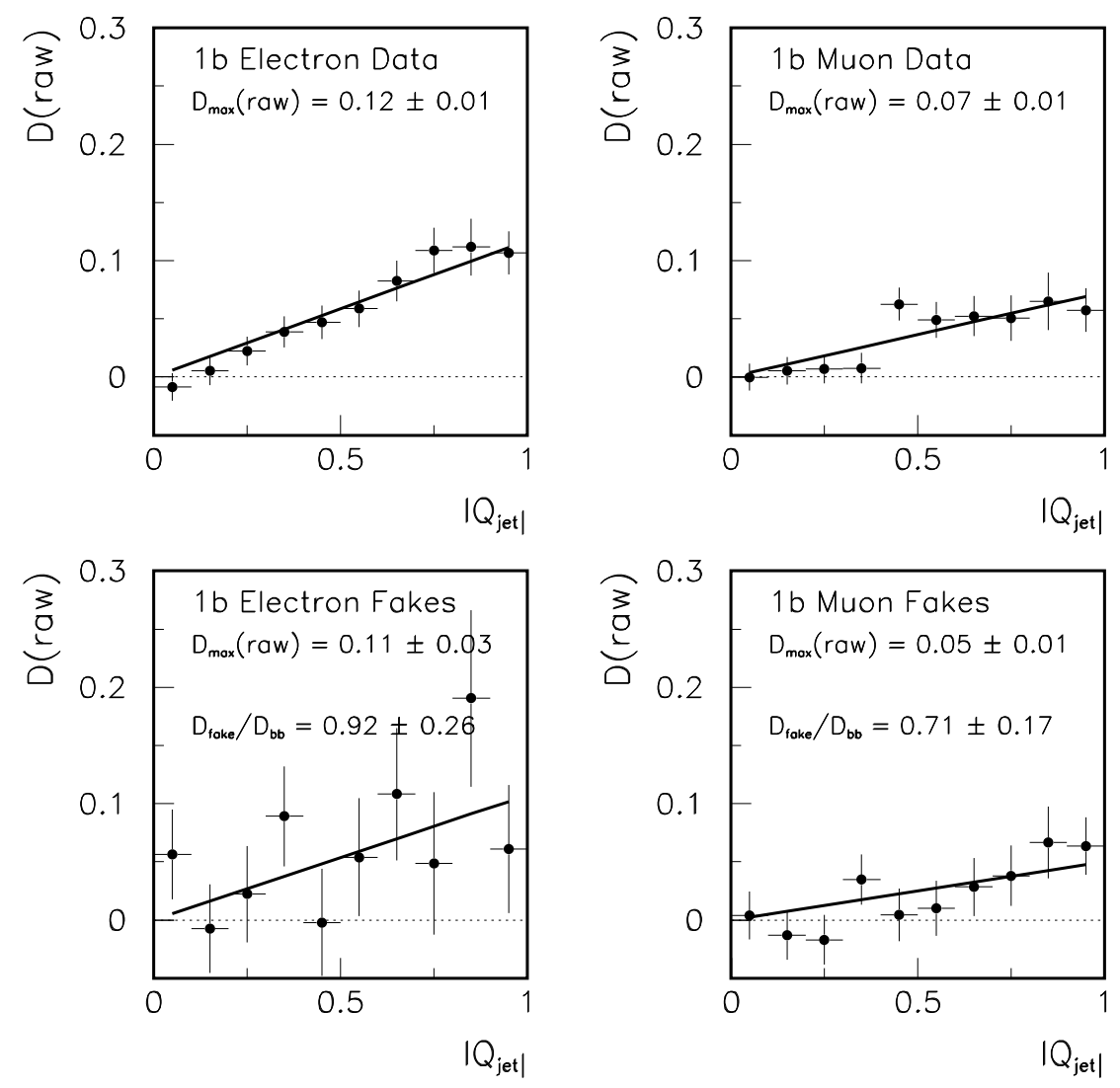

Figure 7.31: Top left and right: The $D($ raw $)$ versus $\left|Q_{\text {jet }}\right|$ for the data samples. Bottom left and right: The $D($ raw $)$ versus $\left|Q_{\text {jet }}\right|$ for the fake lepton samples.

The relative dilution $D_{f a k e} / D_{b \bar{b}}$ is defined as the ratio of the fit $D_{\max }(\mathrm{raw})$ parameters. 


\section{Chapter 8}

\section{Measuring $\Delta m_{d}$}

The necessary elements for a measurement of $\Delta m_{d}$ have been collected: the SECVTX $L_{x y}, p_{\mathrm{t}}^{c l}$, and $M_{c l}$ give the proper time of the trigger $b$ hadron decay, and the correlation of the trigger lepton charge with the SLT or jet charge flavortag gives the flavor of the trigger $b$ hadron at its production and decay. The Monte Carlo and fake samples give the fraction of events in the data samples from $b \bar{b}, \mathrm{ccb}$, and fake leptons, and provide additional information on contributions to the raw dilution, whether on the trigger side, through sequentials and fakes, or on the tag side, with an estimate of the $c \bar{c}$ jet charge dilution. The time-dependence of $B_{d}^{0}$ mixing can then be directly measured with an unbinned maximum-likelihood fit, along with the oscillation amplitude. The fit procedure is tested with a fast Monte Carlo (FMC), based upon data distributions, to check both the fit systematics and the scale of the errors and total likelihood returned by the fit.

\subsection{The Maximum-Likelihood Fit}

The maximum-likelihood method is a powerful technique for estimating the parameters $(\theta)$ of the probability density function $f\left(x_{i} \mid \theta\right)$ from which a set of independent 
observations $\left(x_{i}\right)$ were made. The joint probability density, called the likelihood, for all the data is

$$
\mathcal{L}(\theta) \equiv \prod_{i=1}^{n} f\left(x_{i} \mid \theta\right)
$$

The principle of the method is that the most probable values of $\theta$ are those which maximize the likelihood. The negative $\log$ likelihood $(-\ln \mathcal{L})$ is typically evaluated instead of $\mathcal{L}$. Since $\mathcal{L}$ and $\ln \mathcal{L}$ are both maximized for the same $\theta$, the logarithm is used because a sum is computationally simpler than a product. The negative of the $\log$ likelihood is used for convenience, since most popular fitting software packages are minimizers rather than maximizers.

The probability density has two free parameters and many fixed parameters. The first free parameter is $\Delta m_{d}$. The second is the raw dilution normalization $N_{D}$. The argument for including this second free parameter is as follows:

The true flavor-tagging dilution is the measure of the probability of correctly identifying the production flavor of the trigger $b$ quark. The raw dilution provided by the jet charge and SLT flavor-taggers is based on the correlation of the flavortag with the charge of the trigger lepton, as discussed in Section 7.5. The two are related by the normalization factor $N_{D}$ :

$$
D=N_{D} \cdot D(r a w)
$$

$N_{D}$ corrects for the occasions when the second $b$ hadron is a neutral $B$ meson which has mixed as well as for the occasions when the trigger lepton does not have the correct charge correlation with its parent quark. The former depends on $\Delta m_{d}$; the latter can be determined from the sample composition. It is therefore possible to construct a probability density function which depends on $\Delta m_{d}$ alone, but there is a good reason for not doing so. 
A measurement of $B_{d}^{0}$ mixing should be sensitive to both the frequency and the amplitude of the mixing oscillation. The frequency depends only on $\Delta m_{d}$; the amplitude depends on a combination of $\Delta m_{d}$, the raw dilution, and the sample composition. By making $N_{D}$ a free parameter, the fit for $\Delta m_{d}$ is sensitive only to the frequency. It will be shown in Section 8.2 that this decouples the systematic uncertainties on the sample composition from the measurement of $\Delta m_{d}$, resulting in a more precise measurement.

The fixed parameters of the probability density are the following: the fractions of $b \bar{b}, c \bar{c}$, and fake events, the $b$ hadron lifetimes, the $b$ hadron species fractions and the wrong-sign sequential fractions for the $b \bar{b}$ events, the flavor-tagging dilutions, the SECVTX efficiency versus $c \tau$, the value of $\Delta m_{s}$, and the $\mathrm{c} \tau$ correction and resolution. Each of these parameters will be varied within their uncertainties to determine their contribution to the systematic uncertainty on $\Delta m_{d}$ and $N_{D}$.

The probability density must be a function of only observable quantities, except for the two free parameters. It is first derived from Standard Model predictions as a function of the true proper time. Accordingly, the probability density must be convoluted with the experimental $\mathrm{c} \tau$ resolution to tranform it into a function of the reconstructed proper time.

\subsubsection{The Probability Density}

An event is classified as same-sign or opposite-sign by comparing the sign of the flavor-tag with the sign of the trigger lepton charge. Two very similar probability density functions must be developed: one for the set of all same-sign events and one for the set of all opposite-sign events.

Consider the simple case of a pure $B_{d}^{0}$ sample that is free of sequential decays. A $B_{d}^{0}$ meson that has not yet decayed has a time-dependent mixing probability given 
by

$$
P_{\text {mixed }}^{B_{d}^{0}}\left(\mathrm{c} \tau_{0}\right)=\frac{1}{2}\left(1-\cos \left(\Delta m_{d} \mathrm{c} \tau_{0}\right)\right)
$$

The probability that it has not mixed is

$$
P_{\text {unmixed }}^{B_{d}^{0}}\left(\mathrm{c} \tau_{0}\right)=\frac{1}{2}\left(1+\cos \left(\Delta m_{d} \mathrm{c} \tau_{0}\right)\right)
$$

$\mathrm{c} \tau_{0}$ is the proper time and $\Delta m_{d}$ the mixing parameter. A flavor-tagger must be used to determine whether or not the $B_{d}^{0}$ has mixed before its decay. Given a flavortagging dilution $D$, the probability that the mixed or unmixed state of an event was tagged correctly is

$$
P_{t a g}(D)=\frac{1}{2}(1+D)
$$

and the probability that the state was tagged incorrectly is

$$
P_{\text {mistag }}(D)=\frac{1}{2}(1-D)
$$

The dilution is equal to the raw dilution times the dilution normalization factor $N_{D}$, according to Equation 8.2.

The probability densities for same- and opposite-sign events are constructed from the combination of the $B_{d}^{0}$ lifetime and the mixing and flavor-tagging probabilities. They are given by

$$
\begin{aligned}
P_{S S}\left(\mathrm{c} \tau_{0}, D: B_{d}^{0}\right)= & \frac{1}{\mathrm{c} \tau_{B_{d}^{0}}} \cdot e^{-\frac{\mathrm{c} \tau_{0}}{\mathrm{c} \tau_{d}^{0}}} \\
& \times\left[P_{\text {tag }}(D) \cdot P_{\text {mixed }}^{B_{d}^{0}}\left(\mathrm{c} \tau_{0}\right)+P_{\text {mistag }}(D) \cdot P_{\text {unmixed }}^{B_{d}^{0}}\left(\mathrm{c} \tau_{0}\right)\right] \\
P_{O S}\left(\mathrm{c} \tau_{0}, D: B_{d}^{0}\right)= & \frac{1}{\mathrm{c} \tau_{B_{d}^{0}}} \cdot e^{-\frac{\mathrm{c} \tau_{0}}{\mathrm{c}{ }_{B_{d}^{0}}^{0}}} \\
& \times\left[P_{\text {tag }}(D) \cdot P_{\text {unmixed }}^{B_{d}^{0}}\left(\mathrm{c} \tau_{0}\right)+P_{\text {mistag }}(D) \cdot P_{\text {mixed }}^{B_{d}^{0}}\left(\mathrm{c} \tau_{0}\right)\right]
\end{aligned}
$$


The probability densities are correctly normalized:

$$
P_{S S}\left(\mathrm{c} \tau_{0}, D: B_{d}^{0}\right)+P_{O S}\left(\mathrm{c} \tau_{0}, D: B_{d}^{0}\right)=\frac{1}{\mathrm{c} \tau_{B_{d}^{0}}} \cdot e^{-\frac{\mathrm{c} \tau_{0}}{\mathrm{c} \tau_{d}^{0}}}
$$

and

$$
\int_{0}^{\infty}\left[P_{S S}\left(\mathrm{c} \tau_{0}, D: B_{d}^{0}\right)+P_{O S}\left(\mathrm{c} \tau_{0}, D: B_{d}^{0}\right)\right] d \mathrm{c} \tau_{0}=1
$$

The probability density for $B_{s}^{0}$ mesons in the absence of sequentials has the same form, with its own lifetime and mixing parameter $\left(\Delta m_{s}\right)$ replacing those of the $B_{d}^{0}$. $\Delta m_{s}$ is fixed in the fit, since the the SECVTX $\mathrm{c} \tau$ efficiency turn-on leaves the sample fairly insensitive to $B_{s}^{0}$ mixing. The densities for the $b$ baryons and charged $B$ mesons are simpler since they cannot mix:

$$
\begin{aligned}
P_{S S}\left(\mathrm{c} \tau_{0}, D: B_{u}\right) & =\frac{1}{\mathrm{c} \tau_{B_{u}}} \cdot e^{-\frac{\mathrm{c} \tau_{0}}{c \tau_{u}}}\left[P_{\text {mistag }}(D)\right] \\
P_{O S}\left(\mathrm{c} \tau_{0}, D: B_{u}\right) & =\frac{1}{\mathrm{c} \tau_{B_{u}}} \cdot e^{-\frac{\mathrm{c} \tau_{0}}{c \tau_{B}}}\left[P_{t a g}(D)\right] \\
P_{S S}\left(\mathrm{c} \tau_{0}, D: \Lambda_{b}\right) & =\frac{1}{\mathrm{c} \tau_{\Lambda_{b}}} \cdot e^{-\frac{\mathrm{c} \tau_{0}}{c \tau_{b}}}\left[P_{\text {mistag }}(D)\right] \\
P_{O S}\left(\mathrm{c} \tau_{0}, D: \Lambda_{b}\right) & =\frac{1}{\mathrm{c} \tau_{\Lambda_{b}}} \cdot e^{-\frac{c \tau_{0}}{c \tau_{b}}}\left[P_{t a g}(D)\right]
\end{aligned}
$$

The total $b \bar{b}$ probability density in the absence of sequentials is constructed from the individual $b$ hadron densities, accounting for the fractions of each species present (to be discussed in Section 8.1.4):

$$
\begin{aligned}
P_{S S}^{*}\left(\mathrm{c} \tau_{0}, D: b \bar{b}\right)= & F_{B_{d}^{0}}^{\prime} \cdot P_{S S}\left(\mathrm{c} \tau_{0}, D: B_{d}^{0}\right)+F_{B_{s}^{0}}^{\prime} \cdot P_{S S}\left(\mathrm{c} \tau_{0}, D: B_{s}^{0}\right)+ \\
& F_{B_{u}}^{\prime} \cdot P_{S S}\left(\mathrm{c} \tau_{0}, D: B_{u}\right)+F_{\Lambda_{b}}^{\prime} \cdot P_{S S}\left(\mathrm{c} \tau_{0}, D: \Lambda_{b}\right) \\
P_{O S}^{*}\left(\mathrm{c} \tau_{0}, D: b \bar{b}\right)= & F_{B_{d}^{0}}^{\prime} \cdot P_{O S}\left(\mathrm{c} \tau_{0}, D: B_{d}^{0}\right)+F_{B_{s}^{0}}^{\prime} \cdot P_{O S}\left(\mathrm{c} \tau_{0}, D: B_{s}^{0}\right)+ \\
& F_{B_{u}}^{\prime} \cdot P_{O S}\left(\mathrm{c} \tau_{0}, D: B_{u}\right)+F_{\Lambda_{b}}^{\prime} \cdot P_{O S}\left(\mathrm{c} \tau_{0}, D: \Lambda_{b}\right)
\end{aligned}
$$




\section{Including Sequentials}

The wrong-sign sequential fraction as a function of true $\mathrm{c} \tau$ has been measured in the Monte Carlo (see Figure 5.6). Nevertheless, including it directly in the likelihood, the correct thing to do, will render the $\mathrm{c} \tau$-resolution convolution difficult to perform. As $P_{w s}^{s e q}$ varies slowly on the scale of the uncertainty on $\mathrm{c} \tau$, the fraction can be taken outside the integral and included in the probability density as a function of the reconstructed $\mathrm{c} \tau$ (also shown in Figure 5.6).

Wrong-sign sequentials mis-identify the flavor of $b$ hadrons at their decay. Thus they "confuse" the same- and opposite-sign densities which have been constructed so far:

$$
\begin{aligned}
P_{S S}\left(\mathrm{c} \tau_{0}, \mathrm{c} \tau_{r}, D: b \bar{b}\right)= & {\left[1-P_{w s}^{s e q}\left(\mathrm{c} \tau_{r}\right)\right] \cdot P_{S S}^{*}\left(\mathrm{c} \tau_{0}, D: b \bar{b}\right)+} \\
& P_{w s}^{s e q}\left(\mathrm{c} \tau_{r}\right) \cdot P_{O S}^{*}\left(\mathrm{c} \tau_{0}, D: b \bar{b}\right) \\
P_{O S}\left(\mathrm{c} \tau_{0}, \mathrm{c} \tau_{r}, D: b \bar{b}\right)= & {\left[1-P_{w s}^{s e q}\left(\mathrm{c} \tau_{r}\right)\right] \cdot P_{O S}^{*}\left(\mathrm{c} \tau_{0}, D: b \bar{b}\right)+} \\
& P_{w s}^{s e q}\left(\mathrm{c} \tau_{r}\right) \cdot P_{S S}^{*}\left(\mathrm{c} \tau_{0}, D: b \bar{b}\right)
\end{aligned}
$$

\section{Including Charm and Fake Leptons}

The $c \bar{c}$ component has the same form as the non-mixing $b$ hadron terms, albeit with no sequentials:

$$
\begin{aligned}
& P_{S S}\left(\mathrm{c} \tau_{0}, D, D_{c \bar{c}} / D_{b \bar{b}}: c \bar{c}\right)=\frac{1}{\mathrm{c} \tau_{c \bar{c}}} \cdot e^{-\frac{c \tau_{0}}{c \tau_{c \bar{c}}}} \cdot\left[P_{m i s t a g}\left(D \cdot D_{c \bar{c}} / D_{b \bar{b}}\right)\right] \\
& P_{O S}\left(\mathrm{c} \tau_{0}, D, D_{c \bar{c}} / D_{b \bar{b}}: c \bar{c}\right)=\frac{1}{\mathrm{c} \tau_{c \bar{c}}} \cdot e^{-\frac{c \tau_{0}}{c \tau_{c \bar{c}}}} \cdot\left[P_{t a g}\left(D \cdot D_{c \bar{c}} / D_{b \bar{b}}\right)\right]
\end{aligned}
$$

The charm dilution must be handled separately for the jet charge- and SLT-tagged events, as discussed in Section 7.5.1. The ratio $D_{c \bar{c}} / D_{b \bar{b}}$ is applied to $D$ only for jet charge-tagged events. The product $D \cdot D_{c \bar{c}} / D_{b \bar{b}}$ is taken to be 0.5 for SLT-tagged 
events. In neither case will the normalization factor $N_{D}$ be included in $D$.

Section 6.1 described how the fake lepton $\mathrm{c} \tau$ distribution led to the assumption that all fake leptons are $b$ decay products, and Section 7.5.2 showed the dilution in the fake samples is found to be lower by some factor relative to the data samples. Accordingly, the fake lepton probability densities are the same as those for the $b$ densities, with a scaled dilution:

$$
\begin{aligned}
& P_{S S}\left(\mathrm{c} \tau_{0}, \mathrm{c} \tau_{r}, D, D_{\text {fake }} / D_{b \bar{b}}: \text { fake }\right)=P_{S S}\left(\mathrm{c} \tau_{0}, \mathrm{c} \tau_{r}, D \cdot D_{\text {fake }} / D_{b \bar{b}}: b \bar{b}\right) \\
& P_{O S}\left(\mathrm{c} \tau_{0}, \mathrm{c} \tau_{r}, D, D_{\text {fake }} / D_{b \bar{b}}: \text { fake }\right)=P_{O S}\left(\mathrm{c} \tau_{0}, \mathrm{c} \tau_{r}, D \cdot D_{\text {fake }} / D_{b \bar{b}}: b \bar{b}\right)
\end{aligned}
$$

As these are $b \bar{b}$ events, the normalization factor $N_{D}$ is included in $D$.

\section{Total Probability Density}

The total same- and opposite-sign probability densities can be constructed from the previous components and the sample composition fractions, accordingly:

$$
\begin{gathered}
P_{S S}\left(\mathrm{c} \tau_{0}, \mathrm{c} \tau_{r}, D, D_{c \bar{c}} / D_{b \bar{b}}, D_{\text {fake }} / D_{b \bar{b}}\right)= \\
f_{b \bar{b}} \cdot P_{S S}\left(\mathrm{c} \tau_{0}, \mathrm{c} \tau_{r}, D: b \bar{b}\right)+ \\
f_{c \bar{c}} \cdot P_{S S}\left(\mathrm{c} \tau_{0}, D, D_{c \bar{c}} / D_{b \bar{b}}: c \bar{c}\right)+ \\
f_{f a k e} \cdot P_{S S}\left(\mathrm{c} \tau_{0}, \mathrm{c} \tau_{r}, D, D_{f a k e} / D_{b \bar{b}}: \text { fake }\right) \\
P_{O S}\left(\mathrm{c} \tau_{0}, \mathrm{c} \tau_{r}, D, D_{c \bar{c}} / D_{b \bar{b}}, D_{f a k e} / D_{b \bar{b}}\right)= \\
f_{b \bar{b}} \cdot P_{O S}\left(\mathrm{c} \tau_{0}, \mathrm{c} \tau_{r}, D: b \bar{b}\right)+ \\
f_{c \bar{c}} \cdot P_{O S}\left(\mathrm{c} \tau_{0}, D, D_{c \bar{c}} / D_{b \bar{b}}: c \bar{c}\right)+ \\
f_{f a k e} \cdot P_{O S}\left(\mathrm{c} \tau_{0}, \mathrm{c} \tau_{r}, D, D_{f a k e} / D_{b \bar{b}}: \text { fake }\right)
\end{gathered}
$$




\subsubsection{Convolution with the $\mathrm{c} \tau$ Resolution}

To be properly evaluated for the data, the probability density must be made a function of only observable variables. Thus the true $\mathrm{c} \tau$ must be expressed as a function of the reconstructed $\mathrm{c} \tau$. This is accomplished by convoluting the probability density with the $\mathrm{c} \tau$ resolution function. The resolution on the reconstructed proper time $\mathrm{c} \tau_{r}$ is described by

$$
\sigma_{\mathrm{c} \tau_{r}}^{2}\left(\mathrm{c} \tau_{0}\right)=\left(\frac{m_{B}}{p_{\mathrm{t}}^{\prime}}\right)^{2} \cdot \sigma_{L_{x y}}^{2}+\left(\mathrm{c} \tau_{0}\right)^{2} \cdot \sigma_{p_{\mathrm{t}}^{\prime}}^{2}
$$

where $\mathrm{c} \tau_{0}$ is the true proper time of the event. The first term describes the contribution of the SECVTX $L_{x y}$ resolution; the second term describes the resolution of the $p_{\mathrm{t}}^{B}$ correction. Note that the second term scales with the true proper time. In the convolution of the same-sign probability density, the integral to be evaluated is

$$
P_{S S}^{\prime}\left(\mathrm{c} \tau_{r}\right)=\int_{0}^{\infty} P_{S S}\left(\mathrm{c} \tau_{0}\right) \cdot R_{\mathrm{c} \tau}\left(\mathrm{c} \tau_{0}, \mathrm{c} \tau_{r}, \sigma_{\mathrm{c} \tau_{r}}\right) \cdot d \mathrm{c} \tau_{0}
$$

where

$$
R_{\mathrm{c} \tau}\left(\mathrm{c} \tau_{0}, \mathrm{c} \tau_{r}, \sigma_{\mathrm{c} \tau_{r}}\right)=e^{-\frac{\left(\mathrm{c} \tau_{0}-\mathrm{c} \tau_{r}\right)^{2}}{2 \sigma_{\mathrm{c} \tau}}}
$$

An identical integral is evaluated for the opposite-sign probability density. If $\sigma_{\mathrm{c} \tau_{r}}$ were constant, the integrals could be done analytically, but this is clearly not the case. According to Equation $8.25, \sigma_{\mathrm{c} \tau_{r}}$ is a function of the variable of integration $\mathrm{c} \tau_{0}$.

Accordingly, this analysis will follow the procedure of many of CDF's $B$ meson lifetime measurements, such as the inclusive lepton plus charm analysis [66], which

perform the $L_{x y}$ and $p_{\mathrm{t}}^{\prime}$ integrals separately. The $L_{x y}$ convolution is an analytically simple Gaussian integral, and the $p_{\mathrm{t}}^{\prime}$ convolution is done using weighted sums from 
the $p_{\mathrm{t}}^{\prime}=p_{\mathrm{t}}^{c l} / p_{\mathrm{t}}^{B}$ distributions ${ }^{1}$ from Section 5.2.

The generic $p_{\mathrm{t}}^{c l} / p_{\mathrm{t}}^{B}$ distribution shown in Figure 8.1 will be used to illustrate the $p_{\mathrm{t}}^{\prime}$ convolution. Given an observed $M_{c l}$ and $p_{\mathrm{t}}^{c l}$ which lie in bin $i$ of $M_{c l}$ and bin $j$ of $p_{\mathrm{t}}^{c l}$, the integral over $p_{\mathrm{t}}^{\prime}$ is performed as a sum over the bins of the $i j^{\text {th }} p_{\mathrm{t}}^{c l} / p_{\mathrm{t}}^{B}$ distribution, using the value $(f(i j))$ and the relative probability $(w(i j))$ of $p_{\mathrm{t}}^{c l} / p_{\mathrm{t}}^{B}$ for each bin. Referring to the figure, $p_{\mathrm{t}}^{\prime}$, the estimate of the true $p_{\mathrm{t}}^{B}$, is given for the $\alpha^{\text {th }}$ bin by

$$
p_{\mathrm{t} \alpha}^{\prime}=p_{\mathrm{t}}^{c l} / f(i j)_{\alpha}
$$

yielding a value for the true $\mathrm{c} \tau_{0}$

$$
\mathrm{c} \tau_{0 \alpha}=\frac{L_{x y}^{0} \cdot m_{B}}{p_{\mathrm{t}_{\alpha}}^{\prime}}
$$

Mass bin i, $P_{t}$ bin $j$

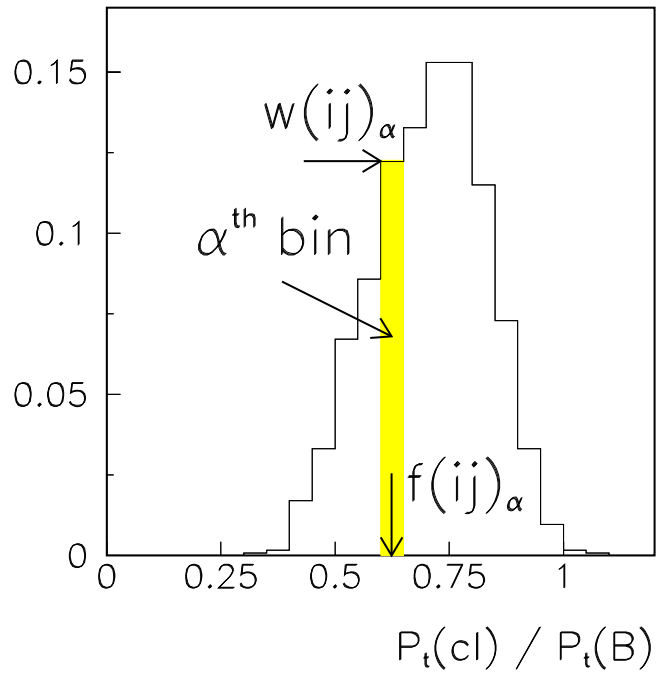

Figure 8.1: A generic $p_{\mathrm{t}}^{c l} / p_{\mathrm{t}}^{B}$ distribution.

\footnotetext{
${ }^{1}$ The $p_{\mathrm{t}}^{c l} / p_{\mathrm{t}}^{B}$ distribution is commonly called the " $\mathrm{K}$ factor" distribution.
} 
This value of $p_{\mathrm{t}}^{\prime}$ occurs with probability $w(i j)_{\alpha}$, allowing the integral of Equation 8.26 to be written as

$$
\sum_{\alpha} w(i j)_{\alpha} \cdot f(i j)_{\alpha} \cdot \int_{0}^{\infty} d L_{x y}^{0} P_{S S}\left(\mathrm{c} \tau_{0 \alpha}\right) \cdot R_{L_{x y}}\left(L_{x y}^{0}, L_{x y}^{r}, \sigma_{L_{x y}}\right)
$$

where $R_{L_{x y}}$ is a Gaussian distribution in $L_{x y}^{0}$, centered at the reconstructed decay length $L_{x y}^{r}$, of width $\sigma_{L_{x y}^{r}}$. The width is the error calculated by SECVTX. The $f(i j)_{\alpha}$ factor correctly normalizes the sum.

\subsubsection{The Total Likelihood}

The total probability densities for same- and opposite-sign events, including the $\mathrm{c} \tau$-resolution-convolution and SECVTX $\mathrm{c} \tau$ efficiencies, are

$$
\begin{aligned}
P_{S S}^{\prime}\left(L_{x y}^{r}, \sigma_{L_{x y}}, p_{\mathrm{t}}^{c l}, M_{c l}\right)= & \epsilon\left(\mathrm{c} \tau_{r}\right) \sum_{\alpha} w(i j)_{\alpha} f(i j)_{\alpha} \times \\
& \int_{0}^{\infty} d L_{x y}^{0} P_{S S}^{\prime}\left(\mathrm{c} \tau_{0} \alpha\right) R_{L_{x y}}\left(L_{x y}^{r}, L_{x y}^{0}, \sigma_{L_{x y}}\right)( \\
P_{O S}^{\prime}\left(L_{x y}^{r}, \sigma_{L_{x y}}, p_{\mathrm{t}}^{c l}, M_{c l}\right)= & \epsilon\left(\mathrm{c} \tau_{r}\right) \sum_{\alpha} w(i j)_{\alpha} f(i j)_{\alpha} \times \\
& \int_{0}^{\infty} d L_{x y}^{0} P_{O S}^{\prime}\left(\mathrm{c} \tau_{0} \alpha\right) R_{L_{x y}}\left(L_{x y}^{r}, L_{x y}^{0}, \sigma_{L_{x y}}\right)(8.3
\end{aligned}
$$

where $i, j$ again refer to each event's $p_{\mathrm{t}}^{c l}$ bin and $M_{c l}$ bin, respectively. The total negative $\log$ likelihood $-\ln \mathcal{L}$ is therefore equal to

$$
\begin{aligned}
-\ln \mathcal{L}= & -\sum_{S S} \ln P_{S S}^{\prime}\left(D_{r a w}, \frac{D_{c \bar{c}}}{D_{b \bar{b}}}, \frac{D_{\text {fake }}}{D_{b \bar{b}}}, \mathbf{F}^{\prime}, \mathbf{f}, \boldsymbol{\tau}_{\boldsymbol{H}}, P_{w s}^{s e q}\left(\mathrm{c} \tau_{r}\right), \mathrm{c} \tau_{r} \mid \Delta m_{d}, N_{D}\right) \\
& -\sum_{O S} \ln P_{O S}^{\prime}\left(D_{\text {raw }}, \frac{D_{c \bar{c}}}{D_{b \bar{b}}}, \frac{D_{\text {fake }}}{D_{b \bar{b}}}, \mathbf{F}^{\prime}, \mathbf{f}, \boldsymbol{\tau}_{\boldsymbol{H}}, P_{w s}^{s e q}\left(\mathrm{c} \tau_{r}\right), \mathrm{c} \tau_{r} \mid \Delta m_{d}, N_{D}\right)
\end{aligned}
$$


where the proper notation has been restored to illustrate the dependence of the likelihood on the fixed and free parameters. $\mathbf{F}^{\prime}$ are the $b$ hadron species fractions, f are the sample composition fractions, and $\boldsymbol{\tau}_{\boldsymbol{H}}$ the $b$ hadron lifetimes and effective

charm lifetime. The reconstructed $\mathrm{c} \tau$ depends on $L_{x y}, \sigma_{L_{x y}}, p_{\mathrm{t}}^{c l}$, and $M_{c l}$. The sums are taken over all same- and opposite-sign events. The best fit values for $\Delta m_{d}$ and $N_{D}$ are those that minimize $-\ln \mathcal{L}$.

\subsubsection{Fit Parameters}

Table 8.1 lists the fixed parameters used by the fit in the functional form of the likelihood. The $b$ hadron lifetimes and production fractions are all taken from the Particle Data Group's 1996 Review of Particle Properties [19]. The effective charm lifetime $\left(\mathrm{c} \tau_{c \bar{c}}\right)$ is not the true lifetime of charmed hadrons. While the charmed hadrons in the Monte Carlo samples are generated using the PDG charm lifetimes, $c \tau_{c \bar{c}}$ is the "lifetime" as measured in the $c \bar{c}$ Monte Carlo by the $p_{\mathrm{t}}^{B}$ correction of Section 5.2, assuming the average $b$ hadron mass.

\section{Adjustments to $b \bar{b}$ and $c \bar{c}$ Component Fractions}

The $b$ hadron species fractions listed in the table need two adjustments to match the data samples. First, the $b$ hadron species fractions in the table are the species production fractions - as all $b \bar{b}$ events enter the sample through semileptonic decay, the fraction of each species must reflect its semileptonic branching ratio. Second, the SECVTX c $\tau$ efficiency preferentially selects longer-lived hadrons over shorter-lived ones.

According to the spectator model, the partial width $\Gamma(b \rightarrow c \ell \nu)$ should be approximately the same for all $b$ hadrons [65]. Therefore the semileptonic branching ratios are proportional to the lifetimes. Explicitly, this adjustment to the $b$ hadron 


\begin{tabular}{|c|c|c|}
\hline Parameter & Value & Source \\
\hline $\mathrm{c} \tau_{B_{d}}$ & 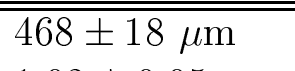 & $\bar{c} 96 \mathrm{PDG}$ \\
\hline $\mathrm{c} \tau_{B_{u}} / \mathrm{c} \tau_{B_{d}}$ & $1.02 \pm 0.05$ & '96 PDG \\
\hline $\mathrm{c} \tau_{B_{s}}$ & $483_{-27}^{+30} \mu \mathrm{m}$ & '96 PDG \\
\hline $\mathrm{c} \tau_{\Lambda_{b}}$ & $342 \pm 24 \mu \mathrm{m}$ & '96 PDG \\
\hline $\mathrm{c} \tau_{c \bar{c}}$ & $360_{-60}^{+120} \mu \mathrm{m}$ & '96 PDG; fit to $c \bar{c} \mathrm{MC}$ \\
\hline$\overline{F_{B_{u}}}$ & $37.8 \pm 2.2 \%$ & 96 PDG \\
\hline$F_{B_{d}}$ & $37.8 \pm 2.2 \%$ & ‘96 PDG \\
\hline$F_{B_{s}}$ & $11.2_{-2.6}^{+2.5 \%}$ & ‘96 PDG \\
\hline$F_{\Lambda_{b}}$ & $13.2 \pm 4.1 \%$ & '96 PDG \\
\hline $\mathrm{JC} D_{c \bar{c}} / D_{b \bar{b}}$ & $\begin{array}{l}\mathrm{e}: 0.15 \pm 0.04 \\
\mu: 0.26 \pm 0.04\end{array}$ & $b \bar{b}$ and $c \bar{c} \mathrm{MC}$ \\
\hline SLT $D_{c \bar{c}}$ & 0.5 & Assumption \\
\hline & $\begin{array}{l}\text { e: } 0.92 \pm 0.27 \\
\mu: 0.71 \pm 0.17\end{array}$ & $\begin{array}{l}\text { Fake e sample } \\
\text { Fake } \mu \text { samples }\end{array}$ \\
\hline SLT $D_{\text {fake }} / D_{b \bar{b}}$ & 0.5 & Assumption \\
\hline$f_{b \bar{b}}$ & $\begin{array}{l}\text { e: } 85.2 \pm 0.8 \% \\
\mu: 84.3 \pm 0.6 \%\end{array}$ & $p_{\mathrm{t}}^{\text {rel }}$ fit \\
\hline$f_{c \bar{c}}$ & $\begin{array}{l}\text { e: } 8.1 \pm 1.4 \% \\
\mu: 11.6 \pm 1.1 \%\end{array}$ & $p_{\mathrm{t}}^{\text {rel }}$ fit \\
\hline$f_{\text {fake }}$ & $\begin{array}{l}\text { e: } 6.7 \pm 1.5 \% \\
\mu: 4.1 \pm 1.1 \%\end{array}$ & $p_{\mathrm{t}}^{\mathrm{rel}}$ fit \\
\hline$f_{s e q}$ & $\begin{array}{l}\text { e: } 7.9 \pm 0.6 \% \\
\mu: 19.4 \pm 0.5 \%\end{array}$ & $b \bar{b} \mathrm{MC}$ \\
\hline$\Delta m_{s}$ & $700 \mathrm{ps}^{-1}$ & Assumption \\
\hline $\begin{array}{l}N_{D} \\
\Delta m_{u}\end{array}$ & Free in fit & \\
\hline$\Delta m_{d}$ & Free in fit & \\
\hline
\end{tabular}

Table 8.1: Parameters of the fit and their sources. The uncertainties are statistical only. The determination of the effective charm lifetime $\left(c \tau_{c \bar{c}}\right)$ is discussed in the text. See Section 8.2 for a discussion of the uncertainties used in the evaluation of the systematic errors. 
fractions is

$$
g_{i}=\frac{F_{i} \cdot \mathrm{c} \tau_{i}}{\sum_{i} F_{i} \cdot \mathrm{c} \tau_{i}}
$$

The sum is over the $b$ hadron production fractions $F_{B_{u}}, F_{B_{d}}, F_{B_{s}}$, and $F_{\Lambda_{b}}$, which are taken from Table 8.1. In theory, the charm hadron species fractions must be similarly adjusted. Having forced all charm hadrons to decay semileptonically in the $c \bar{c}$ event generation, however, the adjustment is already implicit in the $c \bar{c}$ samples. Thus $g=1$ for all charm events.

The probability densities assume an exponential distribution in the true proper time, which must be altered to match the SECVTX reconstructed $\mathrm{c} \tau$ efficiency. As the various hadrons have different lifetimes, they will be affected differently, thereby changing their proportions in the data. A fast Monte Carlo calculation is used to transform the SECVTX efficiency as a function of reconstructed $\mathrm{c} \tau$ to a function of true $\mathrm{c} \tau$. The parametrizations of Section 5.3 are smeared with the $\sigma_{L_{x y}}$ and $p_{\mathrm{t}}^{c l} / p_{\mathrm{t}}^{B}$ resolutions as measured from the $b \bar{b}$ Monte Carlo, thus calculating the fraction of events passing the efficiency curve as a function of the exponential lifetime. By way of example, the efficiency versus lifetime $(p f(c \tau))$ for the trigger electron sample is shown in Figure 8.2. The curve is fit to a polynomial, which is used to adjust the hadron species fractions accordingly:

$$
F_{i}^{\prime}=\frac{1}{p f\left(\mathrm{c} \tau_{i}\right)} \cdot g_{i}
$$

where in this instance, $i$ represents each $b$ hadron species as well as directly-produced charm hadrons, as charm events must pass the $\mathrm{c} \tau$ efficiency in the same manner as bottom events. The $\mathrm{c} \tau_{c \bar{c}}$ entering in Equation 8.35 is, of course, the effective charm lifetime. 


\subsubsection{Fit Results}

The fit is performed by finding the values for $\Delta m_{d}$ and $N_{D}$ that minimize the total negative log likelihood given in Section 8.1.3. To demonstrate the method, each data sample (trigger electron and muon) is fit separately, as are those events tagged by the soft lepton and jet charge flavor-taggers. The final result is taken from a combined fit over all flavor-tagged events. In the combined fit, when an event is tagged by both SLT and jet charge, the SLT flavor-tag decision and dilution is used. Also, $N_{D}$ is made into four separate free parameters, one for each combination of trigger lepton type and flavor-tag type.

The combined fit result for $\Delta m_{d}$ is $0.47 \pm 0.06 \pm 0.04$, where the first error is statistical and the second error is systematic. The systematic uncertainty is calculated in Section 8.2. The complete results for the five fits are given in Table 8.2.

\section{Fit Representation}

The results of a time-dependent mixing analysis are traditionally displayed by plotting the same-sign fraction versus $\mathrm{c} \tau_{r}$, overlaying the fit, corrected for the charm and fake backgrounds. This method is not appropriate for this analysis - it implicitly assumes that each event drives the fit with the same weight. This fit makes use of the event-by-event dilution to weight the probability density by $P_{t a g}$. Jet charge and SLT flavor-tagged events with large $\left|Q_{j e t}\right|$ or $p_{\mathrm{t}}^{\text {rel }}$ are more "important" to the fit than those with smaller $\left|Q_{j e t}\right|$ or $p_{\mathrm{t}}^{\text {rel }}$. A plot of the raw same-sign fraction would not accurately represent this important fit component, even if an appropriate result curve could be overlaid upon it.

As a correct alternative, the true same-sign fraction of $b \bar{b}$ events is plotted versus

$\mathrm{c} \tau_{r}$. For each $\mathrm{c} \tau_{r}$ bin, the number of same- and opposite-sign $b \bar{b}$ events is calculated from the sample composition fractions and the $P_{t a g}$ for each event. The particulars 


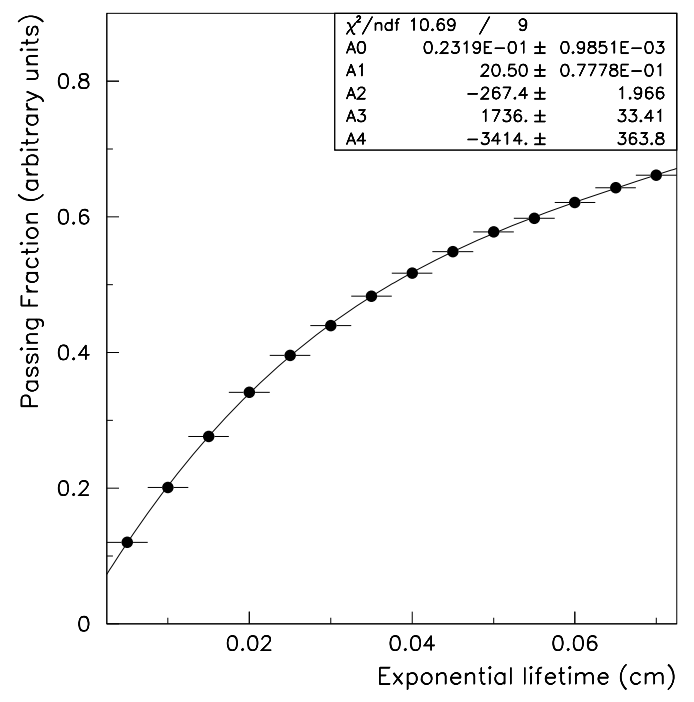

Figure 8.2: The fraction of fast Monte Carlo events passing the SECVTX efficiency as a function of the generated exponential lifetime. The data were fit to a fourth order polynomial.

\begin{tabular}{|c|c|c|c|}
\hline \multicolumn{4}{|c|}{ Soft Lepton Tagging } \\
\hline Data Set & N Events & $\Delta m_{d}\left(\mathrm{ps}^{-1}\right)$ & $N_{D}$ \\
\hline 1b ele & 3798 & $.45 \pm .07 \pm .03$ & $1.87 \pm 0.14_{-.21}^{+.20}$ \\
1b muo & 3237 & $.49 \pm .15 \pm .11$ & $1.88 \pm 0.28_{-.56}^{+.53}$ \\
\hline \hline \multicolumn{4}{|c|}{ Jet Charge Tagging } \\
\hline Data Set & N Events & $\Delta m_{d}\left(\mathrm{ps}^{-1}\right)$ & $N_{D}$ \\
\hline 1b ele & 38122 & $.40 \pm .10 \pm .03$ & $1.76 \pm 0.20_{-.23}^{+.15}$ \\
1b muo & 38819 & $.65 \pm .19 \pm .06$ & $2.60 \pm 0.45_{-.53}^{+.38}$ \\
\hline \hline \multicolumn{2}{|c|}{ Combined Soft Lepton and Jet Charge Tagging } \\
\hline Data Set & N Events & $\Delta m_{d}\left(\mathrm{ps}^{-1}\right)$ & $N_{D}$ \\
\hline 1b ele \& muo & 78118 & $.47 \pm .06 \pm .04$ & $\mathrm{SLT}(\mathrm{e}): 1.89 \pm 0.14_{-.21}^{+.19}$ \\
& & $\mathrm{SLT}(\mu): 1.82 \pm 0.21_{-.61}^{+.59}$ \\
& & $\mathrm{JC}(\mathrm{e}): 1.45 \pm 0.18_{-.21}^{+.13}$ \\
& & $\mathrm{JC}(\mu): 1.77 \pm 0.34_{-.35}^{+.26}$ \\
\hline
\end{tabular}

Table 8.2: $\Delta m_{d}$ and $N_{D}$ fit results for the individual flavor-taggers and trigger lepton samples as well as a combined fit. The systematic uncertainties are taken from Section 8.2. In the combined fit, if an event is tagged by both flavor-taggers, the decision and dilution of the soft lepton tag is used. 
of the calculation are shown in Appendix B. The fit result is represented by the output of an FMC $b \bar{b}$ simulation with $\Delta m_{d}$ and $N_{D}$ set to the values returned by the fit. The simulation includes the correct $\mathrm{c} \tau_{r}$ resolution for a proper comparison to the data representation.

Figure 8.3 shows $f_{S S}(b \bar{b})$ versus $\mathrm{c} \tau_{r}$ for the four indivdual fits. The points are the data. The error bars include the statistical error only, and not the uncertainty on $N_{D}$ as reported by the fit. The dashed histogram is the FMC fit representation. The representation of the final combined fit is shown in Figure 8.4.

\subsubsection{Fit Algorithm Check}

To assure the integrity of the maximum-likelihood fit algorithm and software, an FMC is used to create simulated samples using resolution functions and data samplederived parameters. Thousands of samples with the same statistics and dilutions of the data samples are generated and fit. The returned values for $\Delta m_{d}$ and $N_{D}$ can be compared to the input values for any bias from the fitting procedure and the scale of the reported statistical error checked as well.

By way of example, the fit results from a set of FMC experiments representing the SLT-tagged trigger electron sample is shown in Figure 8.5. The fit algorithm clearly returns the input values. The figure also shows the distribution of the reported errors on the parameters, and the normalized fit residuals $\left(\Delta m_{d}^{f i t}-\right.$ $\left.\Delta m_{d}^{\text {input }}\right) / \sigma$ and $\left(N_{D}^{f i t}-N_{D}^{\text {input }}\right) / \sigma$. The widths of the normalized residuals are approximately one for both $\Delta m_{d}$ and $N_{D}$, demonstrating that the statistical error is calculated properly and the probability density is correctly normalized. Figure 8.6 shows the distribution of the negative log likelihoods from the FMC fits. The negative log likelihood from the fit to the data sample is indicated on the plot. It lies well within the distribution, providing further evidence for the reliability of the 
fitting procedure. Similar studies for the other individual fits and the combined fit establish confidence in each case.
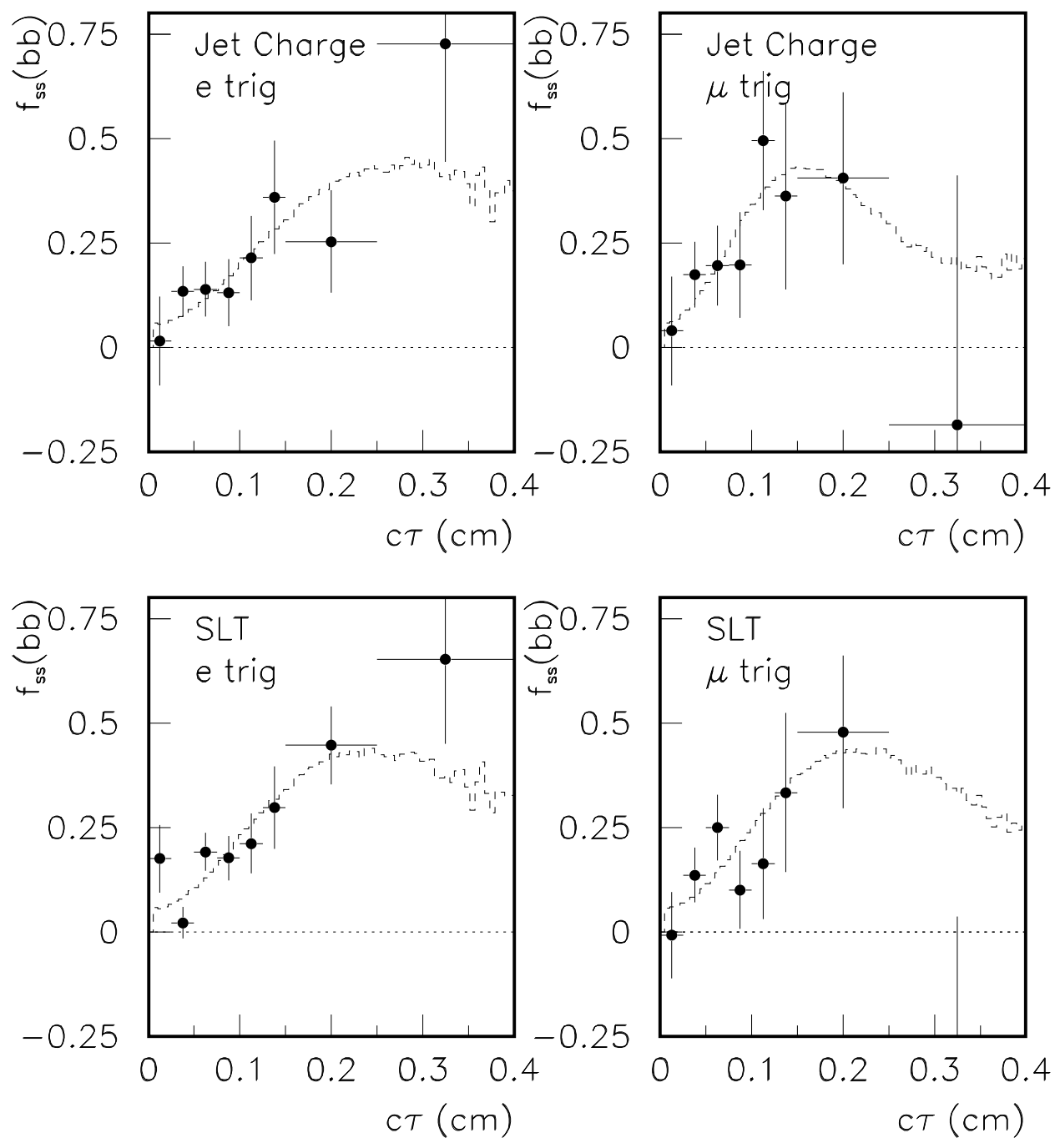

Figure 8.3: The $b \bar{b}$ same-sign fraction $f_{S S}(b \bar{b})$ versus $\mathrm{c} \tau_{r}$ for the individual flavor-taggers and trigger lepton samples. The points represent the data and the dashed histogram the fit result. 


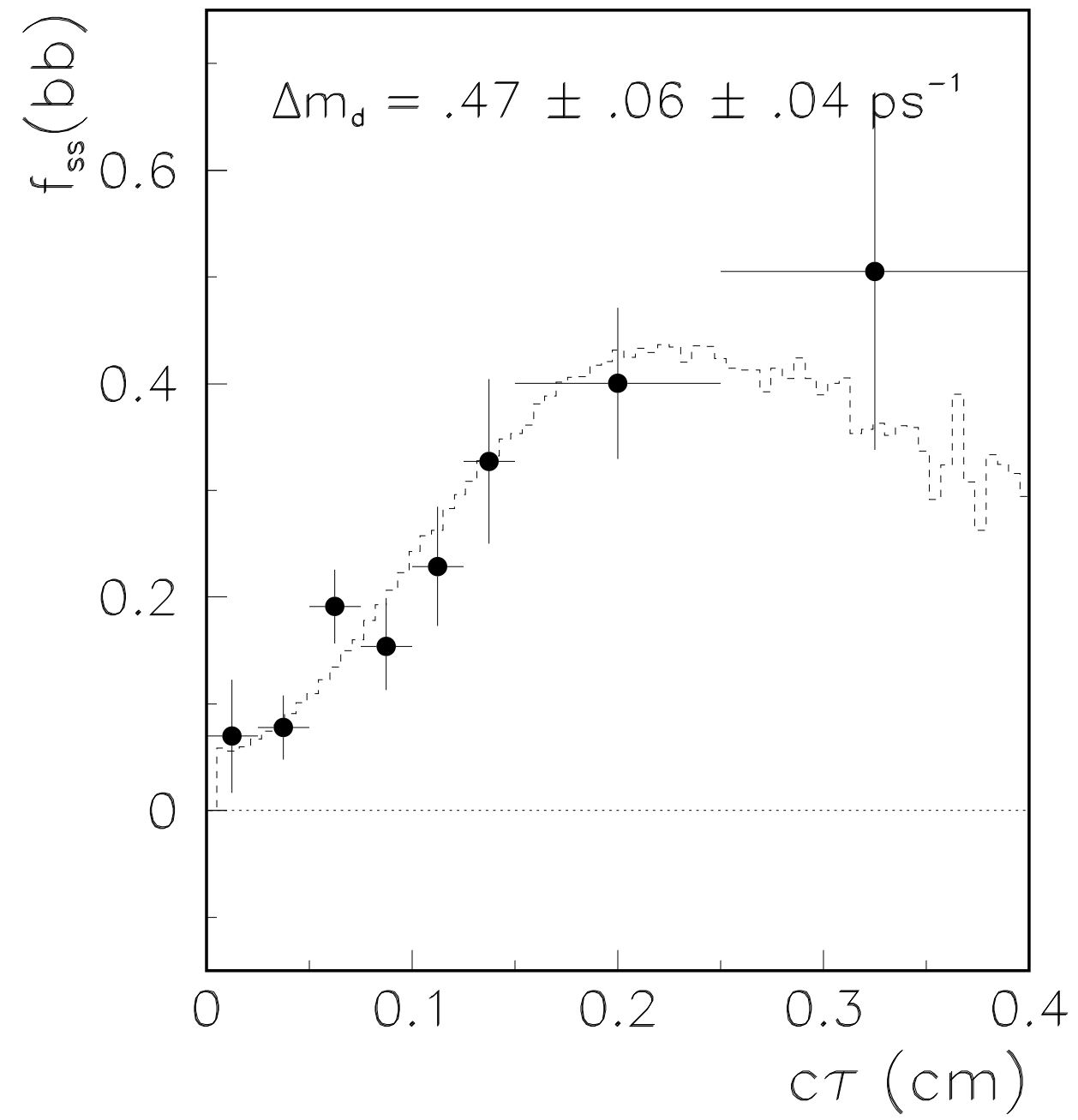

Figure 8.4: The $b \bar{b}$ same-sign fraction $f_{S S}(b \bar{b})$ versus $\mathrm{c} \tau_{r}$ for the combined fit. The points represent the data and the dashed histogram the fit result. 


\section{SLT Electron Trigger FMC Fits}
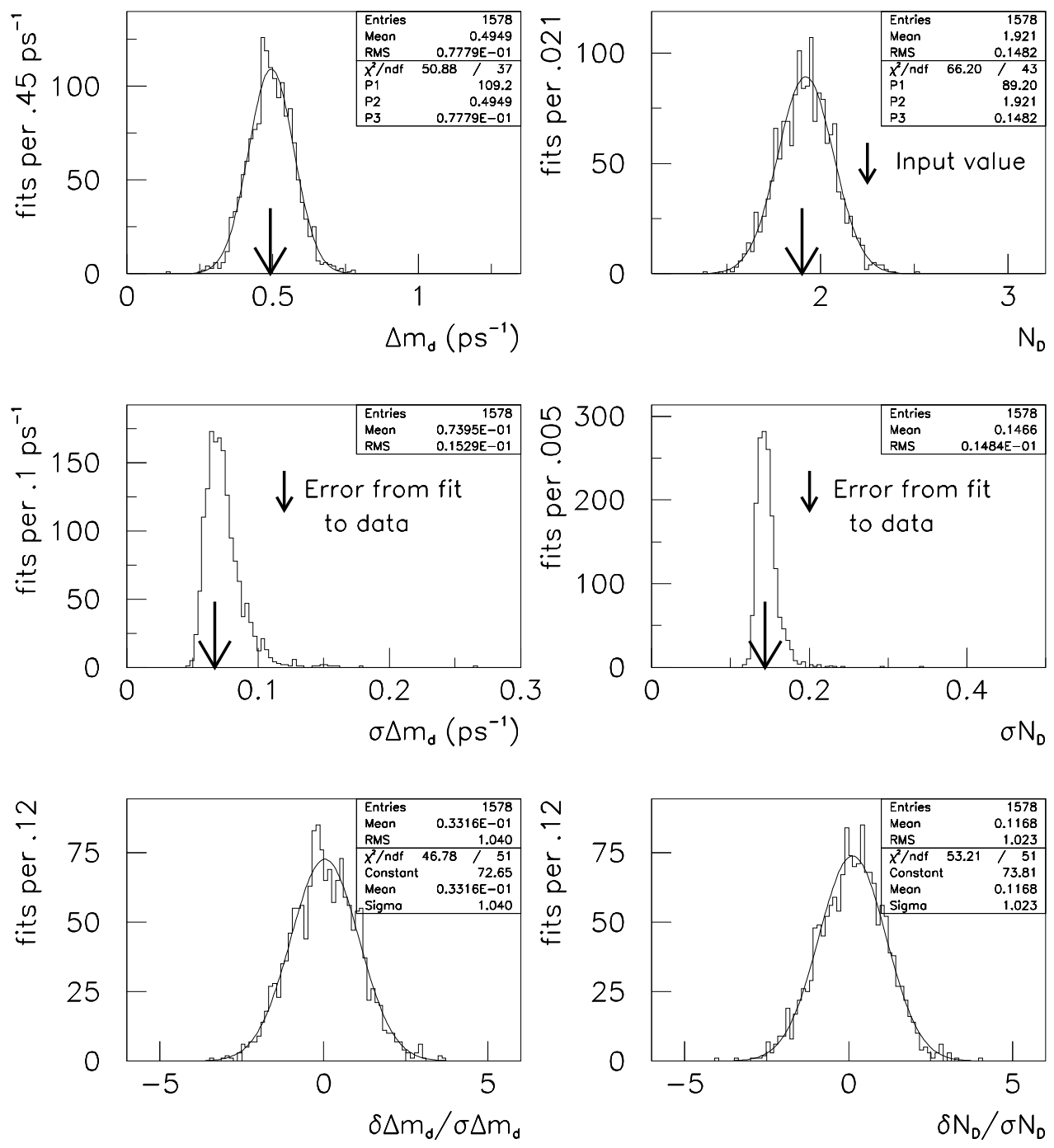

Figure 8.5: Fast Monte Carlo fits representing the SLT flavor-tagged electron trigger sample.

Top left and right: The spectrum of fitted values for $\Delta m_{d}$ and $N_{D}$. The arrows represent the input values for the FMC.

Middle left and right: The spectrum of fit-returned errors on the fit parameters. The arrows represent the errors from the fit to the SLT flavor-tagged trigger electron sample. Bottom left and right: The distribution of $\delta / \sigma$ for the fit parameters, where $\delta=$ (fit-input). The distributions are fit to a Gaussian; the widths are approximately one, indicating that the error scale is correct. 


\section{SLT Electron Trigger Toy MC Fits}

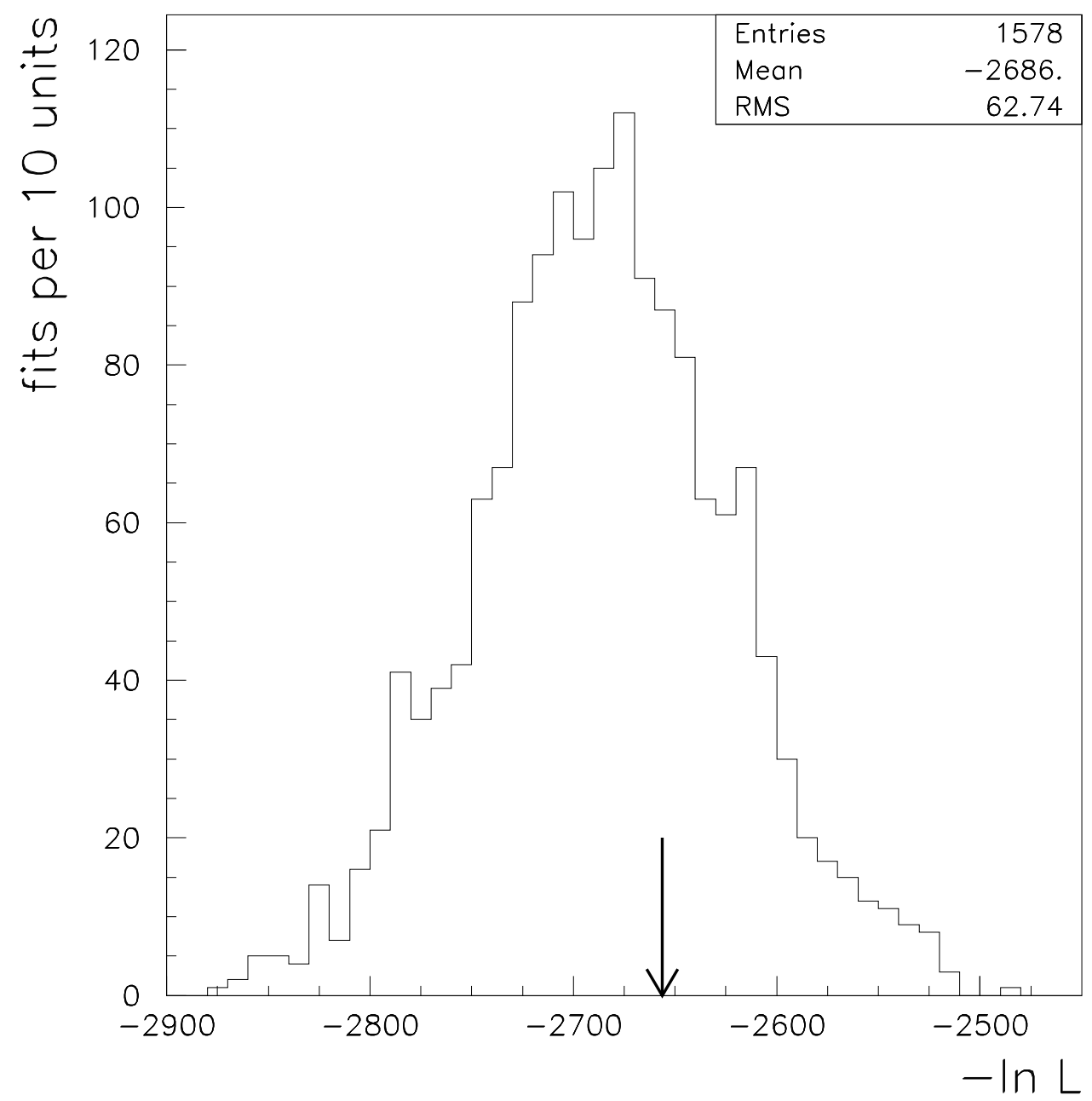

Figure 8.6: Negative log likelihood values for fits of the FMC SLT flavor-tagged electron trigger samples. The arrow indicates the negative log likelihood value of the fit of the data sample. 


\subsection{Systematic Uncertainties}

Tables 8.3, 8.4, 8.5, and 8.6 summarize the systematic errors for the individual flavor-taggers in the electron and muon trigger data sets separately. Table 8.7 summarizes the systematic errors for the combined fit with both flavor-taggers and both samples.

- SLT $D_{c \bar{c}}$ : The soft lepton $D_{c \bar{c}}$ must be varied over the entire allowable range (from 0 to 1 ), in the absence of a reliable estimate from either Monte Carlo or data. This is the largest single contribution to the systematic uncertainty on $\Delta m_{d}$.

- JC $D_{c \bar{c}} / D_{b \bar{b}}$ : Though the jet charge $D_{c \bar{c}} / D_{b \bar{b}}$ is determined from Monte Carlo, the ratio is conservatively varied over the entire allowable range (from 0 to 1$)$.

- $D_{\text {fake }} / D_{b \bar{b}}$ : As the fake leptons are believed to be from $b \bar{b}$ decay products, the upper limit on $D_{\text {fake }}$ should be $D_{b \bar{b}}$. The ratio is conservatively varied from 0 to 1 , but the contribution to the total systematic uncertainty is still insignificant.

- $\left\langle\delta L_{x y}\right\rangle$ : The observed decay length is adjusted by an average bias $\left\langle\delta L_{x y}\right\rangle$ derived from the $b \bar{b}$ Monte Carlo. The adjustment is varied from no bias to twice the nominal value. This is the second most significant contribution to the systematic uncertainty.

- $\sigma_{L_{x y}}$ : To test the effect of the SECVTX-reported uncertainty on $L_{x y}$, it is scaled by $\pm 20 \%$.

- $\epsilon(\mathrm{c} \tau)$ : The SECVTX $\mathrm{c} \tau$ efficiency is adjusted as discussed in Section 5.3 
and shown in Figures 5.4 and 5.5. This is the fourth largest contribution to the systematic uncertainty.

- $f_{\bar{c} \bar{c}}$ and $f_{f a k e}$ : The fractions are varied by $3 \%$. The size of the variation is taken from the difference between the $p_{\mathrm{t}}^{\text {rel }}$ and $M_{c l}$ methods for determining the sample composition.

- $\mathrm{c} \tau_{B_{d}^{0}}, \mathrm{c} \tau_{B_{s}^{0}}, \mathrm{c} \tau_{\Lambda_{b}}$, and $\mathrm{c} \tau_{B_{u}^{-}} / \mathrm{c} \tau_{B_{d}^{0}}$ : These are varied by their quoted uncertainties from the 1996 Review of Particle Properties [19]. The uncertainty on $\mathrm{c} \tau_{B_{u}^{-}} / \mathrm{c} \tau_{B_{d}^{0}}$ gives the third largest contribution to the systematic uncertainty.

- $\mathrm{c} \tau_{c \bar{c}}$ : The effective charm lifetime is varied by $\pm 60 \mu \mathrm{m}$. This large variation is due to the $c \bar{c}$ Monte Carlo sample having been generated with forced semileptonic charm hadron decays, which alters the $D^{+}: D^{0}$ ratio. The size of the variation covers any bias on $c \tau_{c \bar{c}}$.

- $f_{B_{s}^{0}}$ and $f_{\Lambda_{b}}$ : These are varied individually by their quoted errors from the 1996 RPP [19], absorbing the variation in the other $b$ hadron fractions, while keeping the ratios between those fractions constant.

- $f_{\text {seq }}$ : To assure no bias from the $b \bar{b}$ generation, $f_{\text {seq }}$ is varied by $\pm 25 \%$ of its value, keeping the $\mathrm{c} \tau_{r}$ shape fixed.

- $\Delta m_{s}$ : The default value for $\Delta m_{s}$ is assumed to be very large, resulting in half the $B_{s}^{0} \mathrm{~s}$ mixing, independent of $\mathrm{c} \tau . \Delta m_{s}$ is lowered to $7 \mathrm{ps}^{-1}$, which is below the current measured lower limit [40]. The contribution to the total systematic uncertainty is insignificant. 


\begin{tabular}{|l|l||r|r||r|r|}
\hline \multicolumn{5}{|c|}{ SLT Flavor-tag, Trigger Electron Sample } \\
\hline Parameter & Variation & \multicolumn{2}{|c|}{$\delta \Delta m_{d}\left(\mathrm{ps}^{-1}\right)$} & \multicolumn{2}{|c|}{$\delta N_{D}$} \\
\hline \hline SLT, $e: D_{c \bar{c}}$ & {$[0,1]$} & -0.018 & +0.020 & +0.17 & -0.18 \\
\hline SLT, $e: D_{f a k e} / D_{b \bar{b}}$ & {$[0,1]$} & +0.000 & +0.000 & +0.04 & -0.04 \\
\hline$e:\left\langle\delta L_{x y}\right\rangle$ & {$[0, \times 2]$} & +0.021 & -0.009 & +0.01 & -0.00 \\
\hline$\sigma_{L_{x y}}$ & $\pm 20 \%$ & +0.001 & -0.001 & -0.00 & +0.00 \\
\hline$\epsilon(\mathrm{c} \tau)$ & & -0.010 & -0.001 & -0.02 & -0.01 \\
\hline$e: f_{c \bar{c}}$ & $\pm 3.0 \%$ & +0.008 & -0.008 & -0.02 & +0.01 \\
\hline$e: f_{f a k e}$ & $\pm 3.0 \%$ & -0.000 & +0.000 & +0.02 & -0.02 \\
\hline $\mathrm{c} \tau_{B_{u}} / \mathrm{c} \tau_{B_{d}}$ & $1.02 \pm 0.05$ & +0.010 & -0.012 & -0.00 & +0.00 \\
\hline $\mathrm{c} \tau_{B_{d}}$ & $468 \pm 18 \mu \mathrm{m}$ & -0.002 & +0.003 & -0.01 & +0.01 \\
\hline $\mathrm{c} \tau_{B_{s}}$ & $483 \pm 30 \mu \mathrm{m}$ & -0.004 & +0.004 & +0.00 & -0.01 \\
\hline $\mathrm{c} \tau_{\Lambda_{b}}$ & $\pm 24 \mu \mathrm{m}$ & +0.003 & -0.003 & -0.00 & +0.00 \\
\hline $\mathrm{c} \tau_{c \bar{c}}$ & $423 \pm 60 \mu \mathrm{m}$ & +0.005 & -0.006 & +0.01 & -0.01 \\
\hline$f_{B_{s}}$ & $\left(11.1_{-2.6}^{+2.5}\right) \%$ & -0.001 & +0.001 & +0.05 & -0.05 \\
\hline$f_{\Lambda_{b}}$ & $(13.2 \pm 4.1) \%$ & +0.005 & -0.005 & -0.01 & +0.01 \\
\hline$e: f_{\text {seq }}$ & $\pm 25 \%$ & +0.002 & -0.002 & +0.07 & -0.06 \\
\hline$\Delta m_{s}$ & $\rightarrow 7 \mathrm{ps}^{-1}$ & \multicolumn{2}{|c||}{+0.001} & +0.00 \\
\hline \hline \hline Total Systematics & & $+.033\left(\mathrm{ps}^{-1}\right)$ & $+\mathbf{0 . 2 0}$ \\
\hline
\end{tabular}

Table 8.3: Systematic uncertainties for the $\Delta m_{d}$ and $N_{D}$ fit using the soft lepton flavortag in the trigger electron sample. 


\begin{tabular}{|l|l||l|l||c|c|}
\hline \multicolumn{5}{|c|}{ SLT Flavor-tag, Trigger Muons } \\
\hline Parameter & Variation & \multicolumn{2}{|c||}{$\delta \Delta m_{d}\left(\mathrm{ps}^{-1}\right)$} & \multicolumn{2}{c|}{$\delta N_{D}$} \\
\hline \hline SLT, $\mu: D_{c \bar{c}}$ & {$[0,1]$} & -0.082 & +0.105 & +0.48 & -0.52 \\
\hline SLT, $\mu: D_{\text {fake }} / D_{b \bar{b}}$ & {$[0,1]$} & -0.000 & -0.000 & -0.00 & -0.00 \\
\hline$\mu:\left\langle\delta L_{x y}\right\rangle$ & {$[0, \times 2]$} & -0.001 & -0.042 & -0.06 & -0.00 \\
\hline$\sigma_{L_{x y}}$ & $\pm 20 \%$ & +0.002 & -0.002 & +0.00 & -0.00 \\
\hline$\epsilon(\mathrm{c} \tau)$ & & -0.015 & -0.008 & -0.04 & -0.01 \\
\hline$\mu: f_{c \bar{c}}$ & $\pm 3.0 \%$ & +0.026 & -0.025 & -0.06 & +0.05 \\
\hline$\mu: f_{\text {fake }}$ & $\pm 3.0 \%$ & +0.000 & +0.000 & -0.00 & -0.00 \\
\hline $\mathrm{c} \tau_{B_{u}} / \mathrm{c} \tau_{B_{d}}$ & $1.02 \pm 0.05$ & +0.016 & -0.018 & +0.01 & -0.01 \\
\hline $\mathrm{c} \tau_{B_{d}}$ & $468 \pm 18 \mu \mathrm{m}$ & -0.006 & +0.007 & -0.01 & +0.01 \\
\hline $\mathrm{c} \tau_{B_{s}}$ & $483 \pm 30 \mu \mathrm{m}$ & -0.007 & +0.006 & +0.00 & -0.00 \\
\hline $\mathrm{c} \tau_{\Lambda_{b}}$ & $\pm 24 \mu \mathrm{m}$ & +0.005 & -0.005 & +0.00 & -0.00 \\
\hline $\mathrm{c} \tau_{c \bar{c}}$ & $423 \pm 60 \mu \mathrm{m}$ & +0.022 & -0.035 & +0.03 & -0.05 \\
\hline$f_{B_{s}}$ & $\left(11.1_{-2.6}^{+2.5} \%\right.$ & -0.001 & +0.001 & +0.05 & -0.05 \\
\hline$f_{\Lambda_{b}}$ & $(13.2 \pm 4.1) \%$ & +0.010 & -0.010 & -0.01 & +0.01 \\
\hline$\mu: f_{\text {seq }}$ & $\pm 25 \%$ & -0.003 & +0.002 & +0.21 & -0.17 \\
\hline$\Delta m_{s}$ & $\rightarrow 7 \mathrm{ps}^{-1}$ & -0.002 & -0.01 \\
\hline \hline \hline Total Systematics & & \multicolumn{2}{|c|}{$+\mathbf{1 1 3}\left(\mathrm{ps}^{-1}\right)$} & $+\mathbf{0 . 5 3}$ \\
& & -.107 & $\left(\mathrm{ps}^{-1}\right)$ & $-\mathbf{0 . 5 6}$ \\
\hline
\end{tabular}

Table 8.4: Systematic uncertainties for the $\Delta m_{d}$ and $N_{D}$ fit using the soft lepton flavortag in the trigger muon sample. 


\begin{tabular}{|l|l||l|l||r|r|}
\hline \multicolumn{5}{|c|}{ Jet Charge Flavor-tag, Trigger Electrons } \\
\hline Parameter & Variation & \multicolumn{2}{|c|}{$\delta \Delta m_{d}\left(\mathrm{ps}^{-1}\right)$} & \multicolumn{2}{|c|}{$\delta N_{D}$} \\
\hline \hline $\mathrm{JC}, e: D_{c \bar{c}} / D_{b \bar{b}}$ & {$[0,1]$} & -0.004 & +0.025 & +0.04 & -0.20 \\
\hline $\mathrm{JC}, e: D_{f a k e} / D_{b \bar{b}}$ & {$[0,1]$} & +0.000 & +0.000 & +0.10 & -0.01 \\
\hline$e:\left\langle\delta L_{x y}\right\rangle$ & {$[0, \times 2]$} & +0.001 & -0.018 & -0.04 & -0.00 \\
\hline$\sigma_{L_{x y}}$ & $\pm 20 \%$ & +0.000 & +0.000 & -0.00 & +0.00 \\
\hline$\epsilon(\mathrm{c} \tau)$ & & -0.010 & -0.005 & -0.02 & -0.01 \\
\hline$e: f_{c \bar{c}}$ & $\pm 3.0 \%$ & +0.003 & -0.003 & +0.06 & -0.06 \\
\hline$e: f_{f a k e}$ & $\pm 3.0 \%$ & -0.000 & +0.000 & +0.00 & +0.00 \\
\hline $\mathrm{c} \tau_{B_{u}} / \mathrm{c} \tau_{B_{d}}$ & $1.02 \pm 0.05$ & +0.017 & -0.017 & +0.01 & -0.01 \\
\hline $\mathrm{c} \tau_{B_{d}}$ & $468 \pm 18 \mu \mathrm{m}$ & +0.000 & +0.000 & -0.00 & +0.00 \\
\hline $\mathrm{c} \tau_{B_{s}}$ & $483 \pm 30 \mu \mathrm{m}$ & -0.004 & +0.004 & +0.01 & -0.01 \\
\hline $\mathrm{c} \tau_{\Lambda_{b}}$ & $\pm 24 \mu \mathrm{m}$ & +0.004 & -0.004 & +0.00 & +0.00 \\
\hline $\mathrm{c} \tau_{c \bar{c}}$ & $423 \pm 60 \mu \mathrm{m}$ & -0.005 & +0.007 & -0.01 & +0.01 \\
\hline$f_{B_{s}}$ & $\left(11.1_{-2.6}^{+2.5}\right) \%$ & -0.000 & +0.001 & +0.05 & -0.05 \\
\hline$f_{\Lambda_{b}}$ & $(13.2 \pm 4.1) \%$ & +0.003 & -0.003 & -0.01 & +0.01 \\
\hline$e: f_{s e q}$ & $\pm 25 \%$ & +0.002 & -0.001 & +0.06 & -0.06 \\
\hline$\Delta m_{s}$ & $\rightarrow 7 \mathrm{ps}$ & \multicolumn{2}{|c||}{-0.003} & -0.01 \\
\hline \hline Total Systematics & $+.032(\mathrm{ps}$ & \\
\hline
\end{tabular}

Table 8.5: Systematic uncertainties for the $\Delta m_{d}$ and $N_{D}$ fit using the jet charge flavor-tag in the trigger electron sample. 


\begin{tabular}{|l|l||r|r|||c|c|}
\hline \multicolumn{5}{|c|}{ Jet Charge Flavor-tag, Trigger Muon Sample } \\
\hline Parameter & Variation & \multicolumn{2}{|c|}{$\delta \Delta m_{d}\left(\mathrm{ps}^{-1}\right)$} & \multicolumn{2}{|c|}{$\delta N_{D}$} \\
\hline \hline $\mathrm{JC}, \mu: D_{c \bar{c}} / D_{b \bar{b}}$ & {$[0,1]$} & -0.023 & +0.038 & +0.20 & -0.46 \\
\hline $\mathrm{JC}, \mu: D_{f a k e} / D_{b \bar{b}}$ & {$[0,1]$} & +0.001 & -0.000 & +0.11 & -0.04 \\
\hline$\mu:\left\langle\delta L_{x y}\right\rangle$ & {$[0, \times 2]$} & +0.043 & -0.033 & +0.08 & -0.00 \\
\hline$\sigma_{L_{x y}}$ & $\pm 20 \%$ & +0.005 & -0.004 & +0.01 & -0.01 \\
\hline$\epsilon(\mathrm{c} \tau)$ & & +0.008 & -0.002 & +0.04 & -0.01 \\
\hline$\mu: f_{c \bar{c}}$ & $\pm 3.0 \%$ & +0.007 & -0.007 & +0.06 & -0.06 \\
\hline$\mu: f_{\text {fake }}$ & $\pm 3.0 \%$ & -0.001 & +0.001 & +0.02 & -0.02 \\
\hline $\mathrm{c} \tau_{B_{u}} / \mathrm{c} \tau_{B_{d}}$ & $1.02 \pm 0.05$ & +0.006 & -0.008 & -0.01 & +0.01 \\
\hline $\mathrm{c} \tau_{B_{d}}$ & $468 \pm 18 \mu \mathrm{m}$ & -0.003 & +0.003 & +0.00 & -0.00 \\
\hline $\mathrm{c} \tau_{B_{s}}$ & $483 \pm 30 \mu \mathrm{m}$ & -0.004 & +0.004 & +0.00 & -0.00 \\
\hline $\mathrm{c} \tau_{\Lambda_{b}}$ & $\pm 24 \mu \mathrm{m}$ & +0.005 & -0.005 & -0.00 & +0.00 \\
\hline $\mathrm{c} \tau_{c \bar{c}}$ & $423 \pm 60 \mu \mathrm{m}$ & -0.007 & +0.007 & -0.02 & +0.03 \\
\hline$f_{B_{s}}$ & $\left(11.1_{-2.6}^{+2.5}\right) \%$ & +0.000 & +0.000 & +0.07 & -0.07 \\
\hline$f_{\Lambda_{b}}$ & $(13.2 \pm 4.1) \%$ & +0.010 & -0.009 & -0.02 & +0.02 \\
\hline$\mu: f_{\text {seq }}$ & $\pm 25 \%$ & +0.002 & -0.002 & +0.28 & -0.23 \\
\hline$\Delta m_{s}$ & $\rightarrow 7 \mathrm{ps}^{-1}$ & \multicolumn{2}{|c||}{-0.005} & -0.02 \\
\hline \hline Total Systematics & & +.061 & $\left(\mathrm{ps}^{-1}\right)$ & $+\mathbf{0 . 3 8}$ \\
\hline
\end{tabular}

Table 8.6: Systematic uncertainties for the $\Delta m_{d}$ and $N_{D}$ fit using the jet charge flavor-tag in the trigger muon sample. 


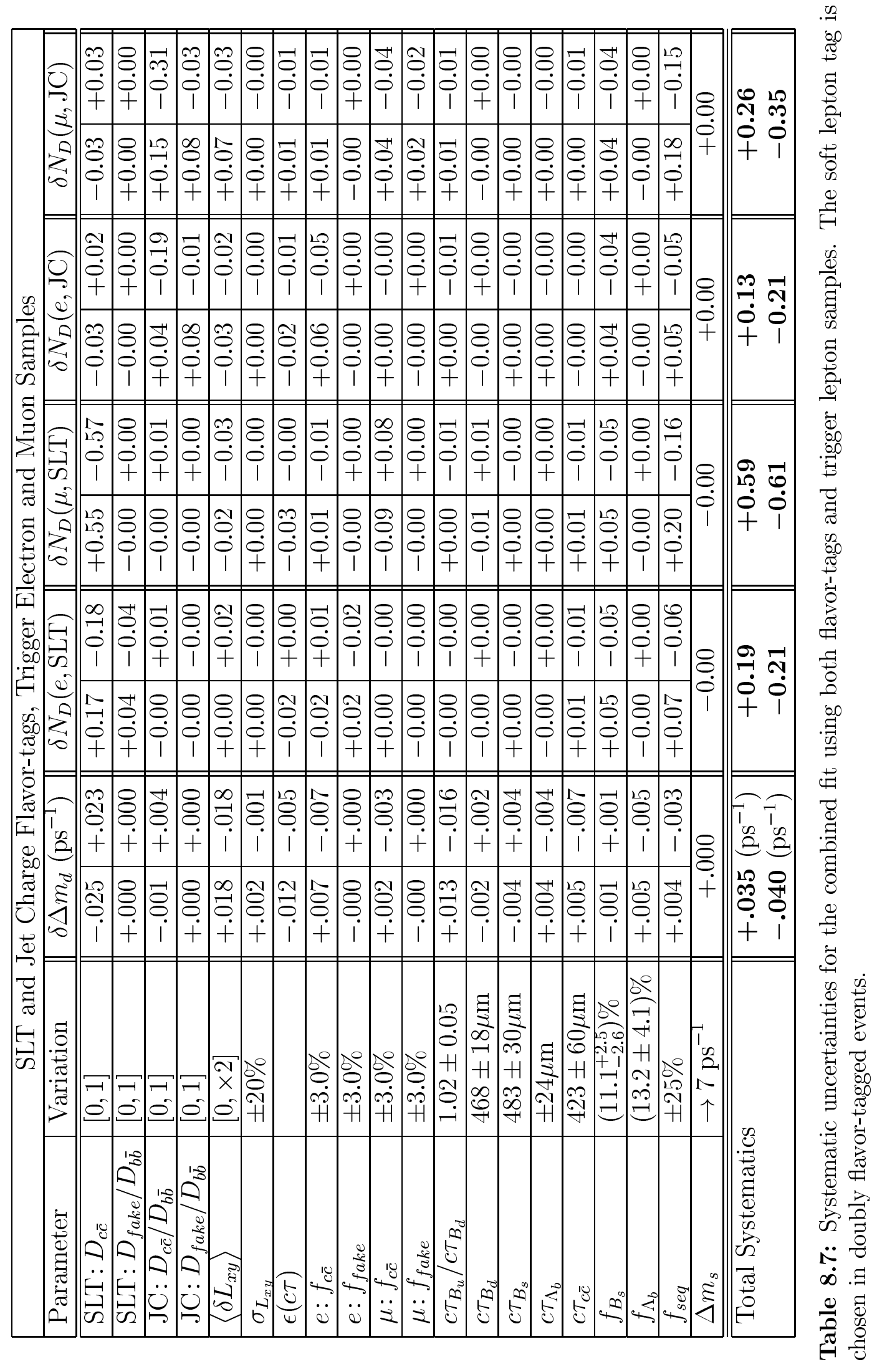




\section{Chapter 9}

\section{Conclusions}

The mass difference $\Delta m_{d}=M\left(B_{H}^{0}\right)-M\left(B_{L}^{0}\right)$ has been measured from the time dependence of $B_{d}^{0}-\bar{B}_{d}^{0}$ oscillations. Using both soft lepton and jet charge flavortagging in CDF's Run 1B inclusive electron and muon datasets, the combined fit yields $\Delta m_{d}=0.47 \pm 0.06 \pm 0.04 \mathrm{ps}^{-1}$, where the first error is statistical and the second error is systematic. This corresponds to a value of $x_{d} \equiv \frac{\Delta m_{d}}{\Gamma_{B_{d}^{0}}}=0.73 \pm 0.10 \pm 0.07$, where $\Gamma_{B_{d}^{0}}=0.64 \pm 0.02 \mathrm{ps}^{-1}$. The result is in excellent agreement with the LEP experiments' average $\Delta m_{d}=0.466 \pm 0.019$ [40].

The measurement of $\Delta m_{d}$ contributes to a measurement of $\left|V_{t d} V_{t b}^{*}\right|^{2}$. Assuming $\left|V_{t b}\right|=1$, Equation 1.18 can be re-written as

$$
\left|V_{t d}\right|^{2}=\frac{6 \pi^{2} \Delta m_{d}}{G_{F}^{2} m_{B_{d}^{0}} f_{B_{d}^{0}}^{2} B_{B_{d}^{0}} m_{W}^{2} S\left(\frac{m_{t}}{m_{W}}\right) \eta_{Q C D}}
$$

By using the result of this analysis and the values for the other parameters given in Section 1.3.1, one finds

$$
\left|V_{t d}\right|=(8.5 \pm 1.7) \times 10^{-3}
$$


The uncertainty on $\left|V_{t d}\right|$ is completely dominated by the uncertainty on $f_{B_{d}^{0}} B_{B_{d}^{0}}^{1 / 2}$.

This analysis also serves as an evaluation of the future prospects of CDF's $b$ physics program, since the same experimental techniques can be used in measurements of $B_{s}^{0}$ mixing and $C P$-violation. Both the Tevatron and the CDF detector are currently undergoing major upgrades. Run 2, scheduled to begin in 1999, is expected to deliver an integrated luminosity of $2 \mathrm{fb}^{-1}$. The CDF detector upgrades [42], including a new silicon vertex detector (SVX II) and expanded muon coverage, will improve both the soft lepton and jet charge flavor-tagging algorithms and the $b \bar{b}$ event selection. Making a projection [67] from the expected amount of data, the detector upgrade capabilities, and the effectiveness of flavor-tagging demonstrated by this analysis, measurements of $C P$-violation will soon be within CDF's reach. 


\section{Bibliography}

[1] E. Rutherford, Phil. Mag. 21, 669 (1911).

[2] T.D. Lee and C.N. Yang, Phys. Rev. 104, 254 (1956).

[3] C.S. Wu et. al., "Experimental Test of Parity Conservation in Beta Decay", Phys. Rev. 105, 1413 (1957); R.L. Garwin, L.M. Lederman, and M. Weinrich, Phys. Rev. 105, 1415 (1957).

[4] H. Frauenfelder et. al., "Parity and the Polarization of Electrons from Co ${ }^{60 ",}$ Phys. Rev. 106, 386 (1957).

[5] M. Goldhaber, L. Grodzins, and A.W. Sunyar, "Helicity of Neutrinos", Phys. Rev. 109, 1015 (1958).

[6] J.H. Christenson, J.W. Cronin, V.L. Fitch, and R. Turlay, "Evidence for the $2 \pi$ Decay of the $K_{2}^{0}$ Meson", Phys. Rev. Lett. 13, 138 (1964).

[7] S.L. Glashow, Nucl. Phys. 22, 579 (1961); S. Weinberg, Phys. Rev. Lett. 19, 1264 (1967); A. Salam, Elementary Particle Theory: Relativistic Groups and Analyticity (Nobel Symposium No. 8), edited by W. Svartholm (Almqvist and Wiksell, Stockholm 1968), p. 367.

[8] P.W. Higgs, Phys. Rev. Lett. 12, 132 (1964); Phys. Rev. 145, 1156 (1966);

G. 't Hooft, Nucl. Phys. B33, 167 (1971); Nucl. Phys. B35, 167 (1971).

[9] H. Fritzsch, M. Gell-Mann, and H. Leutwyler, Phys. Lett. B47, 365 (1973).

[10] B.T. Cleveland et. al., Nucl. Phys. (Proc. Supp.) B38, 47 (1995); P. Anselmann et. al., Phys. Lett. B327, 377 (1994).

[11] N. Cabibbo, Phys. Rev. Lett. 10, 531 (1963).

[12] M. Kobayashi and K. Maskawa, Progr. Theor. Phys. 49, 652 (1973).

[13] J.J. Aubert et. al., "Experimental Observation of a Heavy Particle J", Phys. Rev. Lett. 33, 1404 (1974); J.-E. Augustin et. al., "Discovery of a Narrow Resonance in $\mathrm{e}^{+} \mathrm{e}^{-}$Annihilation", Phys. Rev. Lett. 33, 1406 (1974). 
[14] S.L. Glashow, J. Iliopoulos, and L. Maiani, Phys. Rev. D2, 1285 (1970).

[15] T. Akagi et. al., "Experimental Study of the Rare Decays $K_{L}^{0} \rightarrow \mu e, K_{L}^{0} \rightarrow e e$, $K_{L}^{0} \rightarrow \mu \mu, K_{L}^{0} \rightarrow$ eeee", Phys. Rev. D51, 2061 (1995).

[16] G. Goldhaber et. al., "Observation in $e^{+} e^{-}$Annihilation of a Narrow State at $1865 \mathrm{MeV} / \mathrm{c}^{2}$ Decaying to $K \pi$ and $K \pi \pi \pi$ ", Phys. Rev. Lett. 37, 256 (1976).

[17] L. Wolfenstein, Phys. Rev. Lett. 51, 1945 (1983).

[18] A. Ali, " $B^{0}$ Anti- $B^{0}$ Mixings and Rare $B$ Decays", a lecture given at the 26th International Conference on High Energy Physics (ICHEP 92), published in High Energy Physics (proceedings), edited by J.R. Sanford (American Institute of Physics 1993).

[19] R.M. Barnett et. al., "Review of Particle Properties", Phys. Rev. D54, 1 (1996).

[20] M.L. Perl et. al., Phys. Rev. Lett. 35, 1489 (1975).

[21] S.W. Herb et. al., Phys. Rev. Lett. 39, 252 (1977).

[22] PLUTO Collaboration, Ch. Berger et. al., Phys. Lett 76B, 243 (1978); C.W. Darden et. al., Phys. Lett. 76B, 246 (1978); Phys. Lett. 78B, 364 (1978).

[23] D. Andrews et. al., Phys. Rev. Lett. 44, 219 (1980); G. Finocchiaro et. al., Phys. Rev. Lett. 45, 222 (1980).

[24] P. Nason, S. Dawson, and R.K. Ellis, Nucl. Phys. B303, 608 (1988); B327, 49 (1989).

[25] A. Martin, R. Roberts, and J. Stirling, "Parton distributions of the proton", Phys. Rev. D50, 6734 (1994).

[26] C. Peterson et. al., Phys. Rev. D27, 105 (1983); J. Chrin, Z. Phys. C36, 163 (1983).

[27] C.S. Kim and A.D. Martin, Phys. Lett. 225B, 186 (1989).

[28] K. Lande et. al., "Observation of Long-Lived Neutral V Particles", Phys. Rev. 103, 1901 (1956).

[29] A.J. Buras, W. Slominski, and H. Steger, Nucl. Phys. B245, 369 (1984).

[30] J.M. Flynn, "Developments in Lattice QCD", Plenary talk at the 28th International Conference on High Energy Physics (ICHEP 96), published in High Energy Physics (proceedings), edited by Z. Ajduk and A.K. Wroblewski (World Scientific 1997). 
[31] T. Inami and C.S. Lim, Prog. Theor. Phys. 65, 297 (1981); A.J. Buras, Nucl. Instrum. Meth. A368, 1 (1995).

[32] A.J. Buras, Nucl. Phys. B347, 491 (1990).

[33] M. Dine, "Baryon Number Violation in the Standard Model", lectures given at the Theoretical Advanced Study Institute (TASI 92), published in Recent Directions in Particle Theory: From Superstrings and Black Holes to the Standard Model (proceedings), edited by J. Harvey and J. Polchinski (World Scientific 1993).

[34] ARGUS Collaboration, H. Albrecht et. al., "Observation of $B^{0} \bar{B}^{0}$ Mixing", Phys. Lett. B192, 245 (1987).

[35] ALEPH Collaboration, D. Buskulic et. al., "Improved Measurement of the $B_{d}^{0}$ $\bar{B}_{d}^{0}$ Oscillation Frequency", Preprint CERN-PPE-96-102, July 1996. Submitted to Z. Phys. C.

[36] OPAL Collaboration, G. Alexander et. al., "A Measurement of the $B_{d}^{0}$ Oscillation Frequency Using Leptons and $D^{* \pm}$ Mesons", Z. Phys. C72, 377 (1996).

[37] DELPHI Collaboration, P. Abreu et. al., "Measurement of the $B_{d}^{0}$ Oscillation Frequency Using Kaons, Leptons and Jet Charge", Z. Phys. C72, 17 (1996).

[38] L3 Collaboration, M. Acciarri et. al., "Measurement of the $B_{d}^{0}$ Oscillation Frequency", Phys. Lett. B383, 487 (1996).

[39] UA1 Collaboration, C. Albajar et. al., "Measurement of $B^{0}-\bar{B}^{0}$ Mixing at the CERN Sp̄̄S Collider", Phys. Lett. B262, 171 (1991).

[40] R. Forty, " $V_{c b}, V_{t d} / V_{t s}$ Review (LEP, CLEO and CDF)", a lecture given at the 2nd International Conference on $B$ Physics and $C P$ Violation (1997), to be published by World Scientific.

[41] CDF Collaboration, F. Abe et. al., "The CDF Detector: An Overview", Nucl. Instrum. Meth. A271, 387 (1988).

[42] CDF-II Collaboration, R. Blair et. al., "The CDF II Detector: Technical Design Report", FERMILAB-PUB-96/390-E (1996).

[43] H. Minemura et. al., "Construction and Testing of a 3-m Diameter $\times 5-\mathrm{m}$ Superconducting Solenoid for the Fermilab Collider Detector Facility (CDF)", Nucl. Instrum. Meth. A238, 18 (1985).

[44] C. Newman-Holmes, E. E. Schmidt, and R. Yamada, "Measurement of the Magnetic Field of the CDF Magnet", Nucl. Instrum. Meth. A274, 443 (1989). 
[45] F. Bedeschi et. al., "Design and Construction of the CDF Central Tracking Chamber", Nucl. Instrum. Meth. A268, 50 (1988).

[46] P. Azzi et. al., "SVX': The New CDF Silicon Vertex Detector", Nucl. Instrum. Meth. A360, 137 (1995).

[47] D. Amidei et. al., "The Silicon Vertex Detector of the Collider Detector at Fermilab", Nucl. Instrum. Meth. A350, 73 (1994).

[48] CDF Collaboration, F. Abe et. al., "The Topology of Three Jet Events in $p \bar{p}$ Collisions at $\sqrt{s}=1.8$ TeV", Phys. Rev. D45, 1448 (1992).

[49] G. Ascoli et. al., "CDF Central Muon Detector", Nucl. Instrum. Meth. A268, 33 (1988).

[50] CDF Collaboration, F. Abe et. al., "Inclusive Jet Cross-section in $p \bar{p}$ Collisions at $\sqrt{s}=1.8$ TeV", Phys. Rev. Lett. 77, 438 (1996).

[51] CDF Collaboration, F. Abe et. al., "Further Properties of High-mass Multi-jet Events at the Fermilab $p \bar{p}$ Collider", Phys. Rev. D54, 4221 (1996).

[52] A.B. Wicklund, private communication.

[53] W. Yao, F. Bedeschi, P. Berge, L. Galtieri, D. Gerdes, J. Incandela, F. Tartarelli, G. Unal, with the B-Tag Group, "A Seed Vertexing b-Tag Algorithm for Top," CDF Note 2716 (1994).

[54] CDF Collaboration, F. Abe et. al., "Evidence for Top Quark Production in $p \bar{p}$ Collisions at $\sqrt{s}=1.8$ TeV", Phys. Rev. D50, 2966 (1994); summarized in Phys. Rev. Lett. 73, 225 (1994).

[55] CDF Collaboration, F. Abe et. al., "Observation of Top Quark Production in $p \bar{p}$ Collisions with CDF Detector at Fermilab," Phys. Rev. Lett. 74, 2626 (1995).

[56] S. Dell'Angelo et. al., "A Primary Vertex Finding Package," CDF Note 1789 (1992); S. Dell'Angelo, F. Bedeschi, "Combining the Primary Vertex Finder with the SVX Beam Line Measurement," CDF Note 2055 (1993); F. Bedeschi, S. Dell'Angelo, "Handling Multiple Primary Vertices with the Primary Vertex Finder," CDF Note 2084 (1993).

[57] H. Wenzel, D. Benjamin, and A. Sill, "Beamlines," CDF Note 3334 (1995); H. Wenzel, "Fitting the Beamline with the SVX," CDF Note 1924 (1993).

[58] R. Mattingly and P. Sphicas, "A Measurement of the Correlated $b \bar{b}$ Cross Section," CDF Note 1930 (1993). 
[59] T. Sjostrand, "High-energy Physics Event Generation with PYTHIA 5.7 and JETSET 7.4", Comput. Phys. Commun. 82, 74 (1994); T. Sjostrand, "PYTHIA 5.6 and JETSET 7.3: Physics and Manual", Preprint CERN-TH6488-92 (May 1992).

[60] P. Avery, K. Read, and G. Trahern, "QQ: a Monte Carlo Generator", CLEO Software Note CSN-212 (1985); J.D. Lewis and P. Avery, "CLEOMC: The CDF Interface to the CLEO Monte Carlo (QQ)", CDF Note 2724 (1994).

[61] H. Y. Chao et. al., "The SVX' Monte Carlo Simulation", CDF Note 2946 (1995).

[62] M. Paulini, private communication.

[63] D. Kestenbaum, "Observation of Top Quark Anti-top Quark Production Using a Soft Lepton $b$ tag in Proton Anti-proton Collisions at 1.8 TeV", Ph.D. Thesis, Harvard University, UMI-96-31521 (1996).

[64] OPAL Collaboration, R. Akers et. al., "Measurement of the Time Dependence of $B_{d}^{0}-\bar{B}_{d}^{0}$ Mixing Using a Jet Charge Technique", Phys. Lett. B327, 411 (1994).

[65] M. Neubert and C.T. Sachrajda, "Spectator Effects in Inclusive Decays of Beauty Hadrons", Nucl. Phys. B483, 339 (1997).

[66] CDF Collaboration, F. Abe et. al., "Measurement of the $B$ and $\bar{B}$ Meson Lifetimes Using Semileptonic Decays", Phys. Rev. Lett. 76, 4462 (1996).

[67] M. Paulini, "Heavy Flavor Physics from Top to Bottom", a lecture given at the 24th Annual SLAC Summer Institute on Particle Physics: The Strong Interaction, From Hadrons to Protons (SSI 96), FERMILAB-CONF-96/456-E (1996). 


\section{Appendix A}

\section{Dilution and "Effective Tagging Efficiency"}

Consider a mixing asymmetry measurement. Let $N$ be the number of events in the sample, $N_{u}$ be the number of events with no mixing, and $N_{m}$ be the number where mixing has occurred. The true mixing asymmetry is

$$
A_{\text {true }}=\frac{N_{u}-N_{m}}{N_{u}+N_{m}}
$$

Due to imperfections in the flavor-tagging technique, the measured asymmetry $\left(A_{\text {meas }}\right)$ will be different from $A_{\text {true }}$. Suppose that, in the sample, $N_{O S}+N_{S S}$ events are flavor-tagged, of which $N_{O S}$ are tagged as unmixed and $N_{S S}$ as mixed. The tagging efficiency is $\epsilon=\left(N_{O S}+N_{S S}\right) / N$. The measured asymmetry is

$$
A_{\text {meas }}=\frac{N_{O S}-N_{S S}}{N_{O S}+N_{S S}}
$$

Because the tagging technique is imperfect, the tagged events consist of events tagged correctly as well as incorrectly. Therefore, given the probability of correctly 
tagging the $b$ flavor $\left(P_{t a g}\right)$, and the probability of incorrectly tagging the $b$ flavor $\left(P_{\text {mistag }}\right)$, the number of events tagged as unmixed is

$$
N_{O S}=\epsilon P_{t a g} N_{u}+\epsilon P_{m i s t a g} N_{m}
$$

and the number tagged as mixed is

$$
N_{S S}=\epsilon P_{\text {tag }} N_{m}-\epsilon P_{\text {mistag }} N_{u}
$$

Note that $P_{\text {mistag }}=1-P_{\text {tag }}$. Also, the flavor-tagging dilution $(D)$ is equal to $P_{t a g}-P_{\text {mistag }}$ (see Section 7.1). The measured asymmetry is

$$
\begin{aligned}
A_{\text {meas }} & =\frac{N_{O S}-N_{S S}}{N_{O S}+N_{S S}} \\
& =\frac{\epsilon P_{\text {tag }} N_{u}+\epsilon P_{\text {mistag }} N_{m}-\epsilon P_{\text {tag }} N_{m}-\epsilon P_{\text {mistag }} N_{u}}{\epsilon P_{\text {tag }} N_{u}+\epsilon P_{\text {mistag }} N_{m}+\epsilon P_{\text {tag }} N_{m}+\epsilon P_{\text {mistag }} N_{u}} \\
& =\frac{\left(P_{\text {tag }}-P_{\text {mistag }}\right)\left(N_{u}-N_{m}\right)}{\left(P_{\text {tag }}+P_{\text {mistag }}\right)\left(N_{u}+N_{m}\right)} \\
& =D \cdot \frac{N_{u}-N_{m}}{N_{u}+N_{m}} \\
& =D A_{\text {true }}
\end{aligned}
$$

An implicit assumption has been made that the ability of the flavor-tagger to yield a correct decision is independent of whether it sees a mixed or unmixed state. While $A_{\text {true }}$ measured with an imperfect tagger does not depend on the tagging efficiency, its uncertainty does.

The expression for the uncertainty, given in Equation 7.5, is derived accordingly. Assuming Poisson-distributed errors on $N_{O S}$ and $N_{S S}$, the expression for the 
variance on $A_{\text {meas }}$ is given by

$$
\begin{aligned}
\sigma_{A_{\text {meas }}}^{2} & =N_{O S}\left[\frac{\partial}{\partial N_{O S}} A_{\text {meas }}\right]^{2}+N_{S S}\left[\frac{\partial}{\partial N_{S S}} A_{\text {meas }}\right]^{2} \\
& =\frac{4 N_{O S} N_{S S}^{2}+4 N_{O S}^{2} N_{S S}}{\left(N_{O S}+N_{S S}\right)^{4}} \\
& =\frac{4 N_{O S} N_{S S}\left(N_{O S}+N_{S S}\right)}{\left(N_{O S}+N_{S S}\right)^{4}} \\
& =\frac{\left(N_{O S}^{2}+N_{S S}\right)^{2}-\left(N_{O S}^{2}-N_{S S}\right)^{2}}{\left(N_{O S}+N_{S S}\right)^{3}} \\
& =\frac{1-A_{\text {meas }}^{2}}{N_{O S}+N_{S S}}
\end{aligned}
$$

As $A_{\text {true }}=A_{\text {meas }} / D$ and $N_{O S}+N_{S S}=N \epsilon$, Equation A.6 becomes

$$
\begin{aligned}
\sigma_{A_{\text {true }}}^{2} & =\frac{1}{D^{2}} \cdot \sigma_{A_{\text {meas }}}^{2} \\
& =\frac{1}{D^{2}} \cdot \frac{1-A_{\text {meas }}^{2}}{N_{O S}+N_{S S}} \\
& =\frac{1-D^{2} A_{\text {true }}^{2}}{N \epsilon D^{2}}
\end{aligned}
$$

To achieve the smallest uncertainty on $\Delta m_{d}, \epsilon D^{2}$ must be maximized. There are three flavor-tagging methods used in this analysis (SLT e, SLT $\mu$, and jet charge), and the task is to combine them in the way that gives the largest $\epsilon D^{2}$. Two such methods will be described here. The derivations to follow are made more straightforward by making the assumption that the three flavor-tagging methods used in this analysis (SLT e, SLT $\mu$, and jet charge) never overlap (that is, no event is tagged by more than one tagger). 


\section{Method 1: Average Dilution}

The simplest way to combine the flavor-tagging results is to take the set of all opposite- and same-sign events from the taggers and calculate an average dilution. This method weights the decisions from each tagger equally. The average dilution is

$$
D_{a v g}=\frac{\sum_{i=1}^{3}\left(N_{O S}^{i}-N_{S S}^{i}\right)}{\sum_{i=1}^{3}\left(N_{O S}^{i}+N_{S S}^{i}\right)}
$$

This expression is equivalent to

$$
D_{a v g}=\frac{\sum_{i=1}^{3} \epsilon_{i} D_{i}}{\sum_{i=1}^{3} \epsilon_{i}}
$$

Equation A.9 makes it clear that the average dilution $D_{a v g}$ is the weighted average of the individual flavor-tagging dilutions. The efficiencies are the weights. The total $\epsilon D^{2}$ from this scheme is

$$
\begin{aligned}
\epsilon D^{2} & =\left(\sum_{i=1}^{3} \epsilon_{i}\right) \cdot D_{a v g}^{2} \\
& =\left(\sum_{i=1}^{3} \epsilon_{i}\right) \cdot\left[\frac{\sum_{i=1}^{3} \epsilon_{i} D_{i}}{\sum_{i=1}^{3} \epsilon_{i}}\right]^{2} \\
& =\frac{\left[\sum_{i=1}^{3} \epsilon_{i} D_{i}\right]^{2}}{\sum_{i=1}^{3} \epsilon_{i}}
\end{aligned}
$$




\section{Method 2: Averaging Measurements}

Another way to combine the flavor-taggers is to use each to make an independent

measurement of $A_{\text {true }}$, and combine the measurements in the familiar error-weighted average:

$$
\begin{aligned}
A_{\text {true }} & =\frac{\sum_{i=1}^{3} \frac{A_{\text {true }}^{i}}{\sigma_{i}^{2}}}{\sum_{i=1}^{3} \frac{1}{\sigma_{i}^{2}}} \\
& =\frac{\sum_{i=1}^{3} \frac{A^{i} N \epsilon_{i} D_{i}}{1-D_{i}^{2} A_{i}^{2}}}{\sum_{i=1}^{3} \frac{N \epsilon_{i} D_{i}^{2}}{1-D_{i}^{2} A_{i}^{2}}}
\end{aligned}
$$

The uncertainty of the combined measurement is

$$
\begin{aligned}
\frac{1}{\sigma_{A_{\text {true }}}^{2}} & =\sum_{i=1}^{3} \frac{1}{\sigma_{i}^{2}}=\sum_{i=1}^{3} \frac{N \epsilon_{i} D_{i}^{2}}{1-D_{i}^{2} A_{i}^{2}} \\
\lim _{D_{i} A_{i} \ll 1} \sigma_{A_{\text {true }}}^{2} & \rightarrow \frac{1}{\sum_{i=1}^{3} N \epsilon_{i} D_{i}^{2}}
\end{aligned}
$$

The limit of small $D A$ makes it clear that the total effective tagging efficiency for the combination is

$$
\epsilon D^{2}=\sum_{i=1}^{3} \epsilon_{i} D_{i}^{2}
$$




\section{Maximizing $\epsilon D^{2}$}

Which of the two methods yields the largest $\epsilon D^{2}$ ? It is the second. The proof is obtained by using a formalism from elementary statistics. The variance of a weighted distribution must be non-negative, for non-negative weights. Calculating the variance of the dilution of the three flavor-taggers, using the efficiencies as the weights, yields

$$
\begin{aligned}
\operatorname{Var}(D) & \equiv\left\langle D^{2}\right\rangle-\langle D\rangle^{2} \geq 0 \\
\left\langle D^{2}\right\rangle & \geq\langle D\rangle^{2} \\
\frac{\sum \epsilon_{i} D_{i}^{2}}{\sum \epsilon_{i}} & \geq\left[\frac{\sum \epsilon_{i} D_{i}}{\sum \epsilon_{i}}\right]^{2} \\
\sum \epsilon_{i} D_{i}^{2} & \geq \frac{\left[\sum \epsilon_{i} D_{i}\right]^{2}}{\sum \epsilon_{i}}
\end{aligned}
$$

The left-hand side of Equation A.15 is the total $\epsilon D^{2}$ from Equation A.14, the right-hand side that of Equation A.10. Weighting the flavor-taggers equally can never result in a larger $\epsilon D^{2}$ than combining the separate measurements of the mixing asymmetry from each tagger. The proof can be supported by the following argument. The jet charge flavor-tagger has a very large efficiency, but only a moderate dilution; the SLT flavor-tagger has a much smaller efficiency, but a large dilution. If all tags are grouped together, then the SLT dilution is "lost" in the jet charge dilution. The average dilution from all tags together will give an $\epsilon D^{2}$ which is less than the $\epsilon D^{2}$ from SLT flavor-tags alone.

Equation A.15 offers the motivation for parametrizing the jet charge and SLT flavor-tagging dilution versus $\left|Q_{j e t}\right|$ and $p_{\mathrm{t}}^{\mathrm{rel}}$, respectively. Consider the soft electron flavor-tags: Section 7.2.4 demonstrates that those tag electrons with large $p_{\mathrm{t}}^{\text {rel }}$ yield a larger dilution than those with small $p_{\mathrm{t}}^{\mathrm{rel}}$. If all tag electrons in the trigger electron 
sample were taken together, the average dilution would be $22.3 \%$ (there are 912 opposite-sign and 579 same-sign tag electrons in 89,262 events). As the efficiency is $1.67 \%$, the total $\epsilon D^{2}$ would be $0.08 \%$. But by dividing the flavor-tags in bins of $p_{\mathrm{t}}^{\mathrm{rel}}$, an $\epsilon D^{2}$ of $0.13 \%$ is obtained (see Table 7.6). By separating the high-dilution tags from the low-dilution tags, just as is done between the jet charge and the SLT flavor-tags, the total $\epsilon D^{2}$ is increased.

The argument for using an event-by-event dilution in the maximum-likelihood fit of Chapter 8 is as follows. The action of separating the flavor-tags into an ever-larger number of bins to maximize $\epsilon D^{2}$ can be taken to the extreme. Each flavor-tagged event allows, by itself, a measurement of $\Delta m_{d}$, albeit one with very poor statistics. But by the central limit theorem, the average of all the measurements from each event converges to the correct value for $\Delta m_{d}$. From Equation A.14, each flavortagged event will contribute $D_{i}^{2}=\left(2 \cdot P_{t a g}^{i}-1\right)^{2}$ to the total $\epsilon D^{2}$, with efficiency $1 / N$. Thus the total $\epsilon D^{2}$ from this method is

$$
\epsilon D^{2}=\frac{1}{N} \sum_{i=1}^{n} D_{i}^{2}
$$

demonstrating how Equation 7.6 follows from Equation A.14. 


\section{Appendix B}

\section{Determination of $f_{S S}(b \bar{b})$}

The same-sign fraction of $b \bar{b}$ events $\left(f_{S S}(b \bar{b})\right)$ versus reconstructed proper time $\mathrm{c} \tau_{r}$ must be determined with a maximum-likelihood fit. The estimated dilution $D_{i}$, the same- or opposite-sign assignment, and the sample compositions are inputs to the fit. For the $i^{t h}$ event in the $j^{t h} \mathrm{c} \tau_{r}$ bin, the likelihood functions $\mathcal{L}_{S S}^{i j}$ and $\mathcal{L}_{O S}^{i j}$ are given by

$$
\begin{aligned}
\mathcal{L}_{S S}^{i j}= & f_{b \bar{b}}^{j} \cdot\left(1-P_{\text {seq }}^{j}\right) \cdot \frac{1}{2}\left(1-D_{i} \cdot\left(1-2 f_{S S}(b \bar{b})^{j}\right)\right) \\
& +f_{b \bar{b}}^{j} \cdot P_{\text {seq }}^{j} \cdot \frac{1}{2}\left(1+D_{i} \cdot\left(1-2 f_{S S}(b \bar{b})^{j}\right)\right) \\
& +f_{\text {fake }}^{j} \cdot\left(1-P_{\text {seq }}^{j}\right) \cdot \frac{1}{2}\left(1-\frac{D_{\text {fake }}}{D_{b \bar{b}}} \cdot D_{i} \cdot\left(1-2 f_{S S}(b \bar{b})^{j}\right)\right) \\
& +f_{\text {fake }}^{j} \cdot P_{\text {seq }}^{j} \cdot \frac{1}{2}\left(1+\frac{D_{\text {fake }}}{D_{b \bar{b}}} \cdot D_{i} \cdot\left(1-2 f_{S S}(b \bar{b})^{j}\right)\right) \\
& +f_{c \bar{c}}^{j} \cdot \frac{1}{2}\left(1-D_{i} \cdot \frac{D_{c \bar{c}}}{D_{b \bar{b}}}\right) \\
\mathcal{L}_{O S}^{i j}= & f_{b \bar{b}}^{j} \cdot\left(1-P_{\text {seq }}^{j}\right) \cdot \frac{1}{2}\left(1+D_{i} \cdot\left(1-2 f_{S S}(b \bar{b})^{j}\right)\right) \\
& +f_{b \bar{b}}^{j} \cdot P_{\text {seq }}^{j} \cdot \frac{1}{2}\left(1-D_{i} \cdot\left(1-2 f_{S S}(b \bar{b})^{j}\right)\right) \\
& +f_{\text {fake }}^{j} \cdot\left(1-P_{\text {seq }}^{j}\right) \cdot \frac{1}{2}\left(1+\frac{D_{\text {fake }}}{D_{b \bar{b}}} \cdot D_{i} \cdot\left(1-2 f_{S S}(b \bar{b})^{j}\right)\right)
\end{aligned}
$$




$$
\begin{aligned}
& +f_{\text {fake }}^{j} \cdot P_{s e q}^{j} \cdot \frac{1}{2}\left(1-\frac{D_{\text {fake }}}{D_{b \bar{b}}} \cdot D_{i} \cdot\left(1-2 f_{S S}(b \bar{b})^{j}\right)\right) \\
& +f_{c \bar{c}}^{j} \cdot \frac{1}{2}\left(1+D_{i} \cdot \frac{D_{c \bar{c}}}{D_{b \bar{b}}}\right)
\end{aligned}
$$

$D_{i} \cdot \frac{D_{c \bar{c}}}{D_{b \bar{b}}}$ is replaced by 0.5 for SLT flavor-tagged events, as described in Section 8.1.1. The $\mathrm{c} \tau_{r}$ dependence of $f_{b \bar{b}}+f_{\text {fake }}$ relative to $f_{c \bar{c}}$ for each $\mathrm{c} \tau_{r}$ bin is determined with a fast Monte Carlo. The overall negative $\log$ likelihood for each $\mathrm{c} \tau_{r}$ bin is given by

$$
-\ln \mathcal{L}_{j}=-\sum_{i}^{S S} \ln \mathcal{L}_{S S}^{i j}-\sum_{k}^{O S} \ln \mathcal{L}_{O S}^{k j}
$$

with $f_{S S}(b \bar{b})^{j}$ being the only free parameter in the fit. The appropriate value of $f_{S S}(b \bar{b})^{j}$ for the $j^{\text {th }} \mathrm{c} \tau$ bin is that which minimizes $-\ln \mathcal{L}_{j}$. 


\section{Appendix C}

\section{$d E / d x$ Corrections}

It is possible to distinguish between particle species using the energy loss $(d E / d x)$ in matter. The $d E / d x$ method relies on the fact that the most probable value of the ionization energy loss is a function of the particle's $\beta \gamma$ rather than its momentum. A simultaneous measurement of momentum and $d E / d x$ therefore determines the particle's mass.

$d E / d x$ can be an important tool in many particle physics analyses. Though the resolution of CDF's Run 1 CTC $d E / d x$ system is poor, the $d E / d x$ information can still be used in this analysis to reduce backgrounds for the soft electron tagger.

While developing this analysis, it became apparent that there were serious problems with the software designed to provide corrections to the $d E / d x$ measurements in the CTC. Specifically, the software mishandled the data from the last one-third of Run 1B, during which approximately one-half of the Run 1B data were taken. The impact on the soft electron tagger was to pass too many tracks, sharply reducing the electron-hadron discrimination. The nature of the problems is not germane to this analysis, and will not be described here. This Appendix is concerned only with the derivation of the new $d E / d x$ corrections and prediction curves of the response 
versus $\beta \gamma$ from the data.

\section{C.1 A CDF $d E / d x$ Primer}

At CDF, the CTC $d E / d x$ information is stored as the digital pulse width between the leading edge and the trailing edge times for each CTC hit in superlayers 3 through 8 ; it is expressed in units of nanoseconds. The two elements of a track's $d E / d x$ are QCTC, the $80 \%$ truncated mean of each unshared wire's pulse width ${ }^{1}$, and NCTC, the number of wires used to form the mean, after truncation. Wires "shared" by more than one track are ignored. Both QCTC and NCTC are calculated for every CTC track by production, and stored in the event record. QCTC must be corrected for many effects to be a reliable estimator of a particle's $d E / d x$. For clarity, the truncated mean before any corrections will be referred to as UNQCTC. QCTC is reserved for the corrected $d E / d x$ response.

The largest correction is for the track's path-length (the local distance traversed near a CTC wire). The total charge collected by the wire will obviously be a function of this distance. The desired quantity is charge per unit length. For momenta above $3 \mathrm{GeV} / \mathrm{c}$, the contribution to the path-length is in the r-z plane, and therefore is proportional to $1 / \sin (\theta)$. For this reason, the path-length is referred to as $d \ell d r$. The path-length correction must also include a contribution from wire-gain saturation, which occurs near an angle of $\theta=90^{\circ}$, and is parametrized as a function of both $d \ell d r$ and $\cos \theta$. An adjustment for NCTC is added, as the $80 \%$ truncated mean produces a bias, as is a contribution from the instantaneous luminosity.

What complicates the correction procedure is that each of these corrections in

\footnotetext{
${ }^{1}$ The probability distribution of a particle's energy losses in matter has a Landau distribution. The $20 \%$ of wires with the highest width are not used to calculate the mean $d E / d x$, so that the mean is insensitive to the high tail of the Landau.
} 
turn depend on the aspect angle: the angle between the particle's trajectory and the drift direction in a CTC cell. For high- $p_{\mathrm{t}}$ tracks, the aspect angle is very close to $90^{\circ}$; softer tracks will turn away from the radial direction and have an aspect angle farther from $90^{\circ}$. Thus the cosine of the angle has an inverse relationship with the $p_{\mathrm{t}}$, and the same sign as the charge of the track. In this Appendix, "aspect angle" will refer to the cosine of the angle, and is abbreviated as aspc. In addition to the corrections mentioned above, the aspect angle affects a track's $d E / d x$ directly. Each correction is parametrized as a function of $a s p c$, along with the direct contribution.

\section{C.2 First Correction Studies With Conversions}

Electrons are an ideal choice for mapping the performance of the $d E / d x$ system. They are present in the data in abundance, from photon conversions in the material of the detector. By virtue of their low mass, their mean $d E / d x$ is virtually flat as a function of momentum. The $d E / d x$ corrections are therefore those that make the measured electron mean $d E / d x$ response constant under all circumstances.

The correction procedure is lengthy. The goal is to obtain a multi-dimensional parametrization of the mean UNQCTC depending on all the variables requiring correction. This is done by considering the variables one at a time, at each step

fitting the shape of the mean UNQCTC minus the current parametrization using a maximum-likelihood fitter. Throughout this Appendix, "residual" refers to the difference between a track's UNQCTC and the parametrization. When the shape of the mean residuals versus the new variable is determined, the full UNQCTC distribution is refit allowing all parameters to vary, which allows the fit to account for any correlations between them. The UNQCTC parametrization includes a constant offset that is then excluded when the full parametrization is determined. This offset 
is obviously the constant electron mean $d E / d x$ response mentioned above.

There is a strong dependence of UNQCTC on NCTC. As NCTC decreases below $\sim 20$, the mean UNQCTC increases correspondingly. The NCTC correction will be obtained last, and the other corrections are first obtained using tracks with NCTC $\geq 25$. This requirement is of course removed in the final simultaneous fit for all parameters.

No assumptions about the $d E / d x$ resolution will be made until UNQCTC has been parametrized versus all variables. Consequently, the likelihood function used

in the first fits will not be correctly normalized, and the uncertainties returned by the fits can be ignored. The resolution is, of course, a function of NCTC; fewer samples result in a poorer $d E / d x$ measurement. An estimate of the resolution is obtained after the last fit in each series, and is then used to normalize the likelihood function for a refit.

\section{Conversion Sample Preparation}

A large sample of conversion electrons can be found in the ECLB BPAD sample. This was demonstrated in Section 3.4.1, where trigger electrons from conversions were identified and removed from the primary dataset. They were removed because they were backgrounds to electrons from $b$ decay. Here, they shall be selected, as conversions provide a pure electron sample for the $d E / d x$ correction procedure.

First, trigger electrons are again selected as described in Section 3.4.1. Conversion partner tracks are found using the requirements of Table 3.2, with the following additions and/or changes:

- There must be one and only one partner track which passes the cuts.

- $p_{\mathrm{t}}>0.4 \mathrm{GeV} / \mathrm{c}$

- $\mathrm{NCTC} \geq 10$ 
- Radius of conversion: $22.0<\mathrm{R}_{\text {conv }}<30.0 \mathrm{~cm}$

Approximately 300,000 track pairs meet these requirements. The range of the radius of conversion was picked to select conversions in the material of the VTX-CTC boundary, minimizing background. Tracks with NCTC $<10$ have few unshared hits in the outer CTC superlayers, making the measurement of $d E / d x$ suspect. Only the conversion partners are used to derive the corrections. These tracks are unbiased by the trigger and cover the $p_{\mathrm{t}}$ range of soft electron candidates.

\section{Sectioning Run 1B}

Run $1 \mathrm{~B}$ is divided into three sections for the purposes of correcting $d E / d x$. At the time of run 60900, an accelerator quadrupole focussing magnet was realigned, resulting in very different accelerator operating conditions, especially a much larger instantaneous luminosity. At the time of run 67390, the CTC TDC calibration constants were changed. This had a serious impact on the $d E / d x$ system, and required corresponding alterations in the $d E / d x$ offline software. Consequently, for the corrections, Run 1B is partitioned into runs before 60900, from 60900 to 67390 , and after 67390. Colloquially, these sections are called "LO", "MED", and "HI", respectively.

\section{C.2.1 Path-Length Dependence}

As previously discussed, the largest correction is for path-length. Figure C.1 shows UNQCTC versus $d \ell d r$ for MED conversion electrons with $p_{\mathrm{t}}>3.0 \mathrm{GeV} / \mathrm{c}$. The distribution roughly has the form

$$
\mathrm{UNQCTC}=34.34+41.06 \times\left[1-e^{(1-d l d r)}\right]
$$


as shown by the overlaid curve. The shape does not well describe the mean UNQCTC at small $d \ell d r$. This is due to the sense wire gain saturation at $\theta=90^{\circ}$. A better parametrization, accounting for this effect, is

$$
\mathrm{UNQCTC}=b+Q_{\text {gain }}\left[1-e^{[1-d l d r(1+a|\cos \theta|)(1-|\cos \theta|)]}\right]
$$

Fitting the mean UNQCTC with this functional form gives

\begin{tabular}{lcrr} 
Parameter & LO & MED & \multicolumn{1}{c}{ HI } \\
$b$ & $31.54 \pm 0.061$ & $32.61 \pm 0.029$ & $37.95 \pm 0.045$ \\
$Q_{\text {gain }}$ & $40.71 \pm 0.117$ & $40.43 \pm 0.053$ & $44.20 \pm 0.076$ \\
$a$ & $0.25 \pm 0.008$ & $0.18 \pm 0.004$ & $0.15 \pm 0.005$
\end{tabular}

which clearly shows the differences between the three run ranges. The fitted curves and the mean residual distributions for the LO run range are shown in Figure C.2. Recall that the above uncertainties on the parameters are not correct. The likelihood function is not yet correctly normalized. All fit results shown before Section C.2.4 will also not be correctly normalized.

\section{C.2.2 Luminosity Dependence}

Now that the $d \ell d r$ and the polar angle dependences are handled properly, the effect of the instantaneous luminosity on the mean residuals is examined. The luminosity is correlated with NCTC, but because UNQCTC is independent of NCTC for NCTC $\geq 25$, the shape of the luminosity effect can be examined without bias.

Figure C.3 shows the mean residuals as a function of luminosity for the three run ranges. In each case, the shape is a parabola, with a slope of zero at zero luminosity. A luminosity-squared term, with coefficient Luco, serves as the correction. The superimposed curves in the figure represent a fit for $L u c o$, with the values indicated in the figure caption. 
Equation C.2 is altered to include the luminosity correction

$$
\mathrm{UNQCTC}=b+Q_{\text {gain }}\left[1-e^{[1-d l d r(1+a|\cos \theta|)(1-|\cos \theta|)]}\right]+\text { Luco } \cdot \operatorname{lum}^{2}
$$

and the data from the three run ranges refit, yielding

\begin{tabular}{lrllrllrll} 
Parameter & \multicolumn{2}{c}{ LO } & \multicolumn{4}{c}{ MED } & \multicolumn{2}{c}{ HI } \\
$b$ & 31.63 & \pm & 0.063 & 32.25 & \pm & 0.031 & 38.48 & \pm & 0.047 \\
$Q_{\text {gain }}$ & 40.73 & \pm & 0.117 & 40.47 & \pm & 0.053 & 44.28 & \pm & 0.076 \\
$a$ & 0.25 & \pm & 0.008 & 0.18 & \pm & 0.004 & 0.15 & \pm & 0.005 \\
Luco & -0.0142 & \pm & 0.0019 & -0.0117 & \pm & 0.0002 & -0.0058 & \pm & 0.0001
\end{tabular}

\section{C.2.3 NCTC Dependence}

The NCTC $\geq 25$ requirement is removed to examine the variation of the mean residuals versus NCTC; Figure C.4 shows the distributions. The overlaid curves have the form

$$
\overline{\text { residuals }}(\mathrm{NCTC})=F \cdot\left(e^{-N_{p h i}\left(\mathrm{NCTC}-N_{\text {offset }}\right)}-1\right)
$$

The results of a binned fit for the coefficients are indicated in the figure caption.

Convergence problems arise if a term of this form is added to the parametrization of UNQCTC in Equation C.3, as it is not independent of the coefficient $b$ in that equation. Equation C.4 is obviously equivalent to

$$
\overline{\text { residuals }}(\mathrm{NCTC})=F \cdot e^{-N_{p h i} \mathrm{NCTC}} \cdot e^{N_{p h i} N_{\text {offset }}}-F
$$

The coefficient $b$ already accounts for a constant offset. The introduction of a $-F$ term will induce unpleasant correlations between the offset and the NCTCdependent term. It is appropriate to use $N_{\text {offset }}$ to control the overall scale of the NCTC correction, and use $b$ to adjust the offset. Therefore, $F$ is removed from 
Equation C.4. The complete functional parameterization is

$$
\begin{aligned}
\mathrm{UNQCTC}= & b+Q_{\text {gain }}\left[1-e^{[1-d l d r(1+a|\cos \theta|)(1-|\cos \theta|)]}\right]+ \\
& \text { Luco } \cdot \operatorname{lum}^{2}+\left[e^{-N_{\text {phi }}\left(\mathrm{NCTC}-N_{\text {offset }}\right)}-1\right]
\end{aligned}
$$

\begin{tabular}{|c|c|c|c|c|c|c|}
\hline Parameter & & $\mathrm{O}$ & & IED & & HI \\
\hline$b$ & 32.13 & \pm 0.06 & 33.40 & \pm 0.03 & 38.31 & $\pm \quad 0.07$ \\
\hline$Q_{\text {gain }}$ & 41.41 & \pm 0.08 & 40.81 & \pm 0.03 & 44.58 & 0.04 \\
\hline$a$ & 0.233 & 0.005 & 0.188 & \pm 0.002 & 0.160 & 0.003 \\
\hline Luco & -0.0138 & 0.0013 & -0.0115 & \pm 0.0001 & -0.0068 & 0.0001 \\
\hline$N_{p h i}$ & 0.161 & 0.005 & 0.120 & \pm 0.002 & 0.090 & 0.003 \\
\hline$N_{\text {offset }}$ & 21.61 & 0.304 & 23.76 & \pm 0.25 & 27.66 & \pm 0.60 \\
\hline
\end{tabular}

A simultaneous unbinned fit of all the parameters to the data yields

\section{C.2.4 First Resolution Estimates}

The resolution, a function of NCTC, is measured from the width of the residual distributions in bins of NCTC. The distribution in each bin is fitted with a Gaussian, and the width of each Gaussian as a function of NCTC is parametrized by the function $\frac{A}{\text { NCTC }}$. Figure C.6, from the MED run range, shows the fitted widths versus NCTC along with an estimate of $A$ from a binned fit. A better determination of $A$ is from an unbinned maximum-likelihood fit, yielding

$$
\begin{array}{cccc} 
& \text { LO } & \text { MED } & \text { HI } \\
A & 79.37 \pm 0.63 & 82.14 \pm 0.26 & 94.82 \pm 0.40
\end{array}
$$

\begin{tabular}{|c|c|c|c|c|c|c|}
\hline Parameter & & $\mathrm{O}$ & & IED & & HI \\
\hline$b$ & 32.28 & \pm 0.18 & 33.57 & \pm 0.11 & 38.62 & \pm 0.07 \\
\hline$Q_{\text {gain }}$ & 41.12 & \pm 0.27 & 40.70 & \pm 0.11 & 44.56 & 0.18 \\
\hline$a$ & 0.237 & \pm 0.017 & 0.185 & \pm 0.008 & 0.159 & 0.011 \\
\hline Luco & -0.0149 & \pm 0.0044 & -0.0115 & \pm 0.0003 & -0.0067 & 0.0003 \\
\hline$N_{p h i}$ & 0.179 & $\pm \quad 0.024$ & 0.132 & $\pm \quad 0.010$ & 0.108 & 0.016 \\
\hline$N_{\text {offset }}$ & 20.79 & 0.913 & 22.66 & \pm 0.79 & 24.63 & \pm 2.05 \\
\hline
\end{tabular}

These parametrizations of the resolution are used to normalize the likelihood function for a refit of the six parameters, yielding 


\section{C.3 Aspect Angle Corrections}

The previous fits demonstrate that UNQCTC depends on a number of parameters, most of them geometrical. Actually, each of the parameters depend in turn on the tracks' aspect angle. This effect was masked in the fits by requiring the conversion electrons to have $p_{\mathrm{t}} \geq 3.0 \mathrm{GeV} / \mathrm{c}$, which roughly corresponds to $|a s p c|<0.08$; for tracks down to $0.4 \mathrm{GeV} / \mathrm{c},|a s p c|$ can be as large as 0.6 . In addition, due to the CTC geometry, the $d E / d x$ response of positive tracks does not a priori have to be the same as that for negative tracks. Fitting $b$ as a function of $a s p c$ will constrain the two to be the same.

To study the aspc dependence, the NCTC $\geq 25$ requirement is reimposed and the $p_{\mathrm{t}}$ cut is lowered to $p_{\mathrm{t}} \geq 0.4 \mathrm{GeV} / \mathrm{c}$. The data is divided into bins of $a s p c$, and is fit for the four parameters of Equation C.3 in each bin. The shapes of $b, Q_{\text {gain }}$, $a$, and $L u c o$ versus $a s p c$ are fit to polynomials with terms $a s p c^{n}$ and $|a s p c| a s p c^{m}$. These polynomials then replace the four parameters in the likelihood function (each polynomial coefficient becoming a free parameter).

The NCTC requirement is again relaxed to NCTC $\geq 10$. Equation C.6 is used to fit all six parameters in bins of $a s p c$, allowing the determination of the appropriate expansions for $N_{p h i}$ and $N_{\text {offset }}$. A simultaneous fit to all polynomial coefficients fails to converge when $N_{\text {offset }}$ is replaced by an expansion in this manner. Accordingly, $N_{\text {offset }}$ is maintained as a single parameter, independent of aspc. This does not compromise the quality of the fit, owing to the correlations between $N_{\text {offset }}$ and the other coefficients. The results of the simultaneous fit, for the expansions of the other five parameters, are shown in Figures C.7-C.10. The polynomial expansion for each parameter is listed in Section C.5. 


\section{C.3.1 Final Resolution Determination}

With the full fits completed, the resolutions and their dependence on NCTC must be redetermined. The overall uncertaintiess are only slightly improved. This is not unexpected, given the size of most of the corrections relative to the natural detector resolution. Again, the data is binned in NCTC and a Gaussian is fit to each bin's residual distribution. Figures C.11-C.13 show the fitted widths versus NCTC, along with a binned fit to the function $\frac{A}{\text { NCTC }}+B$. An unbinned likelihood fit for the constants $A$ and $B$ yields

$$
\begin{aligned}
& \text { LO MED HI }
\end{aligned}
$$

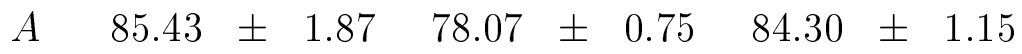

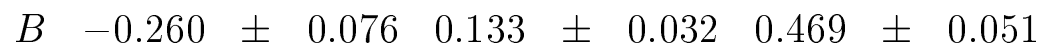

\section{C.4 The Universal Curve}

Given the completed set of corrections, they can be applied to other particle types and are used to prepare the mean $d E / d x$ prediction curves versus $\beta \gamma$. The parametrization of the conversion electron mean UNQCTC just derived included the term

$$
b=b_{00}+\sum_{n=1}^{N} b_{n} \cdot a s p c^{n}+|a s p c| \sum_{m=1}^{M} c_{m} \cdot a s p c^{m}
$$

The powers of aspc remove the $d E / d x$ aspect angle dependence; the offset $b_{00}$ is the mean electron $d E / d x$ response itself (recall that the $d E / d x$ of electrons is constant over the entire momentum range of interest). The corrected QCTC is therefore UNQCTC minus the full parametrization, omitting $b_{00}$ from the latter.

The derivation of the predicted $d E / d x$ response with momentum requires clean samples of other particles in addition to the conversion electron sample used to derive the corrections. The ECLB dataset is used to search for pions from $K_{S}^{0} \rightarrow \pi \pi$ and protons from $\Lambda^{0} \rightarrow \mathrm{p} \pi$, and the PSIB dataset for muons from $J / \psi \rightarrow \mu \mu$. Each 
of these particles were selected with standard selection criteria which will not be described here. The invariant mass distributions for the three particle candidates are shown in Figures C.14-C.16.

As the two-track particle candidates are not particularly free of background, signal and sideband regions are defined under and around the particle mass peaks. The sideband $d E / d x$ response can then be subtracted from the signal $d E / d x$ response when determining the mean corrected QCTC versus $\beta \gamma$. The regions are shown as the shaded areas in Figures C.14-C.16. The subtraction is simply performed by evaluating for each bin in $\beta \gamma$

$$
\frac{\sum_{\text {signal }} \mathrm{QCTC}-\sum_{\mathrm{bkg}} \mathrm{QCTC}}{N_{\mathrm{sig}}-N_{\mathrm{bkg}}}
$$

with the appropriately propagated uncertainties.

The proton sample from $\Lambda^{0}$ decay can be supplemented by searching for particles consistent with having been knocked out of the beampipe. Tracks with impact parameter significances greater than 100 are good candidates. For momentum between 0.5 and $0.9 \mathrm{GeV} / \mathrm{c}$, protons are sufficiently separated from pions, kaons, and deuterons that a simple window cut on QCTC is enough to select protons without much bias. Figure C.17 shows the distributions and the defined signal regions for the MED run range; those for the LO and HI ranges are fundamentally similar.

\section{C.4.1 Fitting the Curve}

The $d E / d x$ prediction curve has the functional form

$$
\mathrm{QCTC}=\frac{c_{0}+c_{1} \log \left[\frac{\beta \gamma}{\beta \gamma+b}\right]}{\beta^{2}}+a_{1}(\beta-1)+a_{2}(\beta-1)^{2}
$$


giving five parameters $\left(c_{0}, c_{1}, b, a_{1}\right.$, and $\left.a_{2}\right)$ in total. The general features of the curve are easily understood. At low $\beta \gamma$, the curve exhibits a $1 / \beta^{2}$ falloff, resulting from the fact that a faster-moving particle spends less time near a given atom in the CTC gas and hence is less likely to transfer energy to the atom. After passing through a minimum, $d E / d x$ rises logarithmically. The "relativistic rise" is a result of the relativistic increase in the incident particle's transverse electric field. The eventual flattening of the $d E / d x$ curve (the "plateau region") is due to polarization of the CTC gas.

Figures C.18-C.20 show the variation of the mean corrected QCTC with $\beta \gamma$. All particle types were used to prepare the figures, with sideband subtraction where appropriate. Superimposed on each figure is a fit of Equation C.9 to the data. The fitted values of the parameters are also given in the figures. 


\section{C.5 Polynomial Expansions from the Final Fit}

\section{LO run range}

$$
\begin{aligned}
b= & 31.13-107.6 \cdot a s p c^{2}-46.48 \cdot a s p c^{3}-155.9 \cdot a s p c^{4} \\
& +14.84 \cdot|a s p c|+31.47 \cdot|a s p c| a s p c+21.00 \cdot|a s p c| a s p c^{2} \\
Q_{\text {gain }}= & 40.93-17.48 \cdot a s p c+70.48 \cdot a s p c^{2} \\
a= & 0.2428 \\
\text { Luco }= & -0.015+0.102 \cdot a s p c-0.055 \cdot a s p c^{2}-0.581 \cdot a s p c^{3} \\
N_{p h i}= & 0.0862-0.0602 \cdot a s p c-1.289 \cdot a s p c^{2} \\
& +0.362 \cdot|a s p c|+0.410 \cdot|a s p c| a s p c \\
N_{\text {offset }}= & 26.06
\end{aligned}
$$

\section{MED run range}

$$
\begin{aligned}
b= & 31.98-290.8 \cdot a s p c^{2}-41.29 \cdot a s p c^{3}-675.4 \cdot a s p c^{4} \\
& +36.75 \cdot|a s p c|+32.62 \cdot|a s p c| a s p c+735.0 \cdot|a s p c| a s p c^{2} \\
Q_{\text {gain }}= & 40.33-15.74 \cdot a s p c+72.13 \cdot a s p c^{2} \\
a= & 0.2636+21.03 \cdot a s p c^{2}+59.80 \cdot a s p c^{4}-2.455 \cdot|a s p c| \\
& -62.02 \cdot|a s p c| a s p c^{3} \\
\text { Luco }= & -0.0109+0.0134 \cdot a s p c+0.0163 \cdot a s p c^{2} \\
& -0.0152 \cdot|a s p c|-0.0357 \cdot|a s p c| a s p c^{2} \\
N_{p h i}= & 0.0987-0.0133 \cdot a s p c-0.152 \cdot a s p c^{2} \\
N_{\text {offset }}= & 26.68
\end{aligned}
$$

\section{HI run range}

$$
\begin{aligned}
b= & 38.66-5.470 \cdot a s p c-35.28 \cdot a s p c^{2}+4.138 \cdot|a s p c|+2.862 \cdot|a s p c| a s p c \\
Q_{\text {gain }}= & 44.33-18.70 \cdot a s p c+82.85 \cdot a s p c^{2} \\
a= & 0.1463+0.1260 \cdot a s p c+0.0912 \cdot|a s p c|+0.0711 \cdot|a s p c| a s p c \\
\text { Luco }= & -0.0053+0.0025 \cdot a s p c+0.1525 \cdot a s p c^{2}+0.0424 \cdot a s p c^{3} \\
& -0.0406 \cdot|a s p c|-0.2165 \cdot|a s p c| a s p c^{2} \\
N_{p h i}= & 0.0839-7.623 \cdot a s p c^{2}+0.2095 \cdot a s p c^{3}-17.34 \cdot a s p c^{4} \\
& +1.031 \cdot|a s p c|+19.70 \cdot|a s p c| a s p c^{2} \\
N_{\text {offset }}= & 24.10
\end{aligned}
$$




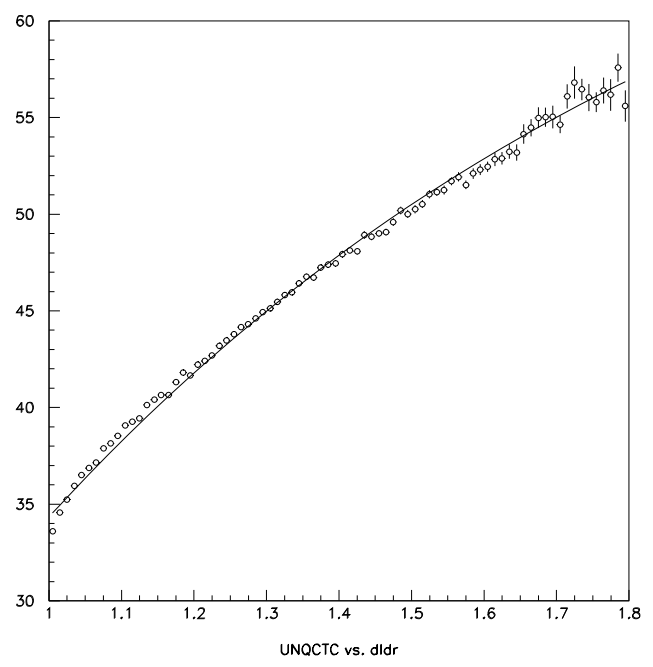

Figure C.1: UNQCTC versus path-length for electrons with $p_{\mathrm{t}}>3.0 \mathrm{GeV} / \mathrm{c}$ in the MED run range. The overlaid curve is $34.34+41.06 *\left[1-e^{(1-d \ell d r)}\right]$.
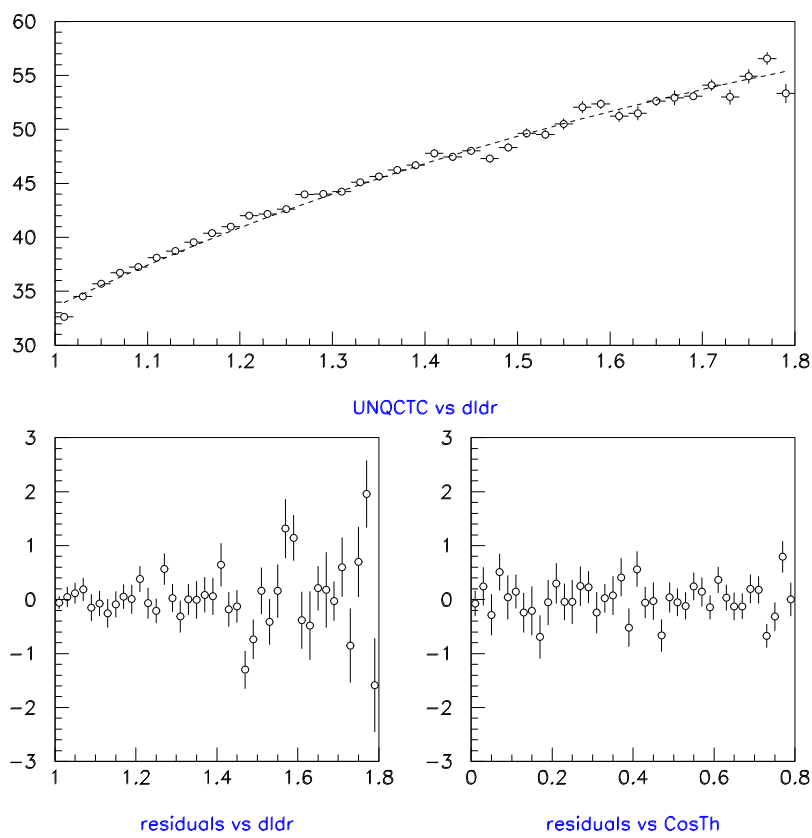

Figure C.2: Top: UNQCTC versus path-length for LO runs, with the fit superimposed. Electrons are required to have $p_{\mathrm{t}}>3.0 \mathrm{GeV} / \mathrm{c}$, and NCTC $\geq 25$.

Bottom left: Mean residuals versus path-length.

Bottom right: Mean residuals versus $|\cos \theta|$. 


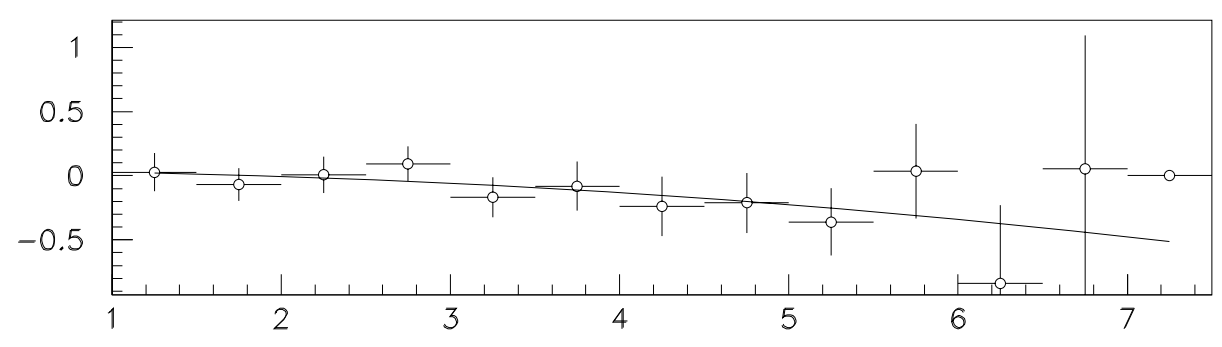

mean residuals $v$ s. Iuminosity $(10 * * 30)-$ LO runs

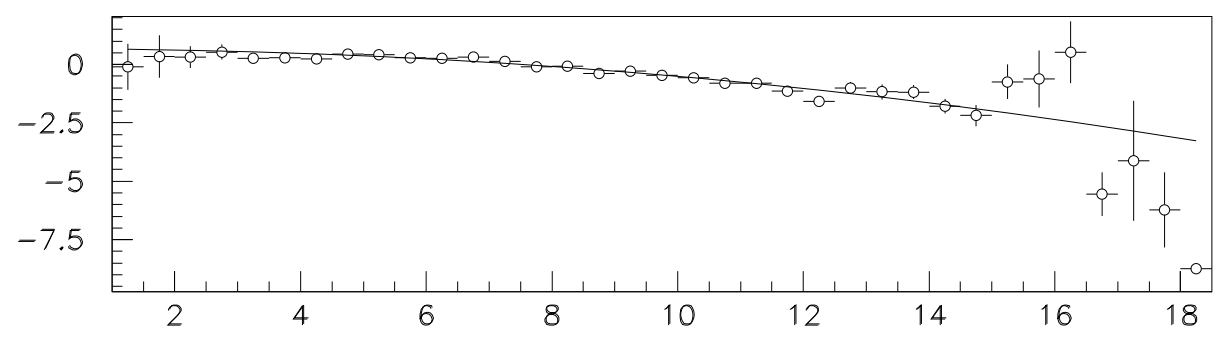

mean residuals vs. Iuminosity $(10 * * 30)$ - MED runs

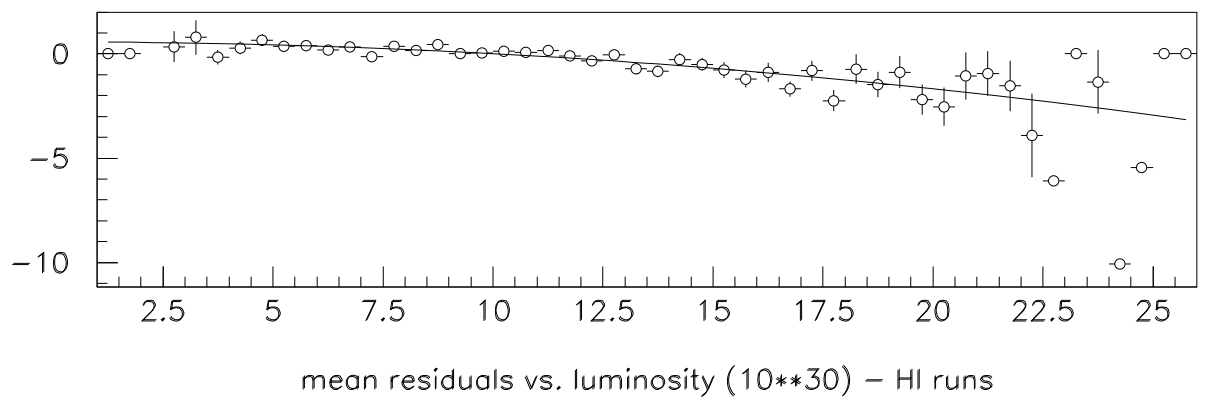

Figure C.3: Mean residuals versus instantaneous luminosity for LO, MED, and HI runs, parametrizing the shape with the parabola $L u c o \cdot \mathrm{lum}^{2}$. The fits yield

$\begin{array}{ccc}\text { LO } & \text { MED } & \text { HI } \\ -0.0104 & -0.0118 & -0.0056\end{array}$



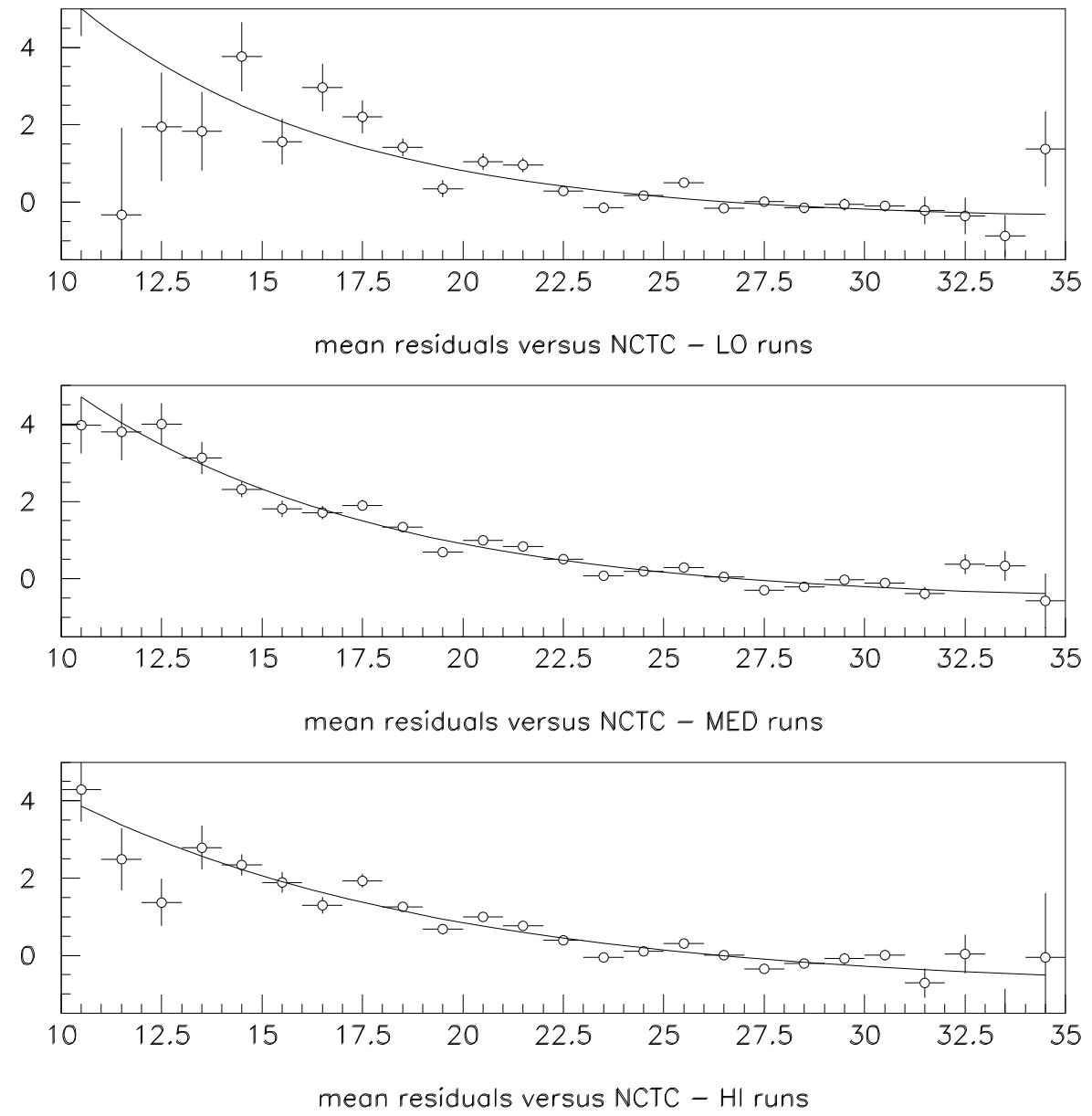

Figure C.4: Mean residuals versus NCTC for LO, MED, and HI runs. The overlaid curves have the form $F\left(e^{-N_{p h i}\left(\mathrm{NCTC}-N_{\text {offset }}\right)}-1\right)$; the fits yield

$\begin{array}{lrrr} & \text { LO } & \text { MED } & \text { HI } \\ F & 0.459 & 0.611 & 0.878 \\ N_{\text {phi }} & 0.154 & 0.132 & 0.106 \\ N_{\text {offset }} & 26.617 & 26.864 & 26.376\end{array}$



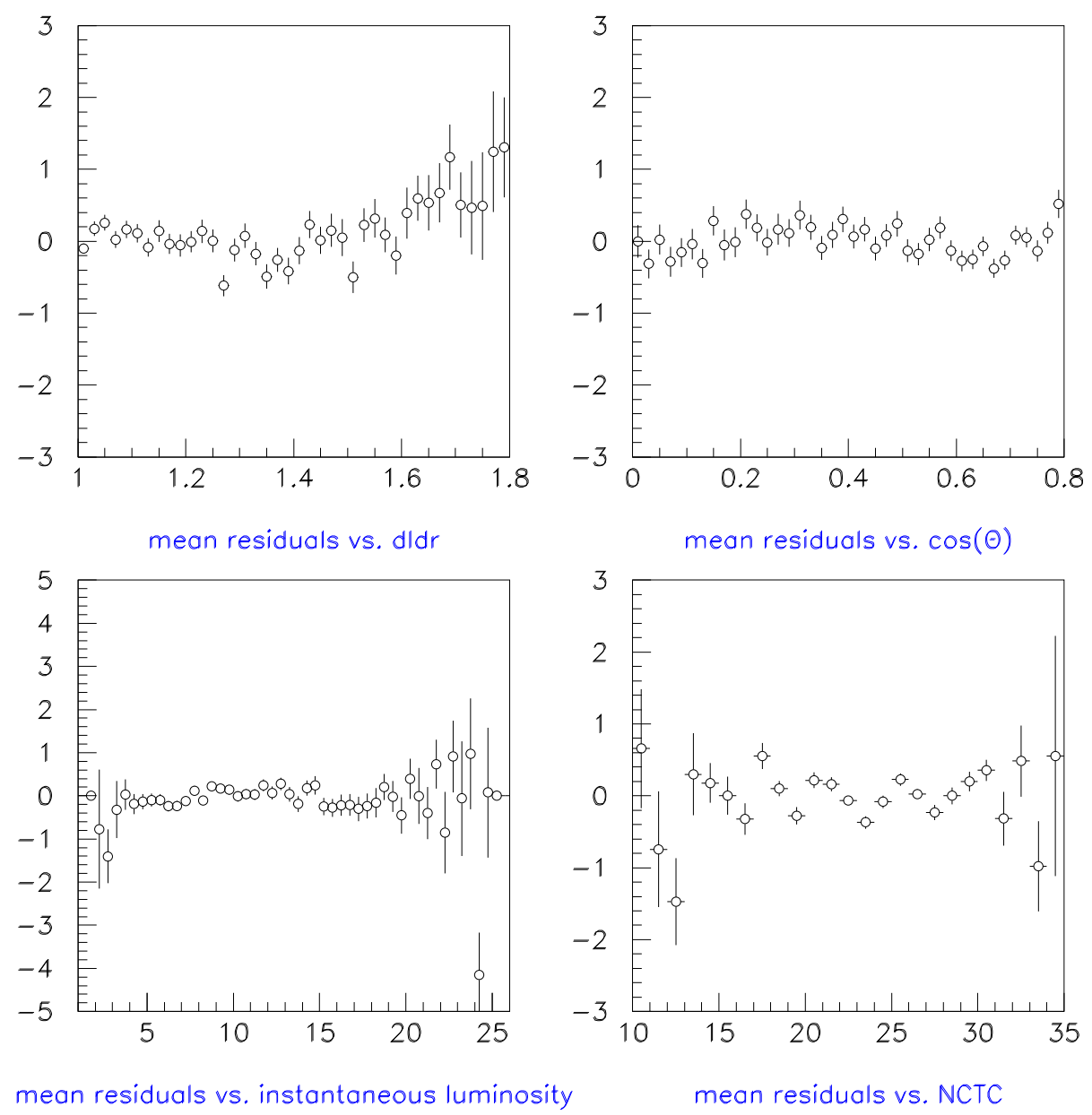

Figure C.5: Mean residuals versus $d \ell d r,|\cos \theta|$, instantaneous luminosity, and NCTC for conversion electrons with $p_{\mathrm{t}}>3.0 \mathrm{GeV} / \mathrm{c}$ in HI runs. 


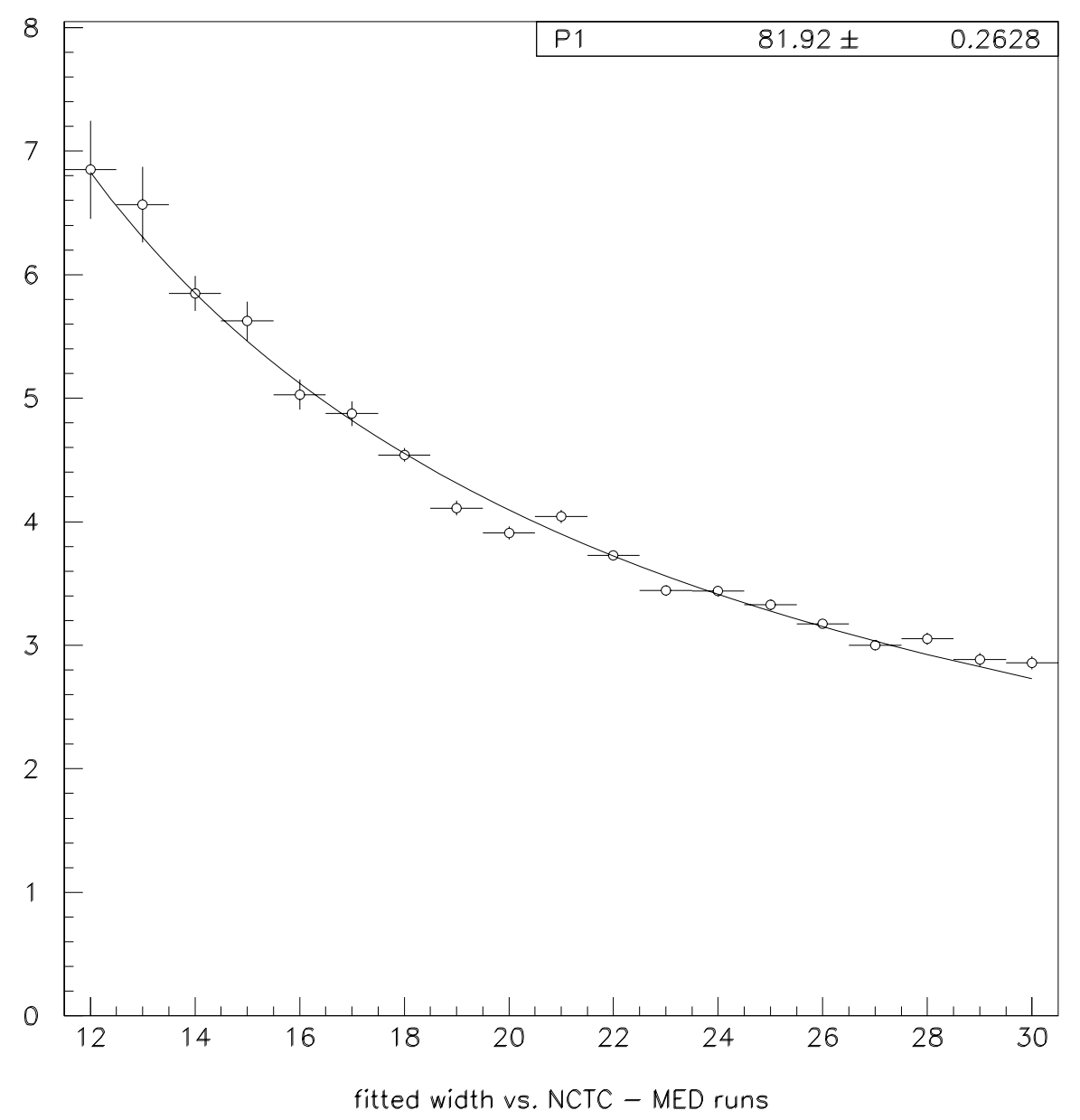

Figure C.6: Fitted width versus NCTC for MED runs. The superimposed curve is $\frac{A}{\text { NCTC }}$ The result of a binned fit for $A$ is indicated in the upper right corner. 

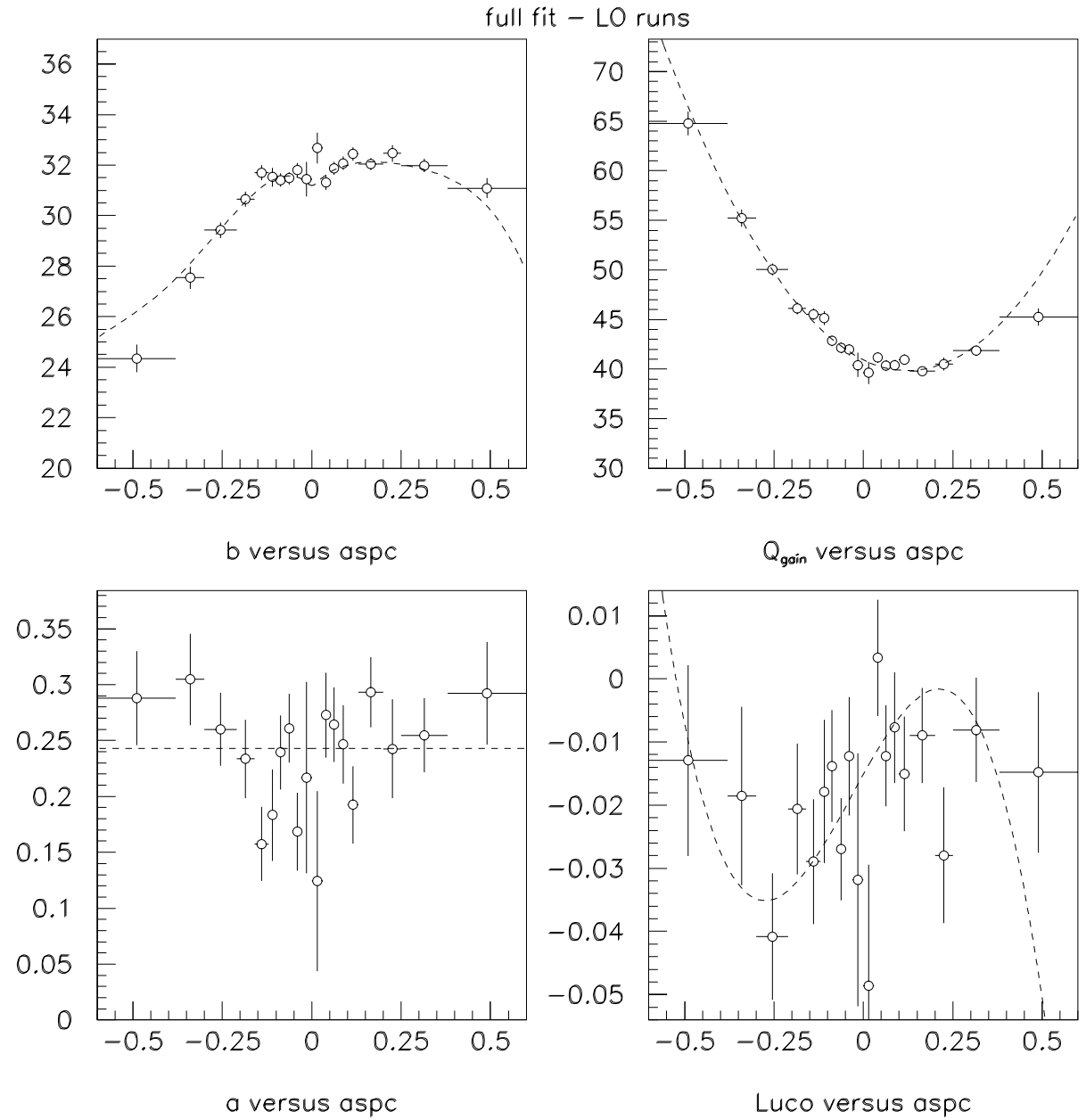

Figure C.7: $b, Q_{\text {gain }}, a$, and Luco versus aspc for LO runs, NCTC $\geq 10$, and $N_{\text {offset }}$ fixed. The superimposed curves are the results of the maximum likelihood fit. 

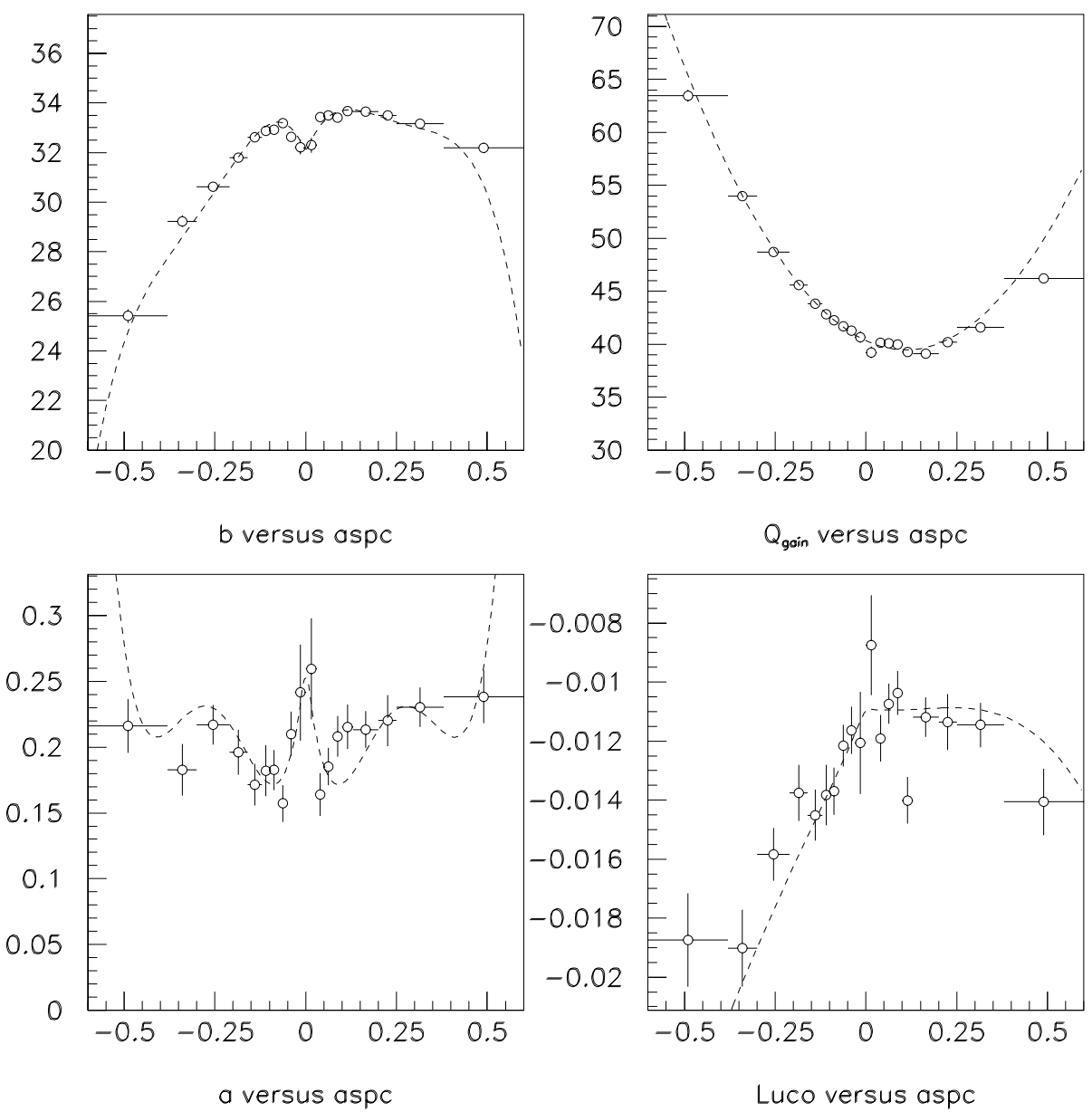

Figure C.8: $b, Q_{\text {gain }}, a$, and Luco versus aspc for MED runs, NCTC $\geq 10$, and $N_{\text {offset }}$ fixed. The superimposed curves are the results of the maximum likelihood fit. 

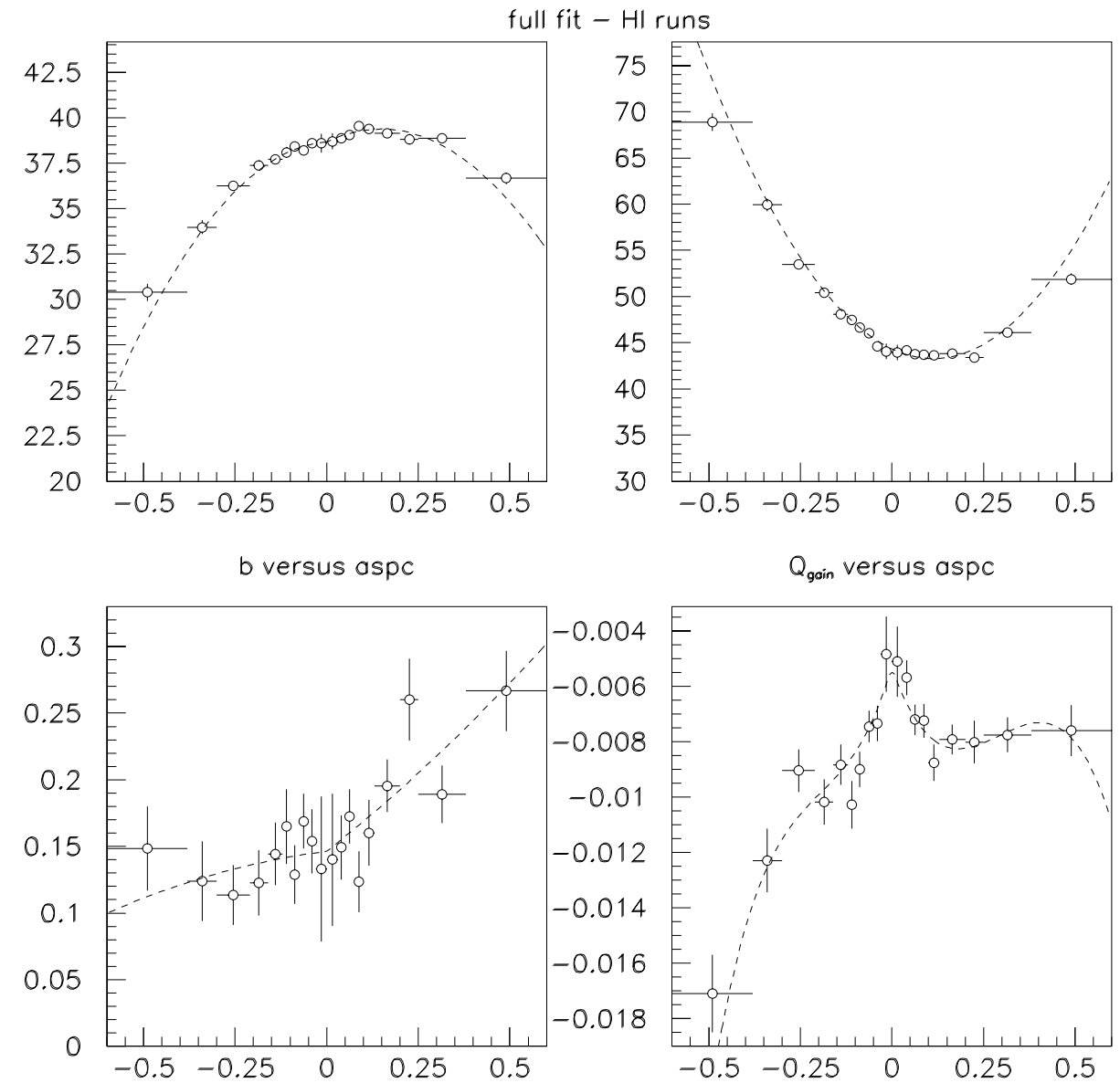

$Q_{\text {goin }}$ versus aspc

a versus aspc

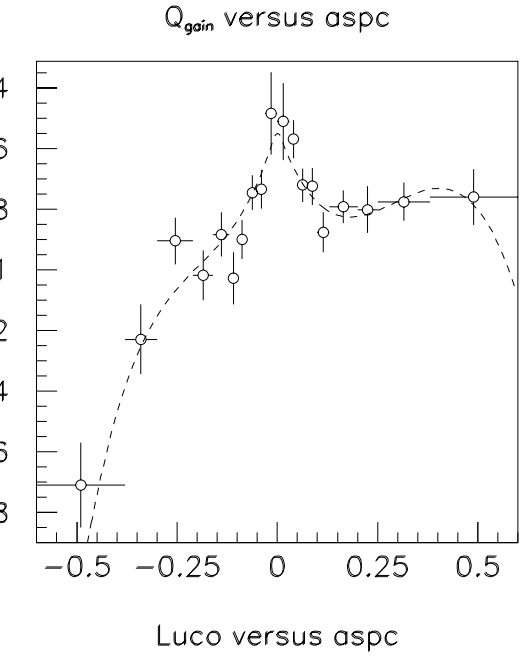

Figure C.9: $b, Q_{\text {gain }}, a$, and Luco versus aspc for HI runs, NCTC $\geq 10$, and $N_{\text {offset }}$ fixed. The superimposed curves are the results of the maximum likelihood fit. 


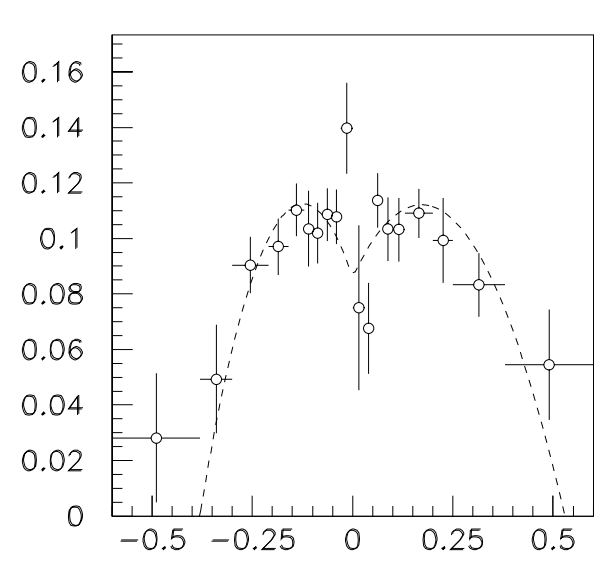

$N_{\text {ohi }}$ versus aspc

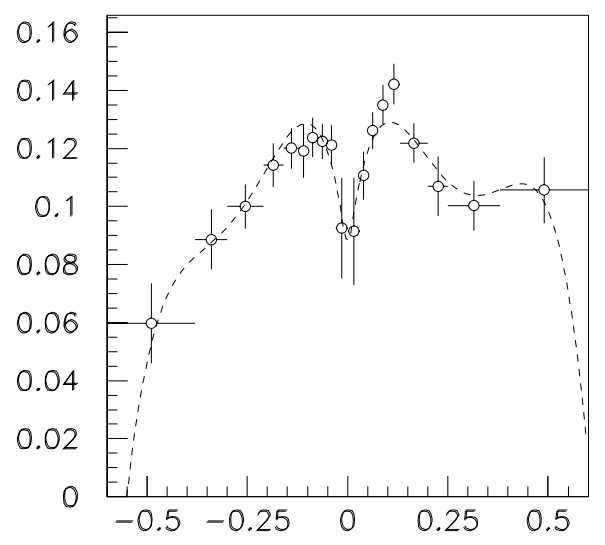

$N_{\text {phi }}$ versus aspc

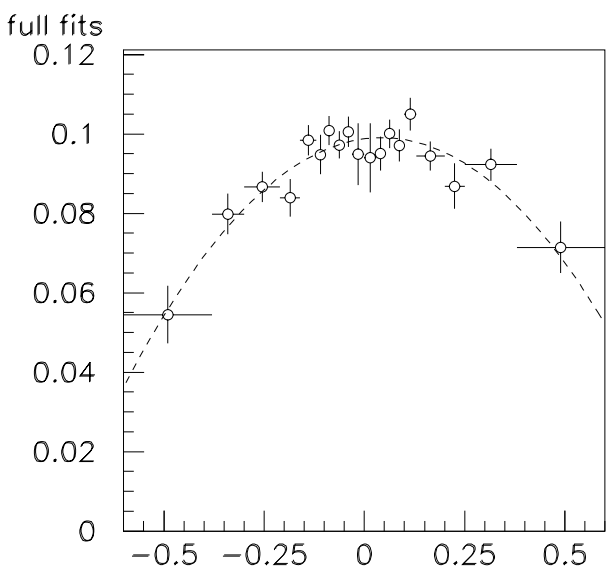

$N_{\text {phi }}$ versus aspc

Figure C.10: $N_{p h i}$ versus aspc for NCTC $\geq 10$ and $N_{\text {offset }}$ fixed, for LO (upper left), MED (upper right) and HI (lower left) runs. The superimposed curves are the results of the maximum likelihood fit. 

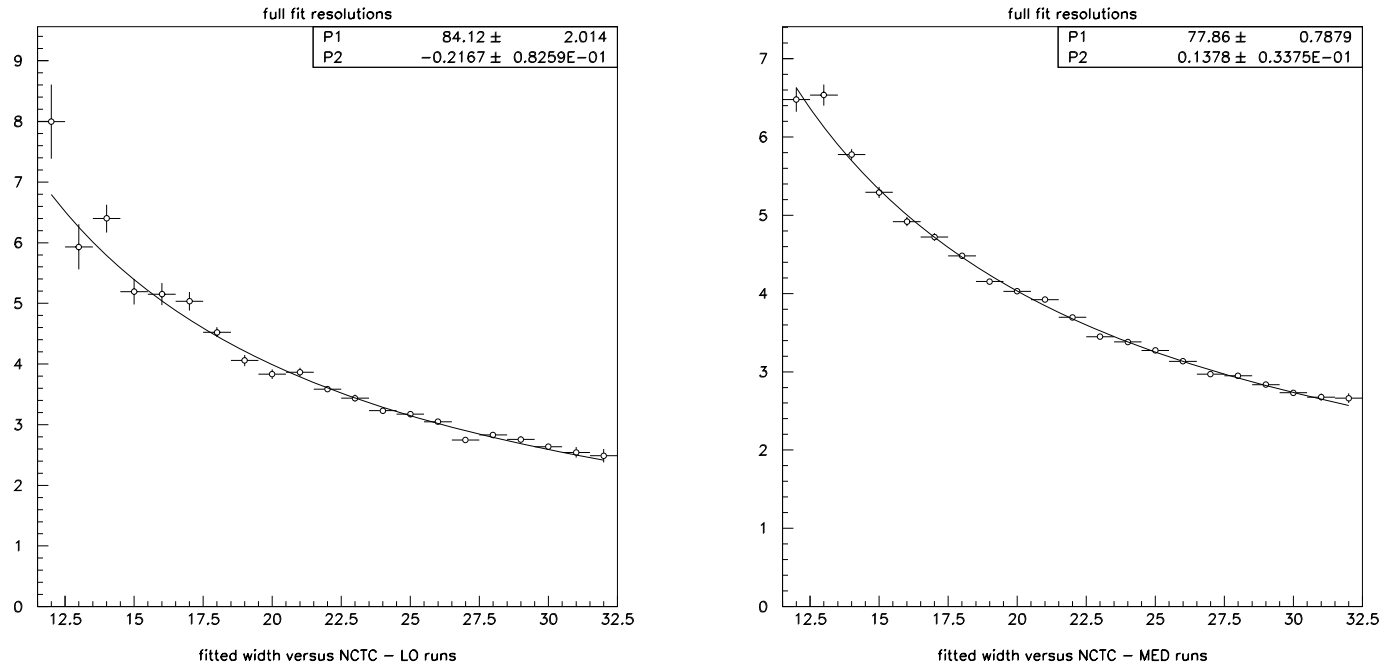

Figure C.11: Fitted width versus NCTC Figure C.12: Fitted width versus NCTC for the full fit in LO runs. The superimposed for the full fit in MED runs. The superimcurve is a binned fit to $\frac{\mathrm{A}}{\mathrm{NCTC}}+B$. $A$ and $B$ posed curve is a binned fit to $\frac{\mathrm{A}}{\mathrm{NCTC}}+B$. $A$ are indicated in the upper right corner (in and $B$ are indicated in the upper right corner that order). (in that order).

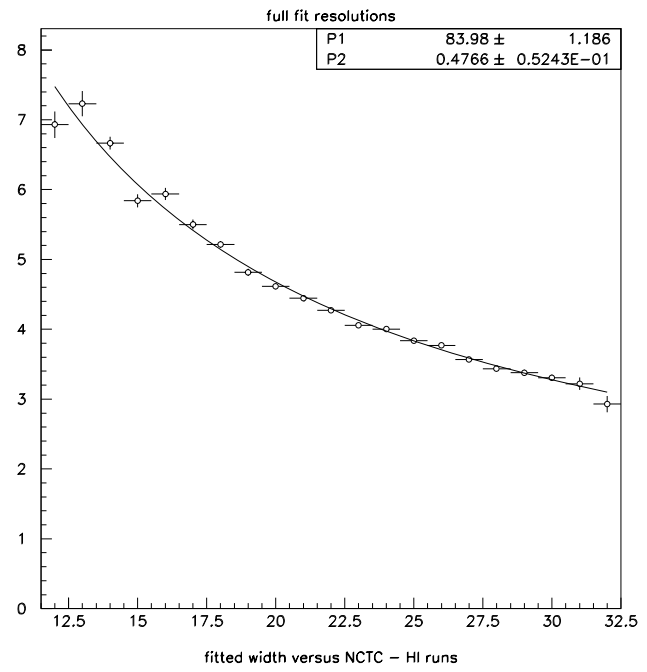

Figure C.13: Fitted width versus NCTC for the full fit in HI runs. The superimposed curve is a binned fit to $\frac{\mathrm{A}}{\mathrm{NCTC}}+B . A$ and $B$ are indicated in the upper right corner (in that order). 

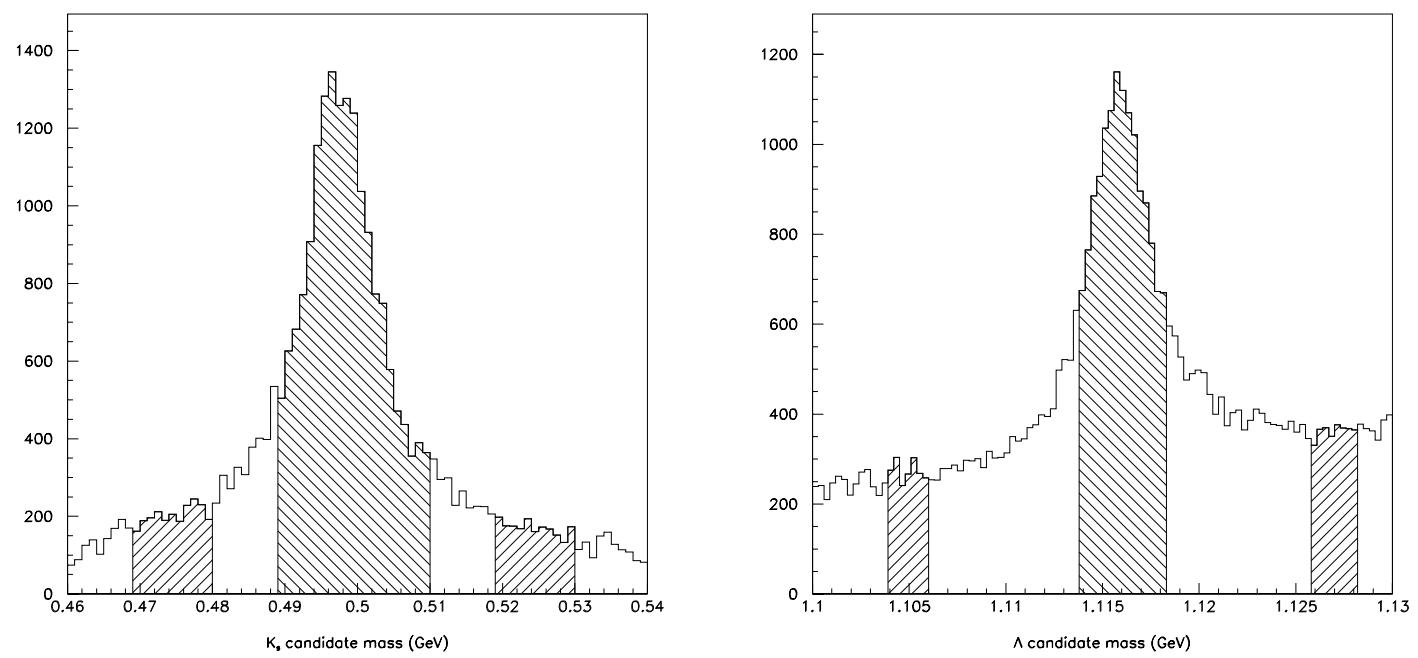

Figure C.14: The invariant mass distrib- Figure C.15: The invariant mass distribution of $K_{s}$ candidates. The signal region ution of $\Lambda^{0}$ candidates. The signal region and the sideband regions are delimited by and the sideband regions are delimited by the shaded areas. the shaded areas.
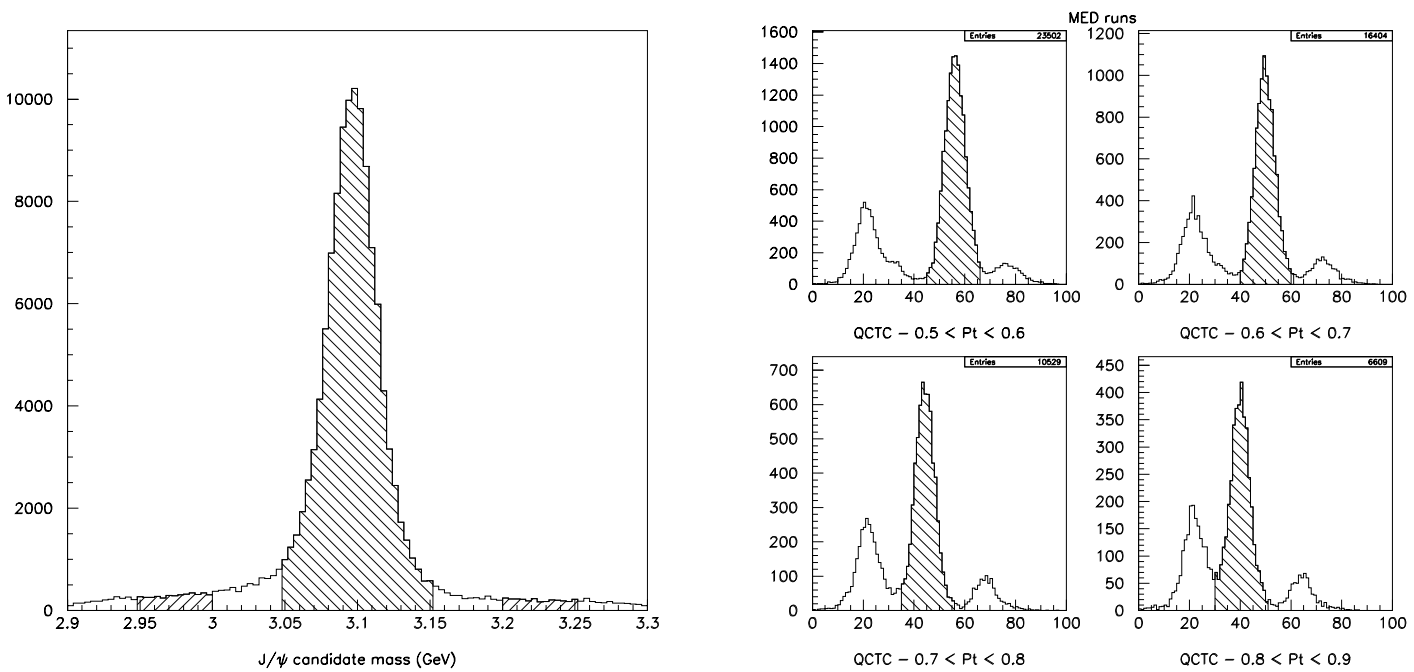

Figure C.16: The invariant mass distrib- Figure C.17: QCTC of tracks with ution of $\mathrm{J} / \psi$ candidates. The signal region $\left|D / \sigma_{D}\right|>100$ in MED runs, in bins of moand the sideband regions are delimited by the mentum. The signal region is delimited by shaded areas. the shaded area. 

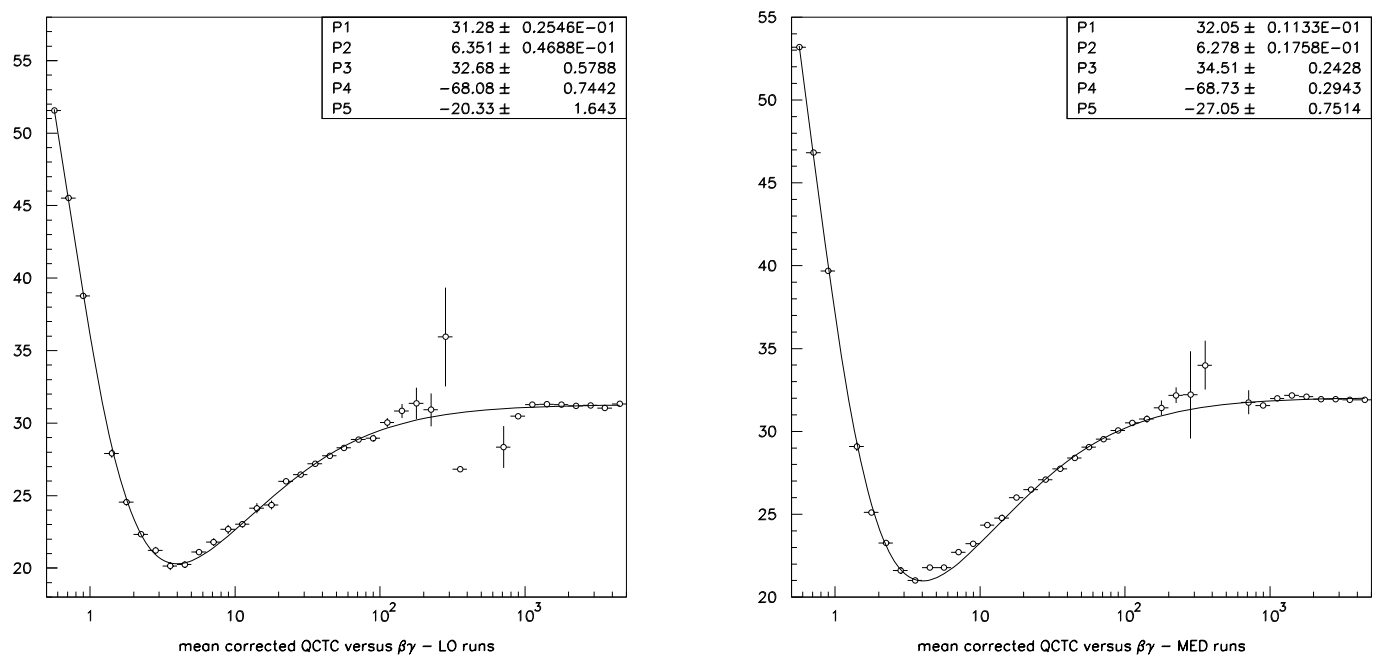

Figure C.18: The mean corrected QCTC Figure C.19: The mean corrected QCTC versus $\beta \gamma$ for all particle types in LO runs. versus $\beta \gamma$ for all particle types in MED runs.

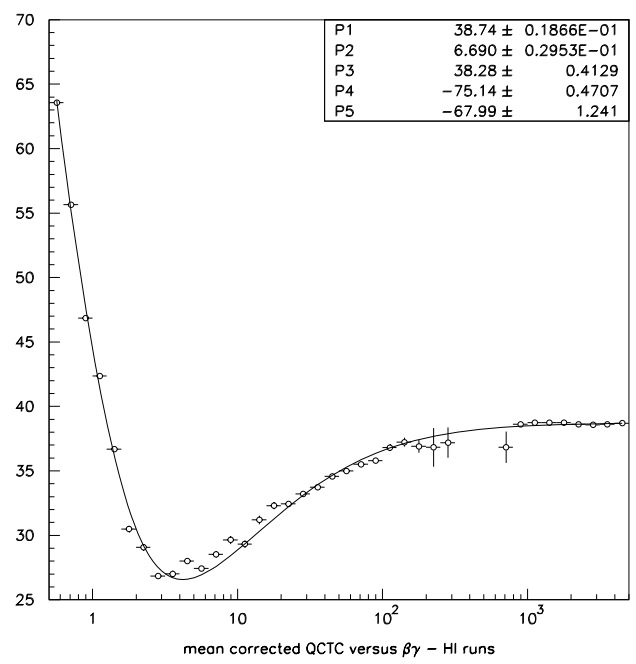

Figure C.20: The mean corrected QCTC versus $\beta \gamma$ for all particle types in HI runs.

The superimposed curves in Figures C.18-C.20 are the result of parametrizing the data with Equation C.9. The fitted values of the five parameters are indicated on each plot (in order, $c_{0}, c_{1}, b, a_{1}$, and $a_{2}$ ). 